\title{
Genotype - phenotype relationship in Drosophila type IV collagen mutants
}

\author{
Ph.D. THESIS
}

András Attila Kiss

\author{
Department of Medical Biology \\ Doctoral School of Multidisciplinary Medicine \\ Faculty of Medicine \\ University of Szeged
}

Supervisors: Dr. Mátyás Mink and Prof. Dr. Zsolt Boldogkői

Szeged

2020 



\section{Publications directly related to the subject of thesis}

I. Drosophila basement membrane collagen col4a1 mutations cause severe myopathy

Kelemen-Valkony I ${ }^{\mathrm{a}}$, Kiss $\mathrm{M}^{\mathrm{a}}$, Csiha $\mathrm{J}^{\mathrm{a}}$, Kiss $\mathbf{A}^{\mathrm{a}}$, Bircher $\mathrm{U}^{\mathrm{a}}$, Szidonya $\mathrm{J}^{\mathrm{a}}$, Maróy $\mathrm{P}^{\mathrm{a}}$, Juhász $\mathrm{G}^{\mathrm{c}}$, Komonyi $\mathrm{O}^{\mathrm{a}}$, Csiszár $\mathrm{K}^{\mathrm{b}}$, Mink $\mathrm{M}^{\mathrm{a}, *}$

Matrix Biology (2012)

https://doi.org/10.1016/j.matbio.2011.09.004

MTMT azonosító: 2104514

IF: $3.19(\mathbf{Q 2})$

II. Drosophila type IV collagen mutation associates with immune system activation and intestinal dysfunction

Márton Kiss ${ }^{\mathrm{a}}$, András A. Kiss ${ }^{\mathrm{a}}$, Monika Radicsa ${ }^{\mathrm{a}}$, Nikoletta Popovics ${ }^{\mathrm{a}}$, Edit Hermesz ${ }^{\mathrm{b}}$, Katalin Csiszár ${ }^{\mathrm{c}}$ and Mátyás Mink ${ }^{\mathrm{a}}$

Matrix Biology (2016)

https://doi.org/10.1016/j.matbio.2015.09.002

MTMT azonosító: 2962945

IF:7.4 (Q1)

III. Altered stress fibers and integrin expression in the Malpighian epithelium of Drosophila type IV collagen mutants

András A. Kiss ${ }^{\mathrm{a}}$, NikolettaPopovicsa ${ }^{\mathrm{a}}$, GáborSzabóa ${ }^{\mathrm{a}}$, Katalin Csiszár ${ }^{\mathrm{b}}$, Mátyás Mink ${ }^{\mathrm{a}}$,* Data in Brief (2016)

https://doi.org/10.1016/j.dib.2016.03.059

MTMT azonosító: 3056130

IF: 0 (Q2)

IV. 4-Hydroxy-2-nonenal Alkylated and Peroxynitrite Nitrated Proteins Localize to the Fused Mitochondria in Malpighian Epithelial Cells of Type IV Collagen Drosophila Mutants

András A. Kiss ${ }^{1}$, Nikoletta Popovics ${ }^{1}$, Zsolt Boldogkői ${ }^{1}$, Katalin Csiszár ${ }^{2}$ and Mátyás Mink $^{1}$

BioMed Research International (2018)

https://doi.org/10.1155/2018/3502401

MTMT azonosító: 3326500

IF: $2.197(Q 2)$

V. Novel Phenotypic Elements of Type IV Collagenopathy Revealed by the Drosophila Model

András A. Kiss ${ }^{1}$, Nikoletta Somlyai-Popovics ${ }^{1}$, Vilmos Tubak ${ }^{2}$, Zsolt Boldogköi ${ }^{1}$, Katalin Csiszár ${ }^{3}$ and Mátyás Mink ${ }^{1 *}$

Applied Sciences (2019) 
https://doi.org/10.3390/app9102083

MTMT azonosító: 30686350

IF: $2.217(\mathbf{Q} 2)$

VI. Type IV Collagen Is Essential for Proper Function of IntegrinMediated Adhesion in Drosophila Muscle Fibers

András A. Kiss ${ }^{1}$, Nikoletta Somlyai-Popovics ${ }^{1}$, Márton Kiss ${ }^{1}$, Zsolt

Boldogkö $i^{1}$, Katalin Csiszár ${ }^{2}$ and Mátyás Mink ${ }^{1}$

International Journal of Molecular Sciences (2019)

https://doi.org/10.3390/ijms20205124

MTMT azonosító: 30881918

IF: $4.183(Q 2)$

\section{Publications indirectly related to the subject of thesis}

VII. Muscle dystrophy is triggered by type IVcollagen alleles affecting integrin binding sites directly or indirectly in Drosophila

Márton Kiss ${ }^{1}$, Ildikó Kelemen-Valkony ${ }^{1}$, András Kiss ${ }^{1}$, Brigitta Kiss ${ }^{2}$, Katalin Csiszár ${ }^{3}$, Mátyás Mink ${ }^{1}$

XXIIIed FECTS and ISMB Joint Meeting, August 25th-29th, 2012, Katowice, Poland http://www.actabp.pl/pdf/Supl1_12/s3.pdf

MTMT azonosító: 3240354

IF: 1.185

Commulative impact factor: 20.372 


\section{List of abbreviations}

AA: Amino acid

ADP: Adenosine diphosphate

AMPs: Anti microbial peptides

ATP: Adenosine triphosphate

BSA: Bovine serum albumin

BM: Basement membrane

Ca: Calcium

CyRoi: Balancer chromosome

DAPI: 4',6-diamidino-2-phenylindole

DTS: Dominant temperature sensitive

ECM: Extracellular matrix

EMS: Ethyl methane sulphonate

ER: Endoplasmic reticule

FITC: Fluorescein isothiocyanate

GBM: Glomerular basement membrane

GFP: Green fluorescent protein

HNE: 4-Hydroxy-2-nonenal
kDa: Kilodalton

MHC: Myosin heavy chain

MLC: Myosin light chain

mRNA: Messenger ribonucleic acid

NCL: Non-collagen like

NO: Nitric oxide

NOS: Nitrogen oxide species

NT: Nitrotyrosine

PBS: Phosphate buffered saline

PFA: Paraformaldehyde

$\mathrm{P}\left\{\mathrm{w}^{*}\right.$ sqh-EYFP-Mito $\}$ : mitochondria labelled mutant

SR: Sarcoplasmic reticule

TBS: Tris-buffered saline

TUNEL: Terminal deoxynucleotidyl transferase dUTP nick end labeling vkg: Viking 


\section{Table of contents}

Publications directly related to the subject of thesis ......................................................... 1

Publications indirectly related to the subject of thesis ........................................................ 2

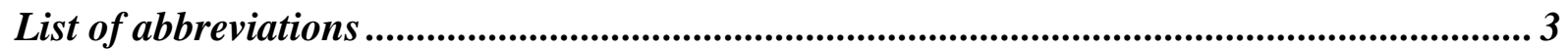

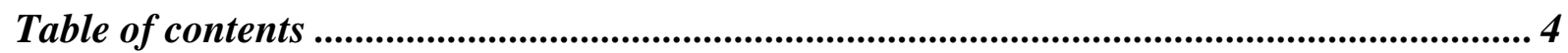

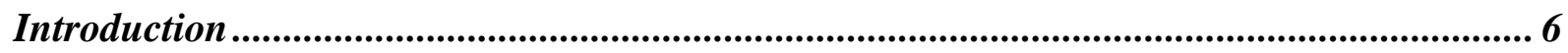

The extracellular space and its role ................................................................................6 6

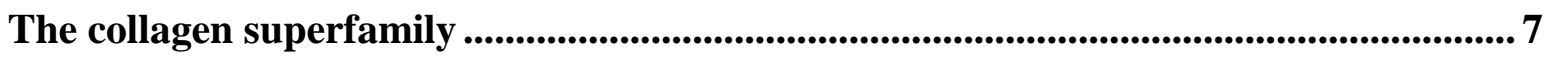

Location of type IV collagen genes in the genome ......................................................8

Biosynthesis, modification and molecular structure of type IV collagen .......................8

The types of network created by type IV collagen ............................................................... 10

Type IV collagen plays a central role in muscle development and structure formation

Diseases associated with type IV collagen mutations in human pathology .................. 11

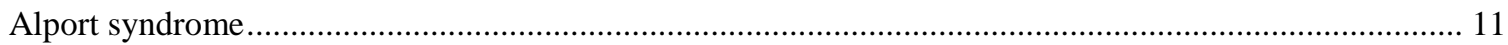

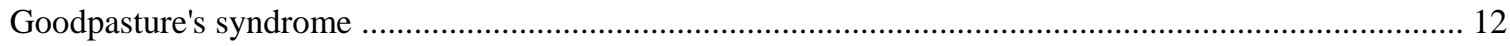

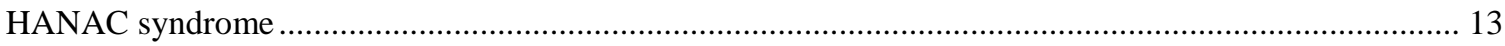

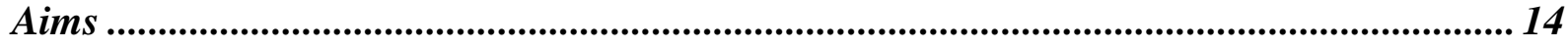

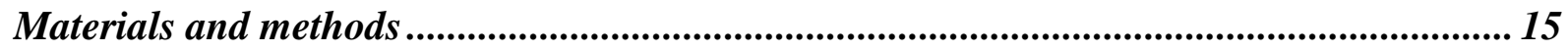

List and maintenance of Drosophila strains................................................................................. 15

The col4a1 mutant strains used in this study (with old and new names) ....................... 15

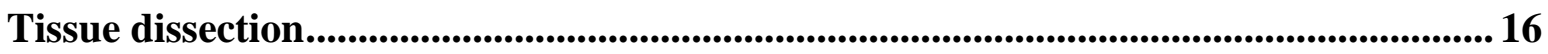

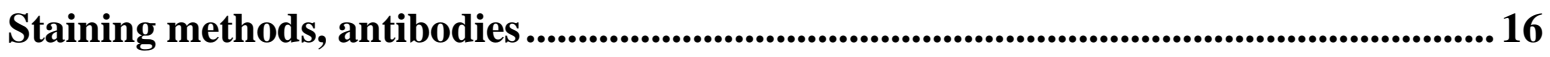

Quantitative fluorescence confocal microscopy ..................................................................... 17

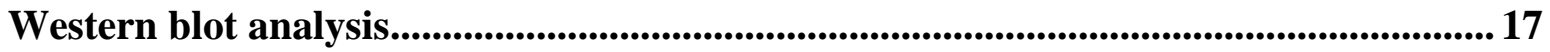

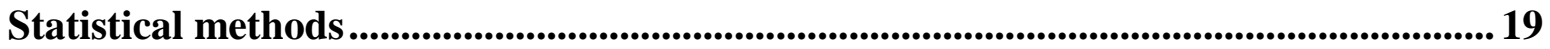

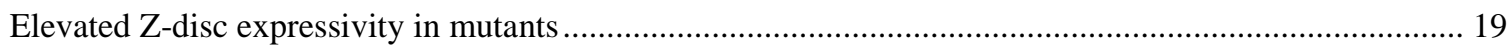

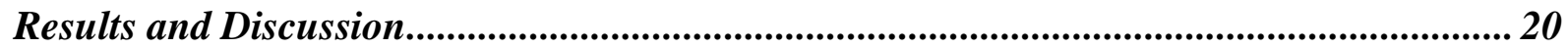

Decreased amount of COL4A1 protein in DTS-L3 mutant at $29^{\circ} \mathrm{C}$............................. 20

Col4a1 mutations cause sarcomeric disorders in common oviduct muscle tissue ........ 22

Z-disc confusion, streaming and improper integrin expression in muscle tissue due to col4a1 mutations 
Myopathic disorders related to col4a1 mutations in the common oviduct of Drosophila melanogaster 28

Structural changes in the basal membrane components of the midgut in col4a1 mutants 30

Disorder of the excretory system of Drosophila melanogaster col4a1 mutants. 34

Changes at the expression level of actin, COL4A1 and integrin in the Malphigian tubules. 35

Elevated peroxynitrite levels and mitochondrial fusion in mutant Malpighian tubules

Elevated levels of alkylated proteins and uneven distribution of mitochondria in the col4a1 mutants Malpighian tubules 41

Lipid peroxidation in the cytoplasmatic membrane of mutant Malpighian tubules ... 42

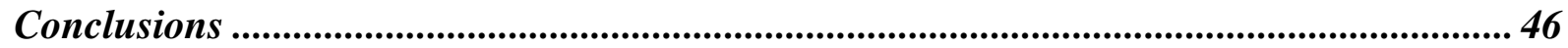

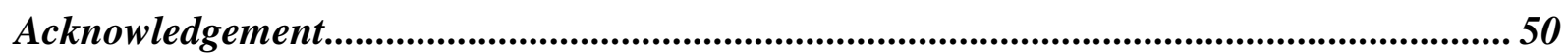

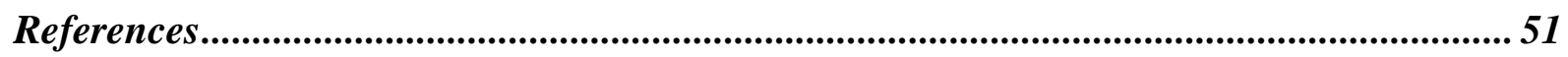




\section{Introduction}

\section{The extracellular space and its role}

During the evolution of animal cells, cells that specialized in the same task extracellular space are organized by the extracellular matrix (ECM) into the tissue ${ }^{1}$. Around the extracellular matrix, the boundary formed around the various functions of the extracellular matrix. ECM helps cell development and provides mechanical protection. Through the network of the basic structure of the matrix, which consists of macromolecular elements produced by surrounding cells, it is possible to communicate between cell migration and tissues ${ }^{2}$. The high level of organization of ECM accomplished by proteins produced by the surrounding cells and delivered to the cellular space. These proteins, most water-insoluble collagen, and collagen-like proteins produced and water-soluble proteoglycans ${ }^{3}$. The connection can make between the cells and the matrix in two ways to create tissues. One possibility is that a direct cell-cell relationship established, and the other is indirectly using ECM's macromolecular system to form a relationship. Between relatively loose and loosely linked cells, a significant proportion of ECM observed, while this proportion is significantly lower among the closely related cells and tissues. Tissues are cell communities that have functions that go far beyond what any cell type can achieve. Healthy tissues consist of proper mixing of cells, and the cells in it must be positioned correctly and divide in the right proportions. To coordinate their function, organization, and mortality and division rates, tissue cells are continually working and responding to signals from each other and the surrounding $\mathrm{ECM}^{4}$. Last but not least, the ECM binds a number of growth factors and hormones that concentrate abundant signals cells that are in contact with it. Cellular responses to these signals are generated directly by the cell-ECM interaction.

Basement membranes (BMs) are 80-100 nm thick, sheet-like extracellular matrices located beneath epithelial and endothelial cells of muscle, nervous, vascular, and adipose tissues 5,6. Without BM integrity, skeletal muscle stability cannot achieve. BMs contain smaller and larger proteins, including type IV collagen, laminin, fibronectin, elastin, nidogen, nidogen, agrin, perlecan, and integrins $5,7,8$. In the latter case, the narrow ECM fills the basal membrane (membrana basalis) and carries almost a limiting function. The extracellular protein 
composition of the basal membrane divided into two main parts, which manifested in the basal lamina (lamina basalis) and retinal lamina (lamina reticularis). In the basal lamina, non-fibrillar collagens, non-collagen-like glycoproteins, and proteoglycans found. In the reticular lamina, fibrillar collagens embedded in proteoglycans found. The basal membrane is found almost everywhere in the human body, encircles striated muscle fibers, binds smooth muscle fibers, and also epithelium and endothelial cells. It can found in the eyepiece, eye corneal cornea, and inner ear.

In mammals, collagens are the most abundant proteins ${ }^{9}$. Its location is in the extracellular matrix, where most of them form a supramolecular unit. Each member of the collagen superfamily contains a triple-helical motif, which ratio for between $\sim 10 \%$ and $96 \%$ of the total molecule ${ }^{10}$. Collagens include three polypeptide chains, called alpha chains, which can form homo- or heterotrimers. Triple helices can form fibrils, networks, two- or threedimensionally highly organized structures.

The main component of the mammalian basement membrane is type IV collagen, which is capable of networking ${ }^{11}$. The epithelium and endothelium, located at the base of the cells, act as a barrier between tissue sections. Due to the abundance of binding partners, this forms the backbone of the basement membrane. Consequently, type IV collagen is not only essential structural collagen of the basement membrane but also has a key signaling potential and is therefore important for a variety of physiological and pathological functions. In mammals, type IV collagens contain six isomeric protein chains, designated $\alpha 1$ (IV) to $\alpha 6$ (IV) ${ }^{12}$. Each type IV collagen molecule is a heterotrimer consisting of three $\alpha$ chains. C-terminal $\mathrm{NC} 1$ domain interactions initiate the assembly of heterotrimeric chains. The collagen domains then folded into triple helices. Currently, three types of type IV collagen heterotrimers are known: $\alpha 1$ (IV) 2 - $\alpha 2$ (IV), $\alpha 3$ (IV) - $\alpha 4$ (IV) - $\alpha 5$ (IV) and $\alpha 5$ (IV) 2 - $\alpha 6$ (IV) ${ }^{13}$.

\section{The collagen superfamily}

The key to the diversity of multicellular animals is the ability of cells to connect and form organs and tissues. This process required a supramolecular contact to hold them together. These molecular "adhesives" are collagens. Each collagen protein contains a triple helix that is part of the supramolecular complex ${ }^{11}$. As a result of the interconnection in a defined way, a 
regular network structure is obtained. The primary function of this mesh is to provide a support structure and to provide considerable flexibility and resistance to organs and tissues. As part of the ECM and the BM, they also play a crucial role in cell migration, cell adhesion, regeneration, and signaling. The collagen superfamily is a very complex family, including the 28 known collagen and collagen-like proteins, into nine major families ${ }^{14}$. All collagen proteins share a common structural feature but have at least a triple-helical domain. Collagen is found throughout our bodies. Different types of organs and tissues display their characteristic collagen network. They make up the cartilage, the vitreous body in the eyes, and can also be found in the vascular walls, the lungs, kidneys, and the basement membrane ${ }^{15}$. In my dissertation, I want to deal more with type IV collagen.

\section{Location of type IV collagen genes in the genome}

Type IV collagen chain proteins are encoded by six genes in the human genome. These genes are located in three pairs on different chromosomes in a head-to-head orientation. The COLAA1-COLAA2 genes are located on the q34 region of chromosome 13, the COLAA3COLAA4 genes are located on the q36.3 region of chromosome 2, and the COL4A5-COLAA6 genes are located on the q22.3 region of chromosome X. Fruit fly (Drosophila melanogaster) has only two collagen genes. These col4al-col4a2 genes are located in the $25 \mathrm{C}$ band of chromosome 2, also in head-to-head orientation relative to one another. The protein products of these genes, when linked together, form the major constituent of the basement membrane, complementing with other proteins and helping the cells to anchor and creating apical-basal polarity ${ }^{16}$. Any disruption of the type IV collagen network, due to its systemic nature, destabilizes the BM, resulting in tissue dysfunction.

\section{Biosynthesis, modification and molecular structure of type IV collagen}

The structure of type IV collagen chains is unique. Individual collagen chains are called monomers. The protomer is formed by the convergence of three pieces of monomers in a specific direction. Protomers can only connect to their head-to-head or tail-to-tail regions, creating a collagen network, which is due to its shape, is referred to in the literature as a "Wire mesh". This process requires the activity of several enzymes that are bound to different 
organelles. Collagen mRNAs are bound to the ribosome on the endoplasmic reticule. The resulting proteins are polypeptide chains, consist of 1500-1800 amino acids with specific repetitive sequences. This sequence shows Glycine-X-Y repeats. All third amino acids are generally glycine, which is the smallest space-consuming amino acid and plays an essential role in folding. Typically, amino acid $\mathrm{X}$ is proline, and $\mathrm{Y}$ is hydroxyproline. Post-translational modifications (glycosylation, hydroxylation, phosphorylation) are required for the formation of monomers of the correct structure. Three major domains are discrimination from each other in the resulting proteins.

The $\mathrm{C}$-terminus of the monomer is called the $\mathrm{NC} 1$ domain, and the $\mathrm{N}$-terminus is the 7S domain (Figure 1) ${ }^{17}$. The Glycine-rich triple-helical region in the middle of the polypeptide chain is comprised of about 1400 amino acids. The three monomeric NC1 domains are joined by initiating protomer formation. This is when the disulfide and hydrogen bridges are formed, which stabilize the structure. The secretion of the protomers occurs by transport vesicles into the extracellular space where $\mathrm{N}$ and $\mathrm{C}$ peptidases cleave $1-1$ of the $\mathrm{NC} 1$ and $7 \mathrm{~S}$ domains. This produces active propepid. The propeptides are capable of binding to the collagen network covalently cross-linked by the lysine oxidase enzyme, stabilizing the final supramolecular structure of the collagen network.

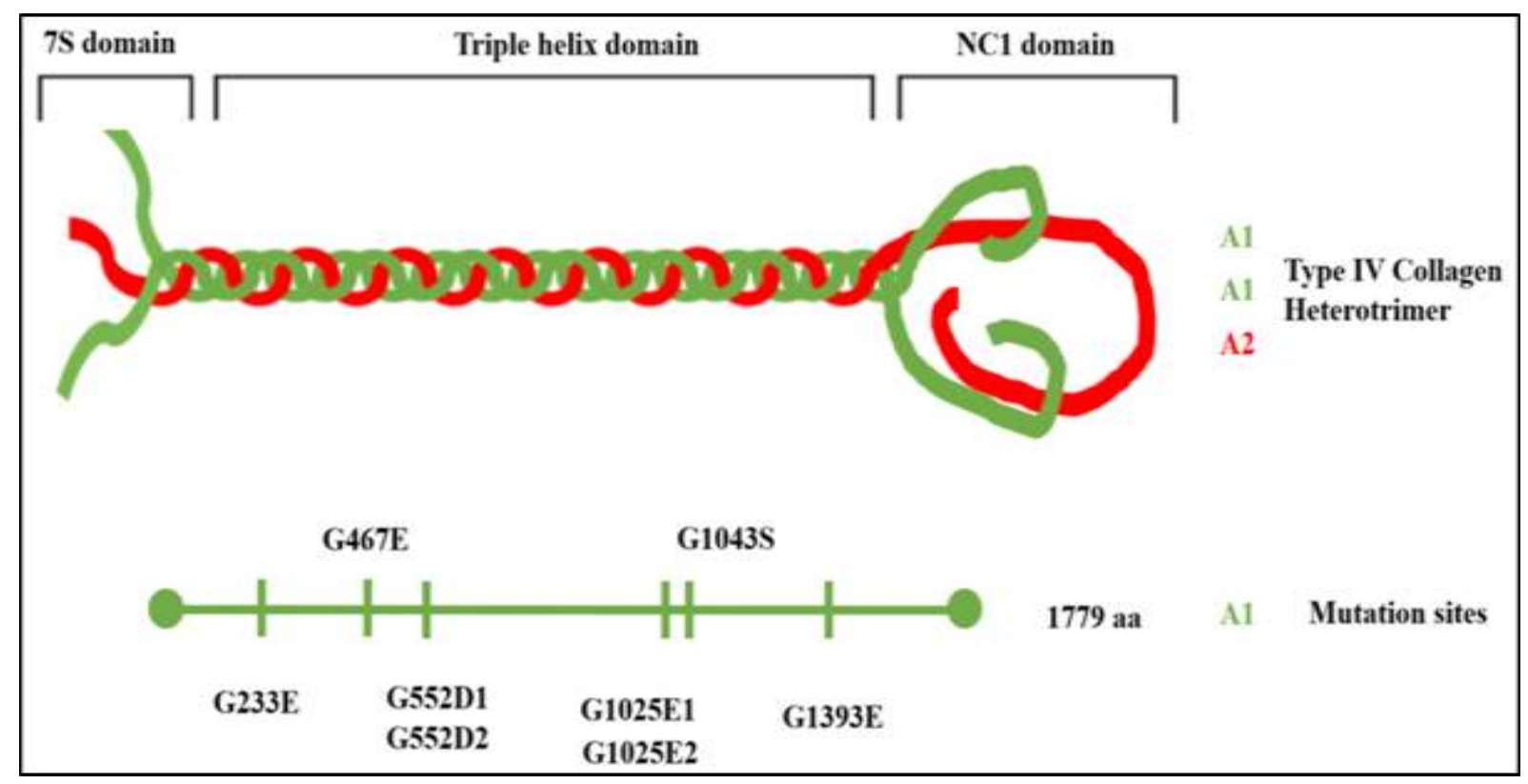

Figure 1. Triple-helical model of type IV collagen trimer with [COLAAI] ${ }_{2} C O L 4 A 2$ composition (top). Distribution of the mutation sites on the COLAA1 protein (bottom). 


\section{The types of network created by type IV collagen}

There are 6 different types of type IV collagen in the human body, which in various combinations can connect and thus create unique networks. The presence of extraordinary collagen networks shows organ and tissue specificity ${ }^{18}$. The triple helix network type $(\alpha 1)_{2}(\alpha 2)$ - $(\alpha 1)_{2}(\alpha 2)$ is found in the basement membrane. This is the ancient type of type IV collagen mesh that already appears in sponges and is most widely distributed in the animal world. The composition of the following BM network type in the human body is the $(\alpha 3)(\alpha 4)(\alpha 5)-(\alpha 3)$ $(\alpha 4)(\alpha 5)^{19}$ triple helix. It occurs in the lungs, cochlea, renal glomerular basement membrane (GBM), eyes, and testes ${ }^{20}$. If rise an error in this network is of particular importance in Alport's syndrome and Goodpasture's syndrome. The last noteworthy triple-helical network type the $(\alpha 1)_{2}(\alpha 2)-(\alpha 5)_{2}(\alpha 6)$ is found in Bowman's capsule, esophagus, smooth muscle, and skin. Network types in organs and tissues may change in the lifetime in rodents, dogs, and humans. In humans the $(\alpha 1)_{2}(\alpha 2)-(\alpha 1)_{2}(\alpha 2)$ collagen network in the renal GBM is exchanged for $(\alpha 3)$ $(\alpha 4)(\alpha 5)$ - ( $\alpha 3)(\alpha 4)(\alpha 5)$ by day 75 of embryonic development. The collagen mesh $(\alpha 1)_{2}(\alpha 2)$ $(\alpha 1)_{2}(\alpha 2)$ in the Bowman's capsule is also changed to $(\alpha 1)_{2}(\alpha 2)-(\alpha 5)_{2}(\alpha 6)$ by day 150 of embryonic development. This structural change in ontogeny is necessary for the maturation of the kidneys ${ }^{21}$. To date, only the $(\alpha 1)_{2}(\alpha 2)-(\alpha 1)_{2}(\alpha 2)$ triple-helical type IV collagen network is present in Drosophila melanogaster. If any of the polypeptide chains are disturbed, it causes a systemic illness throughout the body.

\section{Type IV collagen plays a central role in muscle development and structure formation}

The most important contractile apparatus of the body is muscle tissue. Within this, skeletal muscles play an essential function. The skeletal muscle is made up of heterogeneous tissues that are $30-38 \%$ of the human body ${ }^{22}$. Its most important task, maintaining posture, movement, and breathing. Muscle consists of individual fibers, which are organized into muscle bundles, and the related bundles form the muscles. The extracellular matrix plays an essential role in the formation of muscle structure. The ECM assists in the production of mammalian muscle structure at three different tissue levels as an intramuscular connective tissue. At its most internal level, it forms the endomysium, which surrounds the individual muscle fibers. At 
the middle level, it forms perimysium that surrounds the muscle bundles. At the very outer level, it forms the epimysium that surrounds the entire muscle. Type IV collagen is the most significant molar component of mammalian muscle BM, accounting for up to $10 \%$ of the total muscle mass. The muscle basement membrane is mostly composed of type IV collagen. BM binds several linker molecules to the sarcolemma (muscle fiber membrane) and cells. The most important of these are the proteoglycans, which help to build the regular collagen network. Glycoproteins (laminin, integrin, fibronectin, dystroglycan) are also significant factors. Laminins play a role in muscle cell division, movement, and morphology formation. The fibronectins help the adhesion of fibroblasts. Integrins have a linking role in facilitating cell attachment to BM, and they also have many other functions from signal transduction processes to cell growth and differentiation. The passive strain on muscles is performed by the ECM and plays an important role in maintaining normal muscle structure. If the major structural components of the ECM are damaged, the whole muscle system may be affected. Some examples include but are not limited to. A point mutation in the col4al gene (col4al ${ }^{G 498 V}$ ) causes morphological defects whose characteristics it shares with inherited muscle dystrophy ${ }^{23}$. Duchenne's muscular dystrophy is caused by the abnormal function of the dystrophin protein ${ }^{24,25}$. Congenital muscle dystrophy ${ }^{26}$ may occur in the case of laminin failure.

\section{Diseases associated with type IV collagen mutations in human pathology}

\section{Alport syndrome}

Alport syndrome (AS) is inherited progressive kidney disease ${ }^{27}$. The main characteristic features of the disease are structural defects of the glomerular basement membrane leading to end-stage renal failure (ESRD), sensorineural hearing loss, and ocular abnormalities, hematuria, and proteinuria ${ }^{28}$. The proportion of affected patients compared to healthy patients is approximately 1: 5000. AS is caused by mutations in the type IV collagen gene in the $\alpha 3, \alpha 4, \alpha 5$ chains. Based on pathological and genetic characteristics, AS is divided into three groups. The first and most common group is X-linked Alport syndrome (XLAS) ${ }^{29}$, which occurs in $80 \%$ of cases. It got its name from the fact that the affected COL4A5 gene is located on the $\mathrm{X}$ chromosome, so men are more affected than women because of the mosaicism caused by X chromosome inactivation (XCI). The other two genes, the COL4A3 and COL4A4 
autosomal genes, are located on chromosome 2. The different two types of AS are Autosomal Recessive Alport Syndrome (ARAS) ${ }^{30}$, which occurs in $15 \%$ of cases, and the third type is Autosomal Dominant Alport Syndrome (ADAS), which occurs in 5\% of cases. The pathological background of the disease is that the triple-helical type IV collagen chains in the GBM $((\alpha 3)(\alpha 4)(\alpha 5)-(\alpha 3)(\alpha 4)(\alpha 5))$ cannot be linked, therefore the damaged filtration barrier is unable to perform a function that the collagen network $((\alpha 1) 2(\alpha 2)-(\alpha 1) 2(\alpha 2))$ of the Bowman's capsule cannot counterbalance ${ }^{31}$. There is currently no sure long-term treatment for the disease. Patients may gain some time with dialysis and kidney transplantation.

\section{Goodpasture's syndrome}

Goodpasture's syndrome (GS), also known as anti-glomerular basement membrane disease ${ }^{32}$, is a rare organ-specific autoimmune disease that affects the kidneys and lungs. Another characteristic is hemorrhage in the lungs, which can be lied to in life-threatening conditions. GS incidence is one per million in a year. The disease typically affects white people and leads to acute renal failure if left untreated. The age distribution is bimodal, it has a double peak. The first peak occurs in the 20s and 30s and usually affects young men. The second peak in the $60 \mathrm{~s}$ and $70 \mathrm{~s}$ is more pronounced in older women. The etiology of GS is currently unknown. Regarding its immunopathogenicity, it is a type IV collagen disease in which the body produces autoantigens (Goodpasture antigens) ${ }^{33}$ primarily against the $\mathrm{NC} 1$ domain of the $\alpha 3$ chain but also to a lesser extent against the $\mathrm{NC} 1$ domain of the $\alpha 5$ chain ${ }^{34}$. An aggravating environmental factor is smoking and inhaling hydrocarbons. Autoantibodies attack the NC1 domains of the $\alpha 3$ and $\alpha 5$ chains of the triple-helical network of type IV collagen constituting the basement membrane. This type of triple helix combination ( $\alpha 345)$ is typically present in the lungs and GBM. Linking the NC1 domain to another NC1 domain stabilizes the basement membrane structure. As a result of the immune response, this linkage is broken down, and the two $\mathrm{NC} 1$ domains are separated, causing the destabilized collagen network to be a blockage on the basement membrane and eventually creating a life-threatening condition. There is currently no known cure for GS. The therapy to be applied consists of three steps. The first one is plasmapheresis. This is followed by immunoadsorption, during which the harmful autoantibodies are removed. This is combined with medication and immunosuppressive therapy but eventually requires kidney transplantation. 


\section{HANAC syndrome}

HANAC syndrome is an acronym for Hereditary Angiopathy with Nephropathy, Aneurysms, and Muscle Cramps, a basement membrane disease associated with autosomal dominant COL4A1 gene mutations ${ }^{35}$. By its very nature, it has a systemic appearance. Genetic causes include missense mutations in the COL4A1 gene, which mainly affect glycine amino acids ${ }^{36}$. Amino acid substitutions of the absolutely conserved glycines in the collagenous helix can alter the steric structure of the protein and prevent the attachment of other proteins by changing their recognition sequences. This results in a reduced $\mathrm{ECM}^{37}$. Because of its systemic nature, it causes pathological conditions throughout the body. Symptoms of HANAC syndrome are usually associated with complete basement membrane defects, with a variable combination of small vessel brain disease, Raynaud's phenomenon, and nephropathy ${ }^{38}$. 


\section{Aims}

In my research, I was curious about how mutations in col4al affect the function of selected organs. For this work, I chose Drosophila melanogaster as the model organism because it carries only two col4a genes. As a result of our previous work, we have conditional col4al mutants in which we have also been able to describe its effect on the development of striated muscles ${ }^{9}$.

In my thesis, I summarize my observations, which intended to examine how they changed as a result of col4al mutations:

- To examine changes in the amount of COL4A1 protein in mutant animals to obtain a more comprehensive picture of the two different phases of development at different temperatures.

- Immunofluorescent images of the common oviduct of Drosophila melanogaster to show changes in sarcomere structure due to the col4al mutation.

- To investigate additional extracellular proteins related to COL4A1 protein in the midgut at different stages of development.

- To confirm the harmful systemic nature of the mutation, we will examine the changes in the cytoskeletal-extracellular axis proteins of Malpighian tubules.

- Finally, we were interested in how the morphology of mitochondria changes as a result of mutations in the Malpighian tubules. 


\section{Materials and methods}

\section{List and maintenance of Drosophila strains}

Eight dominant temperature-sensitive (DTS) mutant $F$ strains were available in our lab. The mutations are on the chromosome 2 , and each is a point mutation affecting one glycine in the helical domain of col4al chain. The strains were maintained using a balancer chromosome (chromosome 2). There are two markers on the balancer chromosome that can be used to track our mutations in crosses. One marker is curly $(C y)$, which produces a curly wing. Another marker is Rough Eye (Roi), which is a dominant mutation and causes rough eye surface owing to irregular assembly of the ommatidia.

\section{The col4a1 mutant strains used in this study (with old and new names)}

- $\quad$ Oregon $R$ (wt, wild-type): Red colored and smooth surface with a straight wing.

- a-30 / CyRoi (new notation: col4al ${ }^{G 233 E}$ ): Red-colored and coarse-grained eye, curly wings. The type IV collagen protein is mutated in the 233th position of the amino acid sequence (G / E) of COL4A1 propeptid.

- w, DTS-L2 / CyRoi (new notation: col4al ${ }^{G 552 D 1}$ ): White color and coarse surface eye, curly wings. The type IV collagen protein is mutated at position 552 of the amino acid sequence (G / D) of COL4A1 propeptid.

- DTS-L3 / CyRoi (new notation: col4a1 ${ }^{G 552 D 2}$ ): Red-colored and coarse-faced eyes, curly wings. The type IV collagen protein is mutated at position 552 of the amino acid sequence $(\mathrm{G} / \mathrm{D})$ of COL4A1 propeptid.

- DTS-L4 / CyRoi (new notation: col4a1 ${ }^{G 1025 E 1}$ ): Red-colored and coarse-to-surface eyes, curly wings. The type IV collagen protein is mutated in the 1025th position (G / E) of the amino acid sequence.

- DTS-L5 / CyRoi (new notation: col4al ${ }^{\text {G1025E2 }}$ ): Red-colored and coarse-grained eye, curly wings. The type IV collagen protein is mutated in the 1025th position (G / E) of the amino acid sequence. 
- DTS-L10 / CyRoi (new notation: col4al ${ }^{\text {G1043S }}$ ): Red-colored and coarse-grained eye, curly wings. The type IV collagen protein is mutated in the amino acid sequence at position 1043 (G / S).

- b-9 / CyRoi (new notation: col4al ${ }^{G 467 E}$ ): Red-colored and coarse-grained eye, curly wings. The type IV collagen protein is mutated at position 467 of the amino acid sequence $(\mathrm{G} / \mathrm{E})$.

- b-17 / CyRoi (new notation: col4al ${ }^{G 1393 E}$ ): Red-colored and coarse-faced eyes, curly wings. The type IV collagen protein is mutated in the amino acid sequence at position 1393 (G / E).

The used mitochondria labelled mutant strain

- $\quad \mathrm{w} / \mathrm{w},+/+, \mathrm{P}\left\{\mathrm{w}^{*}\right.$ sqh-EYFP-Mito $\} / \mathrm{P}\left\{\mathrm{w}^{*} \text { sqh-EYFP-Mito }\right\}^{41}$

Wild-type Oregon flies and coltal mutant stocks were maintained at $20{ }^{\circ} \mathrm{C}$ and $29{ }^{\circ} \mathrm{C}$ on yeast-cornmeal- sucrose-agar food, consisting of nipagin to prevent fungal infection. The mutant stocks were kept heterozygous over the CyRoi balancer chromosome.

\section{Tissue dissection}

Malpighian tubules, gut and common oviduct were removed under carbon dioxide anaesthesia from adults that were grown at both the permissive and restrictive temperature for 14 days. Dissected organs were fixed in 4\% paraformaldehyde dissolved in phosphate buffered saline (PBS) for $10 \mathrm{~min}$, washed three times in PBS, permeabilized for $5 \mathrm{~min}$ in $0,1 \%$ Triton $\mathrm{X}$ dissolved in PBS and washed three times in PBS. Blocking was achieved in 5\% BSA dissolved in PBS for 1 hour and washed three times in PBS.

\section{Staining methods, antibodies}

Nuclei in the dissected organs were counter-stained by $1 \mu \mathrm{g} / \mathrm{ml} \mathrm{4}$,6-diamino-2phenylindole (DAPI) in $20 \mu \mathrm{l}$ PBS, $12 \mathrm{~min}$ in the dark. F-actin was stained by 1 unit Texas Red $^{\mathrm{TM}}$-X Phalloidin (ThermoFisher) in $20 \mu \mathrm{l}$ PBS for $20 \mathrm{~min}$. We stained a-mannopyranosyl and a-glucopyranosyl residues as a membrane marker by $2,8 \mu \mathrm{g} / \mathrm{ml}$ Concanavalin A, Alexa Fluor $^{\mathrm{TM}} 594$ Conjugate (ThermoFisher) in $20 \mu \mathrm{l}$ PBS for 1 hour. We used $1 \mu 1$ mouse 
monoclonal Anti-3-Nitrotyrosine [39B6] (Abcam) antibody to 3-nitrotyrosine for 1 hour and stained 4-hydroxynonenal conjugates by $1 \mu 1$ mouse monoclonal Anti-4-hyroxynonenal antibody (Abcam) for 1 hour. $1 \mu \mathrm{l}$ F(ab') 2-Goat Anti-Mouse IgG (H+L) Cross Adsorbed Secondary Antibody, Alexa Fluor 488 (ThermoFisher) in $20 \mu \mathrm{l}$ PBS for 1 hour, or $1 \mu 1$ Goat Anti-Mouse IgG (H+L) Cross Adsorbed Secondary Antibody, Alexa Fluor 350 in $20 \mu 1$ PBS for 1 hour. Integrin alpha PS (mouse) and -kettin (rat) antibodies (Abcam) were used in $20 \mu 1$ PBS for 1 hour. Mouse antibody against Drosophila COL4A1 were raised by Creative Laboratory Ltd., Szeged, Hungary. Primary mouse antibodies were visualized by $1 \mu 1$ F(ab') 2Goat Anti-Mouse IgG (H+L) Cross Adsorbed Secondary Antibody conjugated with Alexa Fluor 488 (ThermoFisher) in $20 \mu 1$ PBS for 1 hour or $1 \mu 1$ Rabbit anti-Rat IgG (H+L) Secondary Antibody, Alexa Fluor ${ }^{\circledR} 594$ conjugate in $20 \mu 1$ PBS for 1 hour. All immunostaining was performed in $20 \mu \mathrm{l}$ PBS for 1 hour.

\section{Quantitative fluorescence confocal microscopy}

Photomicrographs of the Malpighian tubules, intestinal tract and common oviduct were generated by confocal laser scanning fluorescence microscopy (Olympus Life Science Europa $\mathrm{GmbH}$, Hamburg, Germany). Microscope configuration was the following: objective lens: UPLSAPO 60x (oil, NA: 1.35); sampling speed: $8 \mu \mathrm{s} /$ pixel; line averaging: 2x; scanning mode: sequential unidirectional; excitation: $405 \mathrm{~nm}$ (DAPI), $543 \mathrm{~nm}$ (Texas Red) and $488 \mathrm{~nm}$ (Alexa Fluor 488); laser transmissivity: 7\% were used for DAPI, $42 \%$ for Alexa Fluor 488 and 52\% for Texas Red.

Quantitative evaluation of the fluorescence light intensities of the $543 \mathrm{~nm}$ Texas Red signals was achieved by the FLUOVIEW FV1000 program of Olympus, system version 4.2.1.20. We generated $\sim 20$ independent images for each wild-type control and mutant samples thus analyzing over 400 photomicrographs. Statistical analysis was carried out by analyzing raw confocal fluorescent light intensities. Signal amplification was applied in images for morphologic analysis.

\section{Western blot analysis}

Western blotting is one of the most commonly used techniques in molecular biology and proteomics ${ }^{42,43}$. 
Sample preparation:Isolation of polypeptides from larvae and adults in the Eppendorf tube and pass the (2x) Laemmli solution (0.5M Tris- $\mathrm{HCl}(\mathrm{pH} 6.8) 2.5 \mathrm{ml} ; 10 \%(\mathrm{w} / \mathrm{v})$ SDS 4.0 $\mathrm{ml}$; $0.1 \%$ Bromophenol blue $0.5 \mathrm{ml}$; Glycerol $2.0 \mathrm{ml}$; 2-mercaptoethanol $0.5 \mathrm{ml}$; make up to 10 $\mathrm{ml}$ with distilled water), then treat the animals with potter and cook for 10 minutes and centrifuge for 5 minutes at $14,000 \mathrm{rpm}$. Concentrations of protein solutions isolated were measured according to Bradford ${ }^{44}$ and $10 \mu \mathrm{g}$ of proteins were loaded in each lane.

Preparation of $6 \%$ separating gel (acrylamide gel): $\mathrm{H}_{2} \mathrm{O} 5.3 \mathrm{ml} ; 30 \%$ acrylamide mix $2.0 \mathrm{ml}$; 1.5M Tris (pH 8.8) $2.5 \mathrm{ml} ; 10 \%$ SDS $0.1 \mathrm{ml} ; 10 \%$ APS (ammonium persulfate) $0.1 \mathrm{ml}$; TEMED $0.008 \mathrm{ml}$.

Making the $6 \%$ top gel or collecting gel (stacking gel): $\mathrm{H}_{2} \mathrm{O} 1.4 \mathrm{ml} ; 30 \%$ acrylamide mix $0.33 \mathrm{ml}$; 1.0M Tris (pH 6.8) $0.25 \mathrm{ml} ; 10 \%$ SDS $0.02 \mathrm{ml}$; $10 \%$ APS $0.02 \mathrm{ml}$; TEMED 0.002 $\mathrm{ml}$.

The running buffer has $\sim \mathrm{pH} 8.3$ which is self-priming (Tris base $2.9 \mathrm{~g}$; Glycine $14.4 \mathrm{~g}$; SDS $1 \mathrm{~g}$; make up to $1 \mathrm{~L}$ with distilled water. When the unit is assembled at $100 \mathrm{~V}$, it runs about 1.5 hours.

Blotting: The gel is soaked in the transfer buffer $(6.04 \mathrm{~g}$ Tris; $28,8 \mathrm{~g}$ glycin; $200 \mathrm{ml}$ methanol; $1,6 \mathrm{~L} \mathrm{ddH}_{2} \mathrm{O}$ ) for 15 minutes. Pre-sterilization of the Whatmann 3M filter paper was followed by 15 minutes of soaking in the buffer. PVDF filter was placed in methanol for 1 minute and soaked for 15 minutes in the buffer. Blotted at +4 degrees to avoid warming up or on ice (200 mA, 30 minutes, 55 V). Filter check stain with Ponceau S (Ponceau S 0,1\% (w/v) $0.05 \mathrm{~g} ; 5 \%$ acetic acid $2.5 \mathrm{ml}$; make up to $50 \mathrm{ml}$ with distilled water), then washes with water. Control the gel with Coomassie Blue (50\% methanol; 0.05\% Coomassie Brilliant Blue R; 10\% acetic acid; $40 \% \mathrm{H}_{2} \mathrm{O}$ ) to see how much protein left on the gel. Washing with Destaining Buffer (5\% methanol; $7 \%$ acetic acid; $88 \% \mathrm{H}_{2} \mathrm{O}$ ).

Blocking:1x TBS/Tween 20, ratio: 1000:1, 3\% fat free milk powder (50 ml TBS, $50 \mu 1$ Tween 20, 2,5 g milk powder), or BSA 3\%. (10x TBS receipt: 10x TBS; $60.6 \mathrm{~g}$ Tris; $116.9 \mathrm{~g}$ $\mathrm{NaCl}$; make up to $1 \mathrm{~L}$ with distilled water ( $\mathrm{pH}$ 7.4). This was kept at 4 Celsius overnight or shaken for 1 hour at room temperature. 
Applying antibodies for Western blot: The Drosophila polyclonal anti-collagen type IV a1 antibody (bleed IV, rabbit), recognising both the $\mathrm{N}$ - and C-termini of the Drosophila COL4A1 protein was a generous gift of Prof. J.H. Fessler (UCLA, USA). Anti-rabbit mouse monoclonal antibody (Sigma) conjugated with peroxidase served as secondary antibody. As a protein expression control, alpha-glycogen phosphorylase was detected by polyclonal rabbit antibody (Santa Cruz Biotechnology); anti-rabbit antibody, conjugated with peroxidase, allowed detection of the signal.

The exposure: The two-component Luminol reagent is applied at a ratio of 1 to 1 mixture. Shake for 1 minute at room temperature and apply to the membrane. This may be still in the light. Further operations are carried out in a dark room. Place the film and expose it from $30 \mathrm{mp}$ to 2 hours. Place the film in the developer for 3 minutes, for 1 minute wash with water, about 1-2 minutes of fixation. Calling and fixing solutions are solutions of TETENAL X-ray film. Rinse with clean water.

\section{Statistical methods}

The statistical calculations were performed with Microsoft Office Excel and free R3.5.2 and RStudio-1.1.463.

\section{Elevated Z-disc expressivity in mutants}

The basic statistics were first calculated from the kettin and integrin fluorescence intensity data extracted from the confocal microscope recordings.

T-tests are very robust and therefore powerful than non-parametric probes in the event of a slight violation of normality. Since the variances are not equal, we have chosen Welch's two-sample t-test. During the operations, we compared the mutants to the control strain (Oregon $\mathrm{R})$ at a significance level of $\mathrm{p}=0.05$. The result of the Welch test $(\mathrm{p}=0.003973)$ showed a significant difference.

The graph shows averages of strain values and a standard error as an error band. 


\section{Results and Discussion}

\section{Decreased amount of COLAA1 protein in DTS-L3 mutant at $29{ }^{\circ} \mathrm{C}$}

Protein extracts from Oregon-R and DTS-L3 3rd instar (L3) larvae and adult flies were prepared for Western blot analysis. The larvae incubated at $20^{\circ} \mathrm{C}$ and $29{ }^{\circ} \mathrm{C}$ and blotted with Drosophila polyclonal anti-COL4A1 antibody. At $200 \mathrm{kDa}$, a single band was detected, which corresponded to the previously reported size of the COL4A1 monomer ${ }^{45}$. Wild-type larvae showed faint bands at both temperatures but those were not detectable in mutant larvae. The $200 \mathrm{kDa}$ band was present in wild-type adults at both temperatures and in mutants in the $20{ }^{\circ} \mathrm{C}$ samples but showed a reduced intensity at $29^{\circ} \mathrm{C}$ (Figure 2). For the next analysis of expression levels of extracellular matrix and matrix-associated genes, DTS-L3 adult mutant samples were exposed to $29{ }^{\circ} \mathrm{C}$. Slightly elevated levels of col4al mRNA were found, indicating that the decrease in COL4A1 protein level was not caused by a transcriptional change. Altogether, these results indicate that collagen levels are reduced in the common oviduct of mutant flies incubated at $29^{\circ} \mathrm{C}$, which is likely due to inadequate assembly and deposition of collagen chains.

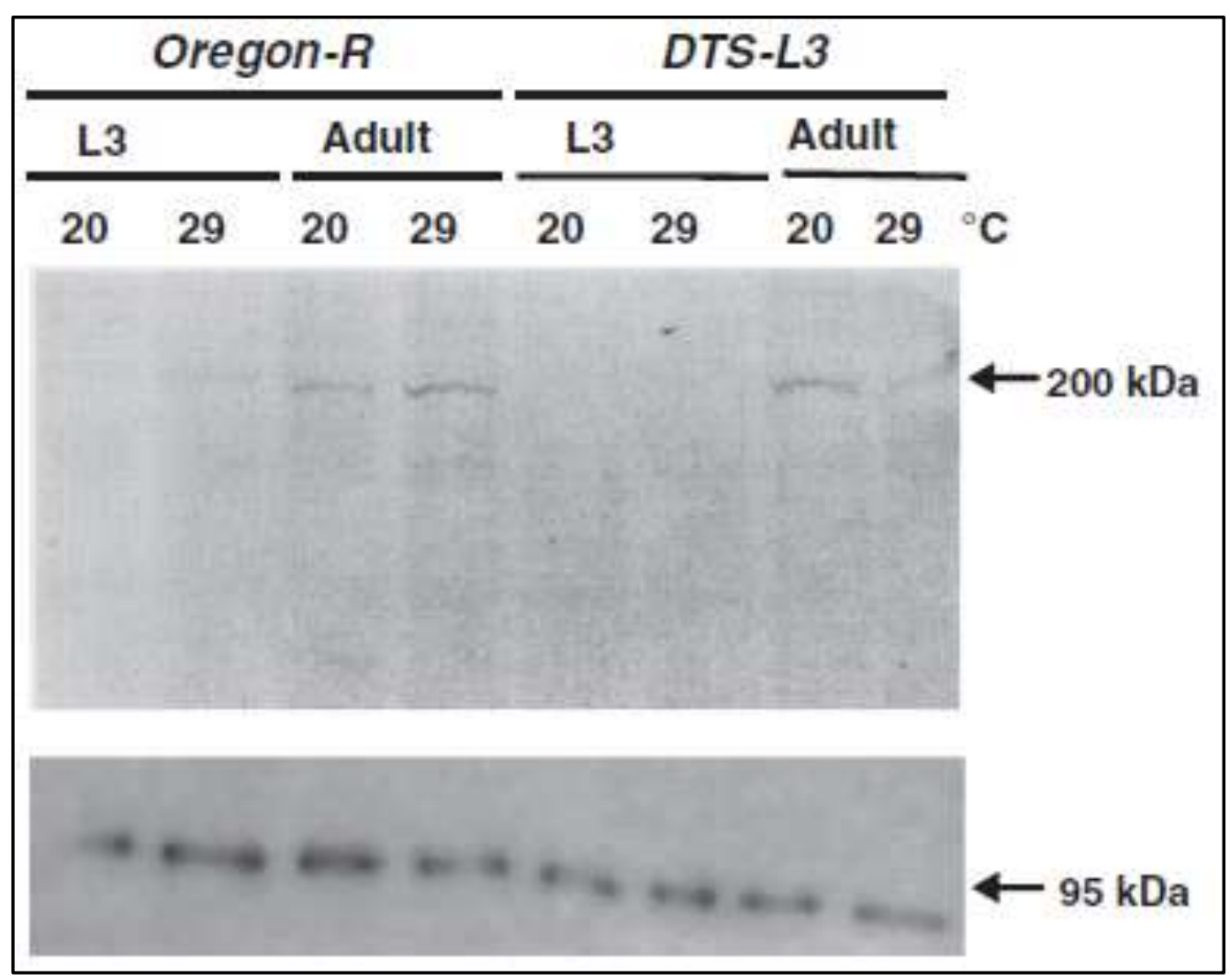


Figure 2. Upper panel: Western blot analysis of COL4A1 in Oregon-R and DTS-L3 mutant protein extracts prepared from larva $(L)$ and adult animals incubated at $20^{\circ} \mathrm{C}$ and $29^{\circ} \mathrm{C}$ using Drosophila polyclonal anti-collagen IVal antibody. The $200 \mathrm{kDa}$ COLAAl band is expressed at low levels in larvae, it is present in normal adult controls, but in the mutant it is not detectable in larval extracts. In adult mutants it is present at $20^{\circ} \mathrm{C}$, but diminished at $29^{\circ} \mathrm{C}$. Lower panel: Protein expression control alpha glycogen phosphorylase.

Col4al/2 ${ }^{-/-}$mice null mutants die in early embryonic development ${ }^{46}$. Recessive null mutant and semi-dominant temperature-sensitive mutants of Caenorhabditis elegans also die during embryogenesis and the intracellularly accumulated mutants a1 (IV) and a2 (IV) chains, indicating defective secretion or assembly of these proteins ${ }^{47}$. In particular human cases of Alport syndrome, type IV collagen has been described, and similar results have been obtained with mutant protein chains. Probably what happened here was that the triple helices did not assemble correctly and not built-into the basement membrane ${ }^{48}$. In contrast, in Drosophila, the development of semi-lethal DTS / + heterozygotes at $29^{\circ} \mathrm{C}$ lacks a sharply distinct lethal phase, suggesting that mutant chains are somewhat assembled in the triple helix and incorporated/deposited in BM. This results in a sub-vital condition, which shows that the basement membrane collagens are slightly different in Drosophila.

Considering the essential function of the basement membrane in most organs and tissues, and the pleiotropic phenotype of the mutants, the Drosophila col4al mutants are remarkably similar to the mammalian COL4A1 and COL4A2 mutations, including myopathy, occurrence of split muscle fibers and non-peripheral nuclei in $\mathrm{Col}_{4 a 1^{ \pm}} /{ }^{4 e x 40}$ mice ${ }^{49}$. In various myopathies and muscle dystrophies, the presence of the central nuclei is a constant feature in the regeneration and degeneration cycles of human and mouse muscle fibers. Mutations in human genes myotubularin ${ }^{50,51}$, myotubularin-related $\quad$ protein $14^{52}$, amphiphysin ${ }^{53}$, and dynamin $2^{54}$ similarly associate with centronuclear myopathies. Drosophila mutants for myotubularin (CG9115; Construct ID: 14121), amphiphysin ${ }^{55,56}$, and dynamin ${ }^{57}$ have been also reported with various degrees of muscle involvement, however, without central nuclei in myofibers. 
Patients with mutations in COLAAI with basement membrane defects and pathological manifestations in various tissues have been reported in numerous clinical genetic studies. Muscle weakness, cramps, high creatine kinase levels ${ }^{38}$, bilateral leg muscle contractures ${ }^{58,59}$ and dystrophy and atrophy of leg muscles ${ }^{60}$ have been noted, strongly supporting muscle manifestation of COL4Al mutations. However, the study of mutant muscle tissue at histological depths, cell-based, and biochemical relevant information is still lacking. Therefore, the essential details of the pathomechanism are unknown. Hence, our Drosophila col4al mutants may serve as a useful model to elucidate the mechanisms underlying COL4A1 mutation results in disturbed muscle fiber-basement membrane interactions, which may result in the manifestation of aberrant muscle function associated with COL4A1-related disorder.

\section{Col4a1 mutations cause sarcomeric disorders in common oviduct muscle tissue}

The common oviduct is surrounded by a single-layer of striated muscle fibers, which is thus very easy to examine in each mutant by fluorescent confocal microscopy. In a normal situation, these muscle fibers present a regular sarcomere structure, as shown in Figure 3.

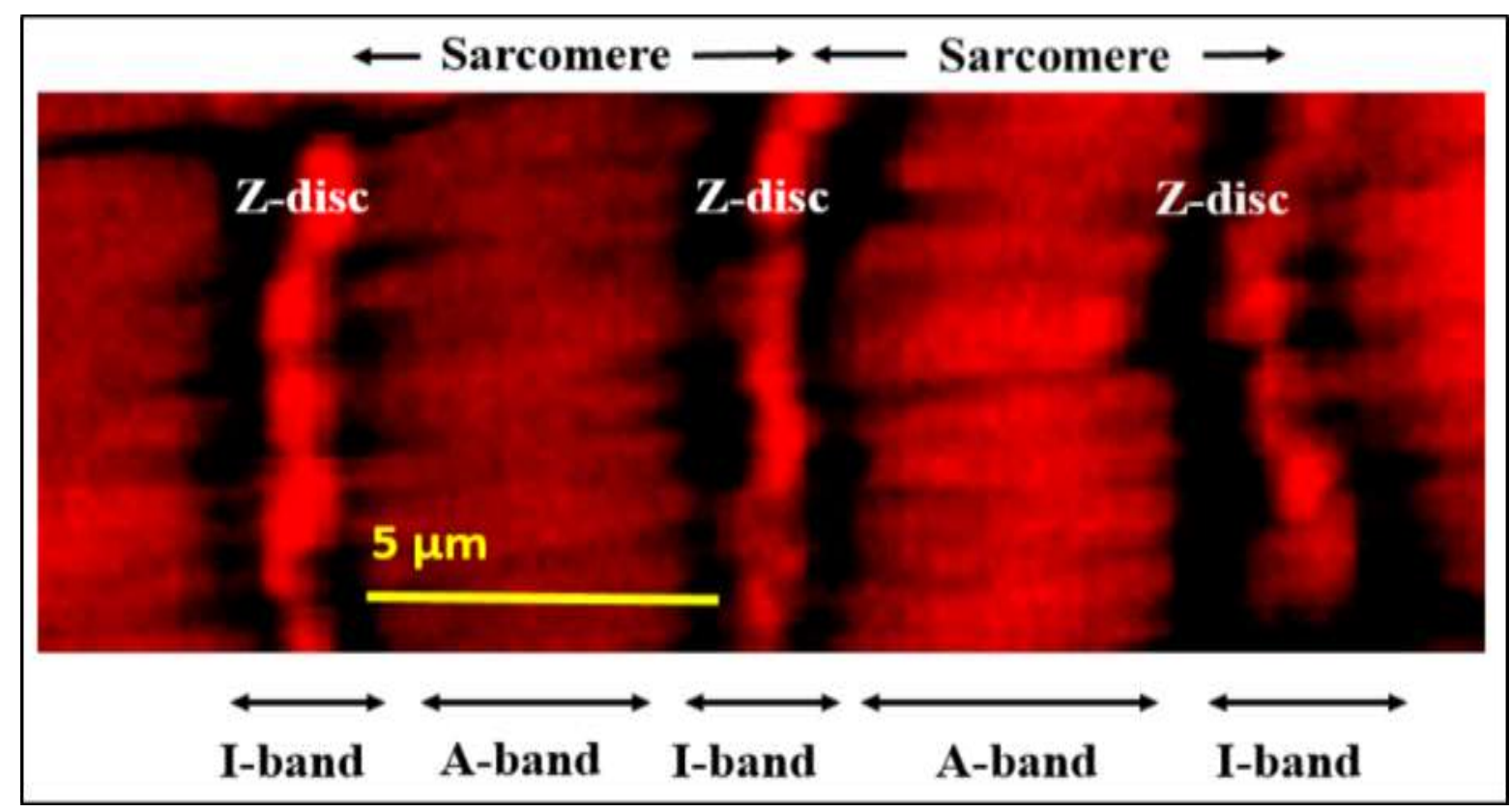


Figure 3. Regular sarcomere units within a common oviductal muscle fiber demonstrated at high resolution by fluorescence confocal microscopy and actin staining by phalloidin.

In the mutants, however, the most well-marked phenotype was the loss of sarcomeres at the restrictive temperature, $29^{\circ} \mathrm{C}$ (Figure 4, B4 through G4), whereas the healthy sarcomere structure and striation was present at both $20^{\circ} \mathrm{C}$ and $29^{\circ} \mathrm{C}$ in wild-type control flies (Figure 3, Figure 4, A1, A4). In mutants at $29^{\circ} \mathrm{C}$, parallel ordered heigh intensity actin staining areas were present within the muscle fibers that widespread over areas further than a single sarcomere, resembling actin stress fibers or excess actin cross-linking (Figure 4, white rectangles in B4 through G4). Beyond these areas, amorphous, all-round actin positive aggregates appeared in the sarcoplasm (Figure 4, white arrows in B4 through G4). One more conspicuous phenotype of the col4al mutants at $29{ }^{\circ} \mathrm{C}$ was the atypical and uneven COL4A1 deposition in the individual muscle fibers (Figure 4, white arrowheads, B5 through G5) while in wild-type controls homogenous COL4A1 staining was present at $29{ }^{\circ} \mathrm{C}$ (Figure 4, A5). In the isoallelic mutants col4al ${ }^{G 552 D 2}$ and col4a1 ${ }^{G 1025 E 2}$, the same COL4A1 staining pattern was recognized as in the other lines of the allelic series (Figure 4). Muscle fibers of the common oviduct in wild-type animals harbor morphologic hallmarks of the striated muscles, present the regular register of I and A bands and sarcomeres are bordered by Z-discs (Figure 1, Figure 4, A1-A6). Therefore, we assume that the compromised sarcoplasmic morphology and irregular COL4A1 staining/localization is a general phenotype of our series of col4al mutations. 


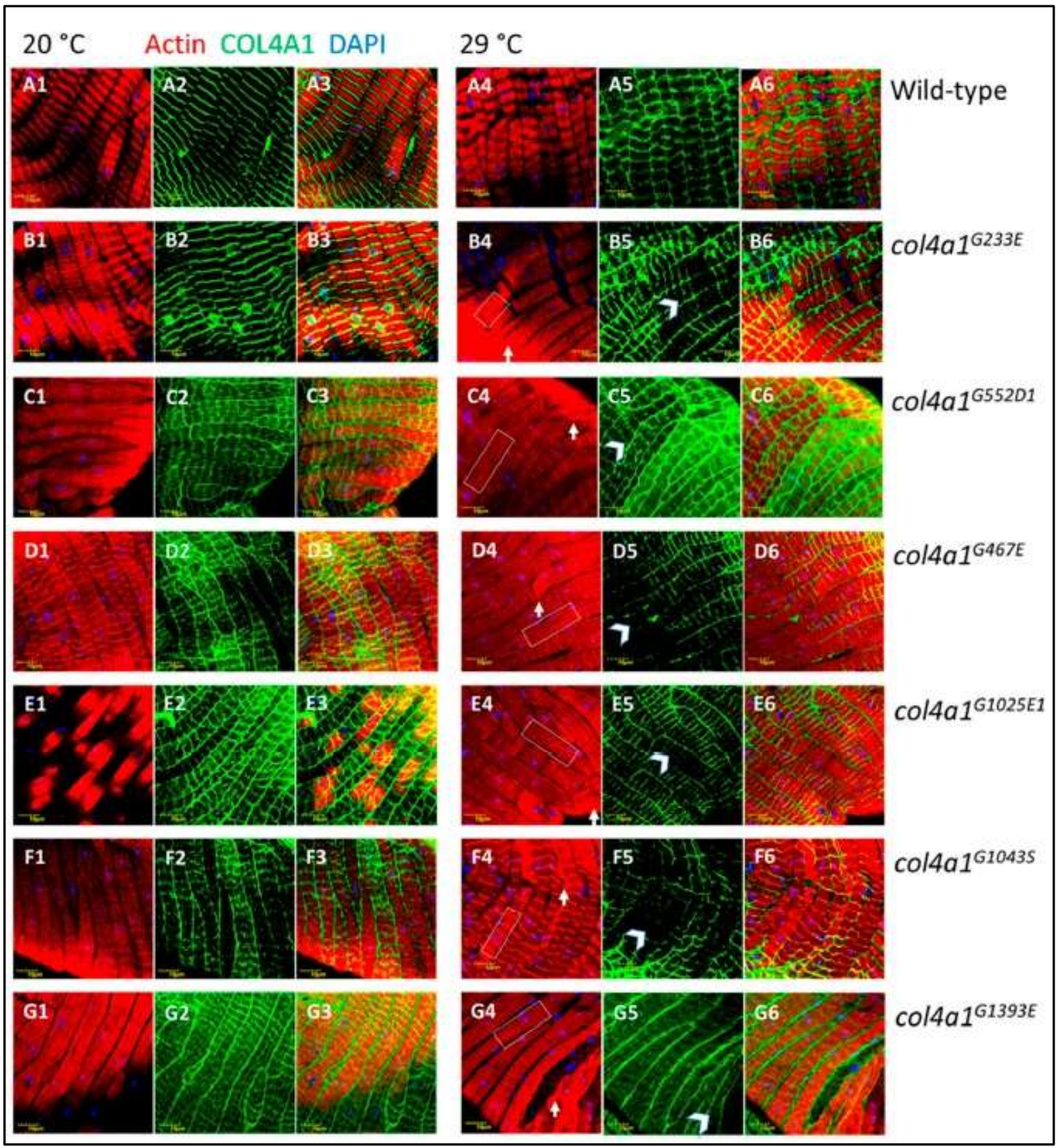

Figure 4. Loss of sarcomeres in col4a1 ${ }^{G 233 E}$, col4a1 $1^{G 467 E}$, col4a1 ${ }^{G 552 D 1}$, col4a1 $1^{G 1025 E 1}$, col4a1 ${ }^{\text {G1043S }}$ and col4a1 ${ }^{\text {Gl393E }}$ mutant lines at $29^{\circ} \mathrm{C}$ (B4 through G4) in comparison with wildtype control (A4). Representative actin bundles (white rectangles in B4 through G4), actin aggregates (white arrows in B4 through G4), uneven COLAAl expression (white arrowheads in B5 through G5). A3 through G3 and A6 through G6: Overlays of actin and COL4A1 staining. Bars, lower left, 10 micrometers. 
Measured by quantitative fluorescence confocal microscopy, the control flies do not modify their actin accumulation in the muscle fibers at permissive or restrictive temperatures. The occurrence of col4al mutation appears to exert a non-consistent influence on the actin content of the muscle fibers at both permissive or restrictive conditions. Statistically significant downregulation of actin synthesis, compared to wild-type control flies, was found in col4al $1^{G 233 E}$, col4al ${ }^{G 552 D 1}$, col4a1 $1^{G 1025 E 2}$ mutants at $20^{\circ} \mathrm{C}$, whereas the col4al ${ }^{G 1393 E}$ mutant synthesized actin at an elevated concentration (Figure 5). Under restrictive conditions, we detect univocal decrease of actin concentration in col4a1 ${ }^{G 552 D 1}$, col4a1 ${ }^{\text {G1043S }}$, and col4al ${ }^{\text {Gl393E }}$ mutants (Figure 5).

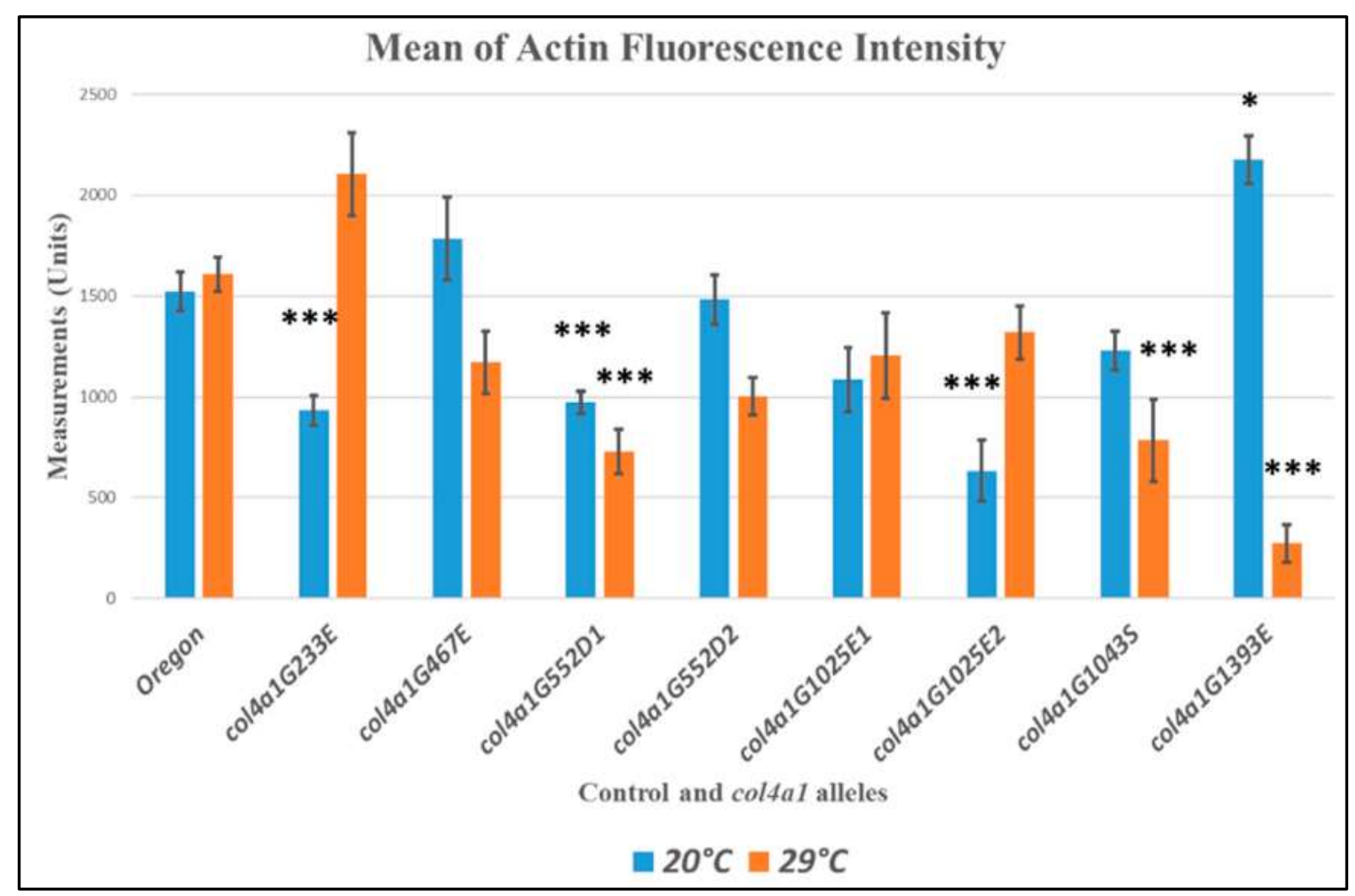

Figure 5. Quantitative measurements of actin content in muscle fibers by fluorescence light intensities of phalloidin labeled muscle. Significance is labelled by asterisk * $p<0.05 ; * * * p<$ 0.0005 .

The col4al ${ }^{G 233 E}$ mutant accumulates actin at $29{ }^{\circ} \mathrm{C}$, likewise to the col4a1 $1^{\text {G1025E2 }}$ mutant, whereas the actin concentration in the isoallelic col4al ${ }^{\text {G1025E1 }}$ mutant 
remains constant (Figure 4, panel C'). For the other mutants, we recorded higher actin concentration (Figure 4, panel C'). Up-regulation or down-regulation of actin expression in the common oviduct is unfavorable because all mutant females are sterile and do not lay eggs at the restrictive temperature ${ }^{40}$.

\section{Z-disc confusion, streaming and improper integrin expression in muscle tissue due to col4a1 mutations}

In wild-type Drosophila melanogaster striated muscle, integrin is expressed at the muscle attachment sites, showing dotted staining. This is noticeable in the case of Z-discs connected costameres ${ }^{61}$. To determine the exact position of the Z-discs in the fibers of the common oviduct muscle, we used an antibody against the protein called kettin, which is both a scaffold protein and a morphological marker. In wild-type controls, kettin staining appears in parallel lines in the muscle fibers perpendicular to the longitudinal axis. They always accurately represent the position of sarcomeres at both permissive and restrictive temperatures (Figure 4, A1, A4). Immunofluorescence using anti-integrin antibodies provided the same staining pattern in close localization as observed for kettin (Figure 6, A2, A5, and overlays (Figure 6, A3, A6), intensifying the correct integrin localization to the Z-discs in muscle fibers of the common oviduct.

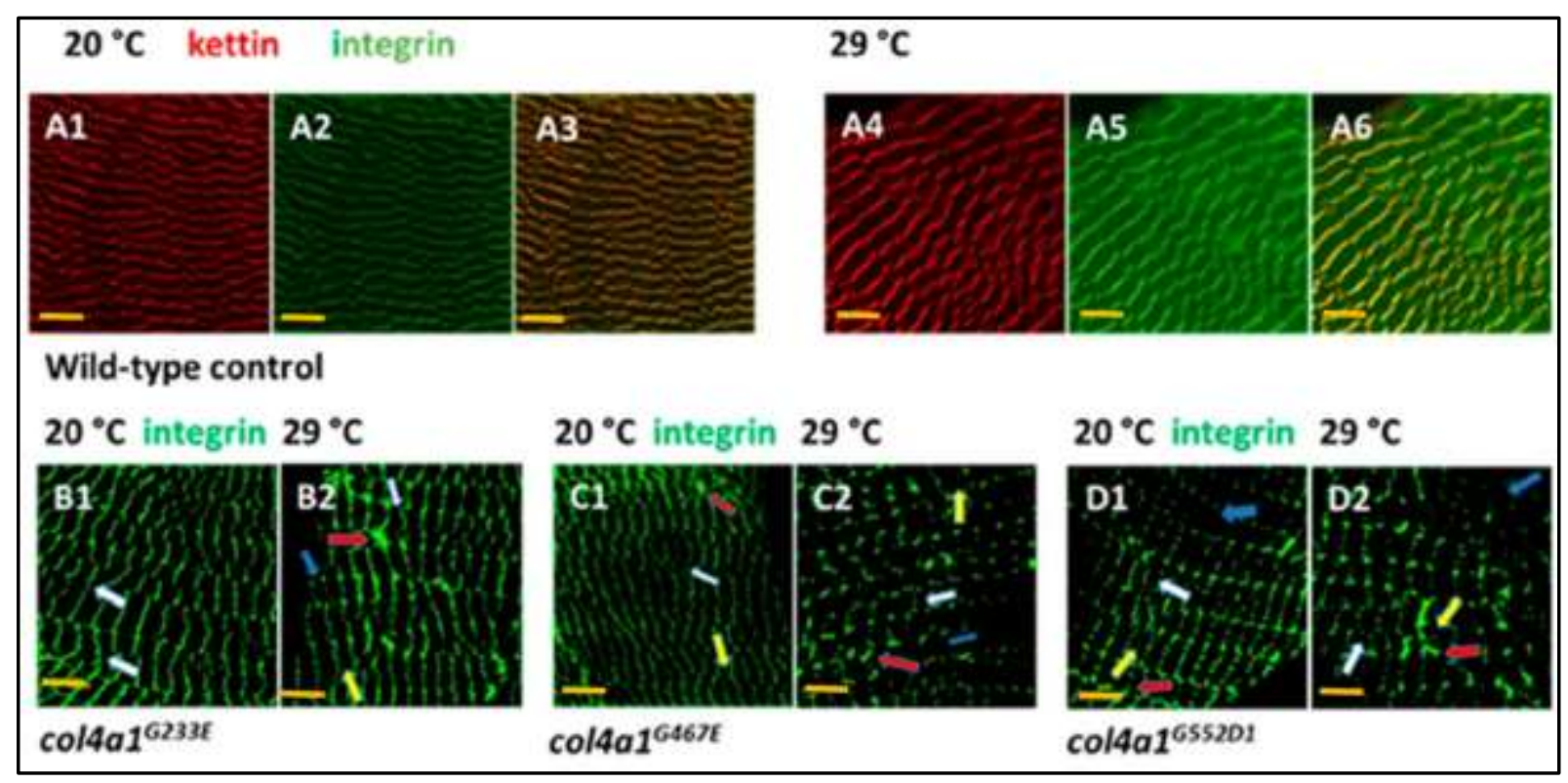


Figure 6. Staining of muscle fibers of common oviduct by the Z-disc marker kettin and integrin in wild-type control $(\mathbf{A 1}-\mathbf{A 6})$ at permissive and restrictive temperatures. Note unchanged integrin expression at $29^{\circ} \mathrm{C}$ (A5) and close vicinity of kettin and integrin depositions revealed by the complementary color orange in overlays (A3, A6). Panels $\boldsymbol{B}, \boldsymbol{C}, \boldsymbol{D}$ : col4a1 ${ }^{G 233 E}$, col4al ${ }^{G 467 E}$, col4al $^{G 552 D 1}$ mutants at permissive and restrictive temperatures. Streaming of the Z-discs (white arrows), integrin expression within the sarcomeres (yellow arrows), excess integrin expression (red arrows) or deficient integrin deposition (blue arrows) are noted in the mutants (panels $\boldsymbol{B}-\boldsymbol{D})$. Bars, lower left, 10 micrometers.

Aberrant integrin expression was observed in epithelial cells of Malpighian tubules of col4al mutants ${ }^{62}$, therefore, we assumed an irregular deposition of integrin in muscle fibers. In mutant common oviductal muscle fibers the Z-disc structure, delineated by integrin expression, was disrupted and formed a zig-zag pattern. Z-discs disintegrated in most of the sarcomere and showed a dotted pattern of integrin staining. Consistent with the muscle pathology of Z-disc streaming (Figure 6, white arrows, panels B-D), in which Z-disc structure is disrupted and Z-disc material seems splitted out and deposited within the sarcomere (Figure 6, yellow arrows, panels B-D). After incubation of the mutants at restrictive temperature, the following phenotypic characteristics were enhanced: Areas expressing excess integrin (Figure 6, panels B-D, red arrows), or depositing integrin barely (Figure 6, panels B-D, blue arrows). Z-disc streaming, intrasarcomeric integrin deposition and uneven distribution are characteristic features of col4al mutants, as these phenotypes have been observed in other members of the allele series.

Recently, additional phenotypic expression of mutants, ectopic assembly of Z-discs, and their transition from the anisotropic (A) band to the level of the M-discs have been reported, which is statistically significant in the mutants ${ }^{63}$. Taken together, these observations suggest that the appropriate COL4A1 protein is required for regular integrin expression, which, in the case of failure of the connection, results in female sterility, they do not lay eggs at $29{ }^{\circ} \mathrm{C}^{40}$. 


\section{Myopathic disorders related to col4al mutations in the common oviduct of Drosophila melanogaster}

Under restrictive temperatures, the female heterozygotes become progressively more female-sterile ${ }^{64}$. We choose the common oviduct as a model because it is surrounding the single layer muscle tissue. This model system allows us to directly examine the thin muscle tissue and the sarcoplasmic membrane without sectioning ${ }^{40}$. Muscle fibers in the col4a1 ${ }^{\text {G552D2 }}$ mutant detached laterally from each other and the thickened type IV collagen-associated label tried to fill the space. Muscle fibers covered by the basement membrane appear distorted, thinned, or missing, demonstrating that the mutant COL4A1 protein induced severe common oviductal myopathy ${ }^{40,65}$. We observed that at the Z-discs level of muscle fibers, the integrin deposition showed punctate staining in complete overlap with the signal of the kettin protein, which is a Z-disc marker. Each of our immunofluorescence preparations depicted the sacomere units ${ }^{66}$. High expressivity of the feature showed at low penetrance, i.e., shifting Z-disc integrin-binding sites toward the middle of sarcomeres in col4al $1^{G 552 D 1}$ mutants at the restrictive condition (Figure 7).

The regular integrin-kettin staining appears at the level of Z-discs, but we also get additional signals referring to duplicate Z-discs from the positions of the M-discs (Figure 7, B4, B5, B6, white arrows). In some parts of the numerous replications, the phenomenon is expressed with intense expressivity. Integrin staining quantitatively shifted to the level of the M-disc, the center of the anisotropic band (Figure 7, C1, C2, C3, white arrows). 


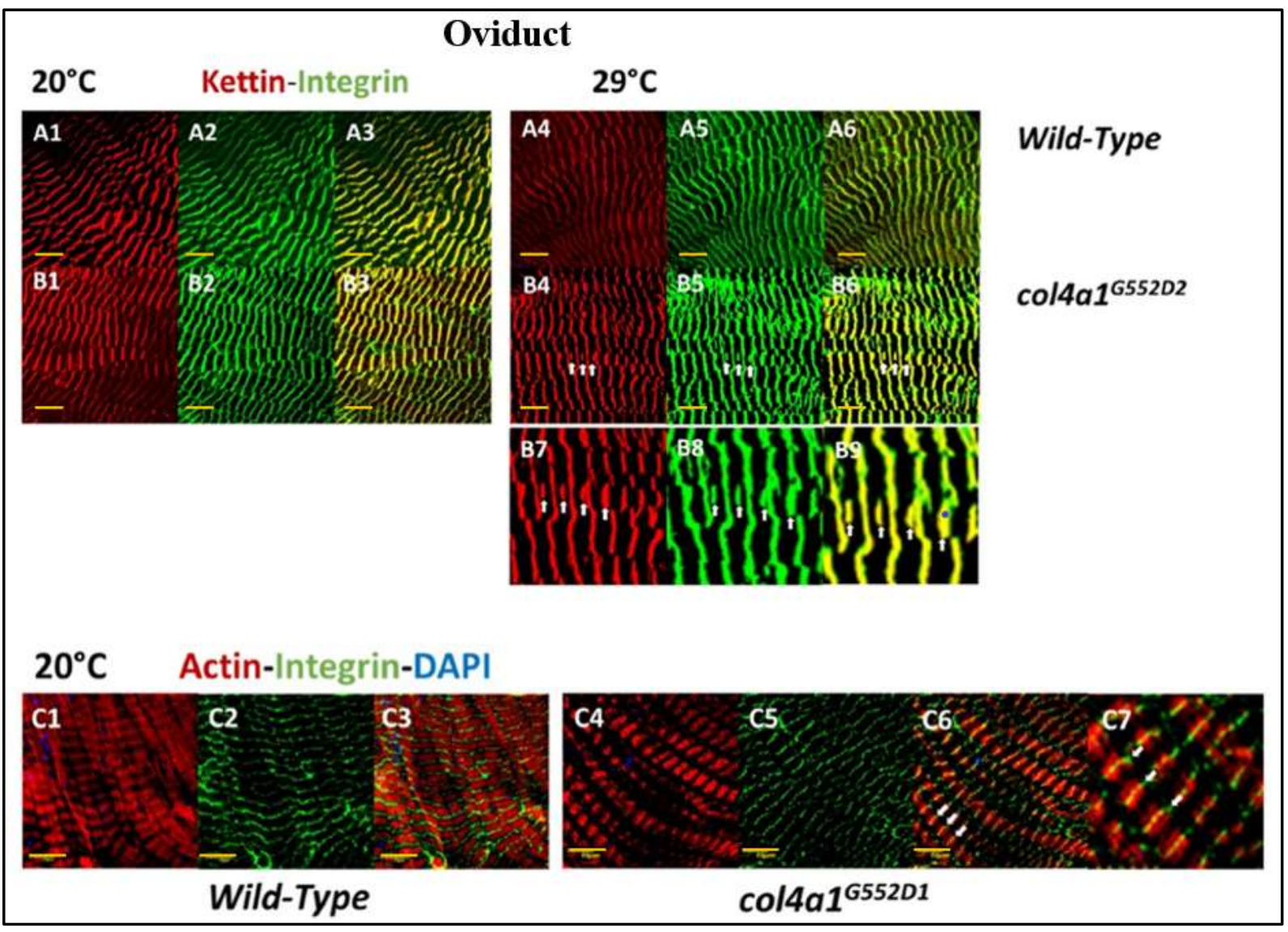

Figure 7. Shifting of Z-discs into the middle of sarcomeres, to the level of M-discs in striated muscle fibers of common oviduct. The scaffold protein kettin is deposited at the level of the Zdiscs $(A 1, A 4, B 1, B 4)$ and overlaps with the integrin label (A2, A5, B2, B5) providing yelloworange complementary colors in the overlays $(A 3, A 6, B 3, B 6)$. Note the irregular integrin expression in the mutant at $29^{\circ} \mathrm{C}$, zig-zag pattern and streaming of Z-discs (B5). Additional kettin-integrin expression appears in the middle of sarcomeres at the level of M-discs (white arrows, B4, B5, B6). Higher magnification of areas in B4, B5 and B6 are marked by arrows (B7, B8, B9). The shifting of the integrin expression toward the middle of the sarcomeres is nearly at full expressivity $(\mathrm{C4}, \mathrm{C5}, \mathrm{C6}$, white arrows). The area marked by arrows in C6 are at higher magnification (C7). Integrin expression remains at the sides of sarcomeres in Z-discs in the wild-type control (C1, C2, C3). Bars, lower left: 10 micrometers. Experiments were carried out as described ${ }^{40,65-68}$. 
The Z-disc shift phenomenon was then analyzed quantitatively in wild-type controls (Oregon) and col4a1 ${ }^{G 552 D 1}$ mutants. The level of expressivity is indicated by the Z-disc marker kettin antibody (Figure 8). The expressivity of the Z-disc shift is higher than $8 \%$ in wild-type controls and greater than $12 \%$ in col $_{4 a 1} 1^{\text {G52D2 }}$ mutants (Figure 8 ). The results were obtained by counting 6796 kettin-stained structures in the single-layer striated muscle of the common oviduct. Statistical analysis was performed using Welch's two-sample t-test. The p-value of significance was 0.003973 , indicating a powerful expression of the Z-disc shift in the mutants (Figure 8).

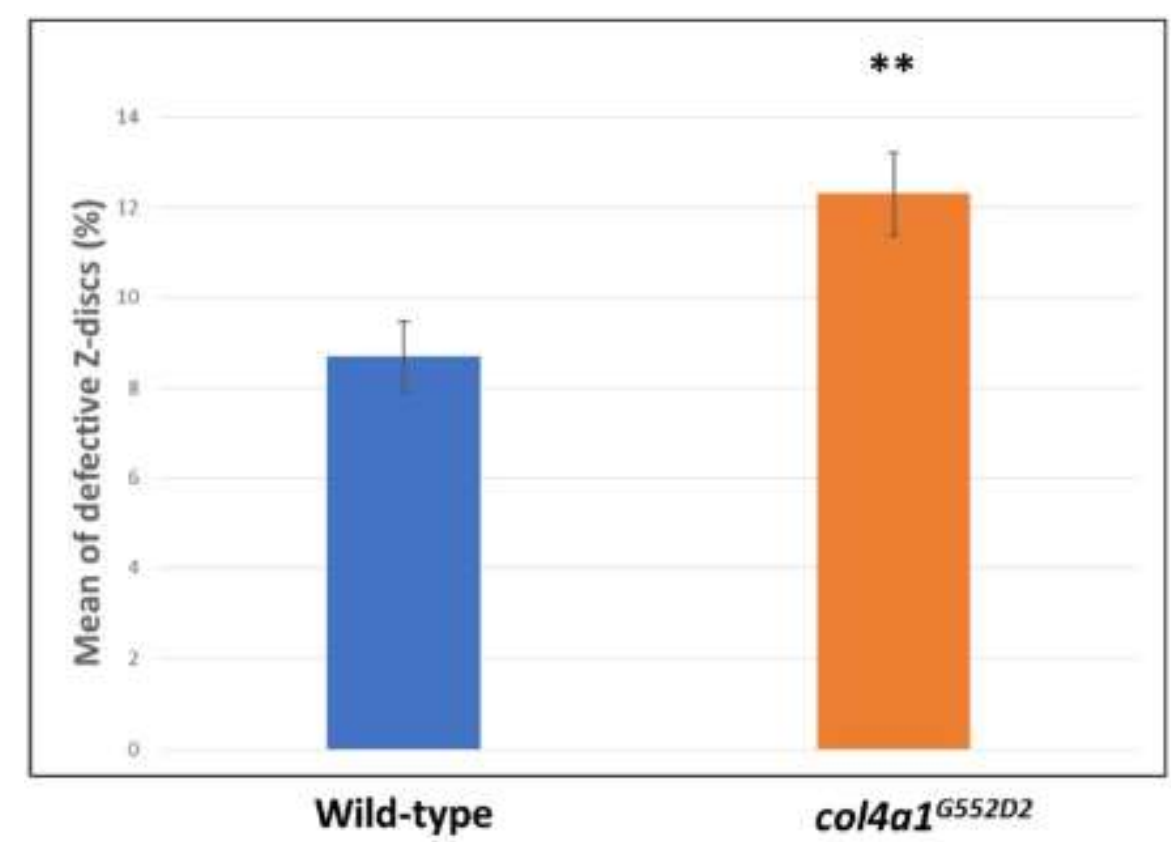

Figure 8. Elevated expressivity of Z-disc shifting in mutants. Statistically significant expressivity of Z-disc shifting in the mutants at $p=0.003973(* *)$.

\section{Structural changes in the basal membrane components of the midgut in col4a1 mutants}

We analyzed the expression and distribution of BM components, such as the PSI and PSII alpha subunits of integrin, laminin gamma 1, and collagen COL4A1 in the midgut by immunofluorescence (Figure 9). Panels A1-A3 present the visceral muscle cells of the midgut 
in control L3 larvae, A4-6 correspond to mutant midgut stained for integrins (green) and actin (red) at $20^{\circ} \mathrm{C}$. Punctated integrin staining is seen in the control larvae, which is localized to the Z-discs, but slightly less integrin expression was detected in the same location in the mutants. This phenomenon became even more evident in those larvae were incubated at $29^{\circ} \mathrm{C}$, and large areas in the midgut of the mutant lacked integrin expression. (asterisks, Figure. 9, B1-3 vs B46). The same integrin expression pattern was observed in the midgut of adult mutant animals (Figure 10). Similarly, levels of Laminin gamma 1 were reduced in the midgut of L3 larvae (Figure 9, C3 vs C4, red) and in the midgut of adult animals (Figure 9, C7 vs C8, red) at $29^{\circ} \mathrm{C}$ and appeared as diffuse staining in mutants $(\mathrm{C} 4, \mathrm{C} 8)$. COL4A1 staining concentrated in the sarcoplasm in the midgut of the mutant at $20^{\circ} \mathrm{C}$ (Figure 9, D3, D4, green) and indicated a diffuse, irregular staining pattern at $29^{\circ} \mathrm{C}$ (Figure 9, D7, D8, green, asterisks). These results confirmed our earlier data and corroborated a systemic, multi-organ phenotype in col4al mutants at the restrictive temperature ${ }^{32,65}$.

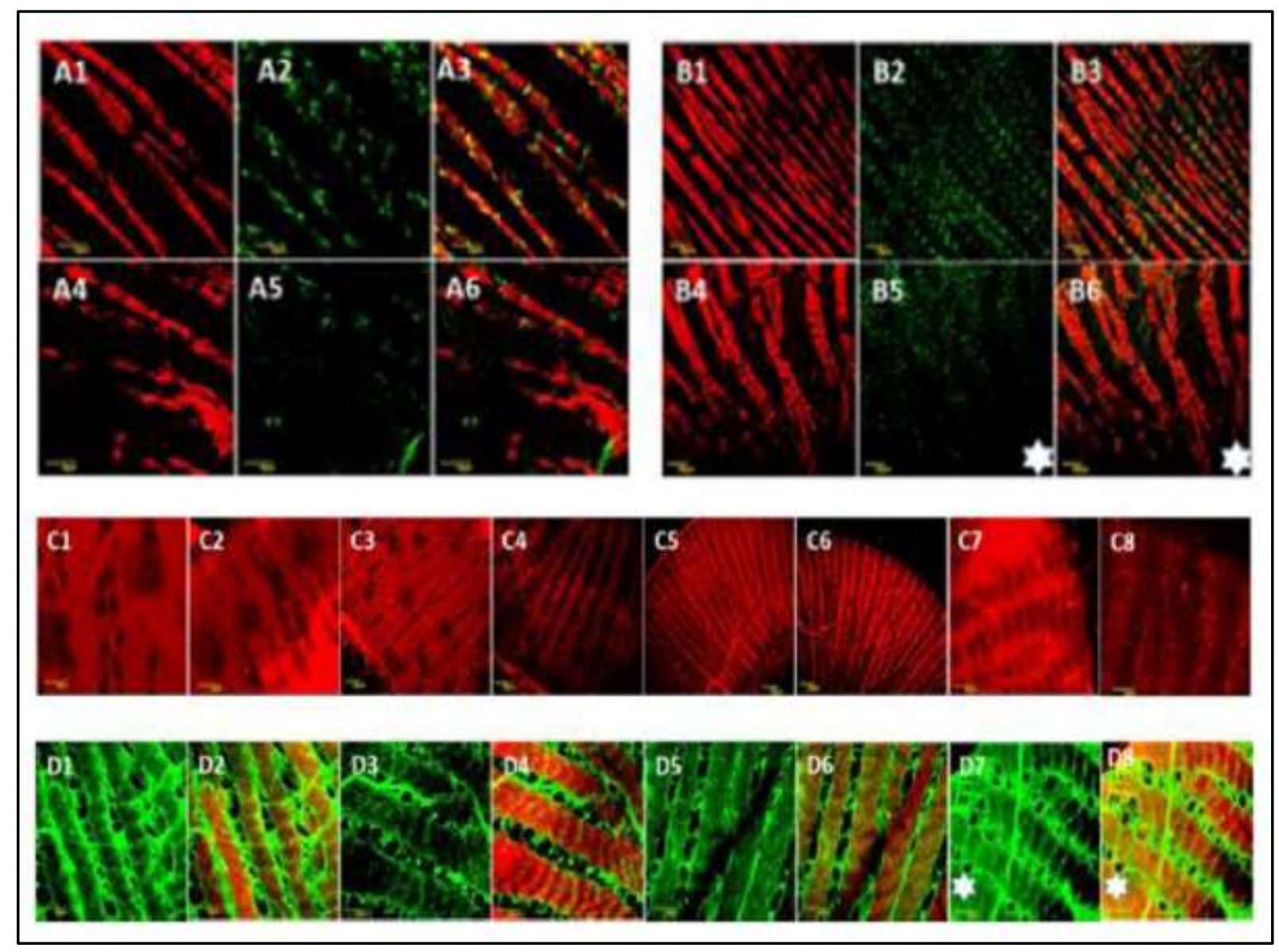


Figure 9. Structural proteins disruption in the midgut of control and mutant larvae (A1-C8) and adults (D1-D8). Actin (red) and integrin (green) staining of the L3 larval midgut visceral muscle cells. A1: Actin, A2: Integrin, A3: Merge, wild-type; A4-6, mutant, same order, $20^{\circ} \mathrm{C}$. B1-3, wild-type, B4-6 mutant, $29^{\circ} \mathrm{C}$. Asterisks refer to areas of lower integrin expression. C1, C2: Laminin expression (red) in the midgut of L3 control/mutant larvae at $20^{\circ} \mathrm{C}, \mathrm{C} 3, \mathrm{C4}$, at 29 ${ }^{\circ} \mathrm{C}$. C5, C6: Laminin expression in the midgut of control/mutant adults, $20^{\circ} \mathrm{C}, \mathrm{C7}, \mathrm{C8}, 29^{\circ} \mathrm{C}$. Note decreased laminin expression in C4 and C8. COL4A1 staining in wild-type at $20^{\circ} \mathrm{C}(\mathrm{D} 1)$, and at $29{ }^{\circ} \mathrm{C}(\mathrm{D} 5)$, mutant at $20{ }^{\circ} \mathrm{C}(\mathrm{D} 3)$, and mutant at $29{ }^{\circ} \mathrm{C}$ (D7). COL4A1 staining concentrated in the sarcoplasm in the midgut of the mutant at $20^{\circ} \mathrm{C}(\mathrm{D} 3, \mathrm{D} 4$, green) and indicated a diffuse, atypical staining pattern at $29{ }^{\circ} \mathrm{C}$ (D7, D8, asterisks). Merge with actin staining (D2, D4, D6, D8).

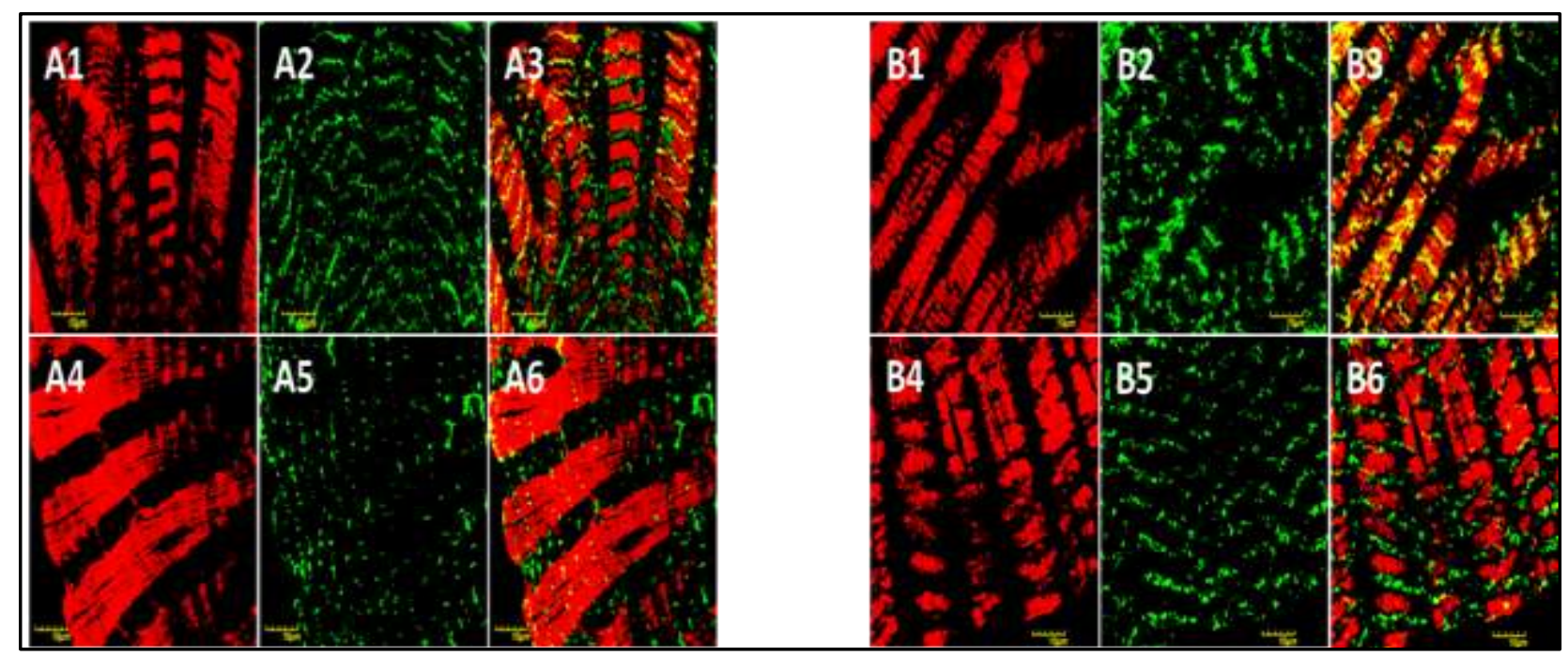

Figure 10. Staining of actin (red) and integrin PSI and PSII alpha subunits (green) in the midgut of adult animals. A1: Actin, A2: integrin, A3: Merge in wild-type controls, $20{ }^{\circ} \mathrm{C}$; A4A6, mutant, $20^{\circ} \mathrm{C}$. B1-B3: Wild-type controls, B4-B6 mutant, $29^{\circ} \mathrm{C}$, all in the same order. Note reduced and irregular integrin staining in the midgut of col4al mutant flies. Scale bar: $10 \mu \mathrm{m}$.

The basic cellular functions regulated by the microenvironment are provided by the three-dimensional structure of the ECM and its components. Cell survival and immune system activation are also affected by ECM structure and aberrant expression of its elements, which can induce pathological processes and tissue remodeling. Degraded fragments of the BM also modulate the activities of both resident cells and infiltrating immune cells ${ }^{70}$. Type IV 
collagen fragmentation during the BM degradation by metalloproteinases were implicated in the synthesis of antimicrobial peptides (AMPs) and stimulation of innate immune responses during insect metamorphosis ${ }^{71}$. Endogenously derived danger/alarm signals, including type IV collagen fragments, also stimulated immune cells in lepidoptera ${ }^{72}$. Modulated genetic elements of the BM components (laminin, nidogen/entactin, perlecan, integrins, and cross-linking lysyl oxidases) are available in Drosophila ${ }^{73,74,75}$, the specific role of aberrant BM and the immune modulatory function of type IV collagen have not yet been investigated in this model.

We have previously demonstrated that temperature-sensitive col4al Drosophila mutants have altered BM structure and function, resulting in severe myopathy leading to degradation of skeletal and visceral muscle cells ${ }^{40,65}$. The results of our current studies confirmed that mutated col4al could also cause degradation of epithelial cells in the gut at restrictive temperatures. After the continuous incubation of the mutants at $29{ }^{\circ} \mathrm{C}$, they range from egg-laying to manifesting morphological changes such as shorter larvae, the development of severe gut pathology, including the shorter gut, enlarged midgut, multiple diverticulae, lead to intestinal dysfunction and shorter life span.

Based on these data, the question arises as why chronic inflammation does not occur in mice and human patients bearing the COLAAl mutation due to the high activity of the immune system? The Drosophila col4al mutation differs in many aspects from the genetic modification of mammals. Homologous Drosophila mutations have no effect on viability, fertility, and do not cause detectable BM-associated cell degradation at the permissive temperature. It is only when the temperature is severe that the pathological systemic phenotype occurs. A similar stress-induced phenotype was also observed in $\mathrm{Col}_{4 a 1} \mathrm{~T}^{-/}$mouse embryos. Col4al or Col4a2 null homozygotes were lethal during the developmental phase when the embryonic heart began to beat, and the blood vessels were pressurized ${ }^{46}$.

Our results with the Drosophila col4al conditional dominant mutant line support the applicability of this model to investigate the mechanical elements of human COL4-associated conditions. Mutant phenotypic characteristics confirm that collagen type IV plays an essential role in maintaining epithelial integration, gut morphology, and intestinal function. Furthermore, 
it suggests that the aberrant structure and function of the COL4A1 protein may be a significant factor in the modulation of the immune system.

\section{Disorder of the excretory system of Drosophila melanogaster col4a1 mutants}

Metabolism produces numerous harmful and redundant substances that are carried out by the kidneys in mammals and by the Malpighian tubules in insects. As part of the clinical spectrum, amino acid substitution in some ubiquitous BM components, such as COL4A1, may cause nephropathy. These changes lead to renal dysfunction and chronic renal failure in the affected patients, with or without hematuria. In mouse Col4a1 mutants, the same symptoms were observed as in humans. The mammalian kidney is functionally identical to the Malpighian tubule and therefore provides a versatile and tractable model for it is examination ${ }^{76}$. Freefloating tubules are in constant movement within the hemocoel, which is an insect blood-filled body cavity with an open circulatory system. Mechanical stress of intermittent motility utilizes and contributes to stress-induced cytoskeletal rearrangement in col4al mutant animals. Therefore, we investigated the effects of mechanical loading on the col4al $1^{\text {G52D2 }}$ mutant and noted the formation of actin stress fibers and irregular, uneven actin staining in epithelial cells of the malpighian tubule ${ }^{62}$. In another study of ours, we observed the mitochondrial fusion and co-localization of peroxynitrite-nitrated and 4-hydroxy-2-nonenal alkylated proteins with abnormal mitochondria ${ }^{68}$. Mitochondrial fusion in the Malpighian epithelium is expressed in the col4al ${ }^{G 552 D 1}$ mutant at the restrictive temperature (Figure 11), as a sign of stress induced by the mutation. At the same time, the qualitatively estimated level of protein nitration remains unchanged in wild-type control and mutant strains under both permissive and restrictive conditions (Figure 11). 


\section{Malpighian tubule}

$20^{\circ} \mathrm{C}$ Actin-Mitochondria-Nitrothyrosine $29^{\circ} \mathrm{C}$

Wild-Type
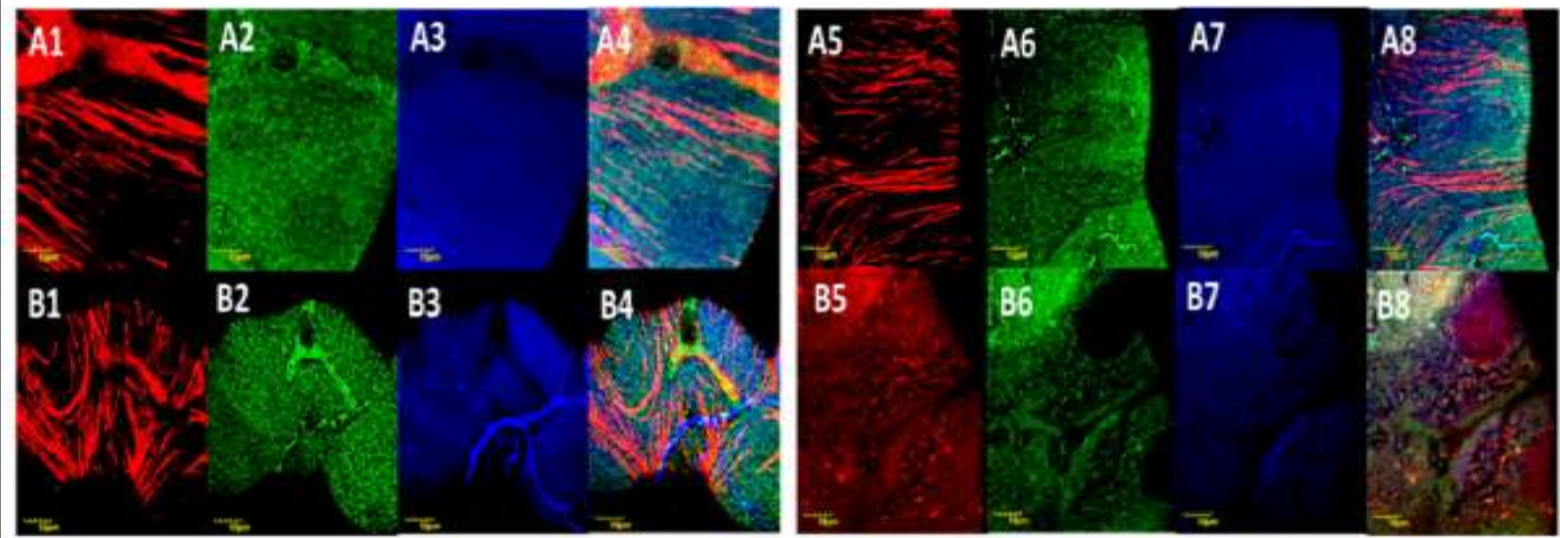

col4a1 ${ }^{655201}$

Figure 11. Compromised Malpighian tubules in mutants. Fusion and uneven distribution of mitochondria as a consequence of mutation-induced stress. Even distribution of mitochondria in wild-type control $(\mathbf{A 2}, \boldsymbol{A 4})$ and in the mutant under the permissive condition $(\mathbf{B 2}, \mathbf{B 4})$. The normal distribution of mitochondria remained at restrictive condition (A6, A8). Large aggregates of mitochondria appear in the mutant at $29^{\circ} \mathrm{C}(\boldsymbol{B 6}, \mathbf{B 8})$. Actin staining (A1, A5, B1, B5). Nitrothyrosine staining (A3, A7, B3, B7). Bars, lower left: 10 micrometers.

\section{Changes at the expression level of actin, COLAA1 and integrin in} the Malphigian tubules

Dominant temperature-sensitive mutations were identified in the coltal gene of Drosophila, being the insect homolog to the mammalian COL4A1 gene. Like their mammalian counterparts, they mutations induce a systemic phenotype including severe myopathy, intestinal dysfunction, and a robust immune response through overexpression of antimicrobial peptides and overexpression of oxidants, manifestly in the form of hydrogen peroxide and peroxynitrite 32,44 . Functionally similar to the mammalian kidneys, the insect excretory organs are the 
Malpighian tubules ${ }^{76}$. The tubules float freely within the hemocoel, in the open circulatory system filled with blood in the cavity of the body. It was hypothesized that the mechanical impulse of the periodic movement of the insect body would keep the Malpighian tubules in continuous motion, which may contribute to the stress-induced cytoskeletal reorganization of mutant animals.

The DTS L3 allele was selected from the mutant lines to determine the phenotype of the Malpighian tubules associated with the mutant Drosophila col4al gene ${ }^{32,38,44}$, as a model, and compared to wild-type Oregon $R$ flies. Both lines were incubated at permissive $\left(20{ }^{\circ} \mathrm{C}\right)$ and restrictive $\left(29^{\circ} \mathrm{C}\right)$ temperatures and evaluated following three and eighteen days of incubation. In both wild-type and mutant flies, actomyosin accumulated in the cortical periphery of epithelial cells, irrespective of incubation time at the permissive temperature, whereas COL4A1 protein staining presented a regular distribution (Figure 12). The epithelial cells of the col4al mutant developed actin stress fibrils, which were visible after 3 days of incubation at restrictive temperatures (Figure 13, A4, white arrow), which became more abundant in the cytoplasm after 18 days of incubation (Figure 13, A5). The results obtained were similar to those reported for cytoskeletal rearrangement of the proximal tubules of the kidneys of Col4a1 ${ }^{\mathrm{G} 498 \mathrm{~V} / \mathrm{G} 498 \mathrm{~V}}$ mutant homozygous mice ${ }^{77}$. In the mutants, epithelial cells also expressed less COL4A1 protein both at the third (Figure 13, A5, yellow arrows) and eighteenth day (Figure 13, B5, red arrows) of incubation at $29^{\circ} \mathrm{C}$.

Type IV collagens, as ligands, bind with high affinity to integrin, which are BM cell surface receptors and this construct is part of the cytoskeletal-extracellular matrix axis ${ }^{78}$. Dynamic interactions between ECM / BM and the actin cytoskeleton are mediated by trans membrane integrins, including mechano-transduction.

To evaluate the changes in BM-cytoskeletal effects caused by the altered type IV collagen network in mutant animals, we determined the distribution and expression of the PSI alpha and PSII alpha subunits of the integrin. Evenly distributed integrin was detected immunofluorescently, which showed punctate staining on the surface of epithelial cells of the Malpighian tubules (Figure 14) and integrin alpha subunits also co-localized with actin staining as demonstrated by green (integrin) and red (actin) labeling in the merged photomicrographs (Figure 14, A3, A6, B3, B6, orange) in both mutant and wild-type lines at permissive 
temperature irrespective of the age of the flies or length of incubation. Mutant lines developed actin stress fibers by day 3 at elevated temperature (Figure 15, A4, white arrow) that persisted at day 18 (Figure 15, B4, white arrows), a feature that was not observed in wild-type animals (Figure 15, A1, B1). Integrin staining was uneven with areas of minimal expression at day 18 in mutants (Figure 15, B5, yellow arrows). Additionally, integrin and actin connections appeared interrupted as these proteins were no longer co-localized, demonstrated by areas with isolated integrin staining (Figure 15, B6, red arrows). Integrin and actin were in close proximity to each other in wild-type control, thus supporting the existence of a cytoskeleton-ECM linkage (Figure 15, B3). Taken together, these data demonstrate that the expression of integrin is affected by the col4al mutation, induces abnormal integrin accumulation, and increases the number of stress fibrils in epithelial cells of the Malpighian tubules due to impaired mechanotransduction. In the Drosophila col4al mutant model, these phenotypic changes represent cellular markers suitable for the rapid and cost-effective evaluation of targeted therapeutic interventions.

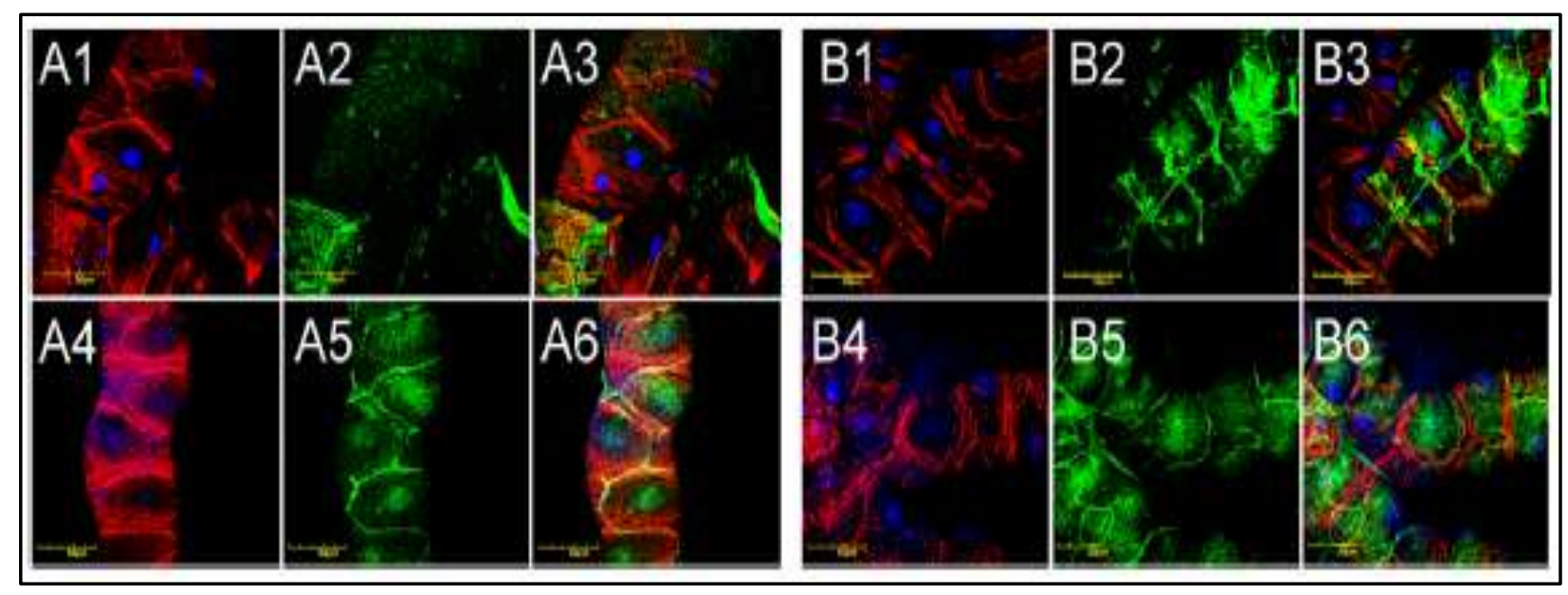

Figure 12. Malpighian tubules of wild-type (A1-A3 and B1-B3) and mutant (A4-A6 and B4B6) flies kept at $20{ }^{\circ} \mathrm{C}$ for 3 and 18 days. Red: Actin, blue: DAPI-stained nuclei, green: COLAA1. Bars equal $50 \mu \mathrm{m}$. 


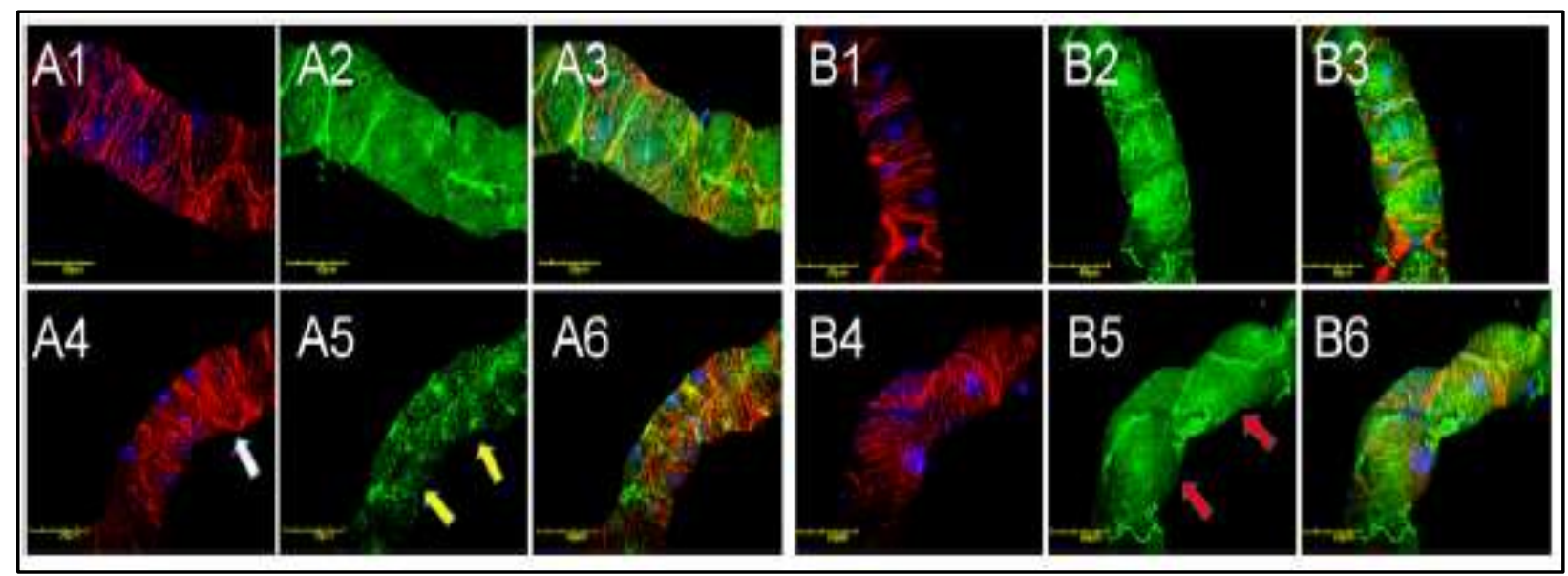

Figure 13. COLAAI and stress fiber detection in Malpighian tubules of wild-type (A1-A3 and B1-B3) and mutant (A4-A6 and B4-B6) flies incubated at $29^{\circ} \mathrm{C}$ for 3 and 18 days. Red: Actin, blue: DAPI-stained nuclei, green: COLAA1. Stress fibers appeared in mutants following three days incubation at $29^{\circ} \mathrm{C}$ (A4, white arrow) and became more abundant by day 18 (B4); mutant Malpighian tubules express less COL4A1 (A5, yellow arrows, B5, red arrows). Bars equal 50 $\mu m$.

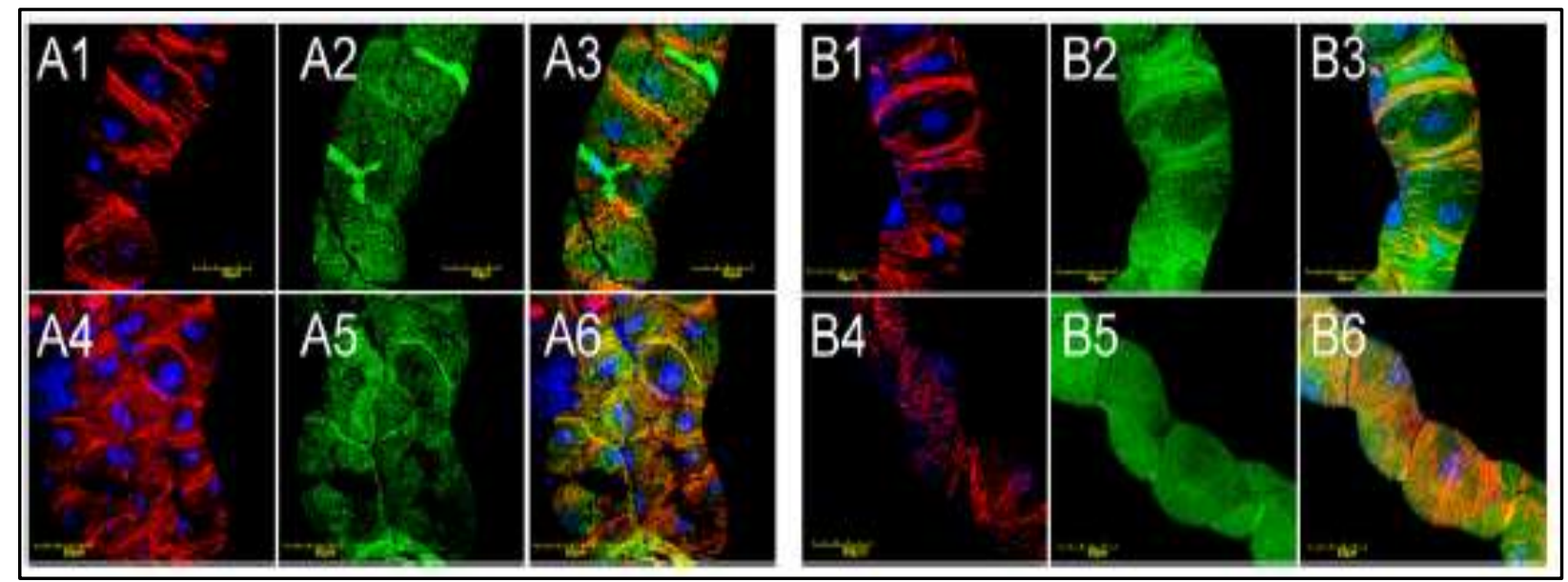

Figure 14. Integrin detection in Malpighian tubules of wild-type (A1-A3 and B1-B3) and mutant (A4-A6 and B4-B6) flies incubated at $20^{\circ} \mathrm{C}$ for 3 and 18 days. Red: Actin, blue: DAPIstained nuclei, green: Integrin-staining by anti-PSI alpha and PSII alpha antibodies. Bars equal $50 \mu m$. 


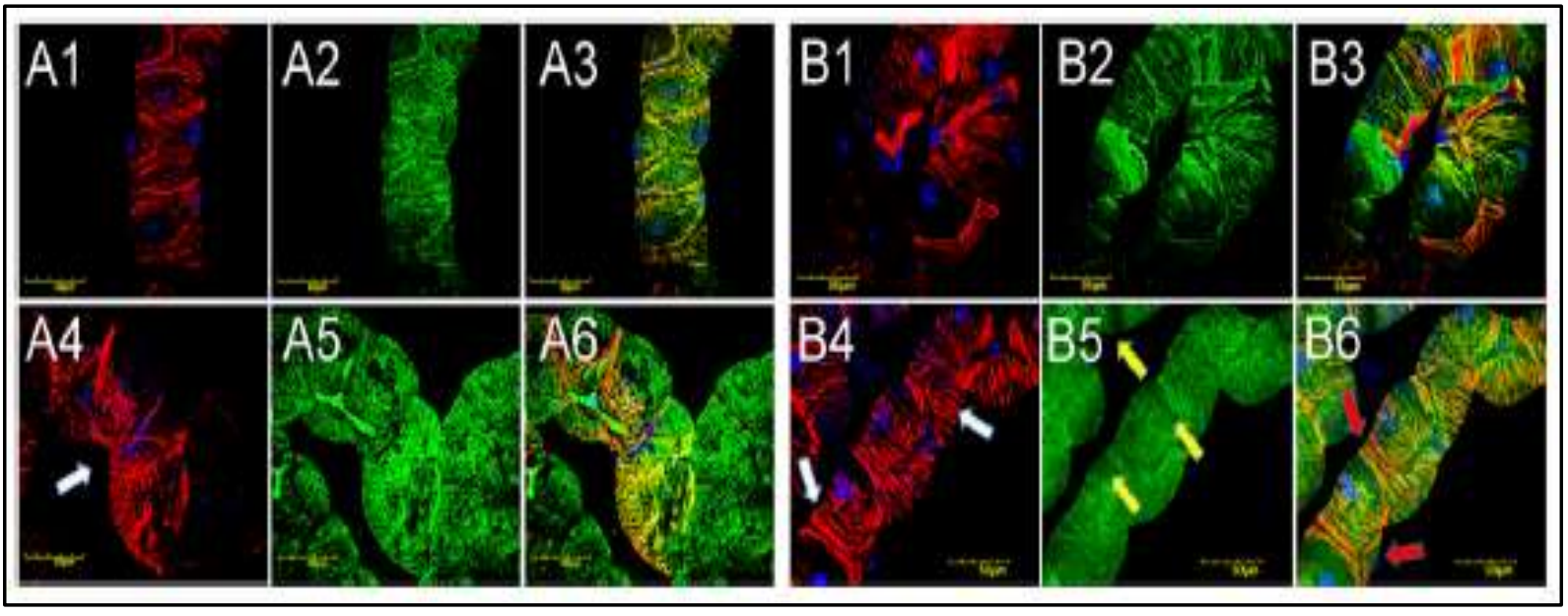

Figure 15. Altered integrin localization and stress fibers in Malpighian tubules of wild-type (A1-A3 and B1-B3) and mutant (A4-A6 and B4-B6) flies incubated at $29^{\circ} \mathrm{C}$ for 3 and 18 days. Red: Actin, blue: DAPI-stained nuclei, green: Integrin-staining by anti-PSI alpha and PSII alpha antibodies. Stress fibers appeared following three days of heat shock (A4, whitearrow) and became more abundant by day 18 (B4, white arrows). Alpha integrins co-localized with actin (B3) in wild-type controls whereas in mutants, large areas showed reduced staining for integrins (B5, yellow arrow), furthermore, integrin signals did not co-localize with actin (B6, red arrows). Bars equal $50 \mu \mathrm{m}$.

\section{Elevated peroxynitrite levels and mitochondrial fusion in mutant}

\section{Malpighian tubules}

We have previously demonstrated that, under restrictive conditions, col4al mutant flies synthesize peroxynitrite at higher concentrations as part of their antimicrobial immune response 67. Peroxynitrite is produced by a diffusion-driven reaction between nitric oxide (NO) and oxidants such as the radical superoxide anion $\left(\mathrm{O}^{*}{ }^{\circ}\right)$ derived from mitochondria. Sources of NO come from the extramitochondrial side, and the dissolved gas diffuses into the mitochondria. It reacts with $\mathrm{O}_{2}{ }^{-}$inside the mitochondria and interrupts the protein functions by nitrating the tyrosines of the proteins ${ }^{79}$. Therefore, it was expected that nitrated proteins would accumulate in the mitochondria of col4al mutant flies following incubation at $29^{\circ} \mathrm{C}$.

No major changes were observed in the Malpighian tubules of the mutants compared to control flies. The mitochondria distributed evenly in the cytoplasm, and the fluorescent light 
intensity of the labeled nitrated proteins from the organs of the mutant animals was comparable to the control animals (Figure 16, a-c) and (Figure 16, g-i) following incubation at the permissive temperature. However, under restrictive conditions $\left(29^{\circ} \mathrm{C}\right)$, observable differences were inspected in the Malpighian tubules of the mutant flies. While mitochondria in the epithelial cells of wild-type Malpighian tubules remained evenly distributed with no shape alteration at this temperature (Figure 16, d), in mutants, uneven distribution and mitochondrial fusion were observed (Figure 16, j). The level of nitrated proteins was remarkably higher in mutants in comparison with the control (Figure 16, k vs e) and these signals localized to the mitochondria (Figure 16, f, l).

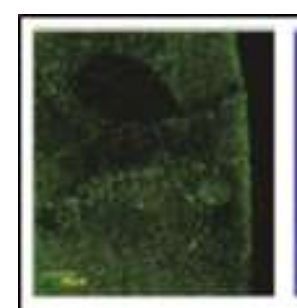

(a)

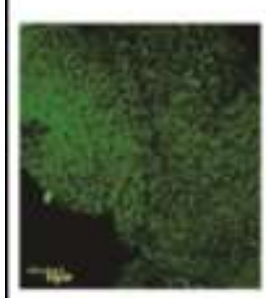

(g)

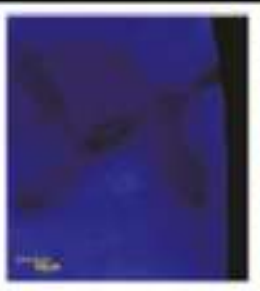

(b)

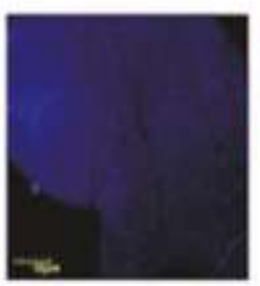

(h)

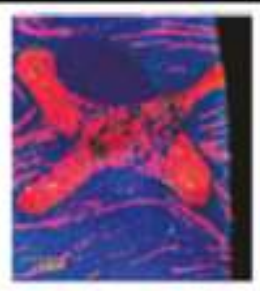

(c)

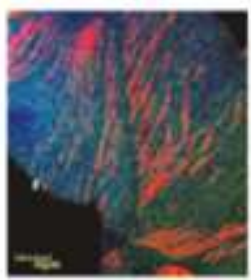

(i)

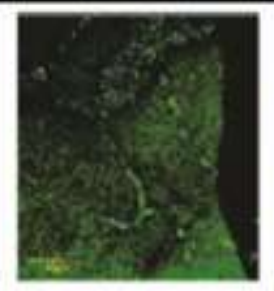

(d)

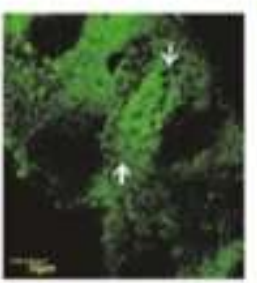

(i)

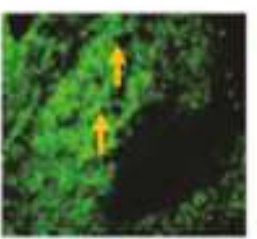

(m)

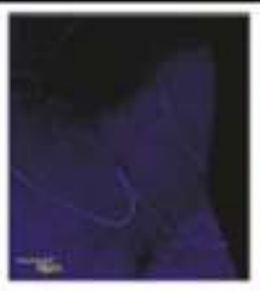

(e)

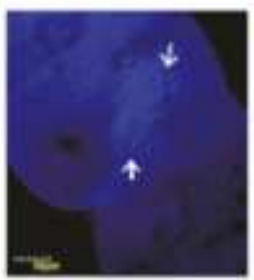

(k)

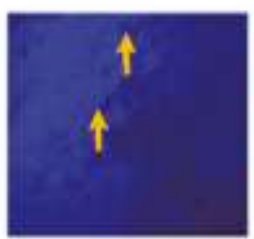

(n)

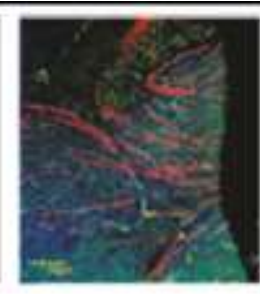

(f)

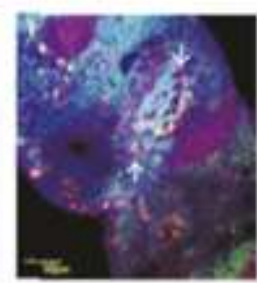

(1)

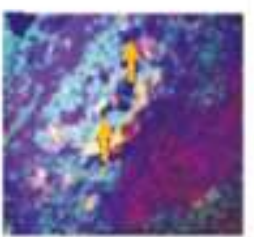

(o)

Figure 16. Protein nitration in Malpighian epithelial cells. Color code: mitochondria, green; nitrated proteins, blue; actin, red. (a) Wild-type flies incubated at $20^{\circ} \mathrm{C}$, mitochondria, (b) nitrated proteins, and (c)merge. Note a stellate cell in $(c) .(d),(e),(f)$ Wild-type flies incubated at $29^{\circ} \mathrm{C}$ and displayed in the same order. ( $\mathrm{g}$ )-(i) Mutant flies, incubated at $20^{\circ} \mathrm{C}$, and $(\mathrm{j})-(\mathrm{l})$ mutant flies, incubated at $29^{\circ} \mathrm{C}$. Photomicrographs are displayed in the same order as in the 
upper row. Localization of nitrated proteins to mitochondria is shown in (c), $(i),(f)$, and $(l)$. Uneven distribution and fusion of mitochondria are demonstrated in $(j)$. White arrows in $(j)$, $(k)$, and $(l)$ define the regions displayed in higher magnification in $(m),(n)$, and $(o)$. Yellow arrows in (m), (n), and (o) point to areas with nolfew mitochondria and the lack of staining, demonstrating colocalisation of mitochondria with nitrated proteins indirectly.

\section{Elevated levels of alkylated proteins and uneven distribution of mitochondria in the col4a1 mutants Malpighian tubules}

The level of lipid peroxidation was determined indirectly by the accumulation of HNEprotein adducts. Under permissive conditions, epithelial cells of wild type and mutant Malpighian tubules yielded comparable amounts of alkylated protein (Figure 17, b vs h), and the mutation had effect in the appearance of mitochondria in this condition (Figure 17, a vs g). Under restrictive conditions $\left(29^{\circ} \mathrm{C}\right)$, uneven distribution and fusion of mitochondria can be observed in mutants (Figure 17, j vs d), the mutants produced more HNE-protein adducts (Figure 17, k vs e), and the alkylated proteins localized to mitochondria (Figure 17, f, 1).

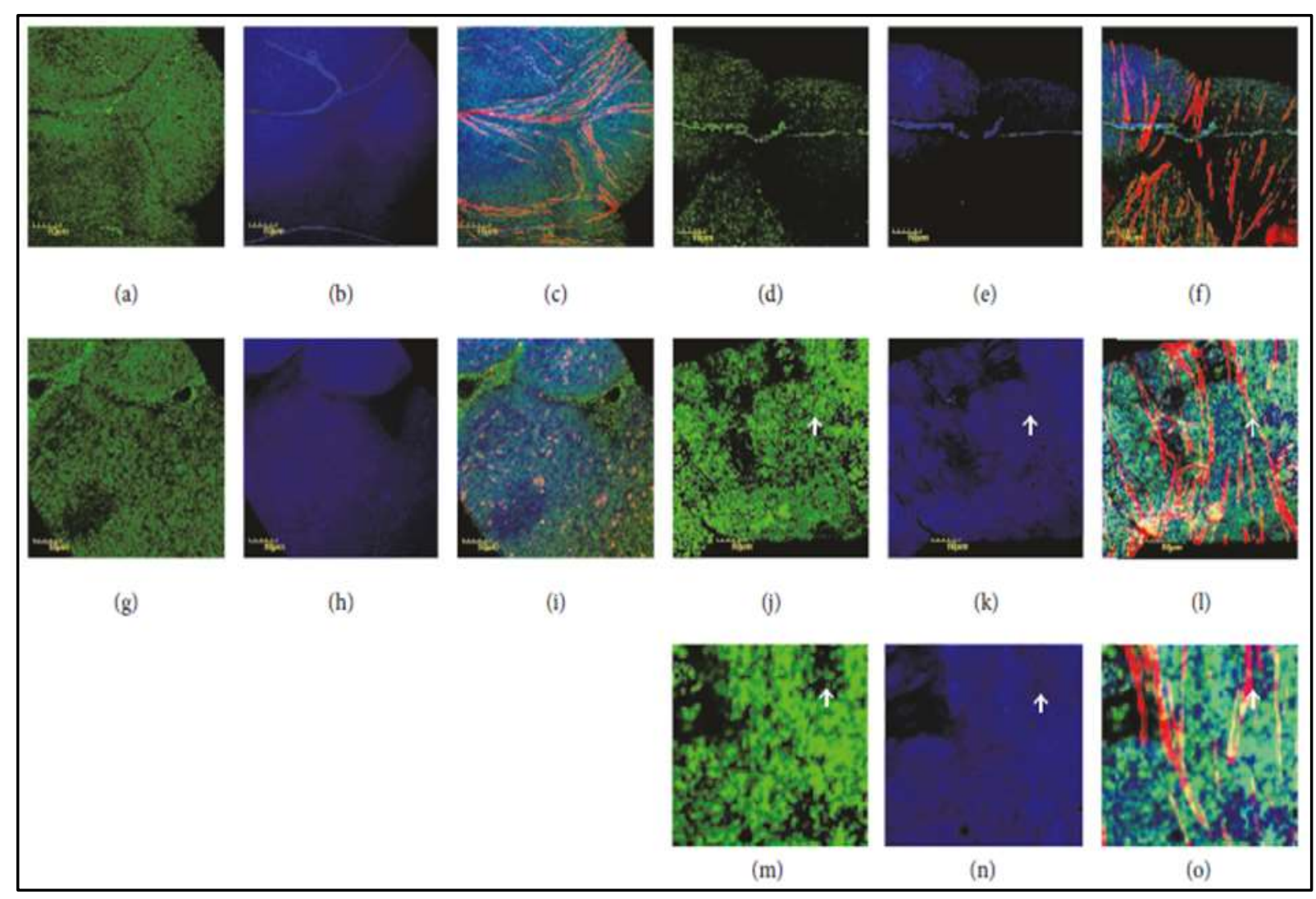


Figure 17. Protein-HNE adducts in Malpighian epithelium. Color code: mitochondria, green; protein-HNE adducts, blue; actin, red. (a) Mitochondria of wild-type flies incubated at $20^{\circ} \mathrm{C}$, (b) protein-HNE adducts, and (c) merge. (d), (e), (f). Wild-type flies incubated at $29^{\circ} \mathrm{C}$, shown in the same order. (g)-(i) Mutant flies, incubated at $20^{\circ} \mathrm{C}$, and (j)-(l) mutant flies, incubated at $29^{\circ}$ C.The order of photomicrographs is as in upper row. Note mitochondrial fusion in (j) and actin stress fibers in $(l)$. The white arrow in $(j),(k)$, and $(l)$ showing the portion displayed in higher magnification in $(m),(n)$, and $(o)$. The white arrow in $(m),(n)$, and $(o)$ : point regions with no/few mitochondria and the lack of staining, demonstrating localization of alkylated proteins to mitochondria indirectly.

\section{Lipid peroxidation in the cytoplasmatic membrane of mutant}

\section{Malpighian tubules}

We then examined the role of the cytoplasmic membrane in col4al-associated pathology. Numerous alkylation sites were observed in the form of punctate staining in colocalization with the cytoplasmic side of the membrane, and apparent perinuclear accumulation in mutant Malpighian epithelial cells under permissive conditions. $\left(20{ }^{\circ} \mathrm{C}\right)$ (Figure 18, e, f). This staining pattern was amplified to raise to the restrictive temperature (29 ${ }^{\circ} \mathrm{C}$ ), and HNE conjugated proteins appeared in the cytoplasmic membrane, indicating direct membrane damage by lipid peroxidation (Figure 18, g, h). In the control flies the cytoplasmic membrane remained intact, and protein-HNE adducts appeared in the vicinity of the membrane at both permissive and restrictive conditions (Figure 18, a-d). 


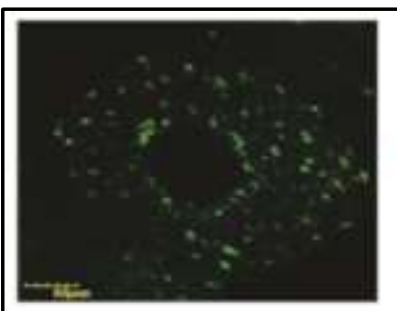

(a)

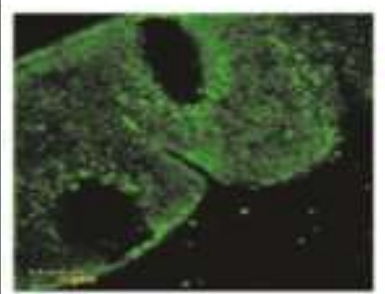

(e)

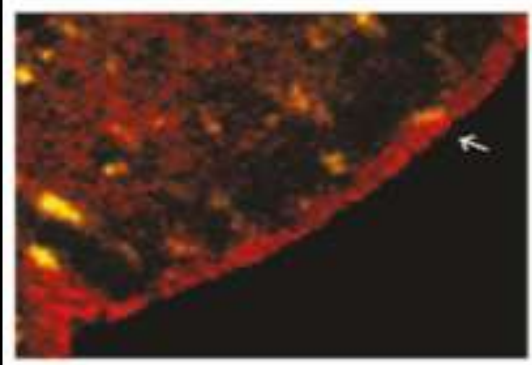

(i)

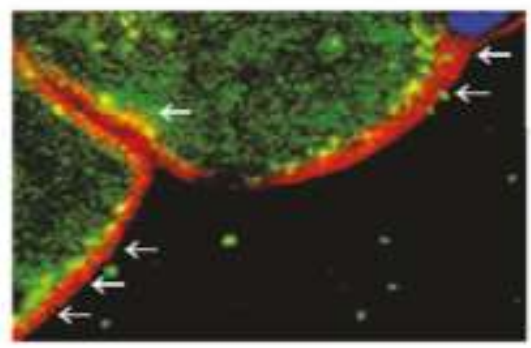

(1)

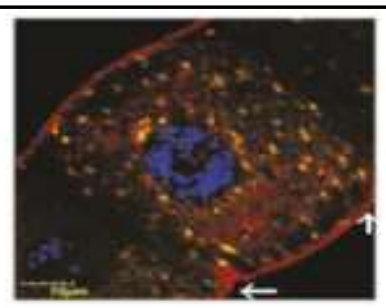

(b)

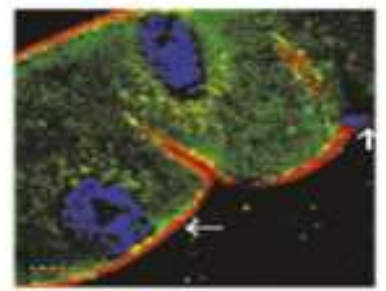

(f)

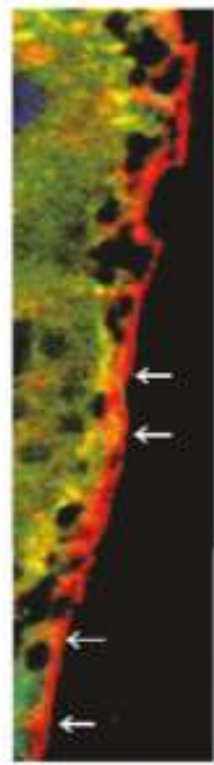

(k)

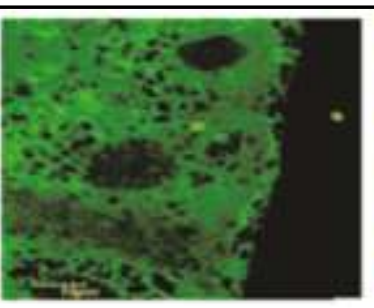

(c)

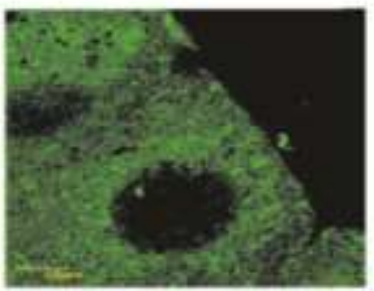

(g)

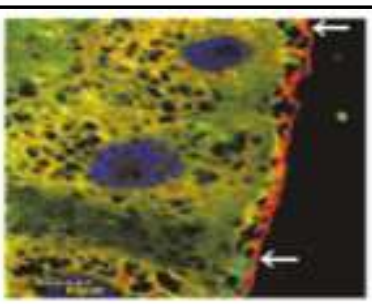

(d)

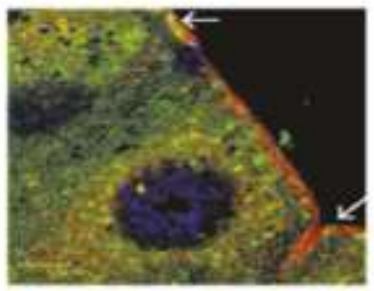

(h)

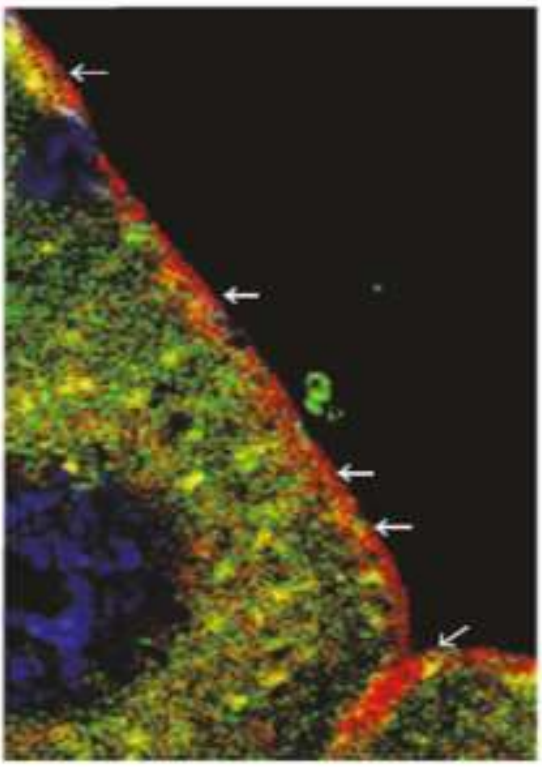

(1)

Figure 18. Cytoplasmic membrane-associated HNE-modified proteins in mutants. Color code: protein-HNE adducts, green; cytoplasmic membrane red; nuclei, blue. (a) Protein-HNE adducts in wild-type flies incubated at $20^{\circ} \mathrm{C}$. (b) Overlay with membrane staining. (c) ProteinHNE adducts in wild-type flies incubated at $29^{\circ} \mathrm{C}$. (d) Merged with membrane staining. (e), (f), (g), (h) Representative mutant, incubated at 20 or $29^{\circ} \mathrm{C}$ presented in the same order as in the upper row. White arrows in $(b),(d),(f)$, and $(h)$ point to regions displayed in higher magnification in $(i),(j),(k)$, and $(l)$, respectively. White arrows in $(i),(j),(k)$, and $(l)$ show association of the cytoplasmic membrane with alkylated proteins. Note the notorious infiltration of HNE-modified proteins into the membrane in (l), which occurs at a less extent in 
(k). The membrane of wild-type animals is free of alkylated proteins (i), while they associate closely with the membrane in the mutant $(j)$, incubated at the permissive temperature.

Drosophila models provide useful tools for exploring functional changes, pathomechanistic details, and genotype-phenotype correlations of some monogenic human disorders ${ }^{80}$ including mutations associated with inherited nephrotic syndromes and some of the known human genes involved in renal disease from flies to humans ${ }^{81}$. There are nephrotic manifestations of human COL4A1 mutations of the Hereditary Angiopathy, Nephropathy, Aneurysms, and Muscle Cramps (HANAC) syndrome ${ }^{38}$ and recent research revealed glomerular hyperpermeability and adult onset glomerulocystic kidney disease in association with COL4A1 mutations ${ }^{77}$. Some mechanistic elements, such as oxidative stress, have already been demonstrated in connection with type IV collagen mutations ${ }^{82}$. However, the evidence is quite limited for chronic inflammation and posttranslational protein modification and has so far only been detected in Drosophila col4al mutants ${ }^{67,62}$.

Mitochondrial fusion occurs when the cells are in a state of stress. Damaged mitochondria can fuse. This should be interpreted as an additional mechanism that rescues damaged organs and functions ${ }^{83}$. Our previous results have shown that the signs of cellular stress appear in the form of actin stress fibrils in Malpighian epithelial cells of the Drosophila col4al mutants ${ }^{62}$. The results of our current study show that mutation-associated stress is the key moment, and that triggers mitochondrial hyperfusion under restrictive conditions. Enlarged organelles are unevenly distributed within the cell, leading to a complete absence of mitochondria in some areas and a significant increase in others. A further consequence of the col4al mutation is that nitrated and alkylated proteins accumulate in the mutants and are localized to both normal and fused mitochondria.

These observations show that peroxynitrite-mediated nitrosative stress in col4al mutants produces higher concentrations of peroxynitrite ${ }^{67}$. Therefore, we hypothesized that elevated levels of peroxynitrite are likely to cause excess tyrosine nitration in proteins. However, this reaction does not consume peroxynitrite in col4al mutants. Furthermore, residual peroxynitrite in the system may initiate membrane damage by lipid peroxidation producing HNE, which in turn alkylates proteins by the mechanism of Michael addiction ${ }^{84}$. This theory is supported by the association of alkylated proteins with the epithelial cell 
membrane of Malpighian tubules. In addition, the TUNEL-positivity of nuclei confirms that mutation-induced stress leads the epithelia to degradation.

The results presented here clearly show that peroxynitrite plays a central role in col4alassociated defects. In wild-type animals and under physiological conditions, the nitrosoperoxycarbonate pathway is the preferential reaction of peroxynitrite, as the main decay products of nitrosoperoxycarbonate, nitrate anion, and carbon dioxide do not exert protein or membrane modification effects ${ }^{85}$. However, in mutants, elevated levels of peroxynitrite are above physiological concentrations and predominantly nitrate protein tyrosines, which, by forming HNE, leads to membrane damage, lipid peroxidation, protein alkylation, epithelial cell death, aberrant mitochondria, and Malpighian tubule dysfunction. 


\section{Conclusions}

As a result of mutagenesis by EMS, in our mutant series, the glycine was replaced with other amino acids in the COL4A1 protein. Two isoallelic variants col4a1 ${ }^{G 552 D 1}$, col4a1 ${ }^{G 552 D 2}$, and col4a1 $1^{G 1025 E 1}$, col4a1 ${ }^{G 1025 E 2}$ were identified. The consequence of the Gly552 residue is considered by the fact that a recent EMS mutagenesis resulted in the isolation of the same, temperature-sensitive, dominant-negative col4al ${ }^{G 552 D}$ allele ${ }^{86}$. Genotype-phenotype relationships identified in more than one hundred COLAAl mutants recognized in patients and murine models have shown that the position of the mutation and non-biochemical properties of the substitute amino acid have a greater influence on the phenotype and disease severity ${ }^{13}$. However, in our Drosophila mutant series, similar phenotypic errors were observed within the collagenous domain of the col4al gene, regardless of the site of mutation.

Because the phenotype of the common oviduct and the larval body wall muscle ${ }^{40}$, threatened the organization and deposition of actin, loss of sarcomere structure was observed in all the alleles tested, with features characteristic of myopathic or dystrophic states, with disintegrating muscle sarcomeres together with disintegration and streaming of Z-discs ${ }^{87}$. These morphological changes affect the function of the common oviduct, as females become sterilized and do not lay eggs ${ }^{40}$. Conditional knockout of genes in Drosophila, genes involved in integrin-mediated adhesion, including talin, alpha-actinin, integrin-linked kinase, alpha$P S 2$, and beta-PS integrins, Z-disc is leading to a common phenotype of streaming ${ }^{88}$, similar to our col4al mutant allele lines, indicating functional interdependence. Similarly, WalkerWarburg syndrome is diagnosed as a monogenic feature in patients with multiple gene mutations 49,89. Importantly, in Drosophila mutants deficient in protein O-mannosyl transferases, symptoms of the Walker-Warburg syndrome have been identified; streaming of Z-discs, actin filament disorganization and bundle formation have also been reported as part of the mutant phenotypes ${ }^{89}$.

Human myopathic/dystrophic states characterized by Z-disc streaming and sarcomeric disorganization include genes that, for genetic reasons, explicitly encode components of integrin-mediated adhesion. $I l k^{-/}$mouse embryos die during the peri-implantation phase due to polarization of the impaired epiblast polarisation and accumulation of F-actin at the integrin 
attachment sites ${ }^{90}$. Knockout mutants of the integrin beta subunits and most of the alpha subunits were generated with phenotypes ranging from the complete block in preimplantation, from developmental defects to perinatal lethality, demonstrating the specificity of each integrin. Muscular dystrophy has been observed in patients with ITGA5 or ITGA7 mutation ${ }^{91}$. However, Z-disc streaming has not been reported in association with ITGA5 or ITGA7 mutations. Mouse mutants of talin 1 or talin 2 undergo myopathy and sarcomere disassembly ${ }^{92}$. Nevertheless, the lethal embryonic mutation of the Drosophila rhea gene encoding talin recapitulates the phenotype of integrin beta PS mutations, illustrating their functional similarities ${ }^{93}$. These results indicate that genes involved in integrin-mediated adhesion are crucial, and homozygous recessive or null mutations are often lethal. The conditional lethality of the temperaturesensitive, heterozygous col4al Drosophila mutant series has allowed for the expression of phenotypic elements not detectable in humans or mice, such as confused sarcomeric cytoarchitecture and Z-disc streaming, which support the role of COL4A1 in integrin-mediated adhesion. In conclusion, our Drosophila mutant series can serve as a useful model for exploring how the COLAAI mutations disrupt myofiber-basement membrane interactions and compromise muscle function and provide biomarkers in therapeutic approaches.

The ancient form of the extracellular matrix may be the basement membrane, in which type IV collagen molecules played a key role in the transition from uni- to multicellularity ${ }^{11}$. Type IV collagen network provides BM visco-elasticity. Therefore, any malfunction of type IV collagen will compromise network elasticity and function. Throughout evolution, type IV collagens have been highly conserved, both structurally and functionally, including the mammalian - Drosophila relationship ${ }^{11}$. The ubiquitous mammalian and human BM contains (COL4A1) $)_{2} \mathrm{COL} 4 \mathrm{~A} 2$ heterotrimers and is distributed throughout the body. Glycine substitutions are triggers for systemic disease ${ }^{94}$. Genotype-phenotype relationships were associated with 93 COLAAI and 12 COLAA2 mutations. All lesions were dominant and represented approximately equal proportions of males (48\%) and females (52\%). Hereditary cases occur in $47 \%$ and sporadic cases in 53\%. As observed in other types of collagens, triplehelical Gly substitutions were the most common classes (68 out of 93 in COL4A1), frequent substitutions being charged amino acids; Arg (30), Glu (11) and Asp (10) ${ }^{13}$. The expression of phenotypic elements associated with the COLAAl mutation often depends on the genetic conditions of humans, mice, and flies. Key features of HANAC syndrome have been 
demonstrated in French families, with various heterozygous missense mutations in the COLAAI gene ${ }^{36}$. Retinal arteries tortuosity and retinal hemorrhage have been identified in a Spanish family, but the lesion was not associated with muscle spasms, renal or cerebral disorders, although the p.G510R mutation has previously been detected in a French family with HANAC syndrome ${ }^{95}$. COL4A1 splice site mutant C57BL6 / J mice were crossed with 129 / SvEvTac, and CAST / EiJ inbred strains and F1 generation offspring showed that they are phenotypically almost indistinguishable from the wild-type ${ }^{96}$. Similarly, the survival rates for the isoallelic mutants p.G552D1 and p.G552D2 differed slightly after heat selection. The p.G552D1 line survival rate was $26.9 \%$ for pupae and $5.3 \%$ for adults, whereas the p.G552D2 line survival rates were $5.7 \%$ and $0.50 \%{ }^{40,66}$.

The systemic phenotype of col4al mutations was provided, and evidence of Z-discs shifting, mitochondrial fusion, and loss of sarcomeres in the intestinal visceral muscle fibers was obtained. Genetic, genomic, and proteomic data suggest that the fruit fly contains all the genetic components of BM. Although all of the genetic elements of BM are less than mammals, thereby increasing the genetic utility of fly mutants for defective BM proteins, which have been conserved structurally and functionally throughout evolution, including type IV collagens ${ }^{6}$. The dominant col4al mutations developed BM-specific phenotypes that are more severe than those described in human and mouse models. The temperature sensitivity of the dominant col4a1 mutations are lethal condition that cannot be maintained in mammals. Furthermore, the phenotypic elements associated with col4al provide a number of biomarkers that can be quantitatively measured by screening a large number of drugs in a high-troughput Drosophila model aimed at alleviating the symptoms of type IV collagenopathy.

The Western blot was performed because our hypothesis was that the expression of COL4A1 protein was lower in the mutant flies. In my opinion, the gene carrying the point mutation adversely affects expression at the permissive temperature. However, no difference was found between adult wild-type and mutant animals at $20{ }^{\circ} \mathrm{C}$. In mutant adult animals, however, the amount of COL4A1 protein was apparently reduced at the restrictive temperature $\left(29^{\circ} \mathrm{C}\right)$. Earlier results from our group indicate that there was no decrease in the transcriptional level of col4a1 mRNA. These data suggest that decreasing levels of COL4A1 protein in the mutants at $29^{\circ} \mathrm{C}$ are caused by inadequate assembly and deposition. 
Previous Western blot experiments showed a decrease in COL4A1 protein in Drosophila col4al mutants at the restrictive temperature. We wanted to validate and visualize these results by immunofluorescence and were also interested in whether other proteins involved in the formation of the cytoskeletal-extracellular axis with COL4A1 exhibit a similar phenotype. The selected proteins were COL4A1, alpha subunits of integrin PS I and PS II, and Laminin gamma 1. We found that integrin staining and reduction in the midgut of mutant L3 larvae were at the permissive temperature. This phenomenon is exacerbated at restrictive temperatures, its distribution is irregular, and can be observed in adult animals. Laminin expression was also reduced at restrictive temperature in the midgut of mutant L3 larvae. The COL4A1 protein accumulates in the midgut of mutant animals and shows a further reduction, diffuse, and uneven staining at restrictive temperatures.

Following the common oviduct and the midgut, another organ was included in the study, the Malpighian tubule, which is analogous to the mammalian kidney. According to our extended hypothesis, the phenotype seen in the organs examined so far is also obtained in the Malpighian tubules, thus confirming the defective systemic character. Immunofluorescent staining was performed on the organs of adult animals at different ages ( 3 and 18 days old) and at different temperatures. The investigated proteins were the actin, COL4A1, and integrin alpha PS I and PSII subunits. Our recordings clearly showed that col4a1 mutants increase the number of actin stress fibrils as a result of restrictive temperature, decrease the amount of COL4A1 protein level, and the integrin distribution is uneven. Taken together, these results indicate that the Drosophila mutant col4al gene has an unfavorable phenotype at restrictive temperature, a systemic, multi-organ phenomenon.

As part of the stress response, Drosophila col4al mutants synthesize peroxynitrite above physiological concentrations. Excessive production of peroxynitrite initiates severe protein tyrosine nitration and protein alkylation, which adversely affects protein function, and also induces membrane lipid peroxidation and mitochondrial fusion. Wild-type control animals did not show these posttranslational modifications under physiological conditions because the nitrosoperoxycarbonate pathway was used to eliminate peroxynitrite. We suggest that in the mutant col4al Drosophila model, posttranslational protein modifications are an integral part of the col4a1-associated pathology and represent post mechanical details that have not yet been detected in humans or mice COL4A1 mutants. 


\section{$\underline{\text { Acknowledgement }}$}

First of all, I would like to thank my supervisors Dr Mátyás Mink and Prof. Dr Zsolt Boldogköi for their support and guidance throughout the PhD program.

I would like to thank my opponents, Professor Dr. Ferenc Deák and Dr. Gergő Groma, for their advice in helping me to write my thesis.

I would like to thank my help and support and time spent in our group with my current and former Ph.D. colleagues, Ildikó Valkonyné-Kelemen, Márton Kiss, and Nikoletta Somlyai-Popovics.

I would like to thank my colleagues and staff of the Department of Medical Biology for their assistance, especially for Dr Dóra Tombácz, Anikó Jász, Dr István Belecz, István Prazsák, Tamás Petkovics, Marianna Ábrahám, Csilla Magyarné-Papdi.

I am grateful for the effective teamwork for my Ph.D. student colleagues Dr Zsolt Csabai, Dr Zsolt Balázs, Norbert Moldován and Gábor Torma.

Last but not least, I would like to thank my aunt Katalin Dalmadi and my cousin Norbert Bugyi for their help. Without them, it would not have been possible. 


\section{$\underline{\text { References }}$}

1. Abedin M, King N. Diverse evolutionary paths to cell adhesion. Trends Cell Biol. 2010. doi:10.1016/j.tcb.2010.08.002

2. Schmidt $S$, Friedl P. Interstitial cell migration: Integrin-dependent and alternative adhesion mechanisms. Cell Tissue Res. 2010. doi:10.1007/s00441-009-0892-9

3. Frantz C, Stewart KM, Weaver VM. The extracellular matrix at a glance. J Cell Sci. 2010. doi:10.1242/jcs.023820

4. Rozario T, DeSimone DW. The extracellular matrix in development and morphogenesis: A dynamic view. Dev Biol. 2010. doi:10.1016/j.ydbio.2009.10.026

5. Pozzi A, Yurchenco PD, Iozzo R V. The nature and biology of basement membranes. Matrix Biol. 2017;57-58:1-11. doi:10.1016/j.matbio.2016.12.009

6. Ramos-Lewis W, Page-McCaw A. Basement membrane mechanics shape development: Lessons from the fly. Matrix Biol. April 2018. doi:10.1016/j.matbio.2018.04.004

7. Humphries MJ, Travis MA, Clark K, Mould AP. Mechanisms of integration of cells and extracellular matrices by integrins. In: Biochemical Society Transactions. ; 2004. doi:10.1042/BST0320822

8. Paulson M. Basement membrane proteins: Structure, assembly, and cellular interactions. Crit Rev Biochem Mol Biol. 1992. doi:10.3109/10409239209082560

9. Khoshnoodi J, Pedchenko V, Hudson BG. Mammalian collagen IV. Microsc Res Tech. 2008. doi:10.1002/jemt.20564

10. Ricard-Blum S. The Collagen Family. Cold Spring Harb Perspect Biol. 2011. doi:10.1101/cshperspect.a004978

11. Fidler AL, Darris CE, Chetyrkin S V., et al. Collagen iv and basement membrane at the evolutionary dawn of metazoan tissues. Elife. 2017. doi:10.7554/eLife.24176.001

12. Heikkilä P, Soininen R. The type IV collagen gene family. Contrib Nephrol. 1996;117(Iv):105-129. 
13. Jeanne M, Gould DB. Genotype-phenotype correlations in pathology caused by collagen type IV alpha 1 and 2 mutations. Matrix Biol. 2017;57-58:29-44. doi:10.1016/j.matbio.2016.10.003

14. Ricard-Blum S, Ruggiero F. The collagen superfamily: From the extracellular matrix to the cell membrane. Pathol Biol. 2005. doi:10.1016/j.patbio.2004.12.024

15. Myllyharju J, Kivirikko KI, Kivirikko KI, et al. Collagens, modifying enzymes and their mutations in humans, flies and worms. Trends Genet. 2004. doi:10.1016/j.tig.2003.11.004

16. Fidler AL, Boudko SP, Rokas A, Hudson BG. The triple helix of collagens - An ancient protein structure that enabled animal multicellularity and tissue evolution. $J$ Cell Sci. 2018. doi:10.1242/jcs.203950

17. Kiss AA, Somlyai-Popovics N, Kiss M, Boldogkői Z, Csiszár K, Mink M. Type IV Collagen Is Essential for Proper Function of Integrin-Mediated Adhesion in Drosophila Muscle Fibers. Int J Mol Sci. 2019;20(20). doi:10.3390/ijms20205124

18. Morrissey MA, Sherwood DR. An active role for basement membrane assembly and modification in tissue sculpting. J Cell Sci. 2015. doi:10.1242/jcs.168021

19. Boutaud A, Borza DB, Bondar O, et al. Type IV collagen of the glomerular basement membrane. Evidence that the chain specificity of network assembly is encoded by the noncollagenous NC1 domains. J Biol Chem. 2000. doi:10.1074/jbc.M004569200

20. Borza DB, Bondar O, Ninomiya Y, et al. The NC1 Domain of Collagen IV Encodes a Novel Network Composed of the $\alpha 1, \alpha 2, \alpha 5$, and $\alpha 6$ Chains in Smooth Muscle Basement Membranes. J Biol Chem. 2001. doi:10.1074/jbc.M103690200

21. Hudson BG, Tryggvason K, Sundaramoorthy M, Neilson EG. Alport's Syndrome, Goodpasture's Syndrome, and Type IV Collagen. N Engl J Med. 2003;348(25):25432556. doi:10.1056/NEJMra022296

22. Gillies AR, Lieber RL. Structure and function of the skeletal muscle extracellular matrix. Muscle and Nerve. 2011. doi:10.1002/mus.22094

23. Guiraud S, Migeon T, Ferry A, et al. HANAC Col4a1 Mutation in Mice Leads to 
Skeletal Muscle Alterations due to a Primary Vascular Defect. Am J Pathol. 2017;187(3):505-516. doi:10.1016/j.ajpath.2016.10.020

24. Birnkrant DJ, Bushby K, Bann CM, et al. Diagnosis and management of Duchenne muscular dystrophy, part 1: diagnosis, and neuromuscular, rehabilitation, endocrine, and gastrointestinal and nutritional management. Lancet Neurol. 2018. doi:10.1016/S1474-4422(18)30024-3

25. Birnkrant DJ, Bushby K, Bann CM, et al. Diagnosis and management of Duchenne muscular dystrophy, part 2: respiratory, cardiac, bone health, and orthopaedic management. Lancet Neurol. 2018. doi:10.1016/S1474-4422(18)30025-5

26. Bertini E, D’Amico A, Gualandi F, Petrini S. Congenital Muscular Dystrophies: A Brief Review. Semin Pediatr Neurol. 2011. doi:10.1016/j.spen.2011.10.010

27. Alport AC. HEREDITARY FAMILIAL CONGENITAL HAEMORRHAGIC NEPHRITIS. Br Med J. 1927;1(3454):504-506.

http://www.ncbi.nlm.nih.gov/pubmed/20773074. Accessed July 5, 2018.

28. Tryggvason K, Zhou J, Hostikka SL, Shows TB. Molecular genetics of Alport syndrome. Kidney Int. 1993;43(1):38-44.

http://www.ncbi.nlm.nih.gov/pubmed/8433568. Accessed July 5, 2018.

29. Nozu K, Nakanishi K, Abe Y, et al. A review of clinical characteristics and genetic backgrounds in Alport syndrome. Clin Exp Nephrol. 2019. doi:10.1007/s10157-018$1629-4$

30. Lee J, Nozu K, Choi D, Kang H, Ha I-S, Cheong H. Features of Autosomal Recessive Alport Syndrome: A Systematic Review. J Clin Med. 2019. doi:10.3390/jcm8020178

31. Funk SD, Lin MH, Miner JH. Alport syndrome and Pierson syndrome: Diseases of the glomerular basement membrane. Matrix Biol. 2018. doi:10.1016/j.matbio.2018.04.008

32. McAdoo SP, Pusey CD. Anti-glomerular basement membrane disease. Clin J Am Soc Nephrol. 2017. doi:10.2215/CJN.01380217

33. Greco A, Rizzo MI, De Virgilio A, et al. Goodpasture's syndrome: A clinical update. Autoimmun Rev. 2015. doi:10.1016/j.autrev.2014.11.006 
34. Pedchenko V, Kitching AR, Hudson BG. Goodpasture's autoimmune disease - A collagen IV disorder. Matrix Biol. 2018. doi:10.1016/j.matbio.2018.05.004

35. Vahedi K, Alamowitch S. Clinical spectrum of type IV collagen (COL4A1) mutations: A novel genetic multisystem disease. Curr Opin Neurol. 2011.

doi:10.1097/WCO.0b013e32834232c6

36. Plaisier E, Chen Z, Gekeler F, et al. Novel COL4A1 mutations associated with HANAC syndrome: a role for the triple helical CB3[IV] domain. Am J Med Genet A. 2010;152A(10):2550-2555. doi:10.1002/ajmg.a.33659

37. Plaisier E, Ronco P. COL4A1-Related Disorders.; 1993.

38. Plaisier E, Gribouval O, Alamowitch S, et al. COLAA1 Mutations and Hereditary Angiopathy, Nephropathy, Aneurysms, and Muscle Cramps. N Engl J Med. 2007;357(26):2687-2695. doi:10.1056/NEJMoa071906

39. Kelemen-Valkony I, Kiss M, Csiha J, et al. Drosophila basement membrane collagen col4a1 mutations cause severe myopathy. Matrix Biol. 2012;31(1):29-37. doi:10.1016/j.matbio.2011.09.004

40. Kelemen-Valkony I, Kiss M, Csiha J, et al. Drosophila basement membrane collagen col4a1 mutations cause severe myopathy. Matrix Biol. 2012;31(1):29-37. doi:10.1016/J.MATBIO.2011.09.004

41. Lajeunesse DR, Buckner SM, Lake J, Na C, Pirt A, Fromson K. Three new Drosophila markers of intracellular membranes. Biotechniques. 2004;36(5):784-790. doi:10.2144/04365ST01

42. Mishra M, Tiwari S, Gomes A V. Protein purification and analysis: Next generation western blotting techniques. Expert Rev Proteomics. 2017. doi:10.1080/14789450.2017.1388167

43. Ghosh R, Gilda JE, Gomes A V. The necessity of and strategies for improving confidence in the accuracy of western blots. Expert Rev Proteomics. 2014. doi:10.1586/14789450.2014.939635

44. Bradford MM. A rapid and sensitive method for the quantitation of microgram 
quantities of protein utilizing the principle of protein-dye binding. Anal Biochem. 1976;72:248-254. http://www.ncbi.nlm.nih.gov/pubmed/942051. Accessed July 5, 2018.

45. Fessler LI, Condic ML, Nelson RE, Fessler JH, Fristrom JW. Site-specific cleavage of basement membrane collagen IV during Drosophila metamorphosis. Development. 1993;117(3):1061-1069. http://www.ncbi.nlm.nih.gov/pubmed/8325235. Accessed July 5, 2018.

46. Pöschl E, Schlötzer-Schrehardt U, Brachvogel B, et al. Collagen IV is essential for basement membrane stability but dispensable for initiation of its assembly during early development. Development. 2004;131(7):1619-1628. doi:10.1242/dev.01037

47. Gupta MC, Graham PL, Kramer JM. Characterization of $\alpha$ (IV) collagen mutations in Caenorhabditis elegans and the effects of $\alpha 1$ and $\alpha 2(\mathrm{IV})$ mutations on type IV collagen distribution. J Cell Biol. 1997. doi:10.1083/jcb.137.5.1185

48. Kashtan CE. Alport syndromes: phenotypic heterogeneity of progressive hereditary nephritis. Pediatr Nephrol. 2000;14(6):0502-0512. doi:10.1007/s004670050804

49. Labelle-Dumais C, Dilworth DJ, Harrington EP, et al. COL4A1 mutations cause ocular dysgenesis, neuronal localization defects, and myopathy in mice and Walker-Warburg syndrome in humans. Spinner NB, ed. PLoS Genet. 2011;7(5):e1002062. doi:10.1371/journal.pgen.1002062

50. Jeannet P-Y, Bassez G, Eymard B, et al. Clinical and histologic findings in autosomal centronuclear myopathy. Neurology. 2004;62(9):1484-1490. http://www.ncbi.nlm.nih.gov/pubmed/15136669. Accessed July 5, 2018.

51. Dowling JJ, Vreede AP, Low SE, et al. Loss of Myotubularin Function Results in TTubule Disorganization in Zebrafish and Human Myotubular Myopathy. Cox GA, ed. PLoS Genet. 2009;5(2):e1000372. doi:10.1371/journal.pgen.1000372

52. Tosch V, Rohde HM, Tronchère H, et al. A novel PtdIns3P and PtdIns(3,5)P2 phosphatase with an inactivating variant in centronuclear myopathy. Hum Mol Genet. 2006;15(21):3098-3106. doi:10.1093/hmg/ddl250

53. Nicot A-S, Toussaint A, Tosch V, et al. Mutations in amphiphysin 2 (BIN1) disrupt 
interaction with dynamin 2 and cause autosomal recessive centronuclear myopathy. Nat Genet. 2007;39(9):1134-1139. doi:10.1038/ng2086

54. Bitoun M, Maugenre S, Jeannet P-Y, et al. Mutations in dynamin 2 cause dominant centronuclear myopathy. Nat Genet. 2005;37(11):1207-1209. doi:10.1038/ng1657

55. Leventis PA, Chow BM, Stewart BA, Iyengar B, Campos AR, Boulianne GL. Drosophila Amphiphysin is a post-synaptic protein required for normal locomotion but not endocytosis. Traffic. 2001;2(11):839-850.

http://www.ncbi.nlm.nih.gov/pubmed/11733051. Accessed July 5, 2018.

56. Razzaq A, Robinson IM, McMahon HT, et al. Amphiphysin is necessary for organization of the excitation-contraction coupling machinery of muscles, but not for synaptic vesicle endocytosis in Drosophila. Genes Dev. 2001;15(22):2967-2979. doi:10.1101/gad.207801

57. Masur SK, Kim YT, Wu CF. Reversible inhibition of endocytosis in cultured neurons from the Drosophila temperature-sensitive mutant shibirets1. J Neurogenet. 1990;6(3):191-206. http://www.ncbi.nlm.nih.gov/pubmed/2113575. Accessed July 5, 2018.

58. Gould DB, Phalan FC, Breedveld GJ, et al. Mutations in Col4a1 Cause Perinatal Cerebral Hemorrhage and Porencephaly. Science (80- ). 2005;308(5725):1167-1171. doi:10.1126/science. 1109418

59. Gould DB, Phalan FC, van Mil SE, et al. Role of COL4A1 in Small-Vessel Disease and Hemorrhagic Stroke. N Engl J Med. 2006;354(14):1489-1496. doi:10.1056/NEJMoa053727

60. Breedveld G, de Coo IF, Lequin MH, et al. Novel mutations in three families confirm a major role of COL4A1 in hereditary porencephaly. J Med Genet. 2006;43(6):490-495. doi:10.1136/jmg.2005.035584

61. Rui Y, Bai J, Perrimon N. Sarcomere formation occurs by the assembly of multiple latent protein complexes. PLoS Genet. 2010. doi:10.1371/journal.pgen.1001208

62. Kiss AA, Popovics N, Szabó G, Csiszár K, Mink M. Altered stress fibers and integrin expression in the Malpighian epithelium of Drosophila type IV collagen mutants. Data 
Br. 2016;7. doi:10.1016/j.dib.2016.03.059

63. Kiss AA, Somlyai-Popovics N, Tubak V, Boldogkoi Z, Csiszár K, Mink M. Novel phenotypic elements of type IV collagenopathy revealed by the Drosophila model. Appl Sci. 2019. doi:10.3390/app9102083

64. Suzuki DT, Procunier D. Temperature-sensitive mutations in Drosophila melanogaster. 3. Dominant lethals and semilethals on chromosome 2. Proc Natl Acad Sci U S A. 1969;62(2):369-376. http://www.ncbi.nlm.nih.gov/pubmed/5256216. Accessed July 5, 2018.

65. Kelemen-Valkony I, Kiss M, Csiszár K, Mink M. Inherited myopathies. In: Myopathies: New Research. ; 2013.

66. Kiss AA, Popovics N, Marton K, Boldogkoi Z, Csiszar K, Mink M. Type IV collagen is essential for proper function of integrin-mediated adhesion in Drosophila muscle fibers. bioRxiv. May 2018:318337. doi:10.1101/318337

67. Kiss M, Kiss AA, Radics M, et al. Drosophila type IV collagen mutation associates with immune system activation and intestinal dysfunction. Matrix Biol. 2016;49:120131. doi:10.1016/j.matbio.2015.09.002

68. Kiss AA, Popovics N, Boldogki Z, Csiszár K, Mink M. 4-Hydroxy-2-nonenal Alkylated and Peroxynitrite Nitrated Proteins Localize to the Fused Mitochondria in Malpighian Epithelial Cells of Type IV Collagen Drosophila Mutants. Biomed Res Int. 2018;2018. doi:10.1155/2018/3502401

69. Washington HS, Jimenez CEC. Myopathies : New Research. Nova Science Publishers; 2012. https://www.novapublishers.com/catalog/product_info.php?products_id=35505. Accessed July 5, 2018.

70. Sorokin L. The impact of the extracellular matrix on inflammation. Nat Rev Immunol. 2010;10(10):712-723. doi:10.1038/nri2852

71. Altincicek B, Vilcinskas A. Metamorphosis and collagen-IV-fragments stimulate innate immune response in the greater wax moth, Galleria mellonella. Dev Comp Immunol. 2006;30(12):1108-1118. doi:10.1016/J.DCI.2006.03.002 
72. Altincicek B, Berisha A, Mukherjee K, Spengler B, Römpp A, Vilcinskas A. Identification of collagen IV derived danger/alarm signals in insect immunity by nanoLC-FTICR MS. Biol Chem. 2009;390(12):1303-1311. doi:10.1515/BC.2009.128

73. Hynes RO, Zhao Q. The evolution of cell adhesion. J Cell Biol. 2000;150(2):F89-96. doi:10.1083/JCB.150.2.F89

74. Molnar J, Ujfaludi Z, T Fong SF, et al. Drosophila Lysyl Oxidases Dmloxl-1 and Dmloxl-2 Are Differentially Expressed and the Active DmLOXL-1 Influences Gene Expression and Development*. 2005. doi:10.1074/jbc.M503006200

75. Molnar J, Fong KSK, He QP, et al. Structural and functional diversity of lysyl oxidase and the LOX-like proteins. Biochim Biophys Acta - Proteins Proteomics. 2003;1647(12):220-224. doi:10.1016/S1570-9639(03)00053-0

76. Dow JAT, Romero MF. Drosophila provides rapid modeling of renal development, function, and disease. Am J Physiol Physiol. 2010;299(6):F1237-F1244. doi:10.1152/ajprenal.00521.2010

77. Chen Z, Migeon T, Verpont M-C, et al. HANAC Syndrome Col4a1 Mutation Causes Neonate Glomerular Hyperpermeability and Adult Glomerulocystic Kidney Disease. $J$ Am Soc Nephrol. 2016;27(4):1042-1054. doi:10.1681/ASN.2014121217

78. Wickström SA, Radovanac K, Fässler R. Genetic analyses of integrin signaling. Cold Spring Harb Perspect Biol. 2011;3(2):a005116. doi:10.1101/cshperspect.a005116

79. Radi R. Nitric oxide, oxidants, and protein tyrosine nitration. Proc Natl Acad Sci U S A. 2004;101(12):4003-4008. doi:10.1073/pnas.0307446101

80. Chien S, Reiter LT, Bier E, Gribskov M. Homophila: human disease gene cognates in Drosophila. Nucleic Acids Res. 2002;30(1):149-151. http://www.ncbi.nlm.nih.gov/pubmed/11752278. Accessed July 5, 2018.

81. Fu Y, Zhu J, Richman A, et al. A Drosophila model system to assess the function of human monogenic podocyte mutations that cause nephrotic syndrome. Hum Mol Genet. 2017;26(4):768-780. doi:10.1093/hmg/ddw428

82. Weng Y-C, Dilworth DJ, Libby RT, John SWM, Gould DB. Mutant COL4A1 triggers 
oxidative stress in a genetic model of AMD. Matrix Biol. 2008;27:39.

doi:10.1016/J.MATBIO.2008.09.339

83. Youle RJ, Van Der Bliek AM. Mitochondrial fission, fusion, and stress. Science (80- ). 2012. doi:10.1126/science. 1219855

84. Schopfer FJ, Baker PRS, Freeman BA. NO-dependent protein nitration: A cell signaling event or an oxidative inflammatory response? Trends Biochem Sci. 2003. doi:10.1016/j.tibs.2003.10.006

85. Nash KM, Rockenbauer A, Villamena FA. Reactive nitrogen species reactivities with nitrones: Theoretical and experimental studies. Chem Res Toxicol. 2012. doi:10.1021/tx200526y

86. Hollfelder D, Frasch M, Reim I. Distinct functions of the laminin $\beta$ LN domain and collagen IV during cardiac extracellular matrix formation and stabilization of alary muscle attachments revealed by EMS mutagenesis in Drosophila. BMC Dev Biol. 2014. doi:10.1186/1471-213X-14-26

87. Rahimov F, Kunkel LM. Cellular and molecular mechanisms underlying muscular dystrophy. J Cell Biol. 2013. doi:10.1083/jcb.201212142

88. Perkins AD, Ellis SJ, Asghari P, Shamsian A, Moore EDW, Tanentzapf G. Integrinmediated adhesion maintains sarcomeric integrity. Dev Biol. 2010. doi:10.1016/j.ydbio.2009.10.034

89. Ueyama M, Akimoto Y, Ichimiya T, et al. Increased apoptosis of myoblasts in Drosophila model for the Walker-Warburg syndrome. PLoS One. 2010. doi:10.1371/journal.pone.0011557

90. Sakai T, Li S, Docheva D, et al. Integrin-linked kinase (ILK) is required for polarizing the epiblast, cell adhesion, and controlling actin accumulation. Genes Dev. 2003. doi:10.1101/gad.255603

91. Hynes RO. Integrins: Bidirectional, allosteric signaling machines. Cell. 2002. doi:10.1016/S0092-8674(02)00971-6

92. Conti FJ, Monkley SJ, Wood MR, Critchley DR, Müller U. Talin 1 and 2 are required 
for myoblast fusion, sarcomere assembly and the maintenance of myotendinous junctions. Development. 2009. doi:10.1242/dev.035857

93. Brown NH, Gregory SL, Rickoll WL, et al. Talin is essential for integrin function in Drosophila. Dev Cell. 2002. doi:10.1016/S1534-5807(02)00290-3

94. Yurchenco PD, Patton BL. Developmental and pathogenic mechanisms of basement membrane assembly. Curr Pharm Des. 2009;15(12):1277-1294. http://www.ncbi.nlm.nih.gov/pubmed/19355968. Accessed December 26, 2018.

95. Zenteno JC, Crespí J, Buentello-Volante B, et al. Next generation sequencing uncovers a missense mutation in COL4A1 as the cause of familial retinal arteriolar tortuosity. Graefe's Arch Clin Exp Ophthalmol. 2014;252(11):1789-1794. doi:10.1007/s00417014-2800-6

96. Gould DB, Marchant JK, Savinova O V, Smith RS, John SWM. Col4a1 mutation causes endoplasmic reticulum stress and genetically modifiable ocular dysgenesis. Hum Mol Genet. 2007;16(7):798-807. doi:10.1093/hmg/ddm024 


\section{Article I.}

Drosophila basement membrane collagen col4a1 mutations cause severe myopathy

Kelemen-Valkony I ${ }^{\mathrm{a}}$, Kiss $\mathrm{M}^{\mathrm{a}}$, Csiha $\mathrm{J}^{\mathrm{a}}$, Kiss A $\mathbf{A}^{\mathrm{a}}$, Bircher $\mathrm{U}^{\mathrm{a}}$, Szidonya $\mathrm{J}^{\mathrm{a}}$, Maróy Pa Juhász $\mathrm{G}^{\mathrm{c}}$, Komonyi $\mathrm{O}^{\mathrm{a}}$, Csiszár $\mathrm{K}^{\mathrm{b}}$, Mink $\mathrm{M}^{\mathrm{a},{ }^{*}}$

Matrix Biology (2012)

https://doi.org/10.1016/j.matbio.2011.09.004

MTMT azonosító: 2104514

IF: $3.19(Q 2)$ 


\title{
Drosophila basement membrane collagen col4a1 mutations cause severe myopathy
}

\author{
Ildikó Kelemen-Valkony a , Márton Kiss a , Judit Csiha a , András Kiss a , Urs Bircher a , János Szidonya a , \\ Péter Maróy ${ }^{\mathrm{a}}$, Gábor Juhász ${ }^{\mathrm{c}}$, Orbán Komonyi ${ }^{\mathrm{a}}$, Katalin Csiszár ${ }^{\mathrm{b}}$, Mátyás Mink ${ }^{\mathrm{a}, *}$ \\ a Department of Genetics, University of Szeged, Középfasor 52, H-6726 Szeged, Hungary \\ ${ }^{\mathrm{b}}$ John A. Burns School of Medicine, University of Hawaii, 1960 East West Road, Honolulu, HI 96822, USA \\ c Department of General Zoology, Eötvös Loránd University, Pázmány sétány 1/C, H-1117 Budapest, Hungary
}

\section{A R T I C L E I N F O}

\section{Article history:}

Received 7 March 2011

Received in revised form 20 September 2011

Accepted 26 September 2011

\section{Keywords:}

Type IV collagen

Drosophila

Myopathy

Multiple mutations

\begin{abstract}
A B S T R A C T
Recent data from clinical and mammalian genetic studies indicate that COL4A1 mutations manifest with basement membrane defects that result in muscle weakness, cramps, contractures, dystrophy and atrophy. Indepth studies of mutant COL4A1-associated muscle phenotype, however, are lacking and significant details of the muscle-specific pathomechanisms remain unknown. In this study, we have used a comprehensive set of Drosophila col4a1 and col4a2 mutants and a series of genetic and mutational analyses, gene, protein expression, and immunohistochemistry experiments in order to establish a Drosophila model and address some of these questions. The Drosophila genome contains two type IV collagen genes, col4a1 and col4a2. Mutant heterozygotes of either gene are viable and fertile, whereas homozygotes are lethal. In complementation analysis of all known mutants of the locus and a complementation matrix derived from these data we have identified the dominant lesions within the col4a1, but not within the col4a2 gene. Expression of a col4a1 transgene partially rescued the dominant and recessive mutant col4a1 alleles but not the col4a2 mutations that were all recessive. Partial complementation suggested that col4a1 gene mutations have strong antimorph effect likely due to the incorporation of the mutant protein into the triple helix. In col4a1 mutants, morphological changes of the oviduct muscle included severe myopathy with centronuclear myofibers leading to gradual development of female sterility. In larval body wall muscles ultrastructural changes included disturbance of $\mathrm{A}$ and I bands between persisting $\mathrm{Z}$ bands. In the most severely affected DTS-L3 mutant, we have identified four missense mutations within the coding region of the col4a1 gene two of which affected the Y within the Gly-X-Y unit and a 3' UTR point mutation. In conclusion, our Drosophila mutant series may serve as an effective model to uncover the mechanisms by which COL4A1 mutations result in compromised myofiber-basement membrane interactions and aberrant muscle function.
\end{abstract}

(c) 2011 Elsevier B.V. All rights reserved.

\section{Introduction}

Type IV collagen, along with various other components, is a ubiquitous component of the basement membrane (BM). The BM is involved in diverse physiological functions including protection, absorption, secretion, sensation, and separation of animal tissues and body compartments. Mammals harbor three pairs of type IV collagen genes, COL4A1 through COL4A6, with each gene pair in head-tohead arrangement (Sado et al., 1998; Kashtan, 1999; Miosge, 2001). Mutations in the X-linked COL4A5 gene account for about $80 \%$ of patients with Alport syndrome, the inherited disorder of renal, ocular and cochlear basement membranes (Alport, 1927; Tryggvason et al., 1993). Lesions of the autosomal COL4A3 and COL4A4 genes are responsible for the symptoms of Alport syndrome in the remaining $20 \%$ of patients. The available animal models for collagen type IV

\footnotetext{
* Corresponding author. Tel.: + 3662 544269; fax: + 3662544651 E-mail address: mink@bio.u-szeged.hu (M. Mink).
}

mutations present phenocopies of the human COL4-associated diseases. Introduction of known human mutations in the X-linked Col4a5 gene results in sexual dimorphism and symptoms of Alport syndrome in mice (Kashtan, 1999; Ortega and Werb, 2002; Rheault et al., 2004). Col4a1/2-/- mice, null for the homologous pair of the collagen $4 a 1$ and $4 a 2$ genes, demonstrate arrest in early embryonic development (Pöschl et al., 2004). Both mouse Col4a1 and Col4a2 and human COL4A1 and COL4A2 mutations (Favor et al., 2007) are phenotypically pleiotropic in their clinical manifestations. Missense or intron splicing alleles of human COL4A1 are linked to vascular instability, aneurysm formation and intracerebral hemorrhage (Gould et al., 2005; Van Agtmael et al., 2005; Gould et al., 2006; Vahedi et al., 2007) and affect several other tissues and organs, including kidney, eye and muscles (Plaisier et al., 2007).

In Drosophila, there are two type IV collagen genes, col4a1 and col4a2 within the $25 \mathrm{C}$ band of the second chromosome. Previous saturating ethyl-methane-sulfonate (EMS) mutagenesis revealed an allelic dominant temperature-sensitive (DTS) mutational hot-spot, the lethal $l(2) 25 \mathrm{Ca}$ locus, within the 25C band (Suzuki and Procunier, 
Table 1

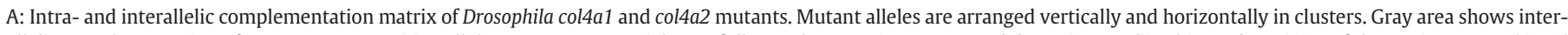

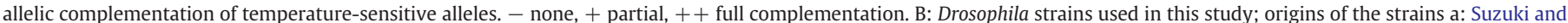

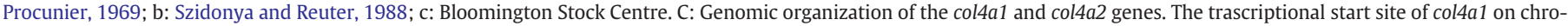
mosome 2, left arm (2L), is at nucleotide position 5029615, col4a2 transcription starts at 5027412. Both gene sequences refer to RefSeq NM_164615 and NM_057842 entries.

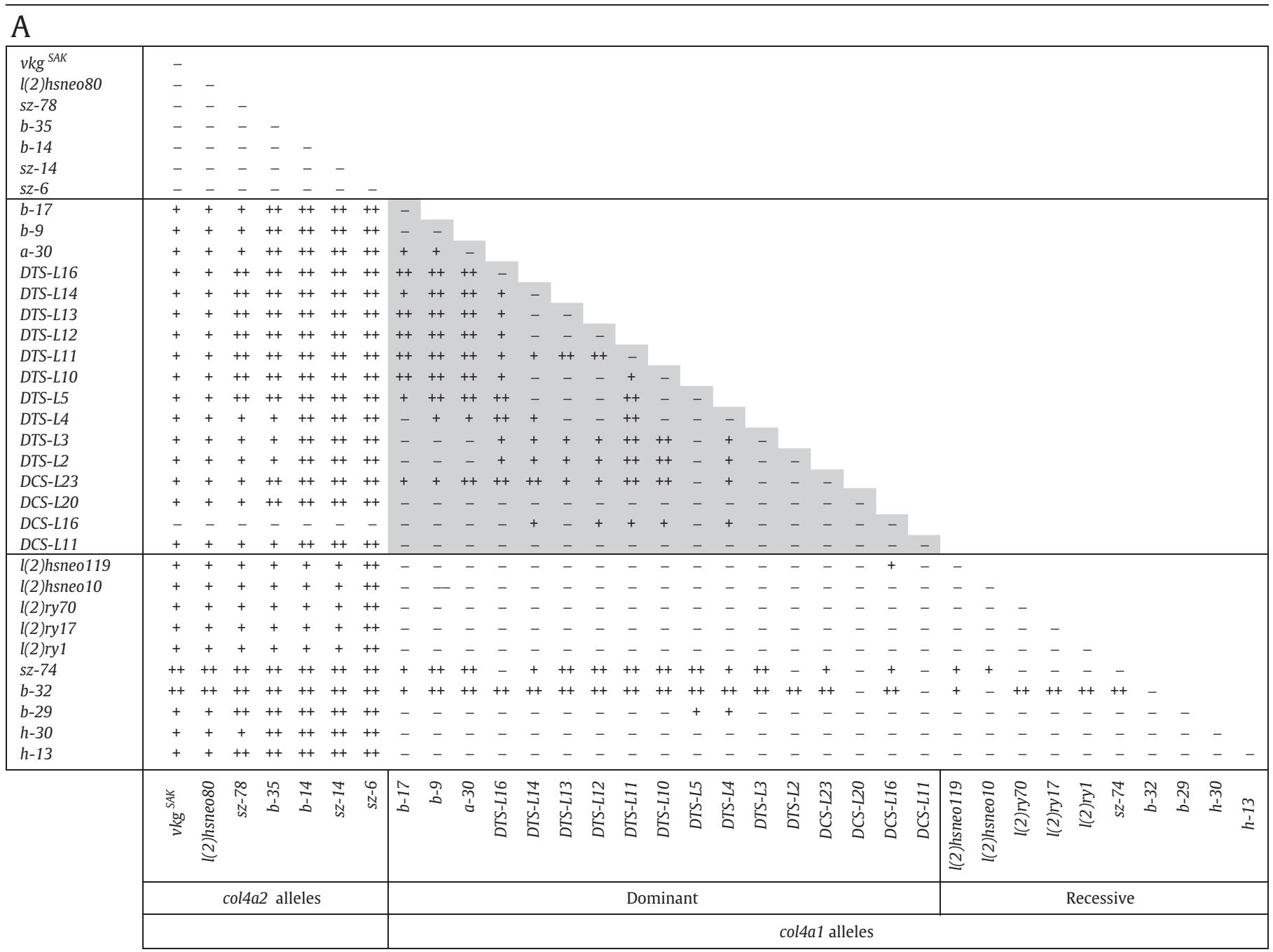

B

\begin{tabular}{lll}
\hline $\begin{array}{l}\text { Dominant } \\
\text { alleles }\end{array}$ & $\begin{array}{l}\text { Recessive } \\
\text { alleles }\end{array}$ & $\begin{array}{l}\text { P-insertion } \\
\text { alleles }\end{array}$ \\
\hline$D C S-L 11^{\mathrm{a}}$ & $h-13^{\mathrm{b}}$ & $l(2) h s n e 010^{\mathrm{c}}$ \\
$D C S-L 16^{\mathrm{a}}$ & $h-30^{\mathrm{b}}$ & $l(2) h s n e 0119^{\mathrm{c}}$ \\
$D C S-L 20^{\mathrm{a}}$ & $b-14^{\mathrm{b}}$ & $l(2) h s n e 080^{\mathrm{c}}$ \\
$D C S-L 23^{\mathrm{a}}$ & $b-29^{\mathrm{b}}$ & $v \mathrm{~S}^{\mathrm{b} A K c}$ \\
$D T S-L 2^{\mathrm{a}}$ & $b-32^{\mathrm{b}}$ & \\
$D T S-L 3^{\mathrm{a}}$ & $b-35^{\mathrm{b}}$ & P-revertants \\
$D T S-L 4^{\mathrm{a}}$ & $s z-6^{\mathrm{b}}$ & \\
$D T S-L 5^{\mathrm{a}}$ & $s z-14^{\mathrm{b}}$ & $l(2) r y-1^{\mathrm{b}}$ \\
$D T S-L 10^{\mathrm{a}}$ & $s z-74^{\mathrm{b}}$ & $l(2) r y-17^{\mathrm{b}}$ \\
$D T S-L 11^{\mathrm{a}}$ & $s z-78^{\mathrm{b}}$ & \\
$D T S-L 12^{\mathrm{a}}$ & & \\
$D T S-L 13^{\mathrm{a}}$ & & \\
$D T S-L 14^{\mathrm{a}}$ & & \\
$D T S-L 16^{\mathrm{a}}$ & & \\
$a-30^{\mathrm{b}}$ & & \\
$b-9^{\mathrm{b}}$ & & \\
$b-17^{\mathrm{b}}$ & & \\
\hline
\end{tabular}

C

25C1 band, $l(2) 25 C a$ locus

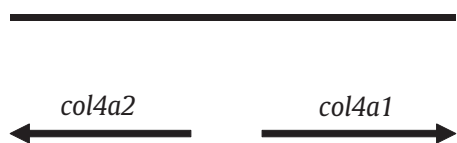

1969; Suzuki, 1970). Independent EMS mutagenesis data and identification of additional DTS alleles within the 25C band further supported the presence of a mutational hot-spot in this chromosomal location
(Szidonya and Reuter, 1988). Heterozygotes $l(2) 25 C a$ DTS/+ or $l(2)$ $25 \mathrm{Ca} D C S /+$ are sensitive to heat or cold and die at restrictive temperatures. The onset of the heat-sensitive period is in late embryos and 
Table 2

Percentage of offspring of dominant temperature-sensitive mutants at $29{ }^{\circ} \mathrm{C}$ relative to those incubated at $20^{\circ} \mathrm{C}$. Results represent averages of data from three independent experiments involving 900 embryos.

\begin{tabular}{|c|c|c|c|c|c|}
\hline $\begin{array}{l}\text { col4a1 } \\
\text { allele }\end{array}$ & $\begin{array}{l}\text { Pupae } \\
29^{\circ} \mathrm{C}\end{array}$ & $\begin{array}{l}\text { Adults } \\
29{ }^{\circ} \mathrm{C}\end{array}$ & $\begin{array}{l}\text { col4a1 } \\
\text { allele }\end{array}$ & $\begin{array}{l}\text { Pupae } \\
29{ }^{\circ} \mathrm{C}\end{array}$ & $\begin{array}{l}\text { Adults } \\
29^{\circ} \mathrm{C}\end{array}$ \\
\hline DTS-L3 & $5.7 \%$ & $0.5 \%$ & $a-30$ & $41.7 \%$ & $7.4 \%$ \\
\hline DTS-L4 & $19.5 \%$ & $1.8 \%$ & DTS-L14 & $37.8 \%$ & $10.0 \%$ \\
\hline DTS-L10 & $22.7 \%$ & $2.9 \%$ & DTS-L13 & $40.6 \%$ & $11.5 \%$ \\
\hline DTS-L5 & $32.9 \%$ & $3.3 \%$ & DTS-L16 & $54.7 \%$ & $16.8 \%$ \\
\hline$D T S-L 2$ & $26.9 \%$ & $5.3 \%$ & DTS-L11 & $73.5 \%$ & $47.6 \%$ \\
\hline DTS-L12 & $32.2 \%$ & $5.5 \%$ & $b-17$ & $79.8 \%$ & $48.2 \%$ \\
\hline$b-9$ & 18.2 & $5.9 \%$ & & & \\
\hline
\end{tabular}

persists throughout development without a sharp lethal phase, resulting in gradually decreasing number of survivors. DTS/DTS homozygotes perish under permissive temperatures principally as late embryos, a minor amount of animals hatch and the emerging adults are extremely weak, and die prematurely (Suzuki and Procunier, 1969; Szidonya and Reuter, 1988; Szidonya et al., 1990). Adult DTS/+ heterozygotes are viable, but become gradually female-sterile under restrictive temperatures (Suzuki and Procunier, 1969).

Cell-matrix adhesions in Drosophila are known to involve, in addition to type IV collagen, all basic constituents of the basement membrane, including laminin, nidogen/entactin, perlecan, integrins and cross-linking lysyl oxidases (Hynes and Zhao, 2000; Molnar et al., 2005). Although the biochemistry and physiology of Drosophila type IV collagen $\alpha 1$ (IV) chain has been extensively studied (Blumberg et al., 1987, 1988; Fessler et al., 1993), only limited information is available on the genetics of this protein. Importantly, ectopic expression of internally deleted and antisense col4a1 has been previously noted to affect muscle attachment and gut morphology (Borchellini et al., 1996).

Collections of Drosophila, P-element-induced lethal mutations provide a robust tool to identify genetically interacting loci (Török et al., 1993; Deák et al., 1997). This approach has led to the discovery of the col4a2 gene (Yasothornsrikul et al., 1997), and demonstrated that both the functional col4a1 and col4a2 genes are necessary for viability (Rodriguez et al., 1996).

As a first step toward characterizing dominant temperaturesensitive alleles, we have analyzed the Drosophila l(2)25Ca locus using genetic, molecular and cell biology methods and provide evidence that the dominant temperature-sensitive mutations localize within the col4a1 gene. Based on ultrastructural, histology and behavioral data, we show that missense mutations within the coding region of the col4a1 gene result in a severe myopathic phenotype.

\section{Results}

\subsection{Complementation mapping of the l(2)25Ca locus}

The alleles of the $l(2) 25 \mathrm{Ca}$ locus include EMS-induced dominant heat- and cold-sensitive and recessive point mutations, $\mathrm{X}$-ray generated lesions, P-element insertions and P-element reversions. We crossed all known 34 mutants of this locus in a pair-wise fashion in all

Table 3

Rescue of dominant temperature-sensitivity by a col4a1 transgene integrated in the second or third chromosome in animals incubated at $29^{\circ} \mathrm{C}$. Survival rates are percentages of interchromosomal recombinants raised without DTS alleles in the same progeny. The number of the interchromosomal recombinants varied between 50 and 100 animals in individual experiments.

\begin{tabular}{lcll}
\hline col4a1 allele & Chr. 3 integration & Chr. 2 integration & Without transgene \\
\hline DTS-L3 & $14.8 \%$ & $29.7 \%$ & $1.6 \%$ \\
DTS-L2 & $13.4 \%$ & $26.5 \%$ & $2.9 \%$ \\
a-30 & $29 \%$ & $53.8 \%$ & $5.5 \%$ \\
\hline
\end{tabular}

Table 4

Rescue of col4a1 and col4a2 recessive lethal alleles by two copies of a col4a1 transgene. Alleles of col4a2 could not be complemented. Survival rates are percentages of the interchromosomal recombinants derived from 50 to 100 animals.

\begin{tabular}{llll}
\hline col4a1 allele & $\begin{array}{l}\text { Two transgenic } \\
\text { col4a1 copies }\end{array}$ & col4a2 allele & $\begin{array}{l}\text { Two transgenic } \\
\text { col4a1 copies }\end{array}$ \\
\hline$b-9 / b-29$ & $45 \%$ & $s z-14 / b-35$ & $0 \%$ \\
$b-9 / h-13$ & $62 \%$ & $s z-14 / b-14$ & $0 \%$ \\
& & $b-14 / b-35$ & $0 \%$ \\
\hline
\end{tabular}

combinations in 561 independent crosses (Table 1A, B). The data from these experiments enabled us to construct a complementation map that proved to be sharply divided into two non-overlapping parts. The results confirmed the presence of two closely linked genes within this chromosomal location (Table 1C). As one side of the map contained all the dominant temperature-sensitive alleles it is likely that only one of the two genes carries this type of mutation.

The cluster of $l(2) 25 \mathrm{Ca}$ alleles, containing the temperaturesensitive variants, performed intragenic trans complementation in various combinations at permissive temperature. Adult dominant/ dominant, dominant/recessive, and recessive/recessive allelic transheterozygotes showed low viability, remained stuck in the medium and died prematurely, whereas homozygotes perished early in development. The results of both inter- and intragenic complementation tests are summarized in a complementation matrix (Table $1 \mathrm{~A}$ ).

We have determined the survival rate for dominant temperaturesensitive animals reared at $29^{\circ} \mathrm{C}$. The number of adult offspring varied between $0.5 \%$ and $50 \%$ of those incubated at $20^{\circ} \mathrm{C}$. The results also showed allele-specific survival rate for the heat-sensitive DTS allelic series. Allele-specific survival was further supported by the ratio of DTS/+ pupae/adults that varied between 10 and 60\% (Table 2). These results were consistent with the semi-lethality observed in previous temperature-shift experiments with increasingly reduced number of survivors during all developmental phases (Szidonya et al., 1990).

\subsection{Molecular analysis of the l(2)25Ca locus}

In subsequent experiments we further analyzed the chromosomal location narrowed down by the dominant mutations. The $l(2)$ hsneo119 and $l(2)$ hsneo10 P-element insertions induced recessive lethal mutations that proved to be allelic with each other and with the DTS mutations that are clustered in one side of the complementation matrix (Table 1A). We have isolated genomic DNA from these mutants as an EcoRI fragment that flanked the transposon insertions. Using DNA sequencing we determined the insertional sites for $l(2)$ hsneo119 and $l(2) h s n e 010$ transposons and found these to be integrations of 145 and 36 nucleotides upstream from the transcriptional start site of the col4a1 gene. DNA sequence data were deposited in the EMBL database under the accession numbers AJ831378 and AJ831377. The nucleotide sequence on the opposite side of the genomic insert of the $l(2) h s n e 0119$ contained the EcoRI site and corresponded to exons 5 and 6 and intron 5 of the col4a1 gene. Southern blot analysis using genomic DNA from these mutants and the EcoRI genomic fragment as a probe confirmed heterozygosity of the insertional alleles $l(2) h s n e 0119$ and $l(2) h s n e o 10$. Alleles $h-13$ and $h-30$ carried deletions within the coding region of the col4a1 gene (data not shown). Our genetic and molecular data were consistent with the known position and head-to-head genomic organization the of the two Drosophila type IV collagen genes (Yasothornsrikul et al., 1997). The close linkage of the two loci was further supported by the lack of recombinants in various col4a1 and col4a2 crosses.

Northern blot analyses using embryonic RNA and the EcoRI genomic probe detected a $7 \mathrm{~kb}$ mRNA band. Wholemount in situ hybridization in late embryos confirmed expression of the col4a1 gene in haemocytes (data not shown) as reported previously (Monson et al., 1982; Fessler and Fessler, 1989). We have isolated the genomic 


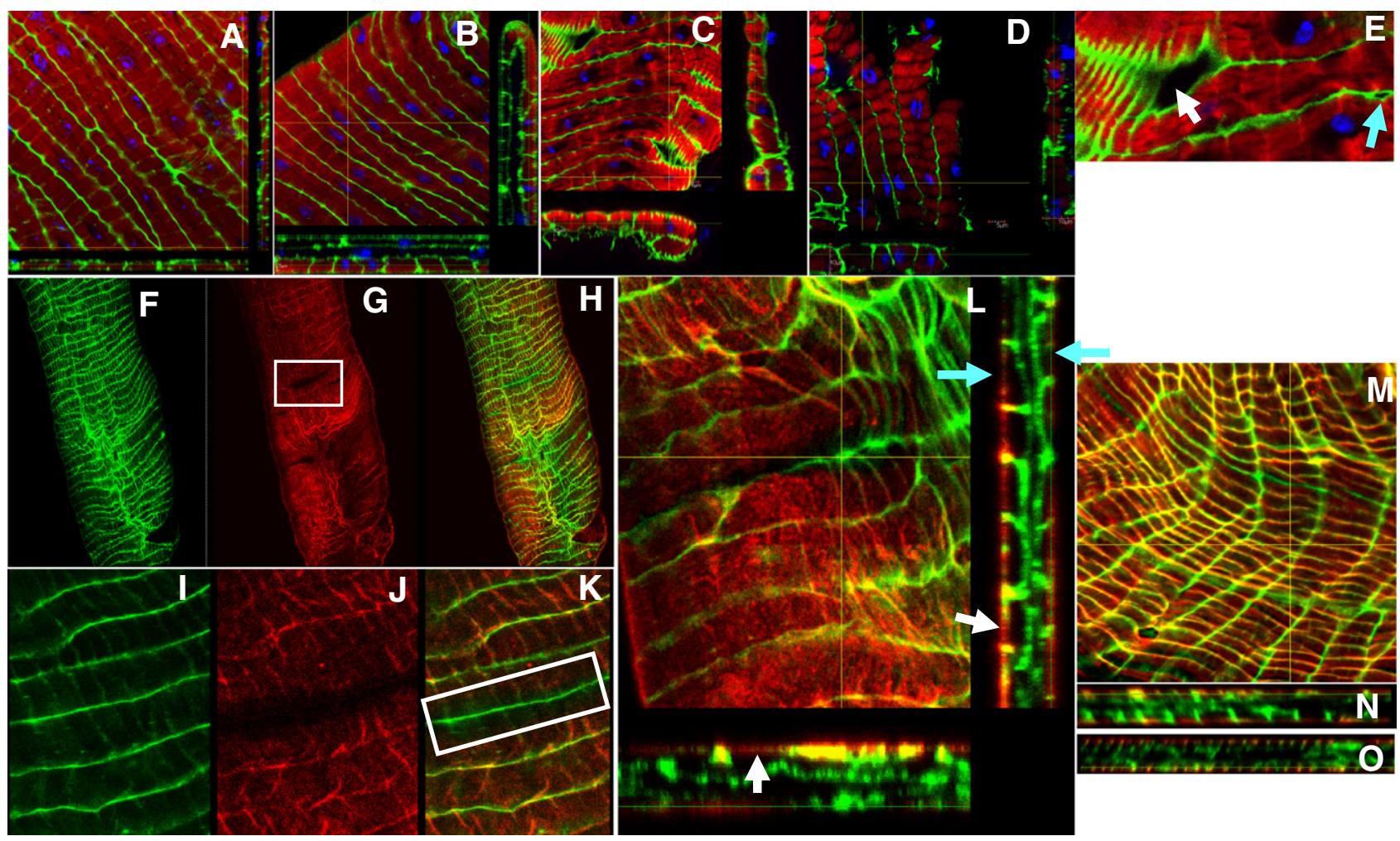

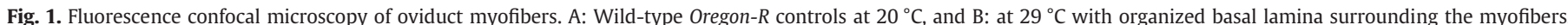

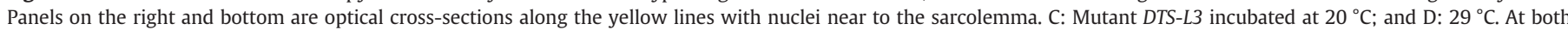

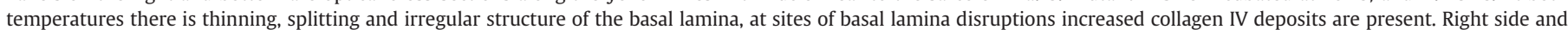

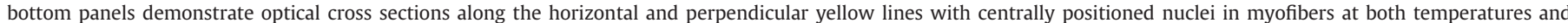

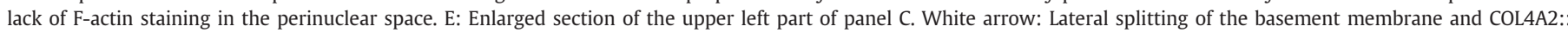

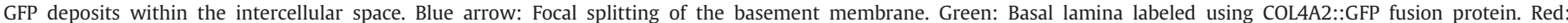

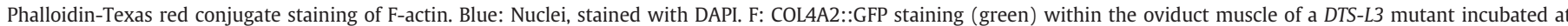

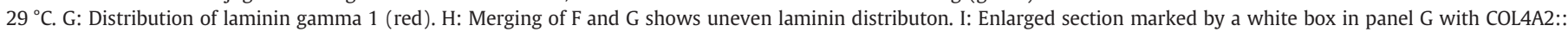

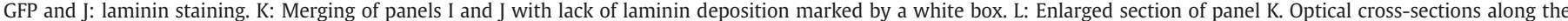

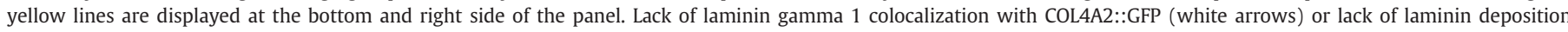

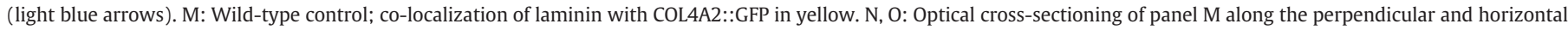
yellow lines; laminin is co-localized with COL4A2::GFP.

fragment containing the entire col4a1 gene from a cosmid library and subcloned it as a $9.7 \mathrm{kbp} \mathrm{Stul} \mathrm{fragment} \mathrm{in} \mathrm{the} p P\{C a S p e R-h s\}$ vector under the control of the Hsp70 promoter. Using this construct, transgenic lines were generated with insertions in the second and third chromosomes in DTS-L3, DTS-L2 and $a-30$ animals. The col4a1 transgene proved to be sufficient for a partial rescue of the dominant temperature sensitivity in all three mutants (Table 3). However, the transgene in a single copy did not complement the recessive lethality of col4a1 compound heterozygotes. For such rescue two copies of the transgene were required (Table 4). Mutant alleles of the col4a2 gene were not rescued by either one or two transgenic copies of the col4a1 gene. These results demonstrated that the DTS mutant phenotype is caused by lesions within the col4a1 gene.

\subsection{Oviductal muscle phenotype of col4a1 mutant DTS-L3}

The Drosophila female reproductive tract includes the ovarioles, ovaries, lateral and common oviducts and the uterus. The muscle fibers of the tract form a single layer and are striated with a clear sarcomeric structure and provide the necessary mechanical force during egg maturation and deposition (Middleton et al., 2006).

Adult DTS/+ female heterozygotes failed to lay eggs and became increasingly sterile at $29^{\circ} \mathrm{C}$ consistent with the observation that in myopathic conditions the muscle develops normally, but degenerates following repeated exposure to stress (Sanes, 2003). This gradual manifestation of the phenotype, that indicated muscle defects, prompted us to investigate the structure of the myofiber layer of oviducts in the severely affected $D T S-L$ (ethal)3 mutant animals using COL4A2::GFP label and fluorescence confocal microscopy. Oviductal myofibers in control Oregon- $R$ COL4A2::GFP flies showed regular shape and striation, peripheral nuclei and tight sarcolemma-basement membrane association at both temperatures (Fig. 1A, B). Muscle fibers in DTS-L3 mutant detached laterally from each other with focal splitting of the basement membrane (Fig. 1C, D, E). Between detached muscle fibers altered and thickened collagen IV-associated label appeared to fill the gaps (Fig. 1E). Myofibers were partially rounded up at their otherwise spiky ends, the nuclei were centrally located, and within the perinuclear space, phalloidin staining for F-actin was diminished or missing even at permissive temperature (Fig. 1C, E). At restrictive temperature, loss of F-actin staining extended to large areas of the sarcoplasm, myofibers with loose lateral contact were prominently rounded, and their nuclei were central with condensed chromatin. The basement membrane surrounding the myofibers appeared distorted, thinned, or absent (Fig. 1D). These results demonstrated that disruption of the COL4A1 protein induced a severe oviductal muscle phenotype and caused a gradually developing female sterility in mutant animals.

Laminin gamma 1 immunostaining in the oviduct of DTS-L3 mutants incubated at $20^{\circ} \mathrm{C}$ showed alterations that became more pronounced at $29^{\circ} \mathrm{C}$ (Fig. 1L) and included absence of colocalization with collagen IV, and missing laminin staining particularly at sites where myofibers separated from each other (Fig. 1F-K). While in controls laminin staining was regular and fully overlapped with collagen IV staining (Fig. 1M-O). 

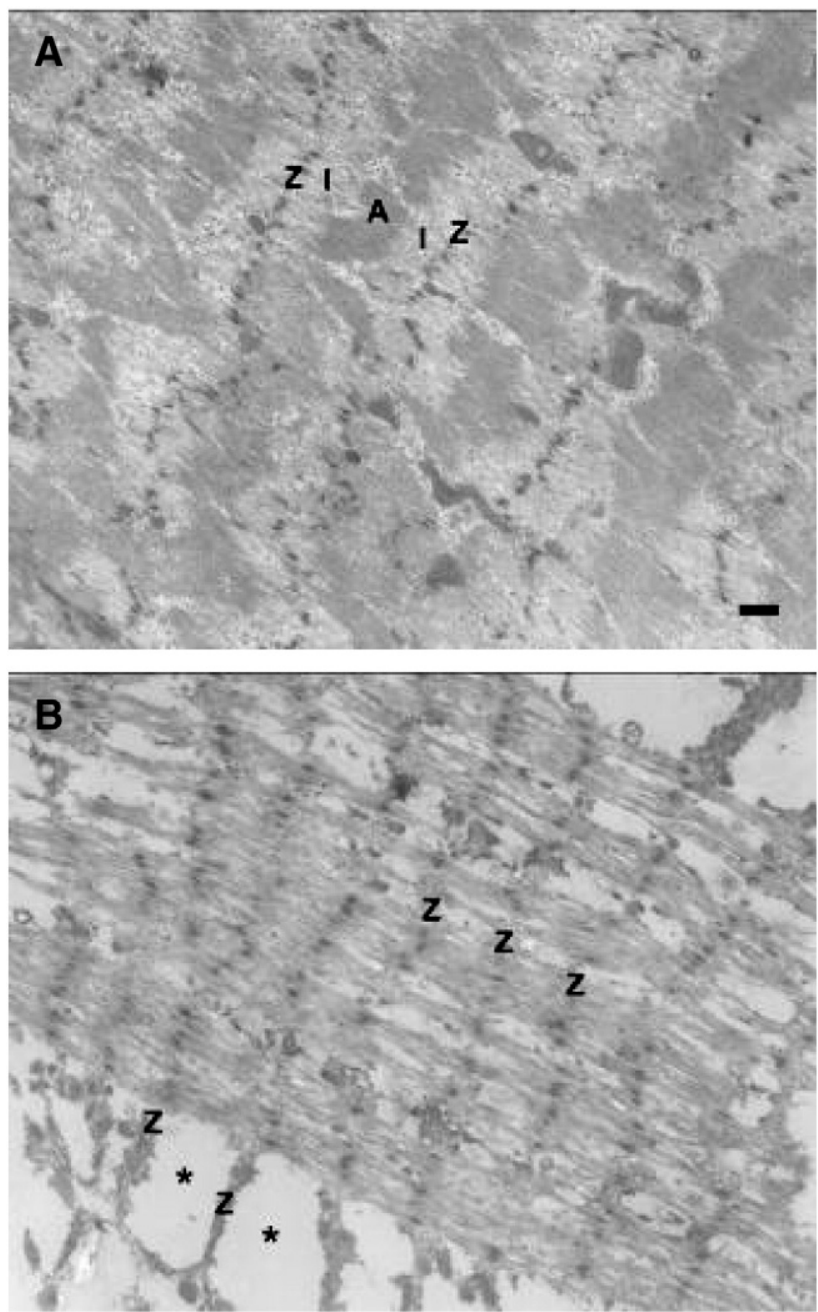

Fig. 2. The ultrastructure of striated body wall muscles of $D T S-L 3 /+\mathrm{L} 3$ stage larvae. A: At $20^{\circ} \mathrm{C}$ sarcomeres with precise transverse alignment and comprised dense $\mathrm{Z}$ and $\mathrm{A}$ bands and wide I bands. B: At $29^{\circ} \mathrm{C}$ the $\mathrm{A}$ and I bands are not distinguished, prominent degeneration of muscle fibers appear as electronlucent areas $\left({ }^{*}\right)$ between persisting $\mathrm{Z}$ bands. Bar equals $1 \mu \mathrm{m}$.

\subsection{Ultrastructural skeletal muscle changes in DTS-L3 mutants}

A prominent phenotypic feature of freshly hatched larvae of the DTS-L3 heterozygous mutant that carry the most deleterious allele within this series of mutants, raised at $29^{\circ} \mathrm{C}$, is that they stop active crawling and eager pursuit of food at larval stage 3 (L3) suggesting loss of coordinated action of muscle fibers. Electron microscopic analysis of longitudinal ultrathin sections of striated muscles of the body wall of L3 larvae even at permissive temperature showed degenerative changes. Sarcomeres of the muscles lacked precise transverse alignment and comprised dense $\mathrm{Z}$ and $\mathrm{A}$ bands and wide I bands (Fig. 2A). At restrictive temperature, only $Z$ bands could be recognized, the $A$ and $I$ bands were not separated and degradation of muscle fibers appeared as electron-lucent areas between persisting $\mathrm{Z}$ bands (Fig. 2B). These results correlated with the lack of actin staining we noted in sarcomeres within large areas of oviductal myofibers in animals raised at $29^{\circ} \mathrm{C}$ and confirmed myopathy in a functionally different muscle type.

\subsection{Decreased COLAA1 protein in DTS-L3 mutants at $29^{\circ} \mathrm{C}$}

For Western blot analysis protein extracts isolated from Oregon- $R$ and DTS-L3 3rd instar (L3) larvae and adults, incubated both at $20{ }^{\circ} \mathrm{C}$ and $29^{\circ} \mathrm{C}$, were blotted and immunostained using Drosophila

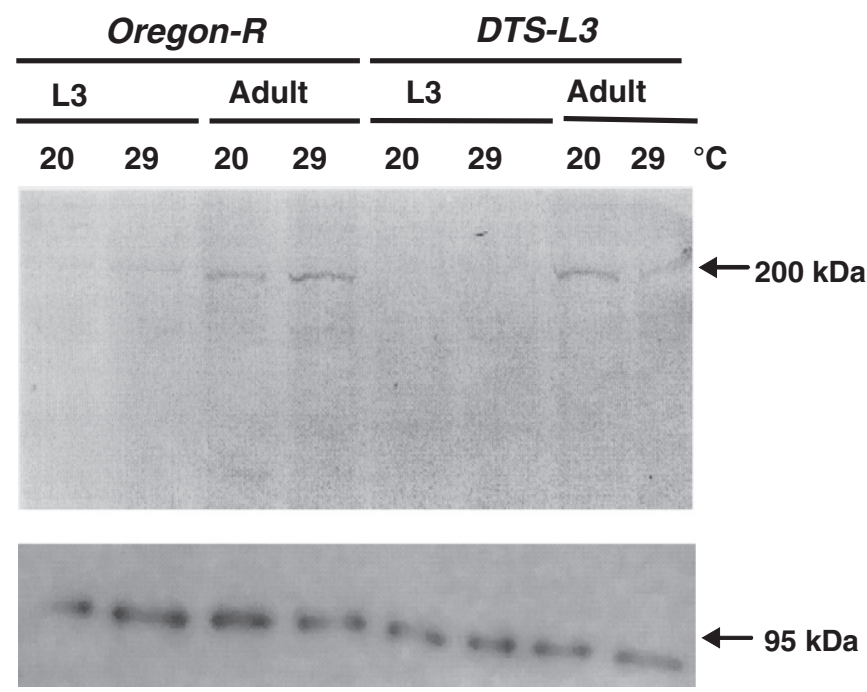

Fig. 3. Upper panel: Western blot analysis of COL4A1 in Oregon- $R$ and DTS-L3 mutant protein extracts prepared from larva (L) and adult animals incubated at $20^{\circ} \mathrm{C}$ and $29^{\circ} \mathrm{C}$ using Drosophila polyclonal anti-collagen IVa1 antibody. The $200 \mathrm{kD}$ COL4A1 band is expressed at low levels in larvae, it is present in normal adult controls, but in the mutant it is not detectable in larval extracts. In adult mutants it is present at $20^{\circ} \mathrm{C}$, but diminished at $29^{\circ} \mathrm{C}$. Lower panel: Protein expression control alpha glycogen phosphorylase.

polyclonal anti-collagen IVa1 antibody. A single band of about $200 \mathrm{kDa}$ was detected consistent with the previously reported size of the COL4A1 monomer (Fessler et al., 1993). In samples from wild-type larvae a faint band was present at both temperatures, while in mutant larvae it was not detected. In wild-type adults the $200 \mathrm{kDa}$ band was present at both temperatures and also in the mutant $20{ }^{\circ} \mathrm{C}$ samples, but with reduced intensity in $29^{\circ} \mathrm{C}$ samples (Fig. 3). For protein expression control alpha-glycogen protein was used.

In subsequent analysis of the expression levels of the extracellular matrix and matrix-associated genes in adult DTS-L3 mutant samples exposed to $29^{\circ} \mathrm{C}$, the level of the col4a1 mRNA was found actually slightly increased ( 1.21 fold) indicating that decreased COL4A1 protein level was not due to transcriptional changes. Collectively these results, further supported by decreased deposition observed using GFP label in the mutant oviduct at $29{ }^{\circ} \mathrm{C}$ (Fig. 1C, D), suggest that the decreased level of COL4A1 protein at $29^{\circ} \mathrm{C}$ in this mutant likely results from decreased assembly and deposition.

2.6. DTS-L3 animals carry four missense and a noncoding col4a1 gene mutations

Exonic sequences of the col4a1 gene were amplified from genomic DNA template isolated from adult DTS-L3/+ animals. We identified four missense mutations within the coding region and a single nucleotide polymorphism in the 3' UTR of the col4a1 gene (Fig. 4A-E). Two of the coding sequence mutations proved to be transitions. G1882A caused a glycine to asparagine substitution (G552D) and G3468A induced an alanine to threonine change (A1081T). The two other mutations were transversions, G3602T a lysine to asparagine substitution (K1125N), and G3820C a glycine to alanine substitution (G1198A). Nucleotide numbers were based on the reference sequence NM_164615. The fifth mutation was a G to A transition within the 3' UTR region of the col4a1 gene. The positions of these mutations are displayed on the mRNA map of the col4a1 gene (Fig. 4F). Both the A1081T and K1125N substitutions affect the Y within the Gly-X-Y unit. Additionally, the other two mutations, G552D and G1198A, affect glycine codons that are considered to be deleterious mutations of 
A

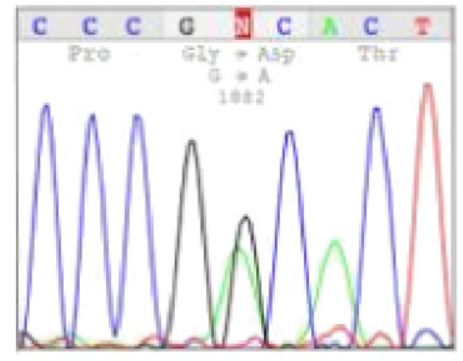

B

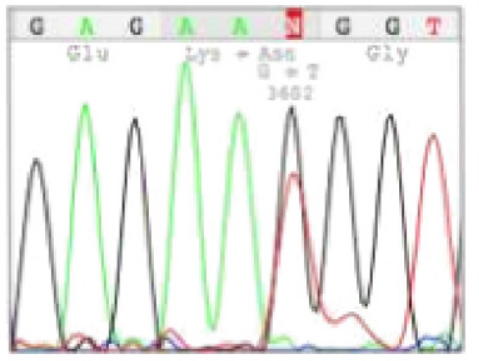

E

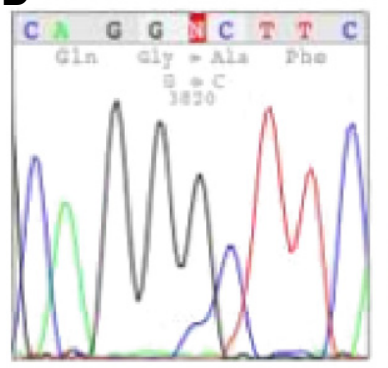

E

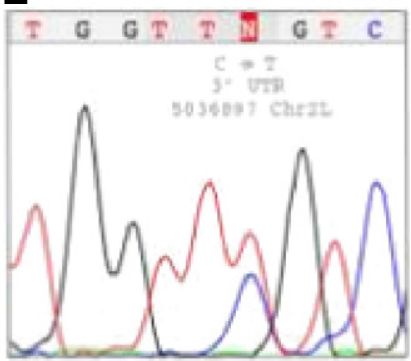

C

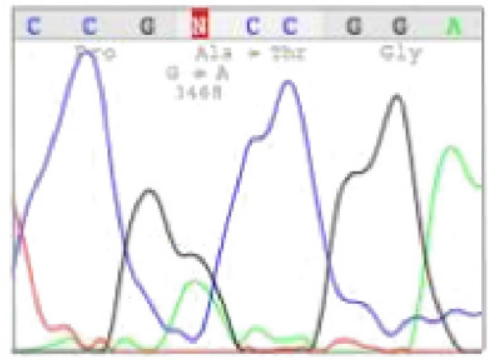

F

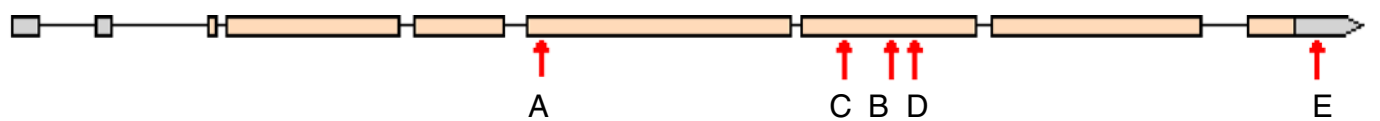

G

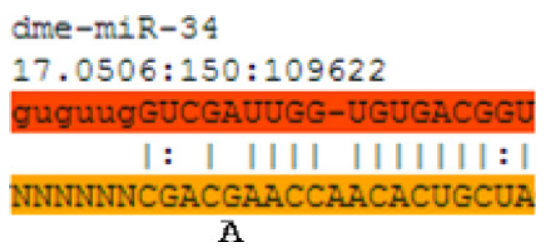

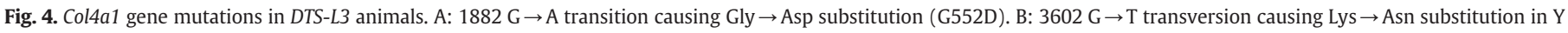

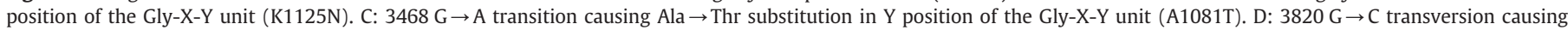

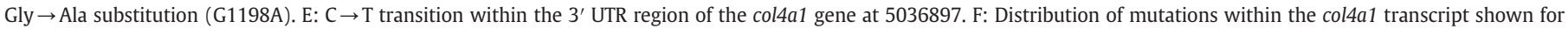

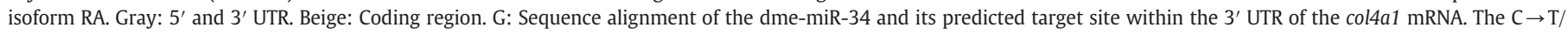
$\mathrm{G} \rightarrow \mathrm{A}$ transition does not appear to influence the pairing of the micro RNA.

collagens. The G to A transition in the $3^{\prime}$ UTR region is localized within the micro-RNA binding site of dme-miR-34 (Sempere et al., 2003), although the mutation does not appear to affect the putative base pairing of dme-miR-34 (Fig. 4G).

\section{Discussion}

Our intergenic complementation data generated by using thirtyfour mutant alleles of the col4a1 and col4a2 genes confirmed an initial report of Rodriguez et al. (1996) that both type IV collagen genes are needed for viability and their gene products are indispensable in Drosophila. In our experiments, all alleles of the col4a2 gene performed full trans complementation, except transposon insertions l(2)hsneo80 and $v \mathrm{~kg}^{\mathrm{SAK}}$ (Yasothornsrikul et al., 1997) that provided partial complementation of the col4a1 alleles. The $l(2) h s n e 0119$ and $l(2) h s n e 010$ P-element insertions complemented all col4a2 alleles and also performed interallelic complementation with some of the col4a1 alleles. These results indirectly indicated a shared promoter near to the col4a2 gene, and explained the observed hypomorph nature of the col4a2 $5^{\prime}$ insertions. The two sharply separated regions of our intergenic complementation matrix confirmed the previously reported, conserved head- to-head organization and shared promoter elements of the two type IV collagen genes (Yasothornsrikul et al., 1997; Kashtan, 1999).

A characteristic feature of an antimorph, dominant-negative mutation is that the phenotype is partially complemented by an increasing dosage of the wild-type gene, as the dominant-negative effect is triggered during incorporation of mutant polypeptides that results in a more severe phenotype than those of null heterozygotes (Bruckner-Tuderman and Bruckner, 1998). Indeed, our data suggested antimorph nature for both dominant and recessive phenotypes of the col4a1 mutations that were partially complemented at different levels by the col4a1 transgene depending on the copy number and the site of integration. Furthermore, exactly the same transgenic dosage rescued the $b-9 / h-13$ mutant/null compound heterozygote at a higher efficiency than the mutant/mutant compound heterozygote. These data clearly showed that the DTS mutations affected the col4a1 gene.

Two mutant alleles of the same gene may, in some instances, restore the wild-type function in interallelic complementation and support biochemical conclusions (Clifford and Schüpbach, 1994). In principle, the two type IV collagen loci in Drosophila allow the homotrimer $[\mathrm{COL} 4 \mathrm{~A} 1]_{3}, \quad[\mathrm{COL} 4 \mathrm{~A} 2]_{3}$ or heterotrimer $[\mathrm{COL} 4 \mathrm{~A} 1]_{2} \mathrm{COL} 4 \mathrm{~A} 2, \mathrm{COL} 4 \mathrm{~A} 1$ $[\mathrm{COL} 4 \mathrm{~A} 2]_{2}$ compositions of the triple helical protomers. Homozygotes of 
both col4a1 and col4a2 mutations are recessive lethal, therefore, exclude homotrimers as a possible composition. This hypothesis is further supported by the fact that triple helices of multiple mammalian type IV collagens in different tissues are always heterotrimers (Boutaud et al., 2000).

We observed interallelic complementation predominantly among the temperature-sensitive col4a1 alleles demonstrating that two mutant proteins, in certain combinations, may provide viability in compound heterozygotes. This observation indirectly suggests that two COL4A1 chains interact in forming the trimer. Regardless of the nature of the mutant col4a1 alleles, in combination with the wild-type allele no recessive lethality arose, the progeny was viable and fertile in our experiments. This result was also supported by viable crosses carrying the deletion null alleles $h-13$ and $h-30$, indicating that a single wild-type copy was necessary and sufficient for viability in null/+ heterozygotes. On the other hand, neither mutant col4a1 allele complemented the $h-13$ and $h$-30 deletions, demonstrating the need of COL4A1 chains that are able to assemble with each other, therefore Drosophila basement membrane likely has a [COL4A1 $]_{2}$ COL4A2 protomer composition.

Col4a1/2 $2^{-1-}$ mice null mutants die in early embryonic development (Pöschl et al., 2004). Caenorhabditis elegans with recessive null, or with semi-dominant temperature-sensitive alleles, also die during embryogenesis with intracellular accumulation of the mutant a1(IV) and a2 (IV) chains, indicating defective secretion and/or assembly of these proteins (Gupta et al., 1997). Type IV collagen mutations described in certain types of human Alport syndrome similarly result in the synthesis of mutant collagen chains, likely without correct assembly of triple helices and subsequent incorporation into the basement membrane (Kashtan, 2000). In contrast, in Drosophila, semi-lethality of DTS/+ heterozygotes at $29^{\circ} \mathrm{C}$ during development, and the lack of a sharp lethal phase suggest some degree of assembly of triple helices with integration of the mutant chains and deposition into the basement membrane resulting in sub-vital conditions and demonstrate somewhat different features of Drosophila basement membrane collagens.

Analysis of the mutational sites in DTS-L3 animals revealed four missense mutations within the coding region of the col4a1 gene. The DTS-L3 mutant was induced by EMS, which preferentially alkylates the guanine base, and the product, $\mathrm{O}$-6-ethylguanosine mispairs with $\mathrm{T}$ in the next replication, causing a $\mathrm{G} / \mathrm{C}$ to $\mathrm{A} / \mathrm{T}$ transition, that was observed as G552D and A1081T amino acid exchanges in the coding region, and a $G$ to $A$ transition in the $3^{\prime}$ UTR. The two transversion events (K1125N, G1198A) are likely spontaneous mutations arising during DNA replication.

Given the essential function of the basement membrane in most organs and tissues and the pleiotropic phenotype of the mutants, Drosophila col4a1 mutations closely resemble the effect of mammalian COL4A1 and COL4A2 mutations including myopathy, that in Col4a1 ${ }^{ \pm} / \Delta x 40$ mice manifested in split muscle fibers and nonperipheral nuclei (Labelle-Dumais et al., 2011). The appearance of central nuclei is a general feature in various myopathies and muscular dystrophies during cycles of degeneration and regeneration of human and mouse muscle fibers. Mutations in human genes myotubularin (Jeannet et al., 2004; Dowling et al., 2009), myotubularin-related protein 14 (Tosch et al., 2006), amphiphysin (Nicot et al., 2007), and dynamin 2 (Bitoun et al., 2005) similarly associate with centronuclear myopathies. Drosophila mutants for myotubularin (CG9115; Construct ID: 14121), amphiphysin (Leventis et al., 2001; Razzaq et al., 2001), and dynamin (Masur et al., 1990) have been also reported with various degrees of muscle involvement, however, without central nuclei in myofibers. Therefore, our data with col4a1 mutant oviductal myofibers represents the first demonstration of central nucleopathy in Drosophila. The morphological changes of myofibers that we observed also resemble the progressive muscular dystrophy reported for the major laminin-binding integrin alpha7-deficient mice (Mayer et al., 1997).

In Col4a1 $1^{ \pm / \Delta e x 40}$ mice, anti-laminin antibody staining revealed focal disruption or absence of the hyaloid vasculature from the inner limiting membrane in transverse eye sections (Labelle-Dumais et al., 2011). In mouse Col4a1 ${ }^{ \pm / R a w}$ mutants, continuous laminin staining was reported in the descending aortae, however, closer inspection of the images revealed scattered and torturous laminin deposition with markedly uneven intensities (van Agtmael et al., 2010). Of these laminin features we noted similarly less intensive, uneven, or focally absent immunostaining in oviducts of Drosophila col4a1 mutants.

Several clinical genetic studies reported patients with COL4A1 mutations with basement membrane defects and pathological manifestation in multiple tissues. Muscle weakness, cramps, high creatine kinase levels (Plaisier et al., 2007), bilateral leg muscle contractures (Gould et al., 2005, 2006) and dystrophy and atrophy of leg muscles (Breedveld et al., 2006) have been noted, strongly supporting muscle manifestation of COL4A1 mutations. However, in-depth histology, cell-based and biochemical studies of the mutant muscle tissue are lacking and significant details of the underlying pathomechanism remain unknown. Therefore, our Drosophila col4a1 mutants may serve as effective models to uncover the mechanisms by which COL4A1 mutations result in compromised myofiberbasement membrane interactions and manifest in aberrant muscle function in this emerging COL4A1-associated disorder.

\section{Experimental procedures}

\subsection{Mutant strain maintenance and generation of col4a2::GFP heterozygotes}

Stocks were maintained at $20^{\circ} \mathrm{C}$ or $29^{\circ} \mathrm{C}$ on yeast-cornmeal-agarsucrose medium containing propionic acid and hydroxymethylbenzoate to avoid fungal infections. The mutant stocks were kept heterozygous over the CyRoi balancer chromosome. Screening of allelic P-insertions was carried out as described (Török et al., 1993; Deák et al., 1997). Survival rates were determined by incubating 200-300 0-8 h embryos at $20^{\circ} \mathrm{C}$ and $29^{\circ} \mathrm{C}$ in three subsequent experiments. The basement membrane was visualized using a Viking-GFP protein fusion (GFP in the protein trap with the COL4A2 from the viking gene) as molecular marker (Glise et al., 1995) and by selecting col4a1- col4a2/ + col4a2::GFP heterozygotes by balancer loss.

\subsection{Genetic complementation}

Strains (Table 1) were crossed in all pair-wise combinations and intergenic complementation was established at above 50\% progeny of the expected values in col4a1 $^{-}$col4a2/col4a1 col4a2- diploids. The threshold level of intragenic complementation in $\operatorname{col} 4 a 1^{\mathrm{X}} /$ col4a $1^{Y}$ heteroallelic combinations was $30 \%$ of the expected progeny. Partial complementation occurred under the threshold levels. Both threshold levels were established by the loss of the balancer chromosome, CyRoi. This balancer chromosome carries two dominant mutations causing curly wings and rough eyes and its intrachromosomal inversion prevents recombination of the non-sister chromatids.

\subsection{Nucleic acid analysis}

Genomic DNA extraction and plasmid rescue from P-elementbearing strains were performed as described previously (Burmester et al., 2000). Electrophoresis and blotting of nucleic acids, RNA purification and polyA $^{+}$selection, non-radioactive- or radiolabelling, Southern and Northern analyses, PCR amplification of col4a1 exon sequences and DNA sequencing were carried out according to standard methods (Sambrook et al., 1989). BLAST programs developed for homology searches at nucleotide or amino acid levels were applied (Altschul et al., 1990). In situ hybridization to late (18-24 h) Drosophila wholemount embryos was done as described by Komonyi et al. (1998). MicroRNA binding sites were predicted by Microcosm (http://www.ebi.ac.uk/enright-srv/microcosm/htdocs/targets/v5/). 


\subsection{Analysis of the oviducts}

Oviducts were removed under carbon dioxide anesthesia from female adults that were grown at permissive temperature and kept for 10 days at $29^{\circ} \mathrm{C}$. Dissected oviducts were fixed in $3.7 \%$ formaldehyde dissolved in phosphate buffered saline (PBS) for $10 \mathrm{~min}$, washed three times with PBS, permeabilized in $0.1 \%$ Triton $X$ dissolved in PBS, washed in PBS and stained with 1 unit Phalloidin-Texas red (Invitrogen), dissolved in $0.2 \mathrm{ml}$ PBS, and the nuclei with $10 \mu \mathrm{g} / \mathrm{ml}$ DAPI. Optical sectioning of the oviducts was achieved by confocal fluorescence microscopy (Olympus FV 1000). Rabbit polyclonal antibody to Drosophila laminin gamma 1 (Abcam) was used for immunohistochemistry to label basement membrane laminin that was detected by anti-rabbit secondary antibody conjugated by Alexa 564 (red).

\subsection{Western analysis}

Isolation of polypeptides from larvae and adults, followed by immunostaining, was performed according to Fessler et al. (1993). Concentrations of protein solutions isolated was measured according to Bradford (1976) and $10 \mu \mathrm{g}$ of proteins was loaded in each lane. The Drosophila polyclonal anti-collagen type IV a1 antibody (bleed IV, rabbit), recognizing both the $\mathrm{N}$ - and C-termini of the Drosophila COL4A1 protein was a generous gift of Prof. J.H. Fessler (UCLA, USA). Antirabbit mouse monoclonal antibody (Sigma) conjugated with peroxidase served as secondary antibody. As a protein expression control, alpha-glycogen phosphorylase was detected by polyclonal rabbit antibody; anti-rabbit antibody, conjugated with peroxidase, allowed detection of the signal.

\subsection{Transmission electron microscopy}

Body wall muscles from freshly hatched L3 stage DTS-L3/+ animals were dissected and fixed in $0.1 \mathrm{~N} \mathrm{pH} 7.4 \mathrm{Na}$-cacodylate (Sigma) buffer containing $3.2 \%$ paraformaldehyde (TAAB), $0.5 \%$ glutaraldehyde (TAAB), $1 \%$ sucrose (Sigma) and $40 \mathrm{mM} \mathrm{CaCl}_{2}$ (Reanal) overnight at $4{ }^{\circ} \mathrm{C}$ and embedded in Araldyte (Fluka). Ultrathin sections $(80 \mathrm{~nm})$ were post-contrasted by immersing the grids in lead citrate (Reanal). Images were taken in a JEOL CM-II 100 transmission electron microscope. Samples from three different animals were evaluated.

\subsection{Microarray experiment}

RNA was extracted from 24 DTS-L3/+ heterozygous flies (12 females, 12 males). $24 \mathrm{~h}$ following their emerging from the pupae, the control and heat-shocked flies were kept either at 18 or $29^{\circ} \mathrm{C}$ for 3 days. Trizol reagent (Invitrogen) was used according to the manufacturer's recommendations. We eliminated the DNA contamination of the samples by the Ambion DNA-free kit (\#AM1906), followed by RNeasy mini kit (Qiagen) chromatography to obtain high purity RNA. The labeling of RNA was carried out by two-color Quick-Amp labeling kit (Agilent) and hybridized to Agilent 4x44k Drosophila specific array (Design ID: 18972). Feature extraction was achieved by an Agilent Technologies Scanner G2505B. Data analysis was done by Agilent GeneSpring GX version 11.0.

\section{References}

Alport, A.C., 1927. Hereditary familial congenital haemorrhagic nephritis. Br. Med. J. 1, 504-506.

Altschul, S.F., Gish, W., Miller, W., Myers, E.W., Lipman, D.J., 1990. Basic local alignment search tool. J. Mol. Biol. 215 (3), 403-410.

Van Agtmael, T., Schlötzer-Schrehardt, U., McKie, L.G., Brownstein, D.W., Lee, A., Cross, S.H., Sado, Y., Mullins, J.J., Pöschl, E., Ian, J., Jackson, I.J., 2005. Dominant mutations of Col4a1 result in basement membrane defects which lead to anterior segment dysgenesis and glomerulopathy. Hum. Mol. Genet. 14, 3161-3168.
Van Agtmael, T., Bailey, M.A., Schlötzer-Schrehardt, U., Craigie, E., Jackson, I.J., Brownstein, D.G., Megson, I.L., Mullins, J.J., 2010. Col4a1 mutation in mice causes defects in vascular function and low blood pressure associated with reduced red blood cell volume. Hum. Mol. Genet. 19, 1119-1128.

Bitoun, M., Maugenre, S., Jeannet, P.-Y., Lacene, E., Ferrer, X., Laforet, P., Martin, J.-J. Laporte, J., Lochmuller, H., Beggs, A.H., Fardeau, M., Eymard, B., Romero, N.B., Guicheney, P., 2005. Mutations in dynamin 2 cause dominant centronuclear myopathy. Nat. Genet. 37, 1207-1209.

Blumberg, B., McKrell, A.J., Olson, P.F., Kurkinen, M., Monson, J.M., Natzle, J.E., Fessler, J.H., 1987. Basement membrane collagen IV and its specialized carboxyl domain are conserved in Drosophila, mouse and human. J. Biol. Chem. 262, 5947-5950.

Blumberg, B., McKrell, A.J., Fessler, J.H., 1988. Drosophila basement membrane collagen a1(IV). J. Biol. Chem. 263, 18328-18337.

Borchellini, C., Coulon, J., Le Parco, Y., 1996. The function of type IV collagen during Drosophila muscle development. Mech. Dev. 58, 179-191.

Boutaud, A., Borza, D.-B., Bondar, O., Gunwar, S., Netzer, K.-O., Singh, N., Ninomiya, Y., Sado, Y., Noelken, M.E., Hudson, B.G., 2000. Type IV collagen of the glomerular basement membrane. J. Biol. Chem. 275, 30716-30724.

Bradford, M., 1976. A rapid method for the quantitation of microgramm quantities of protein utilizing the principle of protein-dye binding. Anal. Biochem. 72, 248-254.

Breedveld, G., de Coo, I.F., Lequin, M.H., Arts, W.F.M., Heutink, P., Gould, D.B., John, S.W.M., Oostra, B., Mancini, G.M.S., 2006. Novel mutations in three families confirm a major role of COL4A1 in hereditary porencephaly. J. Med. Genet. 43, 490-495.

Bruckner-Tuderman, L., Bruckner, P., 1998. Genetic diseases of the extracellular matrix: more than just connective tissue disorders. J. Mol. Med. 76 (3-4), 226-237.

Burmester, Th., Mink, M., Pál, M., Lászlóffy, Zs, Lepesant, J.-A., Maróy, P., 2000. A genetic and molecular analysis in the 70CD region of the third chromosome of Drosophila melanogaster. Gene 246, 157-167.

Clifford, R., Schüpbach, T., 1994. Molecular analysis of the drosophila EGF receptor homolog reveals that several genetically defined classes of alleles cluster in subdomains of the receptor protein. Genetics 137, 531-550.

Deák, P., Omar, M.M., Saunders, R.D.C., Pál, M., Komonyi, O., Szidonya, J., Maróy, P. Zhang, Y., Ashburner, M., Benos, P., Savakis, C., Siden-Kiamos, I., Louis, C., Bolshakov, V., Kafatos, N., Madueno, F.C., Modolell, E., Glover, D.M., 1997. P-element insertion alleles of essential genes on the third chromosome of Drosophila melanogaster. Genetics 147, 1697-1722.

Dowling, J.J., Vreede, A.P., Low, S.E., Gibbs, E.M., Kuwada, J.Y., Bonnemann, C.G., Feldman, E.L., 2009. Loss of myotubularin function results in T-tubule disorganization in zebrafish and human myotubular myopathy. PLoS Genet. 5, e1000372.

Favor, J., Gloeckner, Ch.J., Janik, D., Klempt, M., Neuhäuser-Klaus, A., Pretsch, W. Schmahl, W., Quintanilla-Fend, L., 2007. Type IV procollagen missense mutations associated with defects of the eye, vascular stability, the brain, kidney function and embryonic or postnatal viability in the mouse, mus musculus: an extension of the Col4a1 allelic series and the identification of the first two Col4a2 mutant alleles. Genetics 175, 725-736.

Fessler, J.H., Fessler, L.I., 1989. Drosophila extrarcellular matrix. Annu. Rev. Cell Biol. 5, 309-339.

Fessler, L.I., Condic, M.L., Nelson, R.E., Fessler, J.H., Friström, J.W., 1993. Site-specific cleavage of basement membrane collagen IV during Drosophila metamorphosis. Development 117, 1061-1069.

Glise, B., Bourbon, H., Noselli, S., 1995. hemipterous encodes a novel Drosophila MAP kinase kinase, required for epithelial cell sheet movement. Cell 83, 451-461.

Gould, D.B., Phalan, F.C., Breedveld, G.J., van Mil, S.E., Smith, R.S., Schimenti, J.C., Aguglia, U., van der Knaap, M.S., Heutink, P., John, S.W.M., 2005. Mutations in Col4a1 cause perinatal cerebral hemorrhage and porencephaly. Science 308, 1167-1171.

Gould, D.B.F., Phalan, F.C., van Mil, S.E., Sundberg, J.P., Vahedi, K., Massin, P., Bousser, M.G., Heutink, P., Miner, J.H., Tournier-Lasserve, E., John, S.W.M., 2006. Role of COL4A1 in small-vessel disease and hemorrhagic stroke. N. Engl. J. Med. 354, 1489-1496.

Gupta, M.C., Graham, P.L., Kramer, J.M., 1997. Characterization of 1(IV) collagen mutations in Caenorhabditis elegans and the effects of $\alpha 1$ and $\alpha 2$ (IV) mutations on type IV collagen distribution. J. Cell Biol. 137, 1185-1196.

Hynes, R.O., Zhao, O., 2000. The evolution of cell adhesion. J. Cell Biol. 150, 89-96.

Jeannet, P.-Y., Bassez, G., Eymard, B., Laforêt, P., Urtizberea, J.A., Rouche, A., Guicheney, P., Fardeau, M., Romero, N.B., 2004. Clinical and histologic findings in autosomal centronuclear myopathy. Neurology 62, 1484-1490.

Kashtan, C.E., 1999. Alport syndrome. Medicine 78, 338-360.

Kashtan, C.E., 2000. Alport syndromes: phenotypic heterogeneity of progressive hereditary nephritis. Pediatr. Nephrol. 14 (6), 502-512.

Komonyi, O., Mink, M., Csiha, J., Maróy, P., 1998. Genomic organization of DHR38 gene in Drosophila: presence of Alu-like repeat in translated exon and expression during embryonic development. Arch. Insect Biochem. Physiol. 38, 185-192.

Labelle-Dumais, C., Dilworth, D.J., Harrington, E.P., de Leau, M., Lyons, D., Kabaeva, Zh. Manzini, M.C., Dobyns, W.B., Walsh, Ch.A., Michele, D.E., Gould, D.B., 2011. COL4A1 mutations cause ocular dysgenesis, neuronal localization defects, and myopathy in mice and Walker-Warburg syndrome in humans. PLoS Genet. 7 (5), e1002062.

Leventis, P.A., Chow, B.M., Stewart, B.A., Iyengar, B., Campos, A.R., Boulianne, G.L., 2001 Drosophila Amphiphysin is a post-synaptic protein required for normal locomotion but not endocytosis. Traffic 2 (11), 839-850.

Masur, S.K., Kim, Y.T., Wu, C.F., 1990. Reversible inhibition of endocytosis in cultured neurons from the Drosophila temperature-sensitive mutant shibirets1. J. Neurogenet. 6 (3), 191-206.

Mayer, U., Saher, G., Fässler, R., Bornemann, A., Echtermeyer, F., von der Mark, H., Miosge, N., Pöschl, E., von der Mark, K., 1997. Absence of integrin alpha-7 causes a novel form of muscular dystrophy. Nat. Genet. 17, 318-323. 
Middleton, C.A., Nongthomba, U., Parry, K., Sweeney, S.T., Sparrow, J.C., Elliott, C.J 2006. Neuromuscular organization and aminergic modulation of contractions in the Drosophila ovary. BMC Biol. 4, 17-21.

Miosge, N., 2001. The ultrastructural composition of basement membranes in vivo. Histol. Histopathol. 16 (4), 1239-1248.

Molnar, J., Ujfaludi, Z., Sheri, F.T., Fong, S.T.F., Bollinger, J.A., Waro, G., Fogelgren, B., Dooley, D.M., Mink, M., Katalin Csiszar, K., 2005. Drosophila lysyl oxidase Dmloxl-1 and Dmloxl-2 are differentially expressed and the active DmLOXL-1 influences gene expression and development. J. Biol. Chem. 280, 22977-22985.

Monson, J.M., Natzle, J., Friedman, J., McCarthy, B.J., 1982. Expression and novel structure of a collagen gene in Drosophila. Proc. Natl. Acad. Sci. U. S. A. 79, 1761-1765.

Nicot, A.-S., Toussaint, A., Tosch, V., Kretz, Ch., Wallgren-Pettersson, C., Iwarsson, E. Kingston, H., Garnier, J.-M., Biancalana, V., Oldfors, A., Mandel, J.-L., Laporte, J., 2007. Mutations in amphiphysin 2 (BIN1) disrupt interaction with dynamin 2 and cause autosomal recessive centronuclear myopathy. Nat. Genet. 39, 1134-1139.

Ortega, N., Werb, Z., 2002. New functional roles for non-collagenous domains of basement membrane collagens. J. Cell Sci. 115, 4201-4214.

Plaisier, E., Gribouval, O., Alamowitch, S., Mougenot, B., Prost, C., Verpont, M.Ch., Marro, B., Desmettre, Th., Cohen, S.Y., Roullet, E., Dracon, M., Fardeau, M., Van Agtmael, T., Kerjaschki, D., Antignac, C., Ronco, P., 2007. COL4A1 mutations and hereditary angiopathy, nephropathy, aneurysms, and muscle cramps. N. Engl. J. Med. 357, 2687-2695.

Pöschl, E., Schlötzer-Schrehardt, U., Brachvogel, B., Saito, K., Ninomiya, Y., Mayer, U. 2004. Collagen IV is essential for basement membrane stability but dispensable for initiation of its assembly during early development. Development 131 $1619-1628$

Razzaq, A., Robinson, I.M., McMahon, H.T., Skepper, J.N., Su, Y., Zelhof, A.C., Jackson, A.P., Gay, N.J. O'Kane, C.J, 2001. Amphiphysin is necessary for organization of the excitation-contraction coupling machinery of muscles, but not for synaptic vesicle endocytosis in Drosophila. Genes Dev. 15 (22), 2967-2979.

Rheault, M.N., Kren, S.M., Thielen, B.K., Mesa, A.H.A., Crosson, J.T., Thomas, W., Sado, Y Kashtan, C.E., Segal, Y., 2004. Mouse model of X-linked Alport syndrome. J. Am. Soc. Nephrol. 15, 1466-1474.

Rodriguez, A.Z., Tang, Zh.M.L., Meller, S., Chen, J., Bellen, H., Kimbrell, D.A., 1996. Identification of immune system and response genes, and novel mutations causing melanotic tumor formation in Drosophila melanogaster. Genetics 143, 929-940.
Sado, Y, Kagawa, M., Naito, I, Ueki, Y Seki, T, Momota, R Oohashi, T, Ninomiya, Y., 1998. Organization and expression of basement membrane collagen IV genes and their roles in human disorders. J. Biochem. 123 (5), 767-776.

Sambrook, J., Fritsch, E.F., Maniatis, T., 1989. Molecular Cloning: a Laboratory Manual, 2nd ed. Cold Spring Harbor Laboratory, Cold Spring Harbor.

Sanes, J.R., 2003. The basement membrane/basal lamina of skeletal muscle. J. Biol. Chem. 278, 12601-12604.

Sempere, L.F. Sokol, N.S., Dubrovsky, E.B., Berger, E.M., Ambros, V., 2003. Temporal regulation of microRNA expression in Drosophila melanogaster mediated by hormonal signals and broad-complex gene activity. Dev. Biol. 259, 9-18.

Suzuki, D.T., 1970. Temperature-sensitive mutations in Drosophila melanogaster. Science 170, 695-706.

Suzuki, D.T., Procunier, D., 1969. Temperature-sensitive mutations in Drosophila melanogaster, III. Dominant lethals and semilethals on chromosome 2. Proc. Natl. Acad. Sci U. S. A. 62, 369-376.

Szidonya, J., Reuter, G., 1988. Cytogenetic analysis of the echinoid (ed), dumpy (dp) and clot (cl) region in Drosophila melanogaster. Genet. Res. Camb. 51, 197-208.

Szidonya, J., Farkas, T., Pali, T., 1990. The fatty acid constitution and ordering state of membranes in dominant temperature-sensitive lethal mutation and wild-type Drosophila melanogaster larvae. Biochem. Genet. 28 (5-6), 233-246.

Tosch, V., Rohde, H.M., Tronchere, H., Zanoteli, E., Monroy, N., Kretz, C., Dondaine, N., Payrastre, B., Mandel, J.-L, Laporte, J., 2006. A novel PtdIns3P and PtdIns(3,5)P2 phosphatase with an inactivating variant in centronuclear myopathy. Hum. Mol. Genet. 15, 3098-3106.

Török, T., Tick, G., Alvarado, M., Kiss, I., 1993. P-lacW insertional mutagenesis on the second chromosome of Drosophila melanogaster: isolation of lethals with different overgrowth phenotypes. Genetics 135, 71-80.

Tryggvason, K., Zhou, J., Hostikka, S.L., Shows, T.B., 1993. Molecular genetics of Alport syndrome. Kidney Int. 43 (1), 38-44.

Vahedi, K., Kubis, N., Boukobza, M., Arnoult, M., Massin, P., Tournier-Lasserve, E., Bousser, M.G., 2007. COL4A1 mutation in a patient with sporadic, recurrent intracerebral hemorrhage. Stroke 38, 1461-1464.

Yasothornsrikul, S., Davis, W.J., Cramer, G., Kimbrell, D.A., Dearolf, C.R., 1997. Viking: identification and characterization of a second type IV collagen in Drosophila. Gene $198,17-25$. 


\section{Article II.}

Drosophila type IV collagen mutation associates with immune system activation and intestinal dysfunction

Márton Kiss ${ }^{\mathrm{a}}$, András A. Kiss ${ }^{\mathrm{a}}$, Monika Radics ${ }^{\mathrm{a}}$, Nikoletta Popovics ${ }^{\mathrm{a}}$, Edit Hermesz ${ }^{\mathrm{b}}$, Katalin Csiszár ${ }^{\mathrm{c}}$ and Mátyás Mink ${ }^{\mathrm{a}}$

Matrix Biology (2016)

https://doi.org/10.1016/j.matbio.2015.09.002

MTMT azonosító: 2962945

IF: $7.4(Q 1)$ 
Matrix Biology

Drosophila type IV collagen mutation associates with immune system activation and intestinal dysfunction

\author{
Márton Kiss ${ }^{\text {a }}$, András A. Kiss ${ }^{a}$, Monika Radics ${ }^{a}$, Nikoletta Popovics ${ }^{a}$, \\ Edit Hermesz $^{\mathbf{b}}$, Katalin Csiszár ${ }^{\mathrm{c}}$ and Mátyás Mink ${ }^{\mathrm{a}}$ \\ a - Department of Genetics, University of Szeged, Középfasor 52, H-6726 Szeged, Hungary \\ b - Department of Biochemistry and Molecular Biology, University of Szeged, Középfasor 52, H-6726 Szeged, Hungary \\ c - John A. Burns School of Medicine, University of Hawaii, 1960 East West Road, Honolulu, HI 96822, USA
}

Correspondence to Mátyás Mink: mink@bio.u-szeged.hu.

http://dx.doi.org/10.1016/j.matbio.2015.09.002

Edited by R. Iozzo

\begin{abstract}
The basal lamina (BM) contains numerous components with a predominance of type IV collagens. Clinical manifestations associated with mutations of the human COL4A1 gene include perinatal cerebral hemorrhage and porencephaly, hereditary angiopathy, nephropathy, aneurysms and muscle cramps (HANAC), ocular dysgenesis, myopathy, Walker-Warburg syndrome and systemic tissue degeneration. In Drosophila, the phenotype associated with dominant temperature sensitive mutations of col4a1 include severe myopathy resulting from massive degradation of striated muscle fibers, and in the gut, degeneration of circular visceral muscle cells and epithelial cells following detachment from the BM. In order to determine the consequences of altered BM functions due to aberrant COL4A1 protein, we have carried out a series of tests using Drosophila DTS-L3 mutants from our allelic series of col4a1 mutations with confirmed degeneration of various cell types and lowest survival rate among the col4a1 mutant lines at restrictive temperature. Results demonstrated epithelial cell degeneration in the gut, shortened gut, enlarged midgut with multiple diverticulae, intestinal dysfunction and shortened life span. Midgut immunohistochemistry analyses confirmed altered expression and distribution of BM components integrin PSI and PSII alpha subunits, laminin gamma 1, and COL4A1 both in larvae and adults. Global gene expression analysis revealed activation of the effector AMP genes of the primary innate immune system including Metchnikowin, Diptericin, Diptericin B, and edin that preceded morphological changes. Attacin::GFP midgut expression pattern further supported these changes. An increase in ROS production and changes in gut bacterial flora were also noted and may have further enhanced an immune response. The phenotypic features of Drosophila col4a1 mutants confirmed an essential role for type IV collagen in maintaining epithelial integrity, gut morphology and intestinal function and suggest that aberrant structure and function of the COL4A1 protein may also be a significant factor in modulating immunity.
\end{abstract}

(C) 2015 Published by Elsevier B.V.

\section{Introduction}

The polarization and function of the epithelia including mucosal epithelia is determined and supported by a specialized form of the extracellular matrix (ECM), the basal lamina or basement membrane (BM). In various tissues and body compartments, the $\mathrm{BM}$ also fulfills diverse functions including separation, absorption, sensation and secretion [1]. The BM is made of numerous components with a predominance of type IV collagens. Mammals, including humans, harbor three pairs of type IV collagen genes (COL4A1-6). The inherited disorder of renal, ocular and cochlear basement membranes associates with mutations of the $\mathrm{X}$-linked COL4A5gene in the majority of patients with Alport syndrome, whereas mutations in the autosomal COL4A3 and COL4A4 genes are responsible for the symptoms of Alport syndrome in 
about $20 \%$ of patients [2,3]. In the mammalian BM, the dominant type IV collagen units are trimers with (COL4A1) ${ }_{2}$ COL4A2 composition.

Clinical manifestations associated with mutations of COL4A1 include perinatal cerebral hemorrhage and porencephaly [4], hereditary angiopathy, nephropathy, aneurysms, and muscle cramps (HANAC) [5], ocular dysgenesis, myopathy and Walker-Warburg syndrome [6]. The latest reports also demonstrated systemic tissue degeneration

A

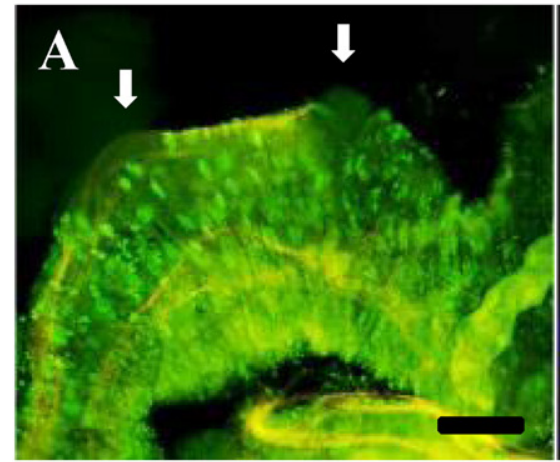

\section{B}
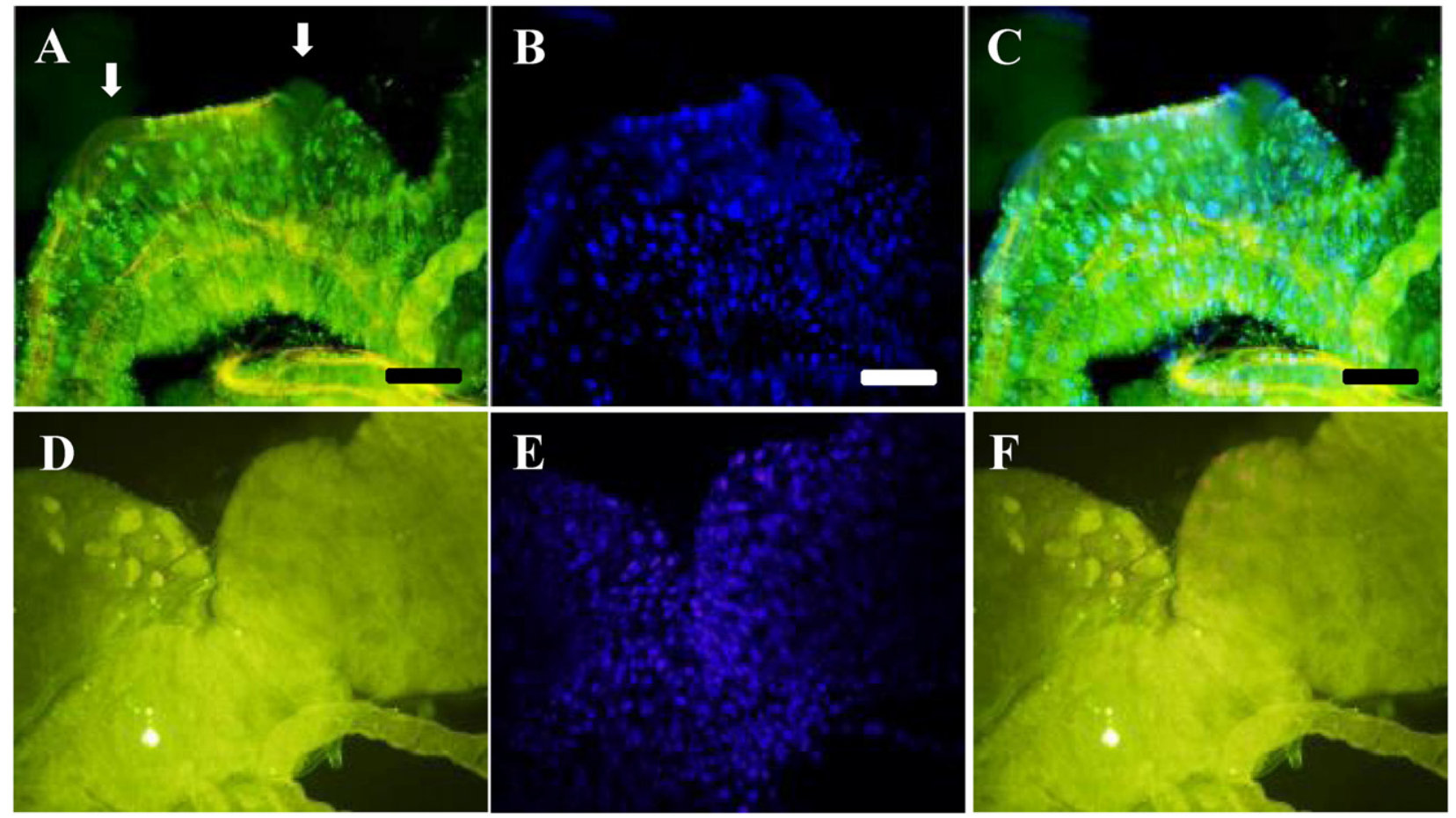

B
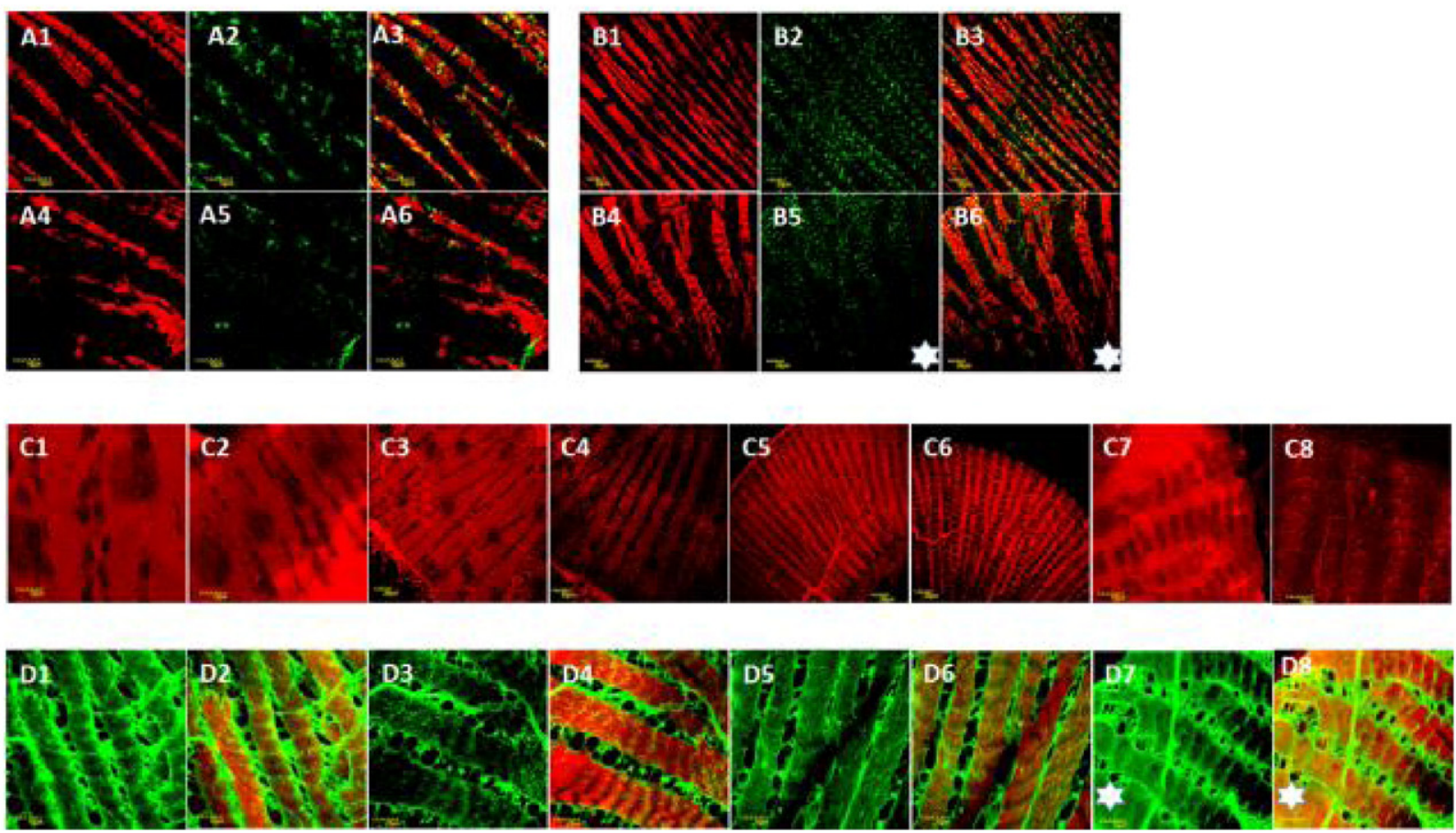

Fig. 1 (legend on next page). 
and pleiotropy associated with COL4A1 mutations and confirmed that the phenotypes of COL4A2 mutations are phenocopies of COL4A1 gene mutations [7].

We have recently reported an allelic series of conditional, dominant, temperature-sensitive (DTS) mutations within the type IV collagen gene (col4a1) in Drosophila. The col4a1 mutant heterozygotes are viable and fertile at permissive temperature $\left(20^{\circ} \mathrm{C}\right)$, but perish at restrictive temperature $\left(29^{\circ} \mathrm{C}\right)$. The phenotype associated with mutations of col4a1 include severe myopathy resulting from massive degradation of striated muscle fibers [8], degeneration of the circular visceral muscle cells and the epithelial cells of the gut that occur following detachment from the BM [9]. While there are signs of repair, the capacity of the scavenger system, and/ or the kinetics of cell renewal and regeneration fail to keep up with the ongoing cell degeneration in these mutants $[8,9]$.

The mucosal epithelia of the gastrointestinal tract are constantly challenged by the microbiome resulting in various types of interactions including commensalism, symbiosis and pathogenicity [10]. Drosophila is one of few animal models in which host-microbe interactions in the gut have been studied [11]. The gut-associated bacterial community in Drosophila is scarce, harboring $5-10$ bacterial phylotypes [12] and among these five dominant commensal species: Lactobacillus plantarum, Lactobacillus brevis, Acetobacter pomorum, Gluconobacter morbifer and Commensalibacter intestini $[13,14]$. Drosophila antimicrobial defense is facilitated by the phagocytosis of pathogenic microorganisms and by the synthesis of antimicrobial peptides (AMPs). Epithelial defense also relies on the generation of reactive oxygen species (ROS) [15-17].

Activation of the immune system by overexpression of AMPs, including Diptericin, Cecropin, Drosomycin, Defensin, Attacin and Metchnikowin, as demonstrated in caudal hypomorphic RNAi mutants, results in restructuring of the commensal bacterial population with the dominance of the pathogenic Gluconobacter morbifer accompanied by degradation of gut epithelial cells and high mortality of the host $[13,14]$ suggesting a role for deregulated expression of AMPs in epithelial cell degeneration and gut pathogenesis. Overexpression of AMPs was similarly linked in aging flies to dysfunction of the intestinal barrier that normally permits the absorption of nutrients and solutes and hampers host contact with harmful entities $[18,19]$. Compromised intestinal barrier function was also noted in big bang $(b b g)$ null Drosophila mutants that in the absence of the BBG protein that is localized in gut epithelial septate junctions, demonstrated reduced lifespan and chronic inflammation of the anterior midgut epithelium [20]. While some of the col4a1 mutation-associated phenotypic features were consistent with chronic inflammation, inflammatory and immune-activation mechanisms have not been explored either in Drosophila col4a1 or in association with mouse or human COL4A1 mutations $[5,6,7,13,14]$.

In order to determine if mutated col4a1 and the resulting aberrant BM induced cell degeneration, compromised gut mucosal epithelial functions and activated the immune system, we have carried out a series of tests in mutant animals including evaluation of larval development, gut morphology, integrity of epithelial and visceral muscle cells of the gut, degree of cell death, immunohistochemistry of BM proteins in the midgut, global gene expression changes, expression values of the activated AMP effector genes of the primary innate immune system, changes in AMP protein Attacin::GFP levels in the midgut, and ROS production. We have also analyzed changes in the composition of the gut bacterial flora and the tolerance of bacterial load as indicators of modified immune functions, and recorded changes in life-span. From our allelic series of col4a1 mutations, we have selected for these analyses the DTS-L3 mutant based on the confirmed degeneration of various cell types and lowest survival rate among the col4a1 mutant lines at restrictive temperature of $29^{\circ} \mathrm{C}[8,9]$.

\section{Results}

\section{Aberrant gut morphology, cell degeneration and altered expression of BM components in the midgut of col4a1 mutants}

Massive cell degeneration that we have described earlier $[8,9]$ was confirmed at restrictive temperature $\left(29^{\circ} \mathrm{C}\right)$ in the midgut of col4a1 adult mutant flies carrying the dominant temperature-sensitive DTS-L3 allele. Additionally, the following phenotype changes were noted: L3-stage DTS-L3 mutant larvae were shorter in length $(4.9+/-0.3 \mathrm{~mm})$ at $29^{\circ} \mathrm{C}$ compared to values $(5.5+/-0.4 \mathrm{~mm})(\mathrm{p}=0.032)$ at $20^{\circ} \mathrm{C}$ and to wild-type larvae at either temperatures. Gut alterations

Fig. 1. A. Fluorescence micrographs demonstrating TUNEL positivity in larval midgut. A: TUNEL-positive midgut of a DTS-L3 L3-larva incubated at $29^{\circ} \mathrm{C}$; arrows point to diverticulae and to protruding peritrophic matrix. B: DAPI, C. Merge. D, E, F: Midgut of a DTS-L3 L3-larva incubated at $20^{\circ} \mathrm{C}$ appears TUNEL-negative. Scale bars: $50 \mu \mathrm{m}$. B. Actin (red) and integrin (green) staining of the L3 larval midgut visceral muscle cells. A1: Actin, A2: Integrin, A3: Merge, wild-type; A4-6, mutant, same order, $20^{\circ} \mathrm{C}$. B1-3, wild type, B4-6 mutant, $29^{\circ} \mathrm{C}$. Asterisks refer to areas of lower integrin expression. C1, C2: Laminin expression (red) in the midgut of $\mathrm{L} 3$ control/mutant larvae, $20^{\circ} \mathrm{C}, \mathrm{C} 3, \mathrm{C} 4$, at $29^{\circ} \mathrm{C}$. C5, C6: Laminin expression in the midgut of control/mutant adults, $20^{\circ} \mathrm{C}, \mathrm{C} 7, \mathrm{C} 8,29^{\circ} \mathrm{C}$. Note decreased laminin expression in $\mathrm{C} 4$ and $\mathrm{C} 8$. 
included shorter gut (1.5+/-0.4 arbitrary units) compared to those of wild-type larvae $(1.9+/-0.3$ arbitrary units) irrespective of the incubation temperature. In the transparent larvae, pathological gut morphology included enlarged midgut with diverticulae at several sites (Fig. 1A), whereas the midgut of the control larvae had a flat surface at $29^{\circ} \mathrm{C}$ (Fig. 1D).
Fluorescent light microscopic inspection revealed additional protruding sites/diverticulae along the midgut. The nuclei within areas of the diverticulae proved TUNEL-positive involving nearly all nuclei (Fig. 1A-C). Areas of the diverticulae, furthermore, lacked cellular organization and presented a protruding peritrophic matrix (Fig. $1 \mathrm{~A}-\mathrm{C}$ ) while the midgut of the
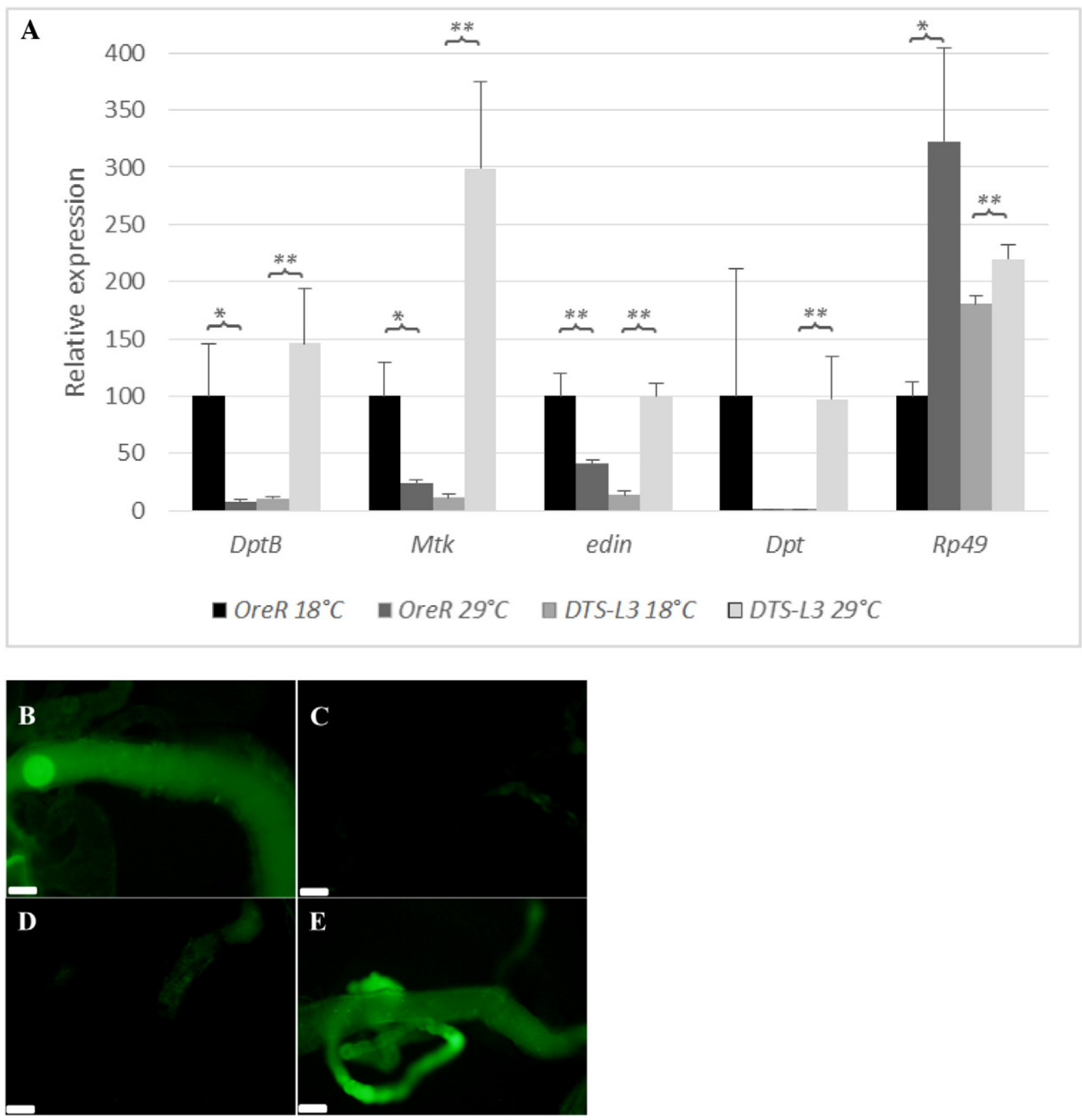

Fig. 2. A. QRT-PCR validation of the expressional data of selected AMP genes. Metchnikowin (Mtk), Diptericin (Dpt), Diptericin $B(D p t B)$ and edin in DTS- $L 3$ mutants, following a three-day shift to restrictive temperature ${ }^{\star} p<0.05 ;{ }^{* *} p<0.01$. Expression values of the ribosomal protein 49 gene (Rp49) served as an internal control. ATT::GFP expressions in control (Fig. 2B) and mutant (Fig. 2C) flies at permissive temperature and at $29{ }^{\circ} \mathrm{C}$ (Fig. 2B, D, respectively). Scale bars: $100 \mu \mathrm{m}$. Note the opposite expressional levels in the wild-type and mutant animals. 
mutant larvae reared at $20^{\circ} \mathrm{C}$ did not show these features (Fig. 1D-F).

The expression and distribution of BM components integrin PSI and PSII alpha subunits, laminin gamma 1 and collagen COL4A1 were analyzed in the midgut using immonohistochemistry (Fig. 1B). Panels A1-3 present the visceral muscle cells of the midgut in control L3 larvae, A4-6 correspond to mutant midgut stained for integrins (green) and actin (red) at $20^{\circ} \mathrm{C}$. In control larvae, punctate integrin staining localized to the Z-disks while in mutants slightly less integrin expression was detected at this location. This tendency was more apparent in larvae reared at $29^{\circ} \mathrm{C}$ where large areas in the midgut of the mutant lacked integrin expression (asterisks, Fig. 1 B1-3 vs. B4-6). The same integrin expression pattern was observed in the midgut of adult mutant animals (Supplementary Fig. S1). Laminin gamma 1 was similarly reduced in the midgut of mutant L3 larvae (Fig. 1B, C3 vs. C4, red) and in the midgut of adult animals (Fig. 1B, C7 vs. C8, red) at $29^{\circ} \mathrm{C}$ and appeared as a diffuse staining in mutants (C4, C9). COL4A1 staining accumulated in the sarcoplasm in the midgut of the mutant at $20^{\circ} \mathrm{C}$ (Fig. 2B, D3, D4, green) and indicated a diffuse, irregular staining pattern at $29^{\circ} \mathrm{C}$ (Fig. 2B, D7, D8, green, asterisks). These results corroborated our earlier data and confirmed a systemic, multi-organ phenotype in col4a1 mutants at restrictive temperature $[8,9]$.

\section{Overexpression of AMP genes of the primary innate immune system and elevated oxidant production in col4a1 DTS-L3 mutants}

DNA-array experiments were carried out to detect col4a1 mutation-associated gene expressional alterations in DTS-L3 mutant animals compared to wild-type control flies at both permissive and restrictive temperatures [8]. Most of the upregulated genes in the mutants at restrictive temperature were related to immunological functions including host defense response, antibacterial humoral response, and innate immune response (Supplementary Table 1). The expression of certain peritrophic matrix constituents (CG10154 and CG10725), as well as genes for digestive tract enzymes (CG17234 and CG31091) and a transmembrane transport protein (CG9825) were also elevated in the mutants. In wild type Oregon files, the expression patterns for all of these genes followed an opposite tendency and were downregulated at elevated temperature. We have also found a gene, CG13043 that was significantly downregulated in the mutant but was upregulated in wild type flies. The CG13043 gene encodes a Retinin domain protein (pfam04527), considered to be a cuticle protein with yet unknown function that is expressed in the trachea and the testis.

Genes His3 and Hsp22 were upregulated and one of the peritropic matrix protein genes, obst- $B$, was downregulated in both mutant and control samples likely as part of an orchestrated response to elevated temperature. BobA and Dup99B were also found underexpressed in both mutants and controls. BobA is responsible for Notch signaling, while Dup99B plays a role in oviposition. Both genes seemed to be temperature-regulated regardless of mutational status.

In col4a1 mutants, AMP genes of the primary innate immune system Metchnikowin, Diptericin, Diptericin B, Attacin A, Attacin C and edin (elevated during infection) showed upregulation in the mutants whereas downregulation in controls at restrictive condition at statistically significant $(p<0.01)$ levels. Results of the DNA-array experiments are summarized in Supplementary Table 1 and gene expression data were deposited in the GEO-Database under accession number GSE52579.

DNA-array expression values for the AMP genes were validated by QRT-PCR. The data corroborated values of the DNA-array results and confirmed low levels of expression at $20^{\circ} \mathrm{C}$ and significantly increased expression of Metchnikowin (Mtk), Diptericin $(D p t)$, Diptericin B (DptB) and edin in mutants following a three-day shift to restrictive temperature, while in control animals, these genes showed significant downregulation at $29^{\circ} \mathrm{C}$ (Fig. 2A).

Expression changes at protein level were established utilizing the Drosophila transgenic line carrying the Attacin::Green Fluorescent Protein (GFP) fusion under the control of the Att promoter [39]. Att mRNA expression was downregulated $\log 2=-2,29$-fold in wild-type control animals, whereas upregulated in mutants log2 $=3,19$-fold following a shift to $29^{\circ} \mathrm{C}$ (Supplementary Table 1). Protein levels followed the pattern of transcriptional alterations. In controls, reduced amount of ATT::GFP (Fig. 2B, D), whereas in mutant animals increased amount of fusion protein was detected at $29^{\circ} \mathrm{C}$ (Fig. 2C, E).

In order to evaluate whether an increase in oxidant production also occurred in mutants, we have measured the concentrations of two oxidants peroxynitrite and hydrogen peroxide at both $20^{\circ} \mathrm{C}$ and $29^{\circ} \mathrm{C}$. In mutants, peroxynitrite production was detected at higher concentrations compared to controls regardless of the temperature (Fig. 3). Hydrogen peroxide concentrations increased statistically significantly $(p<0.05)$ in the mutant and were slightly elevated in control animals at $29^{\circ} \mathrm{C}$ (Fig. 3).

\section{Bacterial species and counts are reduced in the col4a1 mutant gut niche}

In order to determine if altered expression of the primary/innate immune system had functional consequences in mutant animals, we have analyzed the gut microbial flora and compared to normal controls. Amplification and sequencing of the $16 \mathrm{~S}$ ribosomal RNA genes of the commensal bacteria from both 


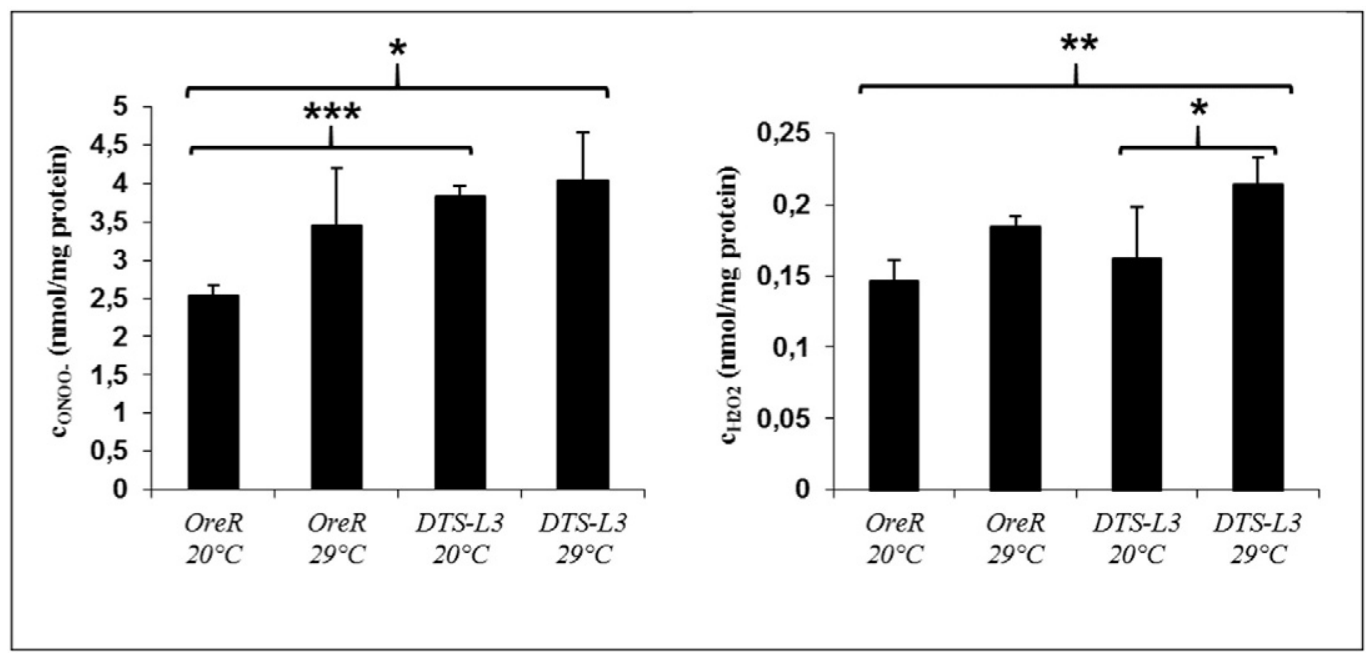

Fig. 3. Measurements of $\mathrm{ONOO}^{-}$and $\mathrm{H}_{2} \mathrm{O}_{2}$ concentrations in $\mathrm{nmol} / \mathrm{mg}$ protein units in control and mutant animals at both permissive and restrictive temperatures. All values are presented as means \pm S.D. ${ }^{*} p<0.05,{ }^{* *} p<0.005,{ }^{* *} p<0.001$.

mutants and controls kept at elevated temperature for 3 days was carried out. BLAST searches of the nucleotide sequences of the amplified fragments identified two common bacterial phylotypes, Acetobacter cerevisiae and Lactobacillus plantarum in both lines. As the composition of the intestinal microbiome changes dynamically and is dependent on environmental factors, we also cultivated both mutant and wild-type flies on starch-based medium. Under these conditions, beyond Acetobacter and Lactobacillus a third cultivable member of the gut-associated microbial community appeared in form of red colonies that, upon morphologic evaluation and ITS sequence analysis, proved to be yeast Rhodothorula species. Following a shift to sucrose-based food, Rhodothorula disappeared from the gut niche. The titers of the microbial community were also determined in the gut of mutant and wild-type flies. The number of colony-forming units
(CFU) was $\sim 10^{5}$ per gut both in mutants and controls at $20^{\circ} \mathrm{C}$. At $29^{\circ} \mathrm{C}$, statistically significant reduction ( $p=0.015)$ in CFU values was recorded in wild-type flies and while not statistically significant, a decrease of CFU was also noted in mutants. The elevated oxidant concentration (Fig. 3) and increased rate of oxidative reactions at $29{ }^{\circ} \mathrm{C}$ were consistent with the observed decrease in CFU values of the intestinal microbiome (Fig. 4).

Importantly, in mutants, the Acetobacter/Lactobacillus ratios revealed a statistically significant shift toward Acetobacter, a feature that was already present at permissive temperature (Fig. 4).

\section{Increasing the bacterial dose is lethal at $29^{\circ} \mathrm{C}$}

The lifespan of the wild-type control and mutant flies is 105 days that does not differ significantly at permissive temperature. Rearing both lines at $29^{\circ} \mathrm{C}$

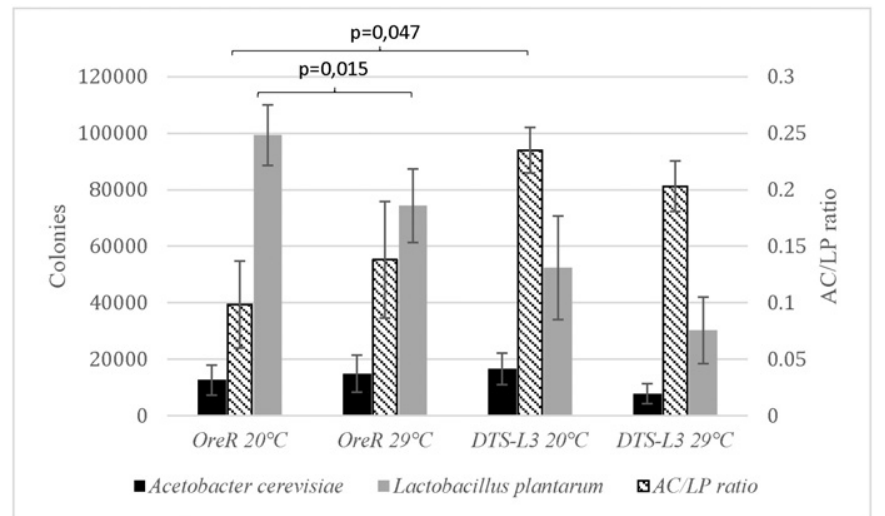

Fig. 4. CFU values of Acetobacter cerevisiae and Lactobacillus plantarum in DTS-L3 and wild-type flies incubated at $20^{\circ} \mathrm{C}$ and $29^{\circ} \mathrm{C}$. Left y axis: colony count/gut; right y axis: A. cerevisiae/L. plantarum (AC/LP) ratios. 


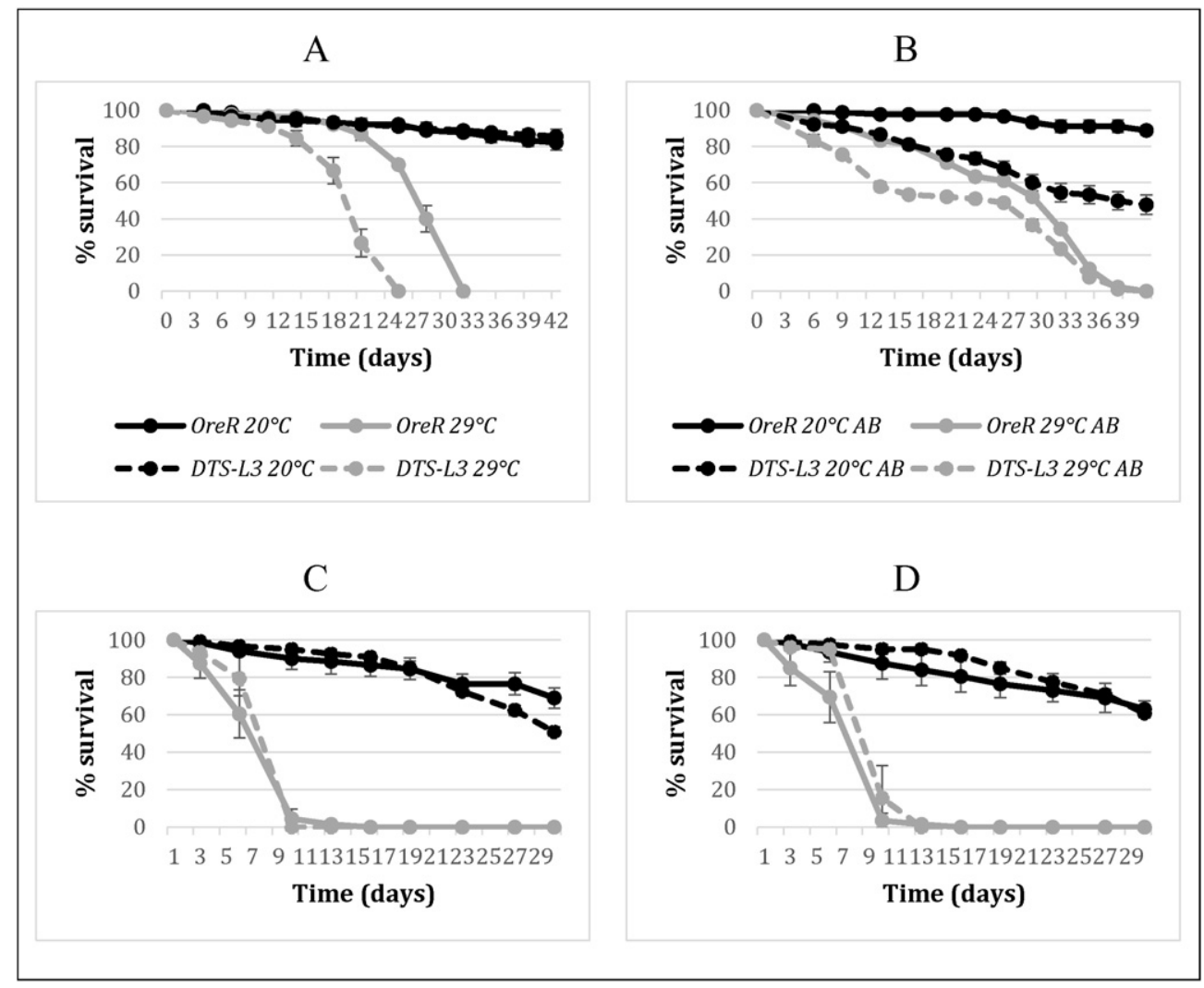

Fig. 5. A: Survival of mutant and control flies, expressed as percentage of escapers, at both permissive and restrictive temperatures on normal food. B: Effect of antibiotic treatment on survival, C: Survival on Acetobacter cerevisiae, D: Survival on Lactobacillus plantarum. Legends to Figures $\mathrm{C}$ and $\mathrm{D}$ are the same as in Figure $\mathrm{A}$.

reduced longevity of the wild-type flies to 32 days, whereas to 25 days of mutants (Fig. 5A).

To test the hypothesis that increased levels of the normal gut-associated bacteria may affect the lifespan, isolated microbiotes were orally fed to both mutant and wild-type flies. The survival rates of the orally infected flies revealed that neither Lactobacillus plantarum nor Acetobacter cerevisiae influenced the life span of either mutant or wild-type flies at $20^{\circ} \mathrm{C}$ (Fig. 4C, D), and the CFU values in the gut remained in the range observed in the uninfected population $\sim 10^{5} \mathrm{CFU} /$ gut (not shown). In contrast, at $29^{\circ} \mathrm{C}$, both mutant and wild-type flies succumbed following ten days of oral infection (Fig. 4C, D) irrespective the bacteria used with dramatically increased intestinal CFU values, exceeding 1$4 \times 10^{6} \mathrm{CFU} /$ gut (not shown). These results showed that a young population of both mutant and wild-type flies maintain relatively constant intestinal CFU values at $29^{\circ} \mathrm{C}$ and suggested that the composition and titer of the intestinal microbial community does not contribute remarkably to the pathology of the young DTS-L3 mutants, and that their reduced lifespan ( 25 versus 32 days) may be due to other factors.
In aging flies, in contrast, increased bacterial loads have been demonstrated by several laboratories and were suggested to contribute to the death of the animals [19,21-23]. Premature aging and death of the col4a1 mutant flies might be caused by earlier onset of increased bacterial load, therefore we anticipated extended life span of the mutants by rearing them on antibiotics at restrictive temperature. Indeed, lifelong clearing of bacteria increased the life expectancy of both mutant and control lines up to 38 days at $29^{\circ} \mathrm{C}$ (Fig. 5B). These results were consistent with previous experimental evidence $[19,21-23]$ and confirmed that elevated bacterial load is malign in aging flies but did not explain why col4a1 mutant flies still succumbed significantly earlier on antibiotics-free medium.

\section{Premature loss of intestinal integrity in col4a1 mutants}

Detachment and TUNEL-positive staining of the gut epithelial and visceral muscle cells in the DTS-L3 mutants at restrictive condition (Fig. 1), [9] indicated loss of intestinal integrity. Therefore, we tested barrier function of the alimentary tract by monitoring 

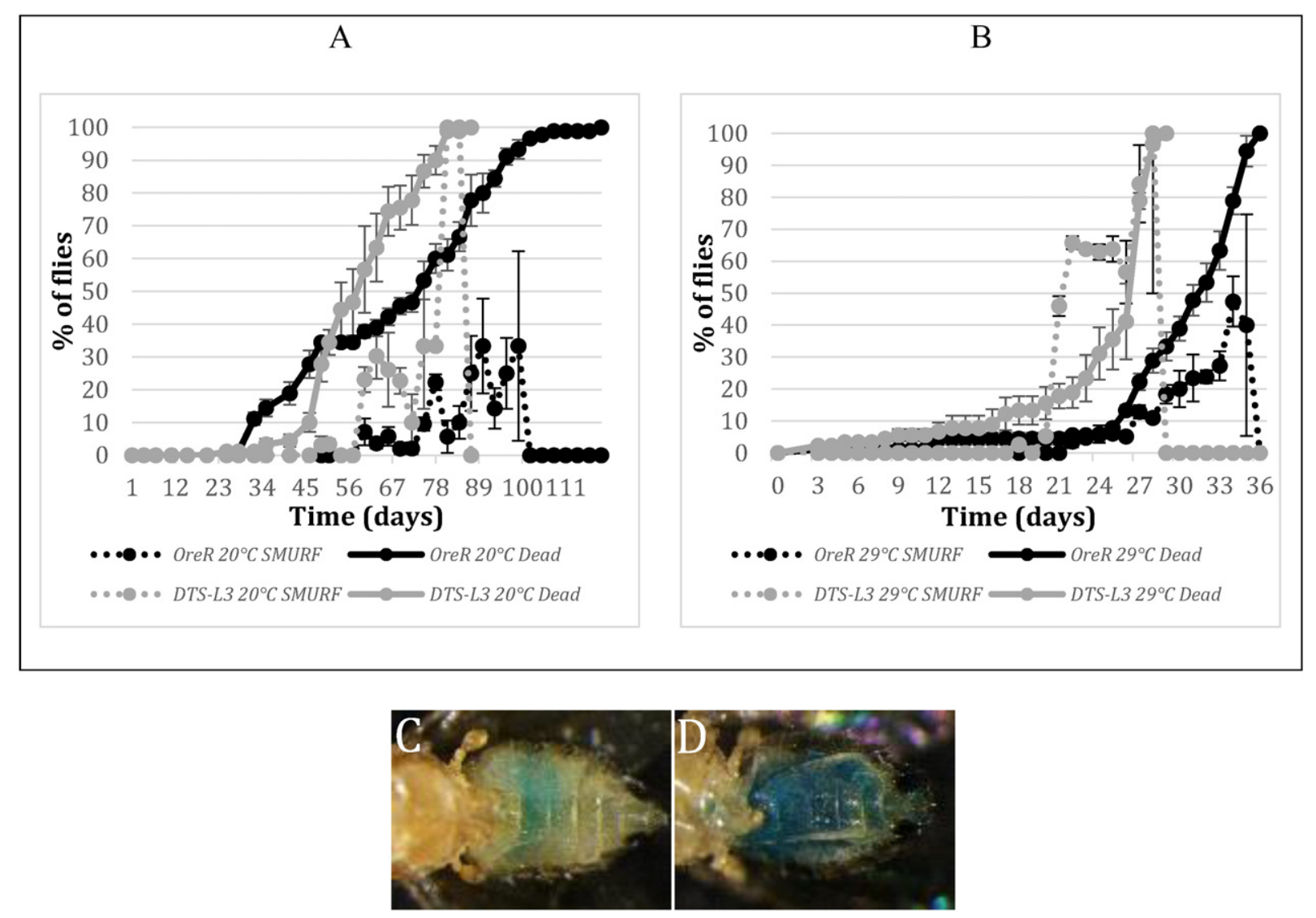

Fig. 6. A: Onset of SMURF phenotype and survival of DTS-L3 and wild-type flies incubated at $20{ }^{\circ} \mathrm{C}$, and $\mathrm{B}$ : at $29{ }^{\circ} \mathrm{C}$. C: Staining of the intact intestine in young mutant. D: SMURF phenotype marked by staining of the hemolymph in an aging mutant fly with abrupt onset of degradation of intestinal integrity and death of the animals at 20-22nd day of incubation.

the onset of the SMURF phenotype, i.e. penetrance of non-absorbable blue food dye into the hemolymph in both mutants and controls. At $20^{\circ} \mathrm{C}$, intestinal dysfunction did not occur until 57 days of incubation in either mutants or controls with no significant differences between the two lines (Fig. 6A). Incubation at $29{ }^{\circ} \mathrm{C}$, however, resulted in an abrupt onset of the SMURF phenotype in DTS-L3 mutants (Fig. 6B) between 20 and 22 days followed by immediate death of the animals (Fig. 6A). Intestinal dysfunction in wild-type flies did not occur within this time-frame (Fig. 5C, D). These results suggested that premature aging of the DTS-L3 mutants was due to dysfunction of the intestinal barrier that also explained their short lifespan.

\section{Discussion}

The components and three-dimensional structure of the ECM provide a microenvironment that regulates basic cellular functions. Aberrant expression and structure of the ECM during tissue remodeling or pathological processes activate the immune system and influence cell survival. Degraded fragments of the
BM also modulate the activities of both resident cells and infiltrating immune cells [24]. Collagen type IV fragments resulting from BM degradation by metalloproteinases were implicated in the synthesis of antimicrobial peptides (AMPs) and stimulation of innate immune responses during insect metamorphosis [25]. Endogenously derived danger/alarm signals, including collagen type IV fragments, also stimulated immune cells in lepidoptera [26]. While modulated genetic elements for components of the BM (laminin, nidogen/entactin, perlecan, integrins and crosslinking lysyl oxidases) are available in Drosophila [27-29], the specific role of aberrant BM and mutated collagen type IV in modulating immune functions has not been explored in this model.

We have demonstrated earlier that in temperaturesensitive col4a1 Drosophila mutants altered BM structure and function cause degradation of skeletal and visceral muscle cells resulting in severe myopathy $[8,9]$. Results of the current study confirmed that mutated col4a1 also causes degeneration of epithelial cells of the gut at restrictive temperature. Following continuous incubation of mutants at $29^{\circ} \mathrm{C}$ from egg deposition, morphological changes manifested in 
shorter larvae and the development of severe gut pathology including shorter gut, enlarged midgut, and multiple diverticulae collectively leading to intestinal dysfunction and shortened life span.

In adult animals, three days of temperature challenge and the resulting massive cell degradation as part of an early response, was sufficient to activate the immune system manifested in the overexpression of AMP genes Metchnikowin, Diptericin, Diptericin B, and edin. In contrast to mutants, wild-type control flies downregulated AMP synthesis at $29^{\circ} \mathrm{C}$ in agreement with the reported downregulation of Metchnikowin in flies kept at $29^{\circ} \mathrm{C}$ [30].

Multiple lines of evidence suggest a direct relationship between AMP overexpression, apoptosis of gut epithelial cells, and mortality reported for both caudal Drosophila mutants and aging animals $[13,18,19]$. In col4a1 mutants, temperature-induced initiation of epithelial cell detachment from the BM, activation of the immune system, and subsequent intestinal dysfunction appeared to be the primary causes of their abrupt and premature death. Elevated oxidant concentrations may have additionally promoted epithelial damage and intestinal dysfunction.

A shift in gut bacterial species and decrease of the CFU values were temperature-dependent responses irrespective of col4a1 mutation. Immune deficiency pathway-mutants have a contrasting higher gut CFU values, indicating that the basal level of antimicrobial peptides effectively regulate bacterial density [31]. Reduced bacterial count observed in controls with reduced AMPs and in DTS-L3 mutants with overexpressed AMPs suggested that factors other than AMPs contributed to the maintenance of gut homeostasis at elevated temperature.

Already present at permissive temperature, in mutants the Acetobacter/Lactobacillus ratios revealed a statistically significant shift toward Acetobacter. The acetic acid produced by Acetobacter effectively modulates insulin/insulin-like growth factor signaling in Drosophila that regulates developmental rate, body size, energy metabolism, and intestinal stem cell activity [32]. Thus the predominance of Acetobacter in the gut flora of col4a1 mutants may modify additional regulatory mechanisms that contribute to the phenotype.

In DTS-L3 col4a1 mutants, visceral muscle cell degeneration [9] has at least two severe consequences. First, during regeneration of the gut epithelium by the intestinal stem cells, visceral muscle cells that surround the epithelium produce epidermal growth factor Vein [31] a process that may be severely compromised in mutants. Second, compromised visceral muscle cells may cause motility-associated disorders, including life-threatening chronic intestinal pseudo-obstruction (CIPO) [33]. Therefore, Vein deficiency and CIPO may also be contributing factors to premature aging and death of mutants.
Considering the present data a question arises: Why human patients and mice with COL4A1 mutations do not develop chronic inflammation and prominent activation of the immune system? The mammalian genetic alteration differs from the col4a1 Drosophila mutation in several aspects. The homologous Drosophila mutations do not affect viability, fertility or cause detectable BM-associated cell degeneration at permissive temperature. Strong systemic phenotype occurs only following temperature stress. Stress-induced phenotype was similarly observed in Col4a ${ }^{-1-}$ mouse embryos. Col4a1 or Col4a2 null homozygotes were lethal at a developmental phase when the embryonic heart started to beat and put the blood vessels under pressure [34].

Our results with a Drosophila col4a1 conditional dominant mutant line support the suitability of this model to study mechanistic elements of the human COL4-associated condition, mutant phenotypic features confirm an essential role for type IV collagen in maintaining epithelial integrity, gut morphology, and intestinal function, and suggest that aberrant structure and function of the COL4A1 protein may be a significant factor in modulating immunity.

\section{Experimental procedures}

\section{Drosophila maintenance, RNA isolation, labeling, and DNA-array experiments}

Wild-type Oregon flies and col4a1 mutant stock with the DTS-L3 allele were maintained at $20^{\circ} \mathrm{C}$ or $29^{\circ} \mathrm{C}$ on yeast-cornmeal- sucrose-agar medium supplemented with propionic acid and hydroxymethylbenzoate to avoid fungal infections. In the starch-based food, sucrose was replaced by wheat flour. To test the effect of antibiotics we used a combination of ampicillin and tetracycline, dissolved in $50 \%$ ethanol, mixed into the medium to a final concentration of $500 \mu \mathrm{g} / \mathrm{ml}$ and $50 \mu \mathrm{g} / \mathrm{ml}$, respectively. The vials were prepared a day before use and were changed in every 6 days. Intestinal barrier dysfunction was monitored by mixing 30 -fold diluted stock solution of the blue food dye Patent Blue V/E131 in the liquid medium and recording the onset of the diffusion of the dye from the gut into the surrounding tissues (SMURF phenotype).

The col4a1 mutant stock was kept heterozygous over the CyRoi balancer chromosome. Prior to RNA isolation the dominant temperature-sensitive DTS-L3 mutant and wild-type Oregon flies were incubated at $18{ }^{\circ} \mathrm{C}$ and $29^{\circ} \mathrm{C}$ for three days. 24 flies were homogenized following $\mathrm{CO}_{2}$-anesthesy in $500 \mu \mathrm{l}$ of TRIzol@ Reagent (Invitrogen) according to the manufacturer's instructions. To remove residual genomic DNA, RNA samples (5 $\mu \mathrm{g}$ aliquots) were incubated for 30 min at $37^{\circ} \mathrm{C}$ with 1 unit of DNase (Ambion DNA-free), inactivated at room temperature for two minutes by adding the inactivation reagent. RNeasy 
columns (Qiagen) were applied for further purification. $260 / 280$ and $260 / 230$ ratios in all samples were above 2. Agilent microarray analysis was performed using the commercially available $4 \times 44$ k Drosophila platform (Agilent ID: 018,972). $5 \mu \mathrm{g}$ of total RNA was reverse transcribed and used for each condition according to the Agilent Two-color Quick Amp Kit protocol. A technical duplicate was made by dye-swap resulting in eight samples in the following combinations:

\begin{tabular}{llll}
\hline Array 1_1 & \multicolumn{1}{c}{ Array 1_2 } & \multicolumn{1}{c}{ Array 1_3 } & \multicolumn{1}{c}{ Array 1_4 } \\
\hline Ore $20{ }^{\circ} \mathrm{C} /$ Cy5 & Ore $29{ }^{\circ} \mathrm{C} / \mathrm{Cy} 5$ & Ore $20{ }^{\circ} \mathrm{C} / \mathrm{Cy} 3$ & Ore $29{ }^{\circ} \mathrm{C} / \mathrm{Cy} 3$ \\
DTS-L3 & DTS-L3 & DTS-L3 & DTS-L3 \\
$20^{\circ} \mathrm{C} / \mathrm{Cy} 3$ & $29^{\circ} \mathrm{C} / \mathrm{Cy} 3$ & $20^{\circ} \mathrm{C} / \mathrm{Cy} 5$ & $29^{\circ} \mathrm{C} / \mathrm{Cy} 5$ \\
\hline
\end{tabular}

Labeled samples were hybridized in $2 \times$ GEx Hybridization buffer HI-RPM to the array at $65^{\circ} \mathrm{C}, 10$ RPM, for $17 \mathrm{~h}$. The slides were washed sequentially with GE Wash Buffer 1 and 2 and scanned by Agilent G2505B scanner. Microarray data were processed from raw data image files and were quantified with Feature Extraction Software 8.5 (Agilent Technologies). The raw processed signal intensities were normalized to total intensity for comparison between subarrays achieved by Cy5 and Cy3 dyes. Data were filtered by four features: "green is positive and significant", "red is positive and significant", "green is well above background" and "red is well above background". Log2 transformation of normalized values, two sample t-test with equal variances, filtering for $p<0,01$ and averaging of repeated log2 values were calculated. Processed data were subsequently imported and evaluated by the GeneSpring GX and Microsoft Excel. AMIGO gene ontology database was used to find corresponding GO terms; GO annotations were translated by www.sigenae.org.

Validating of the microarray data was achieved using the same templates by QRT-PCR. RevertAid reverse transcriptase (Fermentas) with Random primers (Invitrogen) that were applied for cDNA synthesis and RNaseOut (Invitrogen) was added to avoid RNA degradation. KAPA Sybr Fast Universal qPCR mastermix was used and the reactions were run on an Applied Biosystems 7500 Real-Time PCR System. Expression values in samples of wild-type control flies incubated at $20^{\circ} \mathrm{C}$ served as references. Ct values were corrected with reaction efficiencies and cDNA quantity. Student's t-test was used to calculate statistical significance.

\section{Genotyping of gut bacteria}

Mutant and wild-type flies were incubated for three days at $20^{\circ} \mathrm{C}$ or $29^{\circ} \mathrm{C}$, surfaces were sterilized in $70 \% \mathrm{EtOH}$, guts were dissected from a group of five anesthetized flies and homogenized in $100 \mu \mathrm{l}$ sterile SB broth. $80 \mu \mathrm{l} 100$-fold dilutions of the suspensions were plated on mannitol-agar $(2.5 \%$ n-mannitol,
$0.5 \%$ yeast extract, $0.3 \%$ peptone, $2 \%$ agar, $w / v)$, on SB-agar plates $(3.2 \%$ peptone, $2 \%$ yeast extract, $0.5 \% \mathrm{NaCl}, 2 \%$ agar) and on MRS agar (Scharlau; $\mathrm{g} / \mathrm{l}$ : Peptone proteose 10.00, Meat extract 8.00, Yeast extract $4.00, \mathrm{D}(+)$-Glucose 20.00 , Sodium acetate 5.00 , Triammonium citrate 2.00 , Magnesium sulfate 0.20 , Manganese sulfate 0.05 , Dipotassium phosphate 2.00 , Polysorbate 801.00 , Agar 14.0) incubated at $25^{\circ} \mathrm{C}$. MRS agar is used to grow Lactobacilli; Acetobacter can also grow on this medium but forms smaller colonies. The opposite size distribution can be observed on mannitol plates which is used mainly to grow Acetobacter species. Feeding of bacteria to flies was achieved in both lines kept either at $29^{\circ} \mathrm{C}$ or at $20^{\circ} \mathrm{C}$ by adding $0.1 \mathrm{ml}$ of the formerly isolated Lactobacillus or Acetobacter species at an optical density of 1 at $600 \mathrm{~nm}$ directly to the surface of the food. Clearly distinct bacterial colonies from mannitol-agar plates were pinched and suspended in the PCR-premix for colony PCR. The primers specific for $16 S$ rRNA were 8FE (5'-AGA GTT TGA TCM TGG CTC AG-3') and 1492R (5'-GGM TAC CTT GTT ACG ACT T-3') [13]. ITS1 (TCCGTAGGTGAACCTGCGG) and ITS4 (TCCTCCGCTTATTGATATGC) primers were used for fungal DNA amplification [35]. The products were checked on agarose gel and outsourced for sequencing by Macrogen (Seoul, R. Korea) or to Eurofins MWG Operon (Ebersberg, F.R. Germany).

\section{TUNEL-labeling, ATT::GFP expression, immuno- histochemistry}

Terminal deoxiribonucleotide transferase-mediated dUTP-fluoresceine conjugate nick end labeling (TUNEL) was achieved by in situ cell death detection kit (Roche) as recommended. Embryos of mutant and control flies were incubated at $20^{\circ} \mathrm{C}$ or $29^{\circ} \mathrm{C}$ and L3-stage larvae collected. Nuclei in the dissected guts were counter-stained by $1 \mu \mathrm{g} / \mathrm{ml}$ 4',6-diamidino-2phenylindole (DAPI).

Equimolar mixture of anti-integrin PS I alpha and PSII alpha monoclonal antibodies (mouse, Developmental Studies Hybridoma Bank) were utilized for integrin staining. Mouse monoclonal anti-COL4A1 antibody was raised against the peptide ATGAGSIQDS (29-38) by Creative Ltd., Szeged, Hungary. Primary mouse antibodies were visualized by antimouse Alexa Fluor 488-conjugated secondary antibody (Invitrogen, Life Technology). Laminin gamma 1 polyclonal antibody (rabbit, Abcam) treatment was followed AlexaFluor 568-conjugated anti-rabbit secondary antibody. Experimental procedures described [8] were followed.

Freshly hatched control and mutant adults expressing the Att:GFP transgene were incubated at permissive and restrictive temperatures for three days, guts were dissected. Labellings were visualized by fluorescence microscopy using GFP, FITC or DAPI filters. 


\section{Determination of oxidant concentrations}

Fifty adult flies were homogenized in $450 \mu$ l volume of ice-cold phosphate buffered saline solution by means of a plastic homogenizer immersed in an ice water bath, centrifuged at $17,000 \mathrm{~g}$ for $15 \mathrm{~min}$ at $4{ }^{\circ} \mathrm{C}$, and the clear supernatants were used for $\mathrm{H}_{2} \mathrm{O}_{2}$, $\mathrm{ONOO}^{-}$and protein analysis. The quantity of proteins was determined with Folin-Ciocalteu reagent, using bovine serum albumin as standard [36]. Spectrophotometric measurements were carried out with GENESYS 10S UV-Vis spectrophotometers (Thermo Scientific). For the assay of $\mathrm{H}_{2} \mathrm{O}_{2}, 0.05 \mathrm{mg} / \mathrm{mL}$ horseradish peroxidase and $0.1 \mathrm{mg} / \mathrm{mL}$ o-dianisidine in sodium phosphate buffer $(100 \mathrm{mM}, \mathrm{pH} 6.5)$ was used. The $\mathrm{H}_{2} \mathrm{O}_{2}$ concentration was determined spectrophotometrically at $400 \mathrm{~nm}$ and was calculated in $\mathrm{nmol} / \mathrm{mg}$ protein units [37]. $\mathrm{ONOO}^{-}$was assayed by diluting samples in $1 \mathrm{M} \mathrm{NaOH}(60: 1)$ and measuring the increase in absorbance at $302 \mathrm{~nm}$. As a control, samples were added to $100 \mathrm{mM}$ potassium phosphate (pH 7.4) (60:1). The decrease in absorbance was measured at neutral $\mathrm{pH}$ as $\mathrm{ONOO}^{-}$decomposes [38]. $\mathrm{ONOO}^{-}$concentration was expressed in $\mathrm{nmol} / \mathrm{mg}$ protein units.

Supplementary data to this article can be found online at http://dx.doi.org/10.1016/j.matbio.2015.09. 002.

\section{Disclosure}

The authors have no conflicting interests.

\section{Acknowledgments}

This research was supported by the Hungarian Scientific Research Fund OTKA, contract nr. NN 108283 to M.M. and by the European Union and the State of Hungary, co-financed by the European Social Fund in the framework of TÁMOP-4.2.4.A/ 2-11/1-2012-0001 'National Excellence Program' to M.K. The assistance of Prof. A.H. Meijer (Leiden Univ.) during the development of this manuscript is cordially acknowledged.

Received 10 April 2015; Received in revised form 7 September 2015; Accepted 7 September 2015 Available online 10 September 2015

Keywords:

Type IV collagen; Drosophila; Immunity; Gut epithelia

\section{References}

[1] M.A. Morrissey, D.R. Sherwood, An active role for basement membrane assembly and modification in tissue sculpting, J. Cell Sci. 128 (2015) 1661-1668.

[2] A.C. Alport, Hereditary familial congenital haemorrhagic nephritis, Br. Med. J. 1 (1927) 504-506.

[3] K. Tryggvason, J. Zhou, S.L. Hostikka, T.B. Shows, Molecular genetics of Alport syndrome, Kidney Int. 43 (1993) 38-44.

[4] D.B. Gould, F.C. Phalan, G.J. Breedveld, S.E. van Mil, R.S. Smith, J.C. Schimenti, et al., Mutations in Col4a1 cause perinatal cerebral hemorrhage and porencephaly, Science 308 (2005) 1167-1171.

[5] E. Plaisier, O. Gribouval, S. Alamowitch, B. Mougenot, C. Prost, M.C. Verpont, et al., COL4A1 mutations and hereditary angiopathy, nephropathy, aneurysms, and muscle cramps, N. Engl. J. Med. 357 (2007) 2687-2695.

[6] C. Labelle-Dumais, D.J. Dilworth, E.P. Harrington, M. de Leau, D. Lyons, Z. Kabaeva, et al., COL4A1 mutations cause ocular dysgenesis, neuronal localization defects, and myopathy in mice and Walker-Warburg syndrome in humans, PLoS Genet. 7 (2011), e1002062.

[7] D.S. Kuo, C. Labelle-Dumais, D.B. Gould, COL4A1 and COL4A2 mutations and disease: insights into pathogenic mechanisms and potential therapeutic targets, Hum. Mol. Genet. 21 (2012) R97-110.

[8] I. Kelemen-Valkony, M. Kiss, J. Csiha, A. Kiss, U. Bircher, J. Szidonya, et al., Drosophila basement membrane collagen col4a1 mutations cause severe myopathy, Matrix Biol. 31 (2012) 29-37.

[9] I. Kelemen-Valkony, M. Kiss, K. Csiszar, M. Mink, Inherited Myopathies, Nova Publishers, 2012 1-40.

[10] L.V. Hooper, J.I. Gordon, Commensal host-bacterial relationships in the gut, Science 292 (2001) 1115-1118.

[11] P. Tzou, S. Ohresser, D. Ferrandon, M. Capovilla, J.M. Reichhart, B. Lemaitre, et al., Tissue-specific inducible expression of antimicrobial peptide genes in Drosophila surface epithelia, Immunity 13 (2000) 737-748.

[12] R.T. Jones, L.G. Sanchez, N. Fierer, A cross-taxon analysis of insect-associated bacterial diversity, PLoS One 8 (2013), e61218.

[13] J.-H.H. Ryu, S.-H.H. Kim, H.-Y.Y. Lee, J.Y. Bai, Y.-D.D. Nam, J.-W.W. Bae, et al., Innate immune homeostasis by the homeobox gene caudal and commensal-gut mutualism in Drosophila, Science 319 (2008) 777-782.

[14] S.W. Roh, Y.-D.D. Nam, H.-W.W. Chang, K.-H.H. Kim, M.-S.S. Kim, J.-H.H. Ryu, et al., Phylogenetic characterization of two novel commensal bacteria involved with innate immune homeostasis in Drosophila melanogaster, Appl. Environ. Microbiol. 74 (2008) 6171-6177.

[15] E.-M.M. Ha, C.-T.T. Oh, Y.S. Bae, W.-J.J. Lee, A direct role for dual oxidase in Drosophila gut immunity, Science 310 (2005) 847-850.

[16] B. Lemaitre, J. Hoffmann, The host defense of Drosophila melanogaster, Annu. Rev. Immunol. 25 (2007) 697-743.

[17] S. Panayidou, Y. Apidianakis, Regenerative inflammation: lessons from Drosophila intestinal epithelium in health and disease, Pathogens 2 (2013) 209-231.

[18] M. Rera, S. Bahadorani, J. Cho, C.L. Koehler, M. Ulgherait, J.H. Hur, et al., Modulation of longevity and tissue homeostasis by the Drosophila PGC-1 homolog, Cell Metab. 14 (2011) 623-634. 
[19] M. Rera, R.I. Clark, D.W. Walker, Intestinal barrier dysfunction links metabolic and inflammatory markers of aging to death in Drosophila, Proc. Natl. Acad. Sci. U. S. A. 109 (2012) 21528-21533.

[20] F. Bonnay, E. Cohen-Berros, M. Hoffmann, S.Y. Kim, G.L. Boulianne, J.A. Hoffmann, et al., Big bang gene modulates gut immune tolerance in Drosophila, Proc. Natl. Acad. Sci. U. S. A. 110 (2013) 2957-2962.

[21] O. Puig, M.T. Marr, M.L. Ruhf, R. Tjian, Control of cell number by Drosophila FOXO: downstream and feedback regulation of the insulin receptor pathway, Genes Dev. 17 (2003) 2006-2020.

[22] N.A. Broderick, N. Buchon, B. Lemaitre, Microbiota-induced changes in Drosophila melanogaster host gene expression and gut morphology, MBio. 5 (2014) (e01117-14).

[23] N.-H.H. Choi, J.-G.G. Kim, D.-J.J. Yang, Y.-S.S. Kim, M.-A.A. Yoo, Age-related changes in Drosophila midgut are associated with PVF2, a PDGF/VEGF-like growth factor, Aging Cell 7 (2008) 318-334.

[24] L. Sorokin, The impact of the extracellular matrix on inflammation, Nat. Rev. Immunol. 10 (2010) 712-723.

[25] B. Altincicek, A. Vilcinskas, Metamorphosis and collagen-IVfragments stimulate innate immune response in the greater wax moth, Galleria mellonella, Dev. Comp. Immunol. 30 (2006) 1108-1118.

[26] B. Altincicek, A. Berisha, K. Mukherjee, B. Spengler, A. Römpp, A. Vilcinskas, Identification of collagen IV derived danger/alarm signals in insect immunity by nanoLC-FTICR MS, Biol. Chem. 390 (2009) 1303-1311.

[27] R.O. Hynes, Q. Zhao, The evolution of cell adhesion, J. Cell Biol. 150 (2000) F89-F96.

[28] J. Molnar, Z. Ujfaludi, S.F. Fong, J.A. Bollinger, G. Waro, B. Fogelgren, et al., Drosophila lysyl oxidases dmloxl-1 and dmloxl-2 are differentially expressed and the active DmLOXL-1 influences gene expression and development, J. Biol. Chem. 280 (2005) 22977-22985.

[29] J. Molnar, K.S. Fong, Q.P. He, K. Hayashi, Y. Kim, S.F. Fong, et al., Structural and functional diversity of lysyl oxidase and the LOX-like proteins, Biochim. Biophys. Acta 1647 (2003) 220-224.

[30] J.E. Linder, K.A. Owers, D.E. Promislow, The effects of temperature on host-pathogen interactions in D. melanogaster: who benefits? J. Insect Physiol. 54 (2008) 297-308.

[31] N. Buchon, N.A. Broderick, S. Chakrabarti, B. Lemaitre, Invasive and indigenous microbiota impact intestinal stem cell activity through multiple pathways in Drosophila, Genes Dev. 23 (2009) 2333-2344.

[32] S.C. Shin, S.-H.H. Kim, H. You, B. Kim, A.C. Kim, K.-A.A. Lee, et al., Drosophila microbiome modulates host developmental and metabolic homeostasis via insulin signaling, Science 334 (2011) 670-674.

[33] F.L. Connor, C. Di Lorenzo, Chronic intestinal pseudoobstruction: assessment and management, Gastroenterology 130 (2006) S29-S36.

[34] E. Pöschl, U. Schlötzer-Schrehardt, B. Brachvogel, K. Saito, Y. Ninomiya, U. Mayer, Collagen IV is essential for basement membrane stability but dispensable for initiation of its assembly during early development, Development 131 (2004) 1619-1628.

[35] T. White, T. Bruns, S. Lee, J. Taylor, M. Innis, D. Gelfand, et al., Amplification and Direct Sequencing of Fungal Ribosomal RNA Genes for Phylogenetics, Academic Press, San Diego, 1990 315-322.

[36] O.H. LOWRY, N.J. ROSEBROUGH, A.L. FARR, R.J. RANDALL, Protein measurement with the Folin phenol reagent, J. Biol. Chem. 193 (1951) 265-275.

[37] E. Villegas, S. Gilliland, Hydrogen peroxide production by lactobacillus Delbrueckiisubsp. Lactis I at $5{ }^{\circ} \mathrm{C}$, J. Food Sci. 63 (1998) 1070-1074.

[38] R.E. Huie, S. Padmaja, The reaction of no with superoxide, Free Radic. Res. Commun. 18 (1993) 195-199.

[39] P. Tzou, S. Ohresser, D. Ferrandon, M. Capovilla, J.M. Reichhart, B. Lemaitre, J.A. Hoffmann, J.L. Imler, Tissuespecific inducible expression of antimicrobial peptide genes in Drosophila surface epithelia, Immunity 13 (5) (2000) 737-748. 


\section{Article III.}

Altered stress fibres and integrin expression in the Malpighian epithelium of Drosophila type IV collagen mutants

$\underline{\text { András A. Kiss }}{ }^{\mathrm{a}}$, NikolettaPopovics $^{\mathrm{a}}$, GáborSzabóa ${ }^{\mathrm{a}}$ Katalin Csiszár ${ }^{\mathrm{b}}$, Mátyás Mink ${ }^{\mathrm{a}}$,

Data in Brief (2016)

https://doi.org/10.1016/j.dib.2016.03.059

MTMT azonosító: 3056130

IF: $0(\mathbf{Q} 2)$ 


\title{
Data article
}

\section{Altered stress fibers and integrin expression in the Malpighian epithelium of Drosophila type IV collagen mutants}

\author{
András A. Kiss ${ }^{a}$, Nikoletta Popovics ${ }^{a}$, Gábor Szabó ${ }^{a}$, \\ Katalin Csiszár ${ }^{\mathrm{b}}$, Mátyás Mink ${ }^{\mathrm{a}, *}$ \\ a Department of Genetics, University of Szeged, Középfasor 52, H-6726 Szeged, Hungary \\ b John A. Burns School of Medicine, University of Hawaii, 1960 East West Road, Honolulu, HI 96822, USA
}

\section{A R T I C L E I N F O}

\section{Article history:}

Received 1 December 2015

Received in revised form

8 March 2016

Accepted 15 March 2016

Available online 19 March 2016

Keywords:

col4a1 mutation

Integrin misexpression

Stress fibers

\begin{abstract}
A B S T R A C T
Basement membranes (BMs) are highly specialized extracellular matrices (ECMs) that provide support and polarization cues for epithelial cells. Proper adhesion to the BM is pivotal in epithelial cell function and survival. Type IV collagens are the predominant components of all types of BMs, that form an irregular, polygonal lattice and serve as a scaffold for numerous other BM components and BM-associated cells. Mutations in the ubiquitous human BM components COL4A1 and COLAA2 cause a multisystem disorder involving nephropathy. Affected patients develop renal dysfunction and chronic kidney failure with or without hematuria. Mouse Col4a1 and Col4a2 mutants recapitulate the human symptoms. In vertebrates, excretion is accomplished by the kidneys and by the Malpighian tubules in insects, including the fruit fly Drosophila. Our present results with dominant, temperature-sensitive mutation of the Drosophila col4a1 gene demonstrate altered integrin expression and amplified effects of mechanical stress on the Malpighian epithelial cytoskeleton.
\end{abstract}

c 2016 The Authors. Published by Elsevier Inc. This is an open access article under the CC BY license (http://creativecommons.org/licenses/by/4.0/).

DOI of original article: http://dx.doi.org/10.1016/j.matbio.2015.09.002

* Corresponding author. Tel.: + 3662 544269; fax: + 3662544651.

E-mail address: mink@bio.u-szeged.hu (M. Mink). 


\section{Specifications Table}

$\begin{array}{ll}\text { Subject area } & \text { Biology } \\ \text { More specific subject area } & \text { Genotype-phenotype relationship in Drosophila type IV collagen mutants } \\ \text { Type of data } & \text { Microscopic images, text file } \\ \text { How data was acquired } & \text { Microscope } \\ \text { Data format } & \text { Raw } \\ \text { Experimental factors } & \text { Immunohistochemistry } \\ \text { Experimental features } & \text { Confocal fluorescence pictures } \\ \text { Data source location } & \text { N.a. } \\ \text { Data accessibility } & \text { N.a. }\end{array}$

\section{Value of the data}

- Mutations in the ubiquitous human basement membrane component COL4A1 cause a systemic disease affecting the brain, muscle, blood vessels, kidneys and eyes.

- While mouse models of these mutations recapture the human phenotype, inherent limitations argue for a versatile and more easily tractable model.

- Mutations within the homologous type IV collagen gene in Drosophila recapitulate pathological elements of the human disease and provide suitable phenotypic markers for therapeutic evaluations.

\section{Data}

We have identified dominant, temperature-sensitive mutations within the Drosophila type IV collagen gene col4a1, the insect homologue of mammalian COL4A1. Similar to their mammalian counterparts, the mutations trigger a systemic phenotype, including severe myopathy, intestinal dysfunction and a robust immune response manifested by overexpression of antimicrobial peptides and excess synthesis of the oxidants hydrogen peroxide and peroxynitrite [1-3]. The Malpighian tubules, the excretory organ of insects, are functionally similar to the mammalian kidneys [4]. The tubules are freely floating within the hemocoel, the openly circulating blood-filled body cavity. We surmised that the mechanical impetus of periodic movements of the insect body that keeps the Malpighian tubules in continuous movement, may also contribute to stress-induced cytoskeletal reorganization in mutant animals.
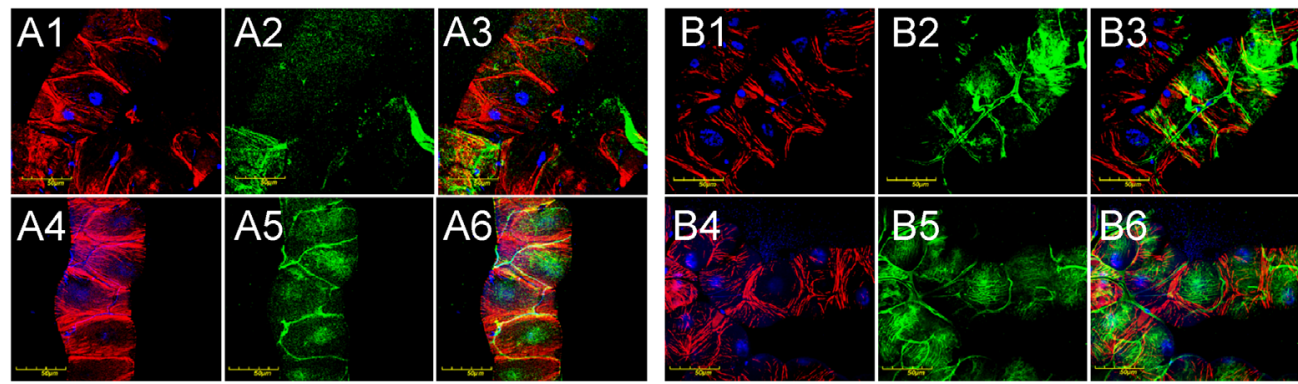

Fig. 1. Malpighan tubules of wild-type (A1-A3 and B1-B3) and mutant (A4-A6 and B4-B6) flies kept at $20^{\circ} \mathrm{C}$ for 3 and 18 days. Red: Actin, blue: DAPI-stained nuclei, green: COL4A1. A1, A4, B1, B4: Actin stained by phalloidin; A2, A5, B2, B5: COL4A1 antibody staining; A3, A6, B3, B6: Merge. Bars equal $50 \mu \mathrm{m}$. 
In order to explore the phenotype of Malpighian tubules associated with mutated Drosophila col4a1 gene, we have chosen a mutant line with the DTS-L3 allele [1-3] as a model, together wild-type OregonR flies. Both lines were incubated at permissive $\left(20^{\circ} \mathrm{C}\right)$ and restrictive $\left(29^{\circ} \mathrm{C}\right)$ temperatures and evaluated following three and eighteen days of incubation. At permissive temperature, both in wild-type and mutant flies actomyosin accumulated in the cortical periphery of epithelial cells irrespective of the incubation times while COL4A1 protein staining showed regular distribution (Fig. 1). At restrictive temperature, epithelial cells of col4a1 mutant developed actin stress fibers visible already at three days of incubation (Fig. 2, A4, white arrow) that became abundant within the cytoplasm by day 18 (Fig. 2, A5). Results were similar to cytoskeletal rearrangement reported for the proximal tubule cells in kidneys of Col4a1 ${ }^{\mathrm{G} 498 \mathrm{~V} / \mathrm{G} 498 \mathrm{~V}}$ homozygous mouse mutants [5]. In the mutants, epithelial cells also expressed less COL4A1 protein both at the third (Fig. 2, A5, yellow arrows) and eighteenth day (Fig. 2, B5, red arrows) of incubation at $29^{\circ} \mathrm{C}$.

Type IV collagens of the BM bind cell-surface integrin receptors as ligands with high affinity and are parts of the cytoskeleton-extracellular matrix axis [6]. The trans-membrane integrins mediate dynamic interactions, including mechano-transduction, between the ECM/BM and the actin cytoskeleton.

To evaluate the effect of altered type IV collagen network on the BM-cytoskeletal axis in mutant animals, we examined the expression and distribution of integrin PS I alpha and PS II alpha subunits.

Immunohistochemistry detected integrins as evenly distributed punctate staining at the surfaces of epithelial cells of Malpighian tubules (Fig. 3) and integrin alpha subunits also co-localized with actin staining as demonstrated by red (actin) and green (integrin) labeling in the merged photomicrographs (Fig. 3, A3, A6, B3, B6, orange) in both mutant and wild-type lines at permissive
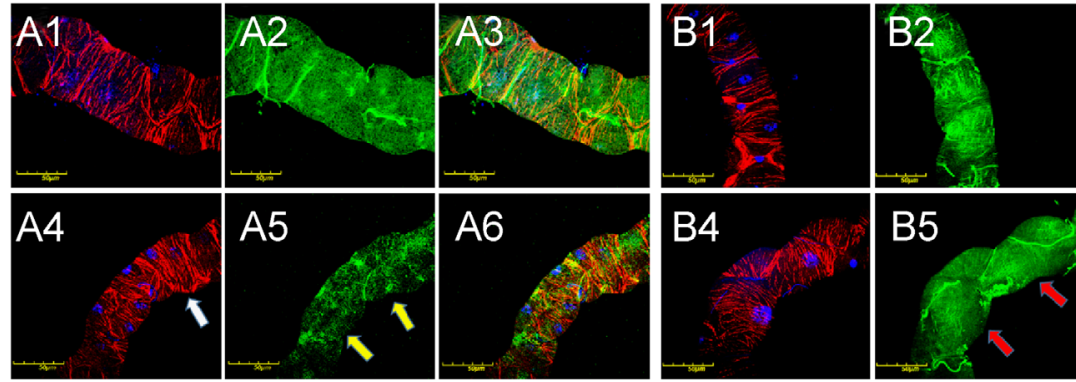

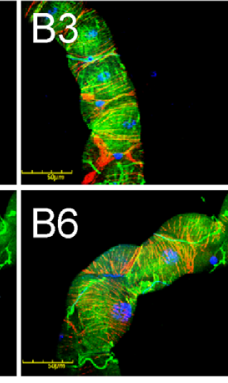

Fig. 2. COL4A1 and stress fiber detection in Malpighan tubules of wild-type (A1-A3 and B1-B3) and mutant (A4-A6 and B4B6) flies incubated at $29^{\circ} \mathrm{C}$ for 3 and 18 days. Red: Actin, blue: DAPI-stained nuclei, green: COL4A1. A1, A4, B1, B4: Actin stained by phalloidin; A2, A5, B2, B5: COL4A1 antibody staining; A3, A6, B3, B6: Merge. Stress fibers appear in mutants following three days incubation at $29^{\circ} \mathrm{C}$ (A4, white arrow) and became more abundant by day 18 (B4); mutant Malpighian tubules express less COL4A1 (A5, yellow arrows, B5, red arrows). Bars equal $50 \mu \mathrm{m}$.
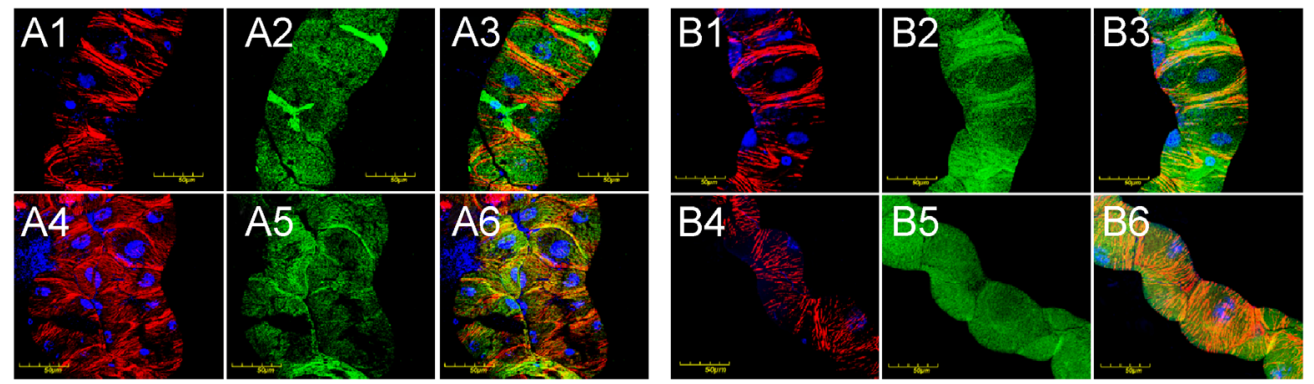

Fig. 3. Integrin detection in Malpighan tubules of wild-type (A1-A3 and B1-B3) and mutant (A4-A6 and B4-B6) flies incubated at $20{ }^{\circ} \mathrm{C}$ for 3 and 18 days. Red: Actin, blue: DAPI-stained nuclei, green: Integrin-staining by anti-PS I alpha and PS II alpha antibodies. Bars equal $50 \mu \mathrm{m}$. 

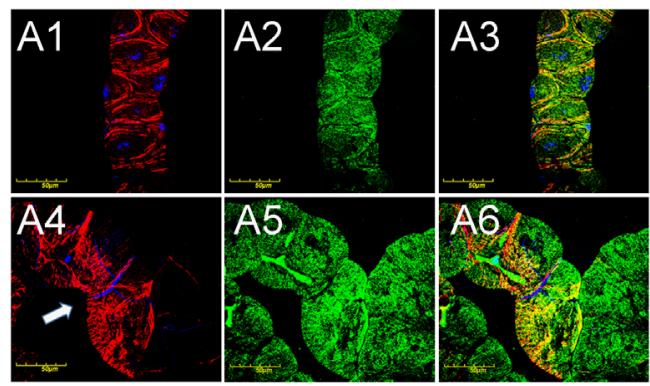
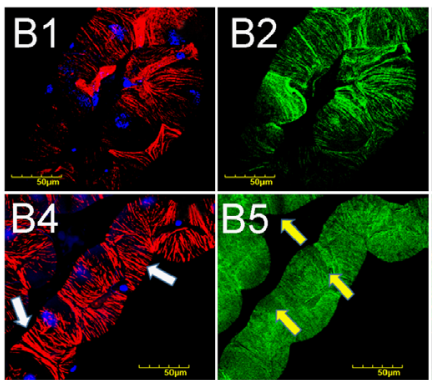

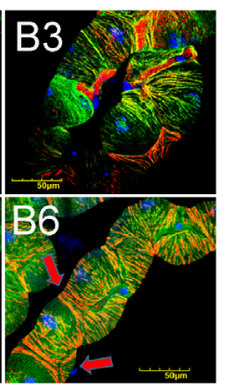

Fig. 4. Altered integrin localization and stress fibers in Malpighan tubules of wild-type (A1-A3 and B1-B3) and mutant (A4-A6 and B4-B6) flies incubated at $29^{\circ} \mathrm{C}$ for 3 and 18 days. Red: Actin, blue: DAPI-stained nuclei, green: Integrin-staining by anti-PS I alpha and PS II alpha antibodies. Stress fibers appeared following three days heat shock (A4, white arrow) and became more abundant by day 18 (B4, white arrows). Alpha integrins co-localized with actin (B3) in wild-type controls whereas in mutants, large areas showed reduced staining for integrins (B5, yellow arrow), furthermore, integrin signals did not co-localize with actin (B6, red arrows). Bars equal $50 \mu \mathrm{m}$.

temperature irrespective of the age of the flies or length of incubation. At elevated temperature, mutant lines developed actin stress fibers by day 3 (Fig. 4, A4, white arrow) that persisted at day 18 (Fig. 4, B4, white arrows) a feature that was not observed in wild-type animals (Fig. 4, A1, B1). In mutants, integrin staining was uneven with areas of minimal expression at day 18 (Fig. 4, B5, yellow arrows). Additionally, integrin and actin connections appeared disrupted as these proteins were no longer co-localized, demonstrated by areas with isolated integrin staining (Fig. 4, B6, red arrows). In wild-type controls, integrin and actin appeared in close proximity supporting the existence of proper cytoskeleton-ECM linkage (Fig. 4, B3). The data collectively demonstrate that col4a1 mutation affects integrin expression, causes irregular accumulation of integrins and an increase in stress fibers within the epithelial cells of Malpighian tubules due to impaired mechano-transduction. These phenotypic changes in Drosophila col4a1 mutant model represent cellular markers suitable for rapid and costeffective evaluations of targeted therapeutical interventions.

\section{Experimental design, materials and methods}

Mutant and control flies were propagated on yeast-cornmeal-agar medium consisting of nipagine to prevent fungal infections. Malpighian tubules were dissected from carbon-dioxide-anesthetized wild-type and mutant flies, fixed and processed as described [1]. Mouse monoclonal antibody against Drosophila COL4A1 protein was generated using the peptide ATGAGSIQDS (29-38, Creative Ltd, Szeged, Hungary). To visualize integrin dimers consisting of either PS I alpha or PS II alpha subunits, an equimolar mixture of both anti-integrin monoclonal antibodies (mouse, Developmental Studies Hybridoma Bank) were utilized. Primary mouse antibodies were visualized by anti-mouse Alexa Fluor 488-conjugated secondary antibody (Invitrogen, Life Technology). Photomicrographs were taken by an Olympus Fluoview FV1000 confocal laser scanning microscope (Olympus Life Science Europa GmbH, Hamburg, Germany).

\section{Competing interests}

The authors have declared that no competing interest exists. 


\section{Acknowledgments}

This research was supported by the Hungarian Scientific Research Fund OTKA, contract no. NN 108283 to M.M.

\section{Appendix A. Supplementary material}

Supplementary data associated with this article can be found in the online version at http://dx.doi. org/10.1016/j.dib.2016.03.059.

\section{References}

[1] I. Kelemen-Valkony, M. Kiss, J. Csiha, A. Kiss, U. Bircher, J. Szidonya, P. Maróy, G. Juhász, O. Komonyi, K. Csiszár, M. Mink, Drosophila basement membrane collagen col4a1 mutations cause severe myopathy, Matrix Biol. 31 (2012) 29-37.

[2] I. Kelemen-Valkony, M. Kiss, K. Csiszar, Mink Inherited Myopathies, in: H.S. Washington, C.E. Castillo Jimenez (Eds.), Myopathies: New Research, Nova Science Publishers, Hauppauge, 2012, pp. 1-40 (ISBN:978-1-62257-372-1).

[3] M. Kiss, A.A. Kiss, M. Radics, N. Popovics, E. Hermesz, K. Csiszár, M. Mink, Drosophila type IV collagen mutation associates with immune system activation and intestinal dysfunction, Matrix Biol. (2016) (In press).

[4] J.A. Dow, M.F. Romero, Drosophila provides rapid modeling of renal development,function, and disease, Am. J. Physiol. Ren. Physiol. 299 (2010) F1237-F1244.

[5] Zh Chen, T. Migeon, M.-Ch Verpont, M. Zaidan, Y. Sado, D. Kerjaschki, P. Ronco, E. Plaisier, HANAC syndrome Col4a1 mutation causes neonate glomerular hyperpermeability and adult glomerulocystic kidney disease, J. Am. Soc. Nephrol. (2015), http://dx.doi.org/10.1681/ASN.2014121217.

[6] S.A. Wickström, K. Radovanac, R. Fässler, Genetic analyses of integrin signaling, Cold Spring Harb. Perspect. Biol. (2010), http://dx.doi.org/10.1101/cshperspect.a005116. 


\section{Article IV.}

4-Hydroxy-2-nonenal Alkylated and Peroxynitrite Nitrated Proteins Localize to the Fused Mitochondria in Malpighian Epithelial Cells of Type IV Collagen Drosophila Mutants

$\underline{\text { András A. Kiss }}{ }^{1}$, Nikoletta Popovics ${ }^{1}$, Zsolt Boldogkői ${ }^{1}$, Katalin Csiszár ${ }^{2}$ and Mátyás $\operatorname{Mink}^{1}$

BioMed Research International (2018)

https://doi.org/10.1155/2018/3502401

MTMT azonosító: 3326500

IF: $2.197(Q 2)$ 


\title{
4-Hydroxy-2-nonenal Alkylated and Peroxynitrite Nitrated Proteins Localize to the Fused Mitochondria in Malpighian Epithelial Cells of Type IV Collagen Drosophila Mutants
}

\author{
András A. Kiss, ${ }^{1}$ Nikoletta Popovics, ${ }^{1}$ Zsolt Boldogkői, ${ }^{1}$ \\ Katalin Csiszár, ${ }^{2}$ and Mátyás Mink ${ }^{1}$ \\ ${ }^{1}$ Institute of Medical Biology, University of Szeged, Somogyi B. U. 4, 6720 Szeged, Hungary \\ ${ }^{2}$ John A. Burns School of Medicine, University of Hawaii, 1960 East West Road, Honolulu, HI 96822, USA
}

Correspondence should be addressed to Mátyás Mink; mink@bio.u-szeged.hu

Received 4 September 2017; Revised 3 December 2017; Accepted 2 January 2018; Published 30 January 2018

Academic Editor: Daniela Grifoni

Copyright (C) 2018 András A. Kiss et al. This is an open access article distributed under the Creative Commons Attribution License, which permits unrestricted use, distribution, and reproduction in any medium, provided the original work is properly cited.

\begin{abstract}
Background. Human type IV collagenopathy is associated with mutations within the COL $4 A 1$ and to a less extent the COL4A2 genes. The proteins encoded by these genes form heterotrimers and are the highest molar ratio components of the ubiquitous basement membrane. The clinical manifestations of the COL4A1/A2 mutations are systemic affecting many tissues and organs among these kidneys. In order to uncover the cellular and biochemical alterations associated with aberrant type IV collagen, we have explored the phenotype of the Malpighian tubules, the secretory organ and insect kidney model, in col4al collagen gene mutants of the fruit fly Drosophila melanogaster. In Malpighian epithelial cells of col4a1 mutants, robust mitochondrial fusion indicated mutation-induced stress. Immunohistochemistry detected proteins nitrated by peroxynitrite that localized to the enlarged mitochondria and increased level of membrane peroxidation, assessed by the amount of proteins alkylated by 4-hydroxy-2-nonenal that similarly localized to the fused mitochondria. Nuclei within the Malpighian epithelium showed TUNEL-positivity suggesting cell degradation. The results demonstrated that col4a1 mutations affect the epithelia and, consequently, secretory function of the Malpighian tubules and provide mechanistic insight into col4al mutation-associated functional impairments not yet reported in human patients and in mouse models with mutant COL4A1.
\end{abstract}

\section{Introduction}

Basement membranes (BMs) are nanoscale sheets of extracellular matrices that play essential roles in multiple organs including muscle homeostasis, structures, and integrity of the dermal and ocular system, neuromuscular junctions, and blood filtration in the kidneys. The most abundant structural components of BMs include laminins, collagen IV, nidogens, perlecan, and agrin [1]. The ubiquitous mammalian BMs consist of heterotrimeric type IV collagens with $(\mathrm{COL} 4 \mathrm{Al})_{2}$ COL4Al composition. The clinical presentation of patients with COL4A1 mutation is systemic with numerous affected organs and tissues including the eyes, brain, the vascular system, skeletal muscles, and kidneys [2,3]. A distinct form of type IV collagenopathy, Hereditary Angiopathy, Nephropathy, Aneurysms, and Muscle Cramps (HANAC) syndrome, is caused by $\mathrm{N}$-terminal mutations within the COL4A1 gene. The renal manifestation of the same mutations in mouse models includes albuminuria, hematuria, glomerular cysts, and delays in glomerulogenesis and podocyte differentiation [4].

We have identified an allelic series of dominant, temperature-sensitive, antimorph mutations in the cognate col4al gene of the fruit fly, Drosophila melanogaster. The $\mathrm{col}_{4 a 1^{+/-}}$heterozygotes are viable and fertile at permissive temperature of $20^{\circ} \mathrm{C}$ but die at $29^{\circ} \mathrm{C}$. In these mutants, we 
have reported severe myopathy [5], tortuous BM, detachment of the gut epithelial and visceral muscle cells from the BM [6], intestinal dysfunction, overexpression of antimicrobial peptides, and excess synthesis of the oxidants hydrogen peroxide and peroxynitrite [7]. Peroxynitrite, by substituting the hydrogen atom by a nitro $\left(-\mathrm{NO}_{2}\right)$ group adjacent to the hydroxyl group on the aromatic ring of tyrosine, adversely impacts protein functions and can be detected as a speciesindependent antigene [8]. Peroxynitrite can also remove a hydrogen atom from polyunsaturated fatty acids resulting in the formation of aldehydes, conjugated dienes, and hydroperoxyradicals that trigger a free radical chain reaction and membrane lipid damage by lipoperoxidation [9].

The main product of membranous polyunsaturated fatty acid peroxidation is the reactive 4-hydroxy-2-nonenal, HNE [10]. The reactivity of HNE with proteins relies on Michael addiction and, by modifying histidine residues, generates alkyl-conjugated polypeptides also detectable as speciesindependent antigens [11]. As there is no direct laboratory test to estimate lipid peroxidation, measurements of HNE-conjugated protein levels currently serve as surrogates [12]. The bulk of peroxynitrite reacts rapidly with carbon dioxide, present at $\sim 1 \mathrm{mM}$ in cells, forming the unstable product nitrosoperoxycarbonate $\left(\mathrm{ONOOCO}_{2}^{-}\right)$, one-third of which decomposes into carbonate $\left(\mathrm{CO}^{-\bullet}\right)$ and $\mathrm{NO}_{2}{ }^{\bullet}$ radicals and two-thirds into the neutral $\mathrm{NO}_{3}{ }^{-}$and $\mathrm{CO}_{2}$ [13].

Insect Malpighian tubules serve as secretory organs. These renal tubules lack a vascular blood system and float freely in the hemocoel (blood-filled body cavity). The tubules are surrounded by BM and consist of two epithelial cell types, the metabolically active principal and the intercalated stellate cells [14]. The insect renal system is aglomerular, and urine is formed by active transport rather than by selective reabsorption of ultrafiltrate as in vertebrates. While the insect tubule system represents an intermediate towards the glomerular kidney, it fulfills the same basic functions of transport, excretion, and osmoregulation [15].

We have recently shown that the col4al Drosophila mutants develop stress fibers in their Malpighian cells and aberrantly express cell-surface integrin receptors [16]. In the present study, we have extended our research to address altered posttranslational protein modifications by peroxynitrite and 4-hydroxy-2-nonenal in the Malpighian tubules. The col4al mutants demonstrated heavy protein tyrosine nitration and protein-histidine alkylation that localized to the enlarged and fused mitochondria as signs of mitochondrial stress. HNE-protein adducts colocalized with the cytoplasmic membrane that was accompanied by cell degeneration in the tubules performing TUNEL-positivity, collectively suggesting that these aberrant processes are integral parts of col4a1associated pathology.

\section{Materials and Methods}

2.1. Maintenance of Drosophila Strains. Wild-type Oregon flies and col4a1 mutant stock with the DTS-L3 allele were maintained at $20^{\circ} \mathrm{C}$ and $29^{\circ} \mathrm{C}$ on yeast-cornmeal-sucroseagar food, consisting of nipagin to prevent fungal infection.
The mutant stocks were kept heterozygous over the CyRoi balancer chromosome. Malpighian tubules were removed under carbon dioxide anesthesia from adults that were grown at both permissive and restrictive temperature for 14 days. Dissected Malpighian tubules were fixed in 4\% paraformaldehyde dissolved in phosphate buffered saline (PBS) for $10 \mathrm{~min}$, washed three times in PBS, permeabilized for $5 \mathrm{~min}$ in $0,1 \%$ Triton X, dissolved in PBS, and washed three times in PBS. Blocking was achieved in 5\% BSA dissolved in PBS for 1 hour and washed three times in PBS.

2.2. Immunostaining and Antibodies. Nuclei in the dissected Malpighian tubules were counterstained by $1 \mu \mathrm{g} / \mathrm{ml} 4^{\prime}, 6$ diamino-2-phenylindole (DAPI) in $20 \mu \mathrm{l} \mathrm{PBS}, 12 \mathrm{~min}$ in dark. F-actin was stained by 1 unit Texas $\operatorname{Red}^{\mathrm{TM}}-\mathrm{X}$ Phalloidin (ThermoFisher) in $20 \mu \mathrm{l}$ PBS for $20 \mathrm{~min}$. A-Mannopyranosyl and a-glucopyranosyl residues as membrane markers were stained by Concanavalin A, Alexa Fluor ${ }^{\mathrm{TM}} 594$ Conjugate (ThermoFisher) in $20 \mu \mathrm{l}$ PBS for 1 hour. We used $1 \mu \mathrm{l}$ mouse monoclonal anti-3-nitrotyrosine [39B6] (Abcam) in $20 \mu \mathrm{l}$ PBS for 1 hour and stained 4-hydroxynonenal conjugate by $1 \mu \mathrm{l}$ mouse monoclonal anti-4-hydroxynonenal antibody (Abcam) in $20 \mu \mathrm{l}$ PBS for 1 hour. Primary mouse antibodies were visualized by $1 \mu \mathrm{l} \mathrm{F}\left(\mathrm{ab}^{\prime}\right)$ 2-Goat Anti-Mouse IgG $(\mathrm{H}$ + L) Cross Adsorbed Secondary Antibody conjugated with Alexa Fluor 488 (ThermoFisher) in $20 \mu \mathrm{l}$ PBS for 1 hour and $1 \mu \mathrm{l}$ Goat Anti-Mouse IgG $(\mathrm{H}+\mathrm{L})$ Cross Adsorbed Secondary Antibody, Alexa Fluor 350, in $20 \mu \mathrm{l}$ PBS for 1 hour. Mitochondria were visualized by the mitochondrially targeted EYFP (mito-GFP) following appropriate crosses [17].

2.3. Confocal Microscopy. Photomicrographs of the Malpighian tubules were generated by confocal laser scanning fluorescence microscopy (Olympus Life Science Europa $\mathrm{GmbH}$, Hamburg, Germany). Microscope configuration was the following: objective lens: UPLSAPO 60x (oil, NA: 1.35); sampling speed: $8 \mu \mathrm{s} /$ pixel; line averaging: $2 \mathrm{x}$; scanning mode: sequential unidirectional; excitation: $405 \mathrm{~nm}$ (DAPI), $543 \mathrm{~nm}$ (Texas Red), and $488 \mathrm{~nm}$ (Alexa Fluor 488); laser transmissivity: $7 \%$ were used for DAPI, $42 \%$ for Alexa Fluor 488 and $52 \%$ for Texas Red.

2.4. TUNEL-Labelling. Terminal deoxyribonucleotide transferase-mediated dUTP-fluorescein conjugate nick end labelling (TUNEL) was carried out by using the in situ cell death detection kit (Roche) as recommended. Embryos of mutant and control flies were incubated at $20^{\circ} \mathrm{C}$ or $29^{\circ} \mathrm{C}$ and L3-stage larvae collected. Nuclei in the Malpighian tubules were counterstained by $1 \mu \mathrm{g} / \mathrm{ml} 4^{\prime}, 6$-diamidino-2phenylindole (DAPI). Labellings were visualized by a HundWetzlar fluorescence microscope by using FITC or DAPI filters.

\section{Results}

3.1. Heavy Protein Nitration in col4a1 Mutants. We have previously demonstrated that the col4al mutant flies synthesize peroxynitrite at higher concentration as part of their antimicrobial immune response under restrictive conditions [7]. 


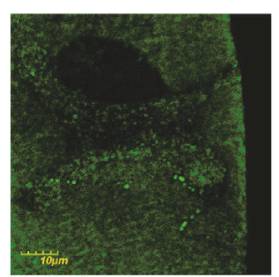

(a)

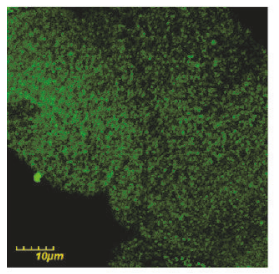

(g)

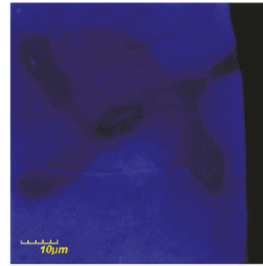

(b)

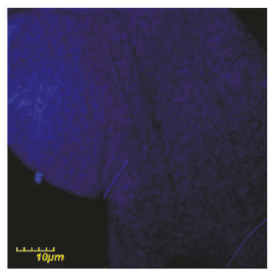

(h)

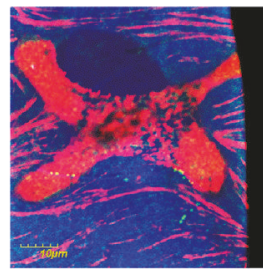

(c)

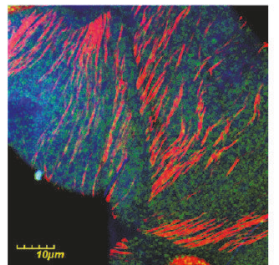

(i)

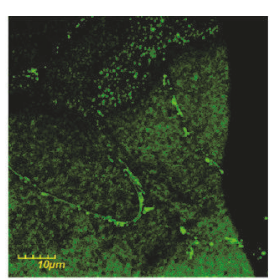

(d)

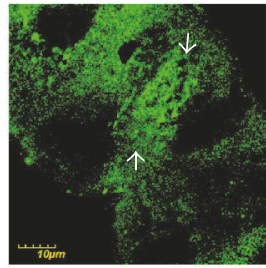

(j)

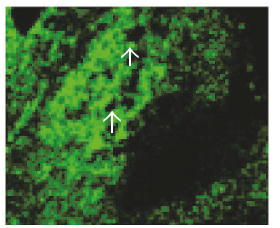

(m)

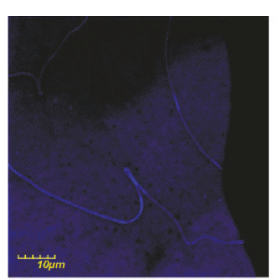

(e)

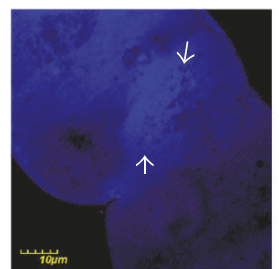

(k)

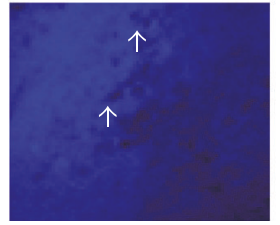

(n)

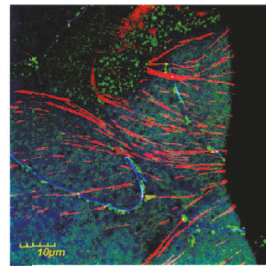

(f)

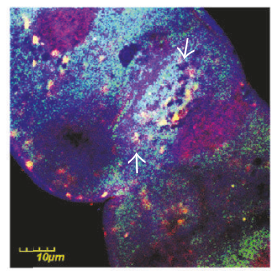

(l)

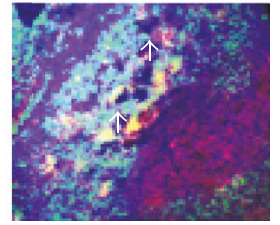

(o)

FIgURE 1: Protein nitration in Malpighian epithelial cells. Color code: mitochondria, green; nitrated proteins, blue; actin, red. (a) Wild-type flies incubated at $20^{\circ} \mathrm{C}$, mitochondria, (b) nitrated proteins, and (c) merge. Note a stellate cell in (c). ((d), (e), (f)) Wild-type flies incubated at $29^{\circ} \mathrm{C}$ and displayed in the same order. ((g)-(i)) Mutant flies, incubated at $20^{\circ} \mathrm{C}$, and $((\mathrm{j})-(\mathrm{l}))$ mutant flies, incubated at $29^{\circ} \mathrm{C}$. Photomicrographs are displayed in the same order as in the upper row. Localization of nitrated proteins to mitochondria is shown in (c), (i), (f), and (l). Uneven distribution and fusion of mitochondria are demonstrated in (j). White arrows in (j), (k), and (l) pointing the region displayed in higher magnification in $(\mathrm{m}),(\mathrm{n})$, and (o). White arrows in $(\mathrm{m}),(\mathrm{n})$, and (o) showing regions with no/few mitochondria and the lack of staining, demonstrating localization to mitochondria with nitrated proteins indirectly.

Peroxynitrite is produced by the diffusion-driven reaction of nitric oxide (NO) in the presence of oxidants such as the mitochondrial-derived radical superoxide anion, $\mathrm{O}_{2}{ }^{--}$. The sources of $\mathrm{NO}$ are at extramitochondrial sites and the dissolved gas diffuses into mitochondria, reacts with $\mathrm{O} 2^{\circ-}$, and disrupts protein functions by protein tyrosine nitration [18]. We therefore expected accumulation nitrated proteins in the mitochondria of col4al mutant flies following incubation at $29^{\circ} \mathrm{C}$.

We did not observe gross alterations in the Malpighian tubules of the mutants compared to control flies; mitochondria were distributed evenly in the cytoplasm and the fluorescent light intensities used to record nitrated proteins in the mutants were comparable to the control animals (Figures 1(a)-1(c) and Figures 1(g)-1(i)), following incubation at permissive condition. However, under restrictive conditions $\left(29^{\circ} \mathrm{C}\right)$, we noted marked differences in the Malpighian tubules of mutant flies. While mitochondria in the epithelial cells of wild-type Malpighian tubules remained evenly distributed with no shape alteration at this temperature (Figure 1(d)), in mutants, mitochondrial fusion and uneven distribution were observed (Figure 1(j)). The level of nitrated proteins was remarkably higher in mutants in comparison with the control (Figure $1(\mathrm{k})$ versus Figure $1(\mathrm{e})$ ) and these signals localized to the mitochondria (Figures 1(f) and 1(l)).

3.2. High Levels of Alkylated Proteins in the Mutants. The level of lipid peroxidation was determined indirectly by the accumulation of HNE-protein adducts. Results showed comparable amounts of alkylated proteins in the epithelial cells of mutant Malpighian tubules at permissive condition (Figure 2(b) versus Figure 2(h)), and the appearance of mitochondria remained unaffected in both mutants and controls under these conditions (Figure 2(a) versus Figure $2(\mathrm{~g}))$. In mutants under restrictive conditions $\left(29^{\circ} \mathrm{C}\right)$, uneven distribution and fusion of mitochondria occurred (Figure 2(j) versus Figure 2(d)), the mutants produced more HNE-protein adducts (Figure 2(k) versus Figure 2(e)), and the alkylated proteins localized to mitochondria (Figures $2(\mathrm{f})$ and 2(l)).

3.3. Protein-HNE Adducts Associate with Cytoplasmic Membrane. We next explored the involvement of the cytoplasmic membrane in col4al-associated pathology. We recorded numerous alkylation sites in form of punctate staining in colocalization with the cytoplasmic side of the membrane 


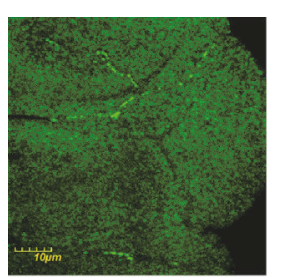

(a)

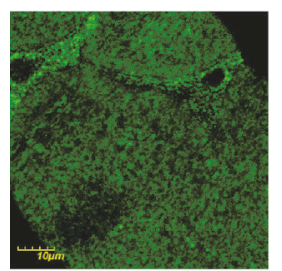

(g)

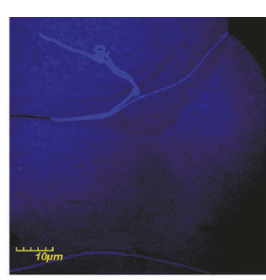

(b)

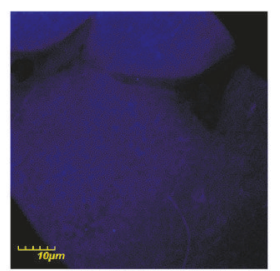

(h)

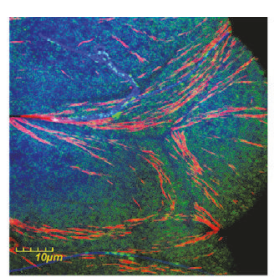

(c)

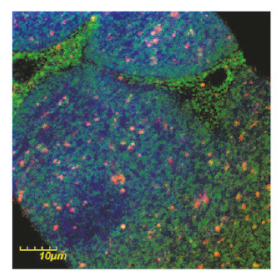

(i)

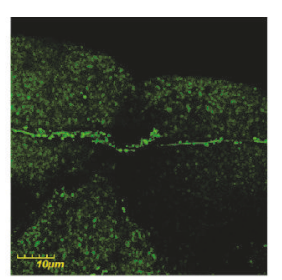

(d)

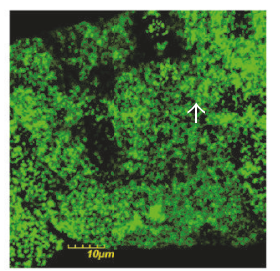

(j)

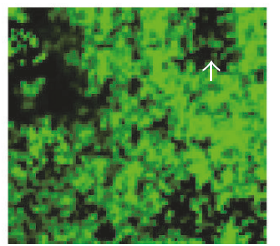

(m)

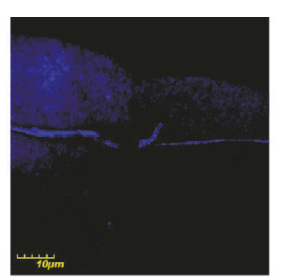

(e)

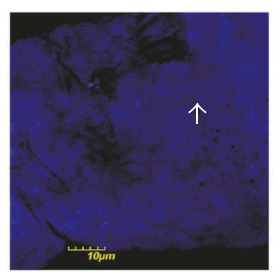

(k)

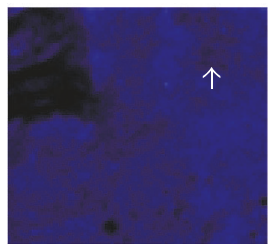

(n)

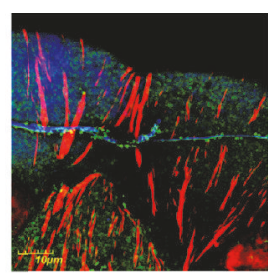

(f)

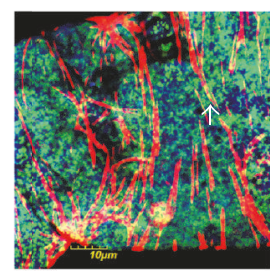

(1)

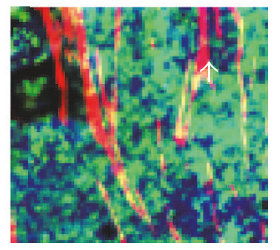

(o)

Figure 2: Protein-HNE adducts in Malpighian epithelium. Color code: mitochondria, green; protein-HNE adducts, blue; actin, red. (a) Mitochondria of wild-type flies incubated at $20^{\circ} \mathrm{C}$, (b) protein-HNE adducts, and (c) merge. ((d), (e), (f)) Wild-type flies incubated at $29^{\circ} \mathrm{C}$, shown in the same order. ((g)-(i)) Mutant flies, incubated at $20^{\circ} \mathrm{C}$, and $((\mathrm{j})-(\mathrm{l}))$ mutant flies, incubated at $29^{\circ} \mathrm{C}$. The order of photomicrographs is as in upper row. Note mitochondrial fusion in ( $\mathrm{j}$ ) and actin stress fibers in (l). White arrow in (j), (k), and (l) showing the portion displayed in higher magnification in (m), (n), and (o). White arrow in $(\mathrm{m}),(\mathrm{n})$, and (o): point regions with no/few mitochondria and the lack of staining, demonstrating localization of alkylated proteins to mitochondria indirectly.

and apparent perinuclear accumulation in the Malpighian epithelial cells in the mutants at permissive conditions $\left(20^{\circ} \mathrm{C}\right)$ (Figures $3(\mathrm{e})$ and $3(\mathrm{f})$ ). This staining pattern was amplified upon shift to restrictive temperature $\left(29^{\circ} \mathrm{C}\right)$ and the HNE-conjugated proteins appeared within the cytoplasmic membrane indicating direct membrane damage by lipid peroxidation (Figures $3(\mathrm{~g})$ and $3(\mathrm{~h})$ ). In the control flies the cytoplasmic membrane remained intact and protein-HNE adducts appeared in the vicinity of the membrane at both permissive and restrictive conditions (Figures 3(a)-3(d)).

3.4. Cell Degeneration Detected by TUNEL-Positivity. The epithelial cells of the Malpighian tubules proved to be TUNEL-positive in mutants at $29^{\circ} \mathrm{C}$ (Figures $4(\mathrm{~d})-4(\mathrm{f})$ ), but not at $20^{\circ} \mathrm{C}$ (Figures $4(\mathrm{a})-4(\mathrm{c})$ ). These observations further supported our earlier observations of cell death affecting multiple tissues in col4al mutants.

\section{Discussion}

Drosophila models provide useful tools for determining the pathomechanistic details, functional alterations, and some of the genotype-phenotype correlations of human monogenic disorders [19] including mutations associated with disorders of the kidneys as some of the human genes known to be associated with inherited nephrotic syndromes play conserved roles in renal functions from flies to humans [20]. There are nephrotic manifestations of human COL4A1 mutations of the Hereditary Angiopathy, Nephropathy, Aneurysms, and Muscle Cramps (HANAC) syndrome [21] and recent research revealed glomerular hyperpermeability and adult onset glomerulocystic kidney disease in association with COL4A1 mutations [4]. Some of the mechanistic elements in context of type IV collagen mutations, such as oxidative stress, have also been demonstrated [22]. However, evidence for chronic inflammation and posttranslational protein modifications are scarce and so far demonstrated only in Drosophila col4al mutants $[7,16]$.

Mitochondrial fusion occurs under situations of cellular stress. Merging of the contents of partially damaged mitochondria is interpreted as a complementation mechanism rescuing impaired organelles and function [23]. Our prior results demonstrated signs of cellular stress in the form of actin stress fibers in the Malpighian epithelial cells of col4al mutant Drosophila [16]. Results of the current study show that mutation-associated stress induced mitochondrial hyperfusion also occurs under restrictive condition with the enlarged organelles unevenly distributed within cells resulting either 


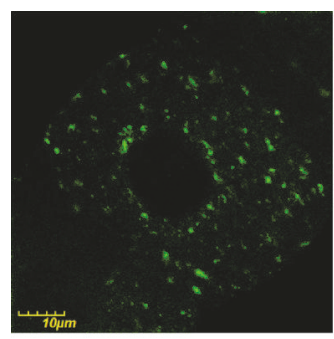

(a)

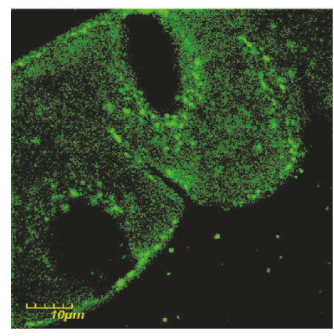

(e)

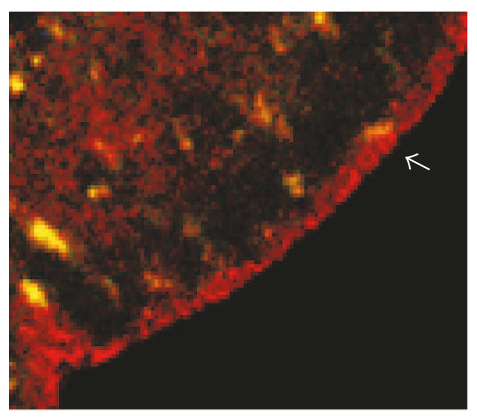

(i)

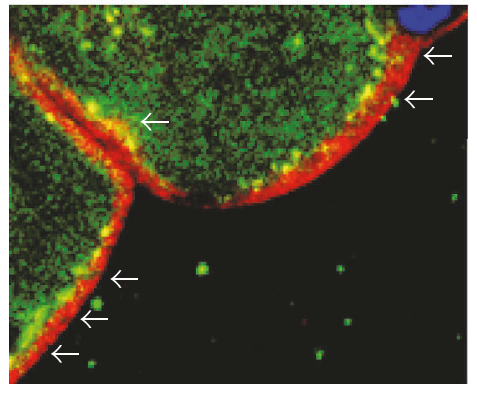

(j)

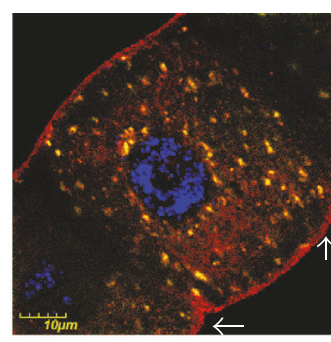

(b)

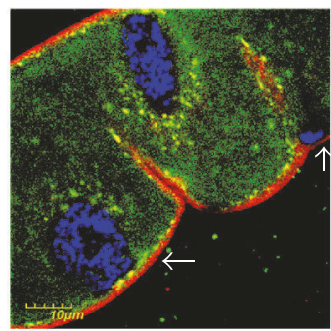

(f)

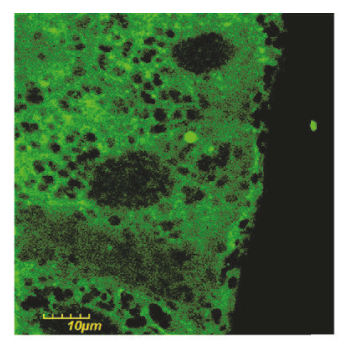

(c)

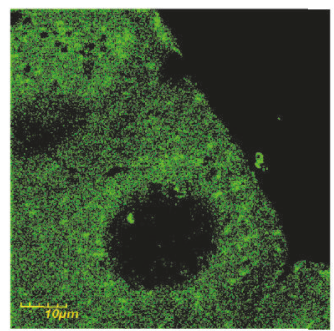

(g)

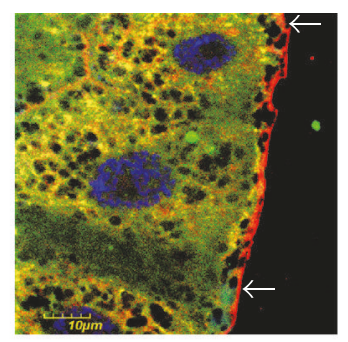

(d)

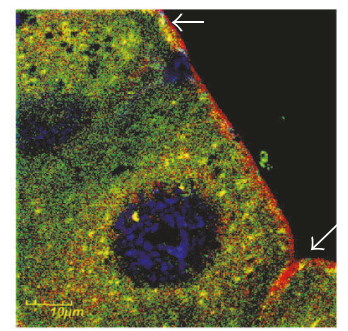

(h)

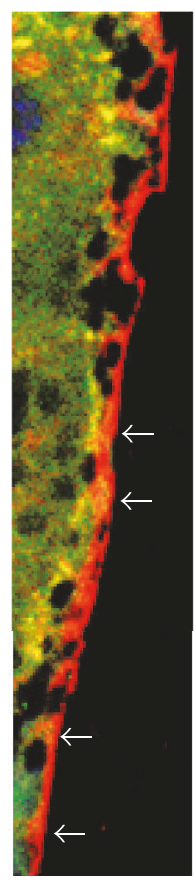

(k)

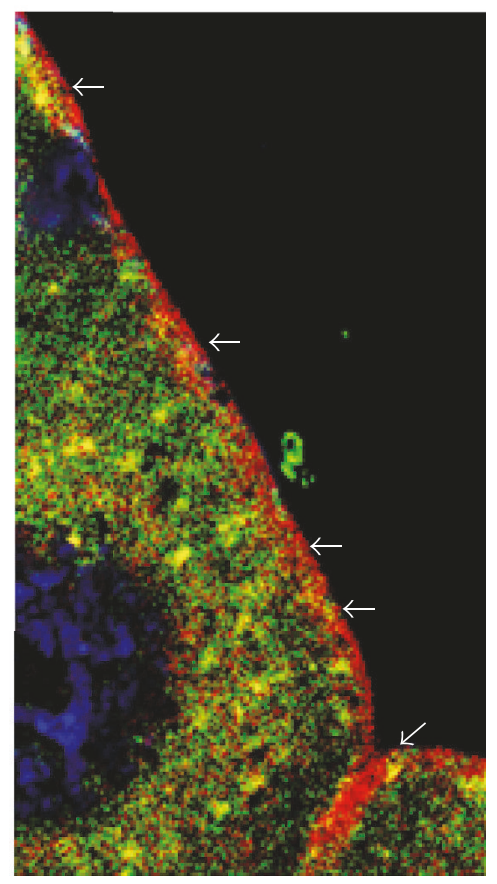

(1)

Figure 3: Cytoplasmic membrane-associated HNE-modified proteins in mutants. Color code: protein-HNE adducts, green; cytoplasmic membrane red; nuclei, blue. (a) Protein-HNE adducts in wild-type flies incubated at $20^{\circ} \mathrm{C}$. (b) Overlay with membrane staining. (c) ProteinHNE adducts in wild-type flies incubated at $29^{\circ} \mathrm{C}$. (d) Merged with membrane staining. ((e), (f), (g), (h)) Representative mutant, incubated at 20 or $29^{\circ} \mathrm{C}$ presented in the same order as in upper row. White arrows in (b), (d), (f), and (h). Regions presented in higher magnification in (i), (j), (k), and (l), respectively. White arrows in (i), (j), (k), and (l) show association of the cytoplasmic membrane with alkylated proteins. Note the notorious infiltration of HNE-modified proteins into the membrane in (l), which occurs at a less extent in (k). The membrane of wild-type animals is free of alkylated proteins (i), while they associate closely with the membrane in the mutant (j), incubated at permissive temperature. 


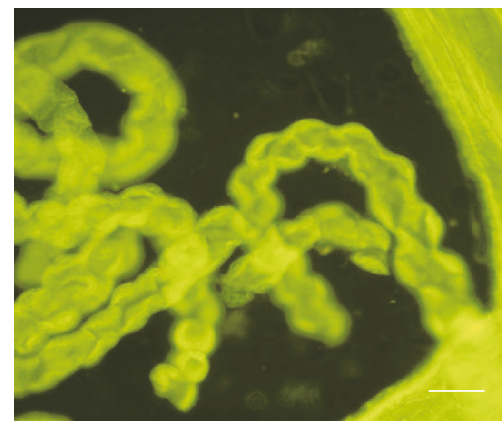

(a)

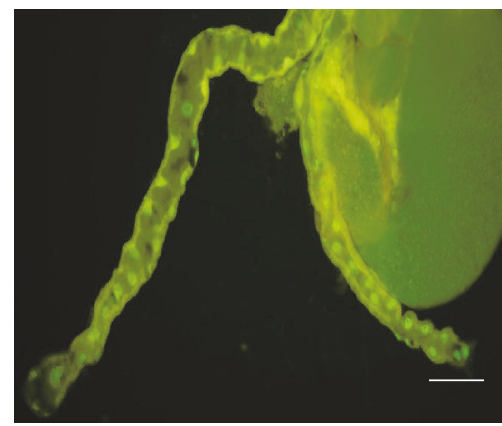

(d)

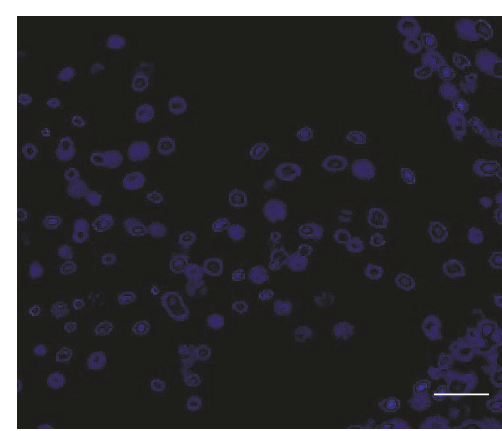

(b)

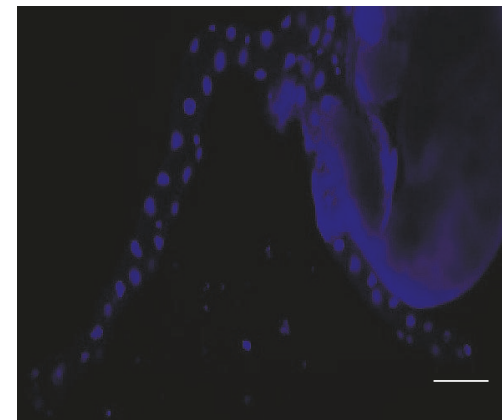

(e)

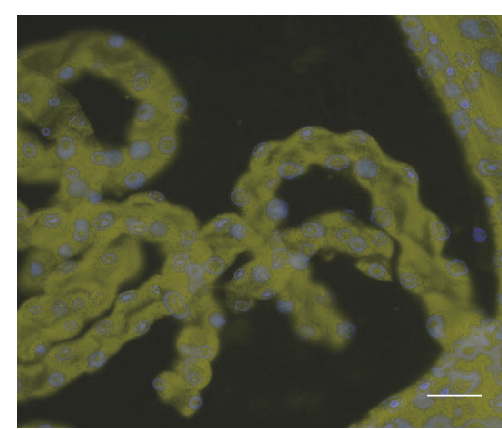

(c)

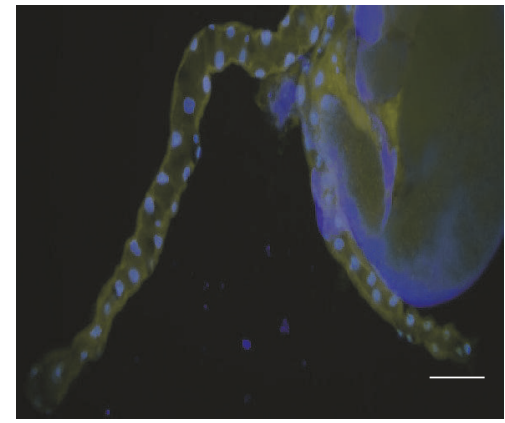

(f)

FIGURE 4: Fluorescence micrographs demonstrating TUNEL-positivity in Malpighian tubules. (a) TUNEL-staining of a Malpighian tubule of col4a1 ${ }^{+/-}$L3-larva incubated at $20^{\circ} \mathrm{C}$; (b) DAPI-staining; (c) merge, tubules appearing TUNEL-negative. (d) TUNEL-positive Malpighian tubule of a col4al ${ }^{+/-}$L3-larva incubated at $29^{\circ} \mathrm{C}$; (d) DAPI; (e) merge. Scale bars: (a)-(c) $50 \mu \mathrm{m}$, (d)-(f) $100 \mu \mathrm{m}$.

in areas apparently lacking mitochondria or in organelleenriched areas. A further consequence of col4al mutation is the accumulation of nitrated and alkylated proteins in the mutants that localize to both normal and fused mitochondria.

This observation indicates a peroxynitrite-mediated nitrosative stress in col4al mutants that produce peroxynitrite at higher concentration [7]. We thus suggest that the elevated peroxynitrite level likely causes excess protein tyrosine nitration; however, this reaction does not deplete peroxynitrite in col4a1 mutants. Indeed, the still available peroxynitrite can initiate membrane damage by lipid peroxidation producing HNE, which in turn alkylates proteins by the mechanism of Michael addiction [8]. Direct association of alkylated proteins with the epithelial cell membrane of mutant Malpighian tubules supports this scenario. Furthermore, the mutationinduced stress directs the epithelia towards degeneration as demonstrated by the TUNEL-positivity of the nuclei.

The data presented here strongly suggest a central role for peroxynitrite in col4a1-associate defects. In wild-type animals and under physiological conditions, the nitrosoperoxycarbonate pathway is the preferential reaction of peroxynitrite, as the main decay products of nitrosoperoxycarbonate, nitrate anion, and carbon dioxide do not exert protein or membrane modification effects (Figure 5) [13]. In mutants, however, peroxynitrite is present above physiological concentrations, and it produces excess protein tyrosine nitration and forms
HNE leading to protein alkylation, lipid peroxidation, membrane damage, aberrant mitochondria, epithelial cell death, and Malpighian tubule dysfunction.

\section{Conclusions}

Drosophila with col4al mutation synthesize peroxynitrite as a part of their stress response above physiological concentrations. The excess peroxynitrite triggers heavy protein tyrosine nitration and protein alkylation adversely affecting protein function; it also initiates membrane lipid peroxidation and mitochondrial fusion. In control animals, these posttranslational protein modifications remain at physiological levels by utilizing the nitrosoperoxycarbonate pathway to neutralize peroxynitrite. We suggest that the posttranslational protein modifications detected in the col4al mutant Drosophila model are integral parts of col4al-associated pathology and represent pathomechanistic details that have not yet been addressed in human or mouse COL $4 A 1$ mutants.

\section{Conflicts of Interest}

The authors declare that there are no conflicts of interest regarding the publication of this paper. 


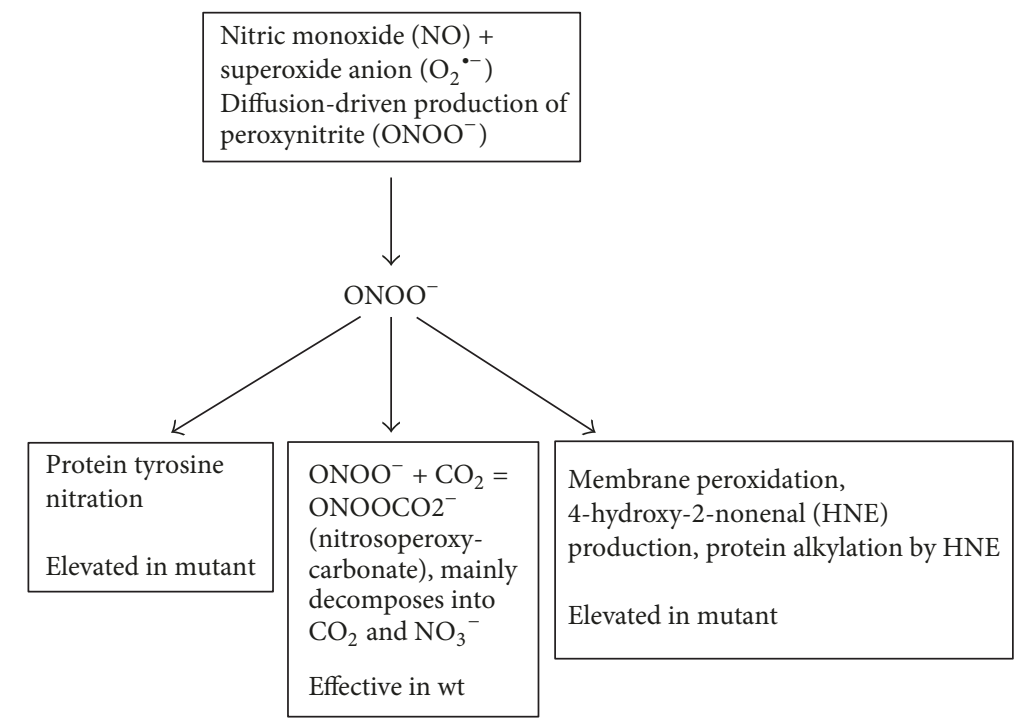

Figure 5: Schematic representation of peroxynitrite effects in wild-type flies shifting towards the neutralizing nitrosoperoxycarbonate pathway and in col4al mutants towards protein nitration and alkylation involving membrane peroxidation.

\section{Acknowledgments}

This research was supported by the Hungarian Scientific Research Fund OTKA, Contract no. NN 108283 to Mátyás Mink, and by the New National Excellence Program, Contract no. UNKP-17-3-I-SZTE-35 to András A. Kiss.

\section{References}

[1] A. Pozzi, P. D. Yurchenco, and R. V. Iozzo, "The nature and biology of basement membranes," Matrix Biology, vol. 57-58, pp. 1-11, 2017.

[2] M. Jeanne and D. B. Gould, "Genotype-phenotype correlations in pathology caused by collagen type IV alpha 1 and 2 mutations," Matrix Biology, vol. 57-58, pp. 29-44, 2017.

[3] D. S. Kuo, C. Labelle-Dumais, and D. B. Gould, "COL4A1 and COL4A2 mutations and disease: insights into pathogenic mechanisms and potential therapeutic targets," Human Molecular Genetics, vol. 21, no. 1, pp. R97-R110, 2012.

[4] Z. Chen, T. Migeon, M.-C. Verpont et al., "HANAC Syndrome Col4al Mutation Causes Neonate Glomerular Hyperpermeability and Adult Glomerulocystic Kidney Disease," Journal of the American Society of Nephrology: JASN, vol. 27, no. 4, pp. 10421054, 2016.

[5] I. Kelemen-Valkony, M. Kiss, J. Csiha et al., "Drosophila basement membrane collagen col4al mutations cause severe myopathy," Matrix Biology, vol. 31, no. 1, pp. 29-37, 2012.

[6] I. Kelemen-Valkony, M. Kiss, K. Csiszár, and M. Mink, Inherited Myopathies, Nova Publishers, New York, NY, USA, 2012.

[7] M. Kiss, A. A. Kiss, M. Radics et al., "Drosophila type IV collagen mutation associates with immune system activation and intestinal dysfunction," Matrix Biology, vol. 49, pp. 120-131, 2016.

[8] F. J. Schopfer, P. R. S. Baker, and B. A. Freeman, "NO-dependent protein nitration: A cell signaling event or an oxidative inflammatory response?" Trends in Biochemical Sciences, vol. 28, no. 12, pp. 646-654, 2003.
[9] A. Denicola and R. Radi, "Peroxynitrite and drug-dependent toxicity," Toxicology, vol. 208, no. 2, pp. 273-288, 2005.

[10] S. N. A. Hussain, G. Matar, E. Barreiro, M. Florian, M. Divangahi, and T. Vassilakopoulos, "Modifications of proteins by 4hydroxy-2-nonenal in the ventilatory muscles of rats," American Journal of Physiology-Lung Cellular and Molecular Physiology, vol. 290, no. 5, pp. L996-L1003, 2006.

[11] S. Dalleau, M. Baradat, F. Guéraud, and L. Huc, "Cell death and diseases related to oxidative stress: 4-hydroxynonenal (HNE) in the balance," Cell Death \& Differentiation, vol. 20, no. 12, pp. 1615-1630, 2013.

[12] G. Juric-Sekhar, K. Zarkovic, G. Waeg, A. Cipak, and N. Zarkovic, "Distribution of 4-hydroxynonenal-protein conjugates as a marker of lipid peroxidation and parameter of malignancy in astrocytic and ependymal tumors of the brain," TUMORI, vol. 95, no. 6, pp. 762-768, 2009.

[13] K. M. Nash, A. Rockenbauer, and F. A. Villamena, "Reactive nitrogen species reactivities with nitrones: Theoretical and experimental studies," Chemical Research in Toxicology, vol. 25, no. 8, pp. 1581-1597, 2012.

[14] J. A. T. Dow and M. F. Romero, "Drosophila provides rapid modeling of renal development, function, and disease," American Journal of Physiology-Renal Physiology, vol. 299, no. 6, pp. F1237-F1244, 2010.

[15] K. W. Beyenbach and P. L.-F. Liu, "Mechanism of fluid secretion common to aglomerular and glomerular kidneys," Kidney International, vol. 49, no. 6, pp. 1543-1548, 1996.

[16] A. A. Kiss, N. Popovics, G. Szabó, K. Csiszár, and M. Mink, "Altered stress fibers and integrin expression in the Malpighian epithelium of Drosophila type IV collagen mutants," Data in Brief, vol. 7, pp. 868-872, 2016.

[17] D. R. LaJeunesse, S. M. Buckner, J. Lake, C. Na, A. Pirt, and K. Fromson, "Three new Drosophila markers of intracellular membranes," BioTechniques, vol. 36, no. 5, pp. 784-790, 2004.

[18] R. Radi, "Nitric oxide, oxidants, and protein tyrosine nitration," Proceedings of the National Acadamy of Sciences of the United States of America, vol. 101, no. 12, pp. 4003-4008, 2004. 
[19] S. Chien, L. T. Reiter, E. Bier, and M. Gribskov, "Homophila: Human disease gene cognates in Drosophila," Nucleic Acids Research, vol. 30, no. 1, pp. 149-151, 2002.

[20] Y. Fu, J.-Y. Zhu, A. Richman et al., "A Drosophila model system to assess the function of human monogenic podocyte mutations that cause nephrotic syndrome," Human Molecular Genetics, vol. 26, no. 4, pp. 768-780, 2017.

[21] E. Plaisier, O. Gribouval, S. Alamowitch et al., "COL4A1 mutations and hereditary angiopathy, nephropathy, aneurysms, and muscle cramps," The New England Journal of Medicine, vol. 357, no. 26, pp. 2687-2695, 2007.

[22] Y. Weng, D. J. Dilworth, R. T. Libby, S. W. John, and D. B. Gould, "Mutant COL4A1 triggers oxidative stress in a genetic model of AMD," Matrix Biology, vol. 27, p. 39, 2008.

[23] R. J. Youle and A. M. van der Bliek, "Mitochondrial fission, fusion, and stress," Science, vol. 337, no. 6098, pp. 1062-1065, 2012. 


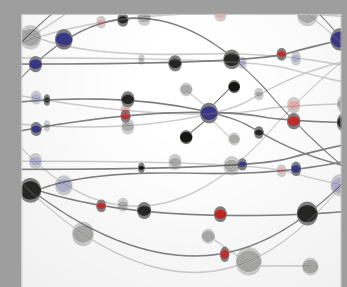

The Scientific World Journal
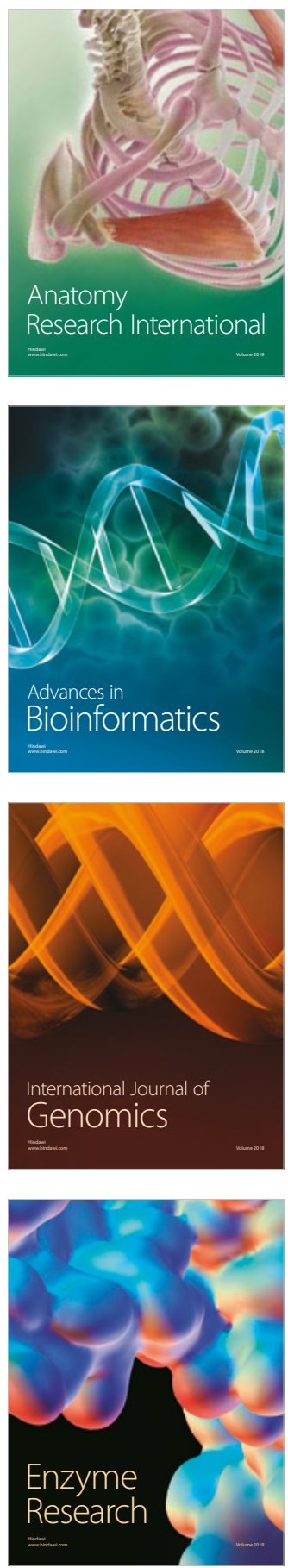
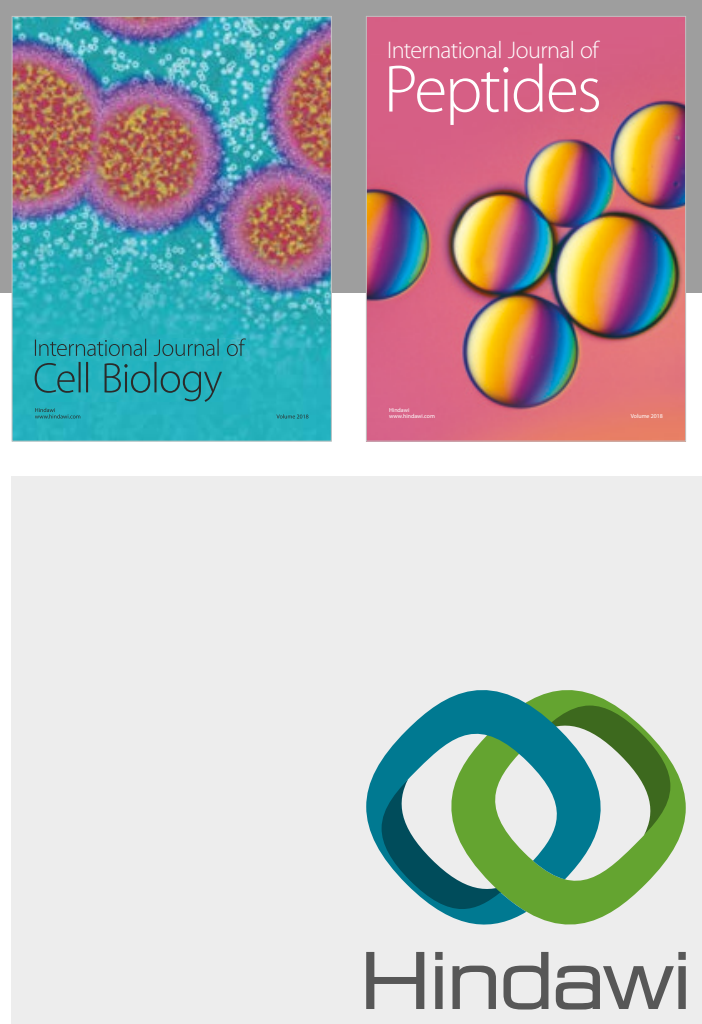

Submit your manuscripts at

www.hindawi.com
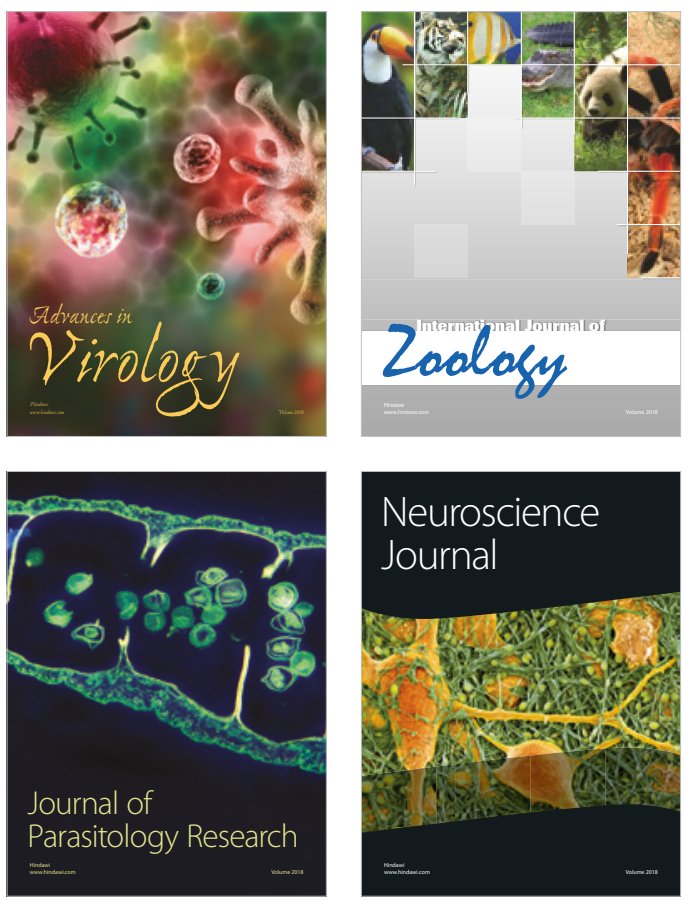
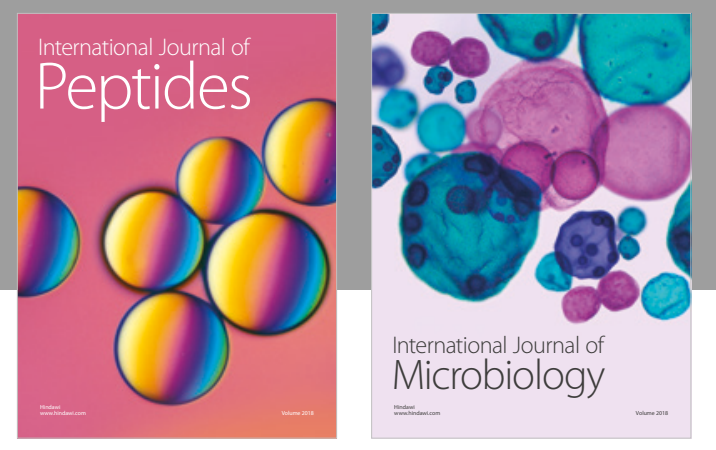

nternational Journal of Microbiology
Journal of
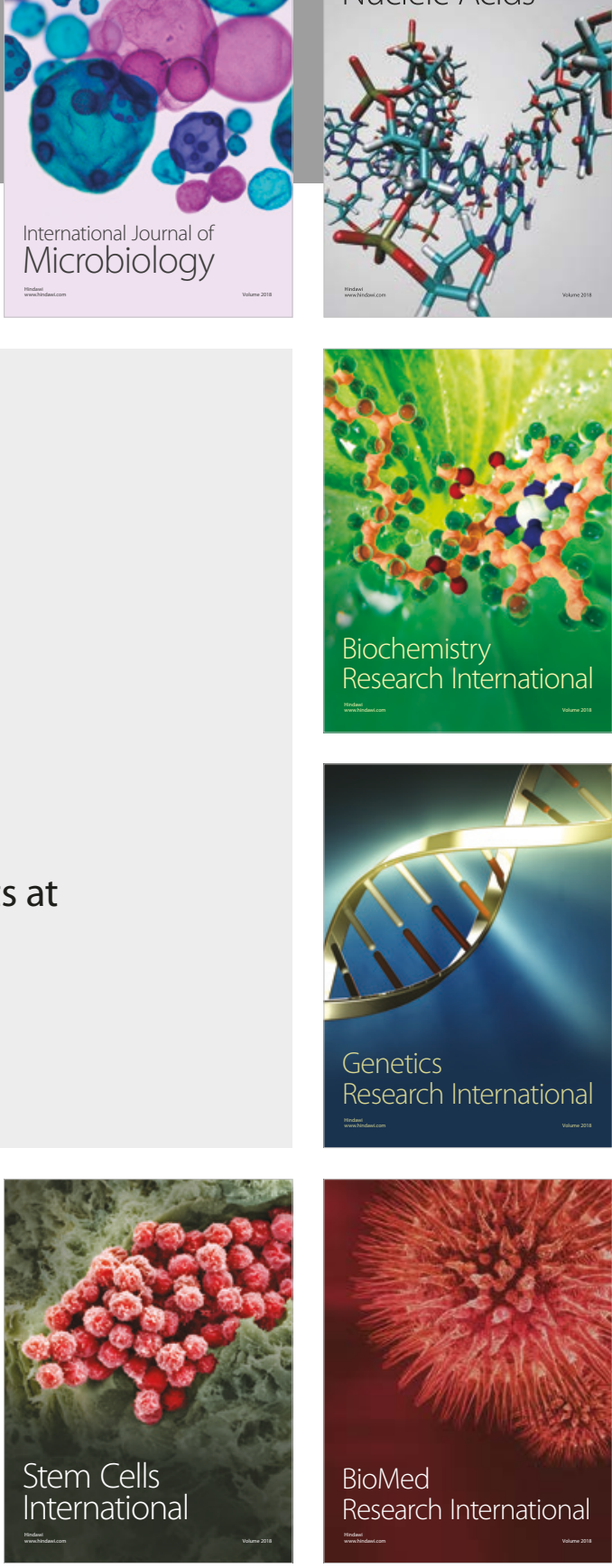
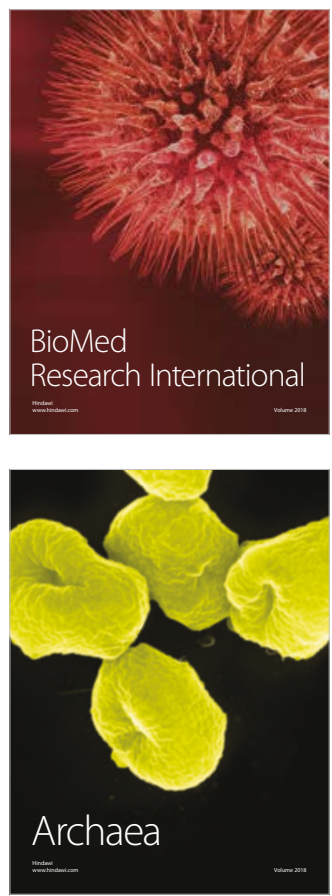


\section{Article V. \\ Novel Phenotypic Elements of Type IV Collagenopathy Revealed by the Drosophila Model}

$\underline{\text { András A. Kiss }}{ }^{1}$, Nikoletta Somlyai-Popovics ${ }^{1}$, Vilmos Tubak ${ }^{2}$, Zsolt Boldogkői ${ }^{1}$, Katalin Csiszár ${ }^{3}$ and Mátyás Mink ${ }^{1 *}$

Applied Sciences (2019)

https://doi.org/10.3390/app9102083

MTMT azonosító: 30686350

IF: $2.217(\mathbf{Q} 2)$ 
Article

\title{
Novel Phenotypic Elements of Type IV Collagenopathy Revealed by the Drosophila Model
}

\author{
András A. Kiss ${ }^{1}{ }^{\circledR}$, Nikoletta Somlyai-Popovics ${ }^{1}$, Vilmos Tubak ${ }^{2}{ }^{\circledR}$, Zsolt Boldogkói ${ }^{1}$, \\ Katalin Csiszár ${ }^{3}$ and Mátyás Mink ${ }^{1, * \mathbb{D}}$ \\ 1 Institute of Medical Biology, University of Szeged, H-6720 Szeged, Hungary; \\ kiss.andras.attila@med.u-szeged.hu (A.A.K.); somlyai-popovics.nikoletta@med.u-szeged.hu (N.S.-P.); \\ boldogkoi.zsolt@med.u-szeged.hu (Z.B.) \\ 2 Creative Laboratory Ltd., H-6726 Szeged, Hungary, tubak.vilmos@brc.mta.hu \\ 3 John A. Burns School of Medicine, University of Hawaii, Honolulu, HI 96822, USA; katalin@hawaiii.edu \\ * Correspondence: mink@bio.u-szeged.hu
}

Received: 5 April 2019; Accepted: 13 May 2019; Published: 21 May 2019

Featured Application: A great number of drugs can be screened with the aid of the high-throughput Drosophila system to combat type IV collagenopathy.

\begin{abstract}
Type IV collagen is proposed to be a key molecule in the evolvement of multicellular animals by forming the architectural unit basement membrane, a specialized form of the extracellular matrix. Functions of the basement membrane include guiding organ regeneration, tissue repair, modulation of cell differentiation, apical-basal polarity identification, cell migration and adhesion, regulation of growth factor signaling gradients, maintenance of tissue architecture and compartmentalization. Type IV collagenopathy is a devastating systemic disease affecting the circulatory, renal and visual systems and the skeletal muscles. It is observed in patients carrying mutations in the COL4A1 gene, which codes for the ubiquitous basement membrane component. Col4a1 mouse mutants display the human symptoms of type IV collagenopathy. We chose the Drosophila melanogaster model as we recorded dominant, temperature-sensitive mutations in the cognate col4a1 gene of the fruit fly and demonstrated phenotypic elements which have not yet been explored in humans or in mouse models. In this paper we show a transition of the Z-discs, normally bordering each sarcomere, to the level of M-discs significantly penetrant in the mutants, uneven distribution of fused mitochondria in the Malpighian tubules of the excretory organ and a loss of sarcomere structure in the visceral muscles in the gut of mutants. Our observations demonstrate the systemic nature of the coltal mutations in the fruit fly. However, these traits are elements of the type IV collagen-associated pathology and may provide insights into approaches that can alleviate symptoms of the disease.
\end{abstract}

Keywords: type IV collagen; mutation; multisystem disease; Drosophila model

\section{Introduction}

The highly differentiated form of the extracellular matrix is the planar-organized basement membrane (BM) found at the basal side of epithelial and endothelial cells in neural, vascular and adipose tissues, surrounding each individual striated muscle fiber.

Major proteins of BMs are type IV collagen, laminin, nidogen or entactin, perlecan and integrins [1]. Nidogens and perlecan are required perinatally but not at earlier phases of development [2]. The BM is anchored to the cell-surface integrin and to the dystroglycan receptors and provides a BM-cytoskeleton linkage [3]. The family of laminins consists of five alpha, four beta and three gamma chains in mammals. They harbor three pairs of type IV collagen genes, COL4A1 through to COL4A6, with each gene pair 
in head-to-head genomic organization [4-6]. Defects in BM components are often linked to human genetic diseases. An example of a laminin disorder is Lma2, merosin-deficient congenital muscular dystrophy (MDC1A and limb-girdle type) arising from lesions in the LAMA2 gene, it affects skeletal muscles and the neural system $[7,8]$. Mutations of the $L A M B 2$ gene cause Pierson syndrome, a form of focal segmental glomerulosclerosis [9].

Nearly 100 years ago, A.C. Alport reported about “hereditary familial congenital hemorrhagic nephritis" predominantly affecting men [10]. Type IV collagen genes were isolated many decades later, starting with COL4A1 [11], which was placed at the tip of the long arm of chromosome 13 [12]. Discovery of the rest of the five type IV collagen genes soon followed [13-17]. Association of the COL4A5 gene mutations was demonstrated in patients with X-chromosomal Alport syndrome $[18,19]$. Alport syndrome has a digenic inheritance. Only $80 \%$ of the patients carried mutations in the $\mathrm{X}$-chromosomal COL4A5 gene, whereas the rest had lesions in the autosomal COL4A3 locus at $2 q 36.3[20,21]$. The glomerular capillaries filter plasma through the glomerular basement membrane consisting of COL4A3, COL4A4 and COL4A5 heterotrimers. This isoform is absent from the glomeruli in patients with X-linked Alport syndrome and that harbor the fetal (COL4A1) ${ }_{2} \mathrm{COL} 4 \mathrm{~A} 2$ trimers, with increased susceptibility to proteolytic attack by collagenases and cathepsins $[22,23]$.

We identified dominant, temperature-sensitive mutations in the Drosophila type IV collagen gene coltal 1 exclusively. The col $4 a 1^{+/-}$heterozygotes are viable and fertile at $20^{\circ} \mathrm{C}$, the permissive temperature; however, they perish under the restrictive condition, $29^{\circ} \mathrm{C}$. The dominant-negative, antimorphic phenotype suggests the incorporation of mutant COL4A1 monomers into the planar-organized triple helices. The genome of the fruit fly consists of a pair of type IV collagen genes, col4a1 and col4a2 in a head-to-head orientation, similar to mammals [24]. The mutant phenotype is systemic, affecting the gut $[25,26]$, the excretory organ Malpighian tubules $[27,28]$ and the muscles manifested in muscular dystrophy [29]. The recessive phenotype of the col4a1 $\%$ homozygotes leads to lethality in the late embryonic to early larval phases at the permissive temperature [24]. Type IV collagen in Drosophila appears to fulfill a similar function as in mammals by providing BM stiffness and elasticity. Beyond conserved genomic organization, both genes and proteins share a high level of identity and similarity [24].

The clinical spectrum of COL4A1-associated defects is wide. Pedigrees of families in the Netherlands were identified as presenting cerebral porencephaly [30]. Mapping placed autosomal dominant type 1 porencephaly at the tip of chromosome arm 13q [31]. These families were assessed for mutations in the COL4A1 gene and missense Gly substitutions were demonstrated within the gly-X-Y repeats in the (COL4A1) ${ }_{2}$ COL4A2 triple helical domain [32]. Three families were diagnosed with autosomal dominant hereditary angiopathy with nephropathy, aneurysms and muscle cramps (HANAC) [33]. Sequencing revealed three close glycine mutations in the highly conserved exons 24 and 25 of the COL4A1 gene, affecting the integrin-binding site within the COL4A1 protein. Within the three families recapitulating the key features of HANAC, three different heterozygous missense mutations in the COL4A1 gene were demonstrated [34]. In a Spanish family with tortuosity of the retinal arteries, retinal hemorrhage was identified [35]. Interestingly, the lesion was not associated with muscle cramps, renal or brain anomalies, although the p.G510R mutation was previously detected in a French family with HANAC syndrome [34]. The different apparent expressivity of the associated trait was explained by environmental factors or genetic modifiers that may influence the phenotypic manifestation and the extent of organ involvement in COL4A1-related disease. In a study on the susceptibility to intracerebral hemorrhage, in two of 96 unrelated patients with adult-onset hemorrhagic stroke, the p.P352L substitution at the highly conserved Y-position of a gly-X-Y repeat and the p.R538G replacement were identified within the COL4A1 gene [36]. Heterozygosity for a missense mutation in the COL4A1 gene was identified in a boy with schizencephaly, renovascular hypertension, retinal arteriosclerosis and alveolar hemorrhage [37,38]. In 183 mostly pediatric patients with cerebral hemorrhage or porencephaly, 21 COL4A1 and three COL4A2 mutations were identified at a $40 \%$ sporadic mutation rate [39]. Diagnosis in some patients with COL4A1 mutations were consistent 
with Walker-Warburg Syndrome or muscle-eye-brain disease, a distinct form of congenital muscular dystrophy [40].

Mouse Col4a1 mutants recapitulate human disease. An allelic series of induced dominant mouse mutants was identified and showed ocular anterior segment defects or Axenfeld-Rieger anomaly, vacuolar cataracts, corneal opacities, iris defects, iris or corneal adhesions, buphthalmos, optic nerve cupping, retinal detachment and developed renal glomerulopathy [41]. Similar ophthalmic defects were noted in the Col4a1 splice site of mutant C57BL6/J mice. When C57BL6/J mice were crossed with 129/SvEvTac and CAST/Eij inbred strains, the F1 progeny were phenotypically almost indistinguishable from the wild-type. Genetic mapping identified a co-segregating dominant locus on chromosome 1, that likely contains the suppressor and modifier genes [42]. Col4a1 ${ }^{G 498 V / G 498 V}$ homozygous mice showed severe muscular dystrophy, including muscle mass decrease, fiber atrophy, centronuclear fibers, fibrosis, focal perivascular inflammation and intramuscular hemorrhaging [43]. Variants closer to the C-terminus tended to result in increased intracellular levels of the Col4a1 protein, whereas those closer to the $\mathrm{N}$-terminus tended to accumulate lower amounts of intracellular proteins with unchanged clinical severity [44]. The renal manifestation in mouse models of HANAC syndrome include albuminuria, hematuria, glomerular cysts and delays in glomerulogenesis and podocyte differentiation [45].

In col4a1 fly mutants, we recorded severe myopathy, reduced concentration of COL4A1 protein [24], chronic inflammation, intestinal dysfunction [25], detachment of the cells from the BM by electron microscopy [26], compromised excretory system [27], heavy membrane peroxidation in epithelial cells of the Malpighian tubules [28] and the onset of muscular dystrophy in all mutants [29]. Temperature-sensitive colta1 alleles in Drosophila were generated by ethyl-methanesulfonate mutagenesis, resulting in G to A transitions in all alleles, in turn leading to glycine substitutions by Glu, Asp and Ser [29]. The phenotypic changes appeared in adult flies following 14 days of incubation at $29^{\circ} \mathrm{C}$ and were not reversible, the flies did not recover at $20^{\circ} \mathrm{C}$ following a shift in heat. We established the complementation map of the col4a1-col4a2 locus with the aid of 34 mutants in all combinations in 561 independent crosses. The map was sharply divided into two non-overlapping parts with the dominant, temperature-sensitive alleles on one side, suggesting that only one of the two genes carries a temperature-sensitive mutation. Two mutant alleles of the same gene may restore the wild-type function in interallelic complementation [46]. We observed interallelic complementation among the temperature-sensitive col4a1 alleles. This genetic observation indirectly suggests that at least two COL4A1 chains interact in forming the trimeric protomer, allowing the homotrimer [COL4A1 $]_{3},[\mathrm{COL} 4 \mathrm{~A} 2]_{3}$ or heterotrimer [COL4A1 $]_{2} \mathrm{COL} 4 \mathrm{~A} 2$ composition of the triple helices [24]. In this paper, we present further phenotypic elements of the col4a1 mutations, including Z-disc shifting to the level of M-discs, aberrant mitochondria in the epithelial cells of the Malpighian tubules and loss of sarcomere structure in the visceral muscles, providing further data of a BM-linked systemic condition.

\section{Myopathic Defects in Drosophila col4a1 Mutants}

Under restrictive temperatures, the female heterozygotes become progressively more female-sterile [47]. We therefore chose the single-layer muscle fibers that encircle the common oviduct as a model. The system allowed the direct study of single muscle fibers and their sarcoplasmic membrane, without sectioning [24]. Muscle fibers in the col4a1 ${ }^{\mathrm{G} 552 D 2}$ mutant detached laterally from each other and a thickened collagen IV-associated label appeared to fill the gap. The basement membrane covering the myofibers appeared distorted, thinned or absent, demonstrating that the mutant COL4A1 protein induced severe oviductal myopathy $[24,26]$. Integrin deposition in muscle fibers was recorded by punctate staining at the level of Z-discs, overlapping with the signals provided by the Z-disc protein kettin and bordering each sarcomeric unit in immunohistochemical preparation [29]. High expressivity of the trait occurred at low penetrance, i.e., shifting Z-disc integrin-binding sites toward the middle of sarcomeres in col4a1 ${ }^{\mathrm{G} 552 D 1}$ mutants at the restrictive condition (Figure 1). The normal integrin-kettin staining still persisted at the level of the Z-discs and duplication of the Z-discs occurred at the level of M-discs (Figure 1, B4, B5, B6, white arrows). In some of the 
replications this phenomenon occurred at nearly full expressivity; integrin staining was shifted quantitatively up to the level of M-discs, into the middle of the anisotropic band (Figure 1, C1, C2, C3, white arrows).

\section{Oviductus}

\begin{tabular}{lll}
$20^{\circ} \mathbf{C}$ & Kettin-Integrin \\
\hline A1 & A2 & A3 \\
& & \\
B1 & & \\
& &
\end{tabular}

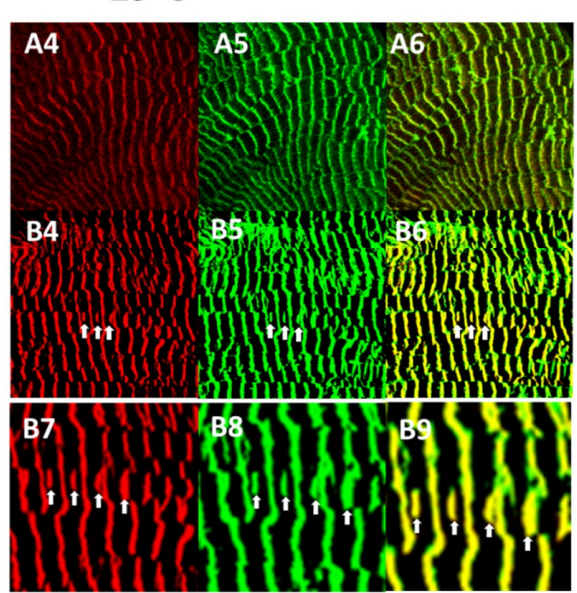

Wild-Type

col4a1 $1^{G 552 D 2}$

\section{$20^{\circ} \mathrm{C} \quad$ Actin-Integrin-DAPI}

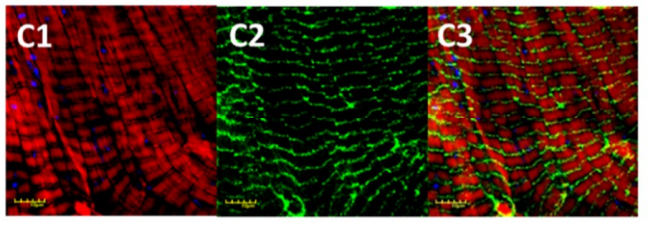

Wild-Type

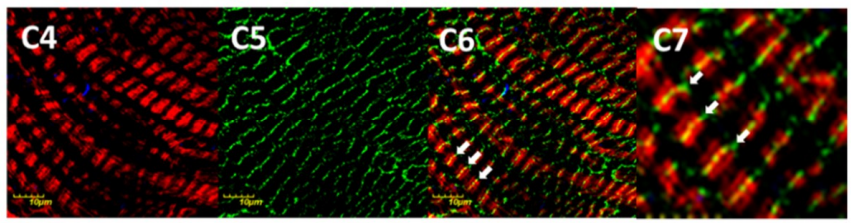

col4a1

Figure 1. Shifting of Z-discs into the middle of sarcomeres, to the level of M-discs in striated muscle fibers of oviducts. The scaffold protein kettin is deposited at the level of the Z-discs (A1, A4, B1, B4) and overlaps with the integrin label (A2, A5, B2, B5) providing yellow-orange complementary colors in the overlays (A3, A6, B3, B6). Note the irregular integrin expression in the mutant at $29^{\circ} \mathrm{C}$, zig-zag pattern and streaming of Z-discs (B5). Additional kettin-integrin expression appears in the middle of sarcomeres at the level of M-discs (white arrows, B4, B5, B6). Higher magnification of areas in B4, $\mathbf{B} 5$ and $\mathbf{B} 6$ are marked by arrows $(\mathbf{B 7}, \mathbf{B 8}, \mathbf{B 9})$. The shifting of the integrin expression toward the middle of the sarcomeres is nearly at full expressivity $(\mathbf{C 4}, \mathbf{C 5}, \mathbf{C 6}$, white arrows). The area marked by arrows in $\mathrm{C} 6$ are at higher magnification (C7). Integrin expression remains at the sides of sarcomeres in Z-discs in the wild-type control (C1, C2, C3). Bars, lower left: 10 micrometers. Experiments were carried out as described [24-26,28,29].

Next, we quantitatively analyzed the phenomenon of Z-disc shifting in the wild-type control (Oregon) and col4a1 ${ }^{G 52 D 1}$ mutants and we labeled the level of expressivity by the Z-disc marker, kettin (Figure 2). The expressivity of Z-disc shifting occurred at over $8 \%$ in the wild-type control and over $12 \%$ in col4a1 ${ }^{\text {G552D2 }}$ mutants (Figure 2). We obtained the results by counting 6796 kettin-staining structures within the single-layer striated muscle of the common oviduct. Statistical analysis with the aid of the Welch two-sample t-test revealed significance at a value of $p=0.003973$, indicating higher expressivity of the Z-disc shifting in the mutants (Figure 2). 


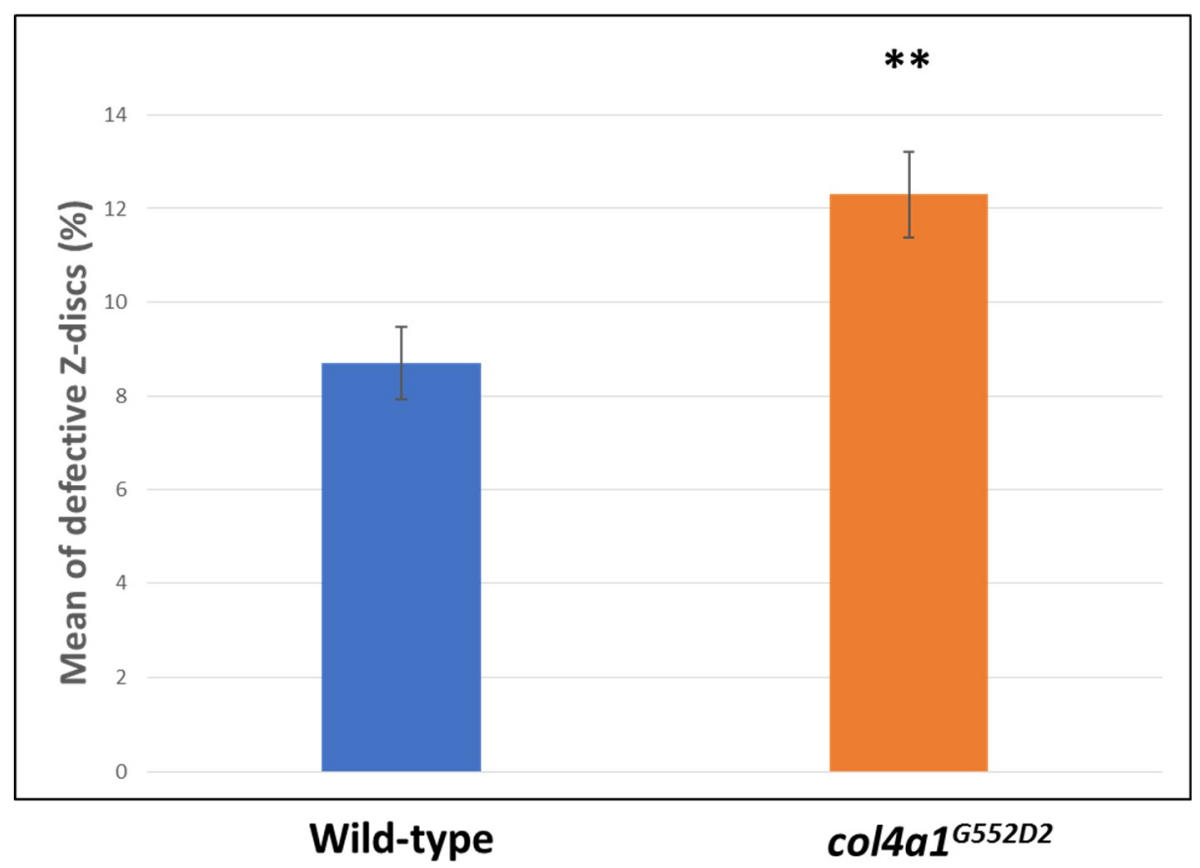

Figure 2. Elevated expressivity of Z-disc shifting in mutants. Statistically significant expressivity of Z-disc shifting in the mutants at $p=0.003973^{(* *)}$.

\section{Compromised Excretory System in the Mutants}

Excretion is accomplished by the kidneys in vertebrates and by the Malpighian tubules in insects. As part of the clinical spectrum amino acid substitutions in the ubiquitous human BM component, COL4A1 can cause nephropathy. Affected patients develop renal dysfunction and chronic kidney failure with or without hematuria. Mouse Col4a1 mutants present the same symptoms as humans. The Malpighian tubules are functionally similar to the mammalian kidneys and offer a versatile and tractable model [48]. The freely floating tubules are in continuous movement within the hemocoel, the blood-filled body cavity with open circulation. The mechanical load of periodic movements contributes to stress-induced cytoskeletal reorganization in col4a1 mutant animals. We therefore tested the effects of mechanical load in the col4a1 ${ }^{G 552 D 2}$ mutant and recorded the development of actin stress fibers and irregular, uneven actin staining in epithelial cells of the Malpighian tubules [27]. In a separate study, we noted mitochondrial fusion and co-localization of peroxynitrite-nitrated and 4-hydroxy-2-nonenal alkylated proteins with abnormal mitochondria [28]. Mitochondrial fusion in Malpighian epithelium is pronounced in the col4a $^{\mathrm{G} 552 D 1}$ mutant at the restrictive temperature (Figure 3), as a sign of the mutation-induced stress, whereas the level of protein nitration assessed qualitatively remain unchanged in the wild-type control and mutant strains under both the permissive and restrictive conditions (Figure 3). 


\section{Malpighian tubule}

$20^{\circ} \mathrm{C}$ Actin-Mitochondria-Nitrothyrosine

Wild-Type
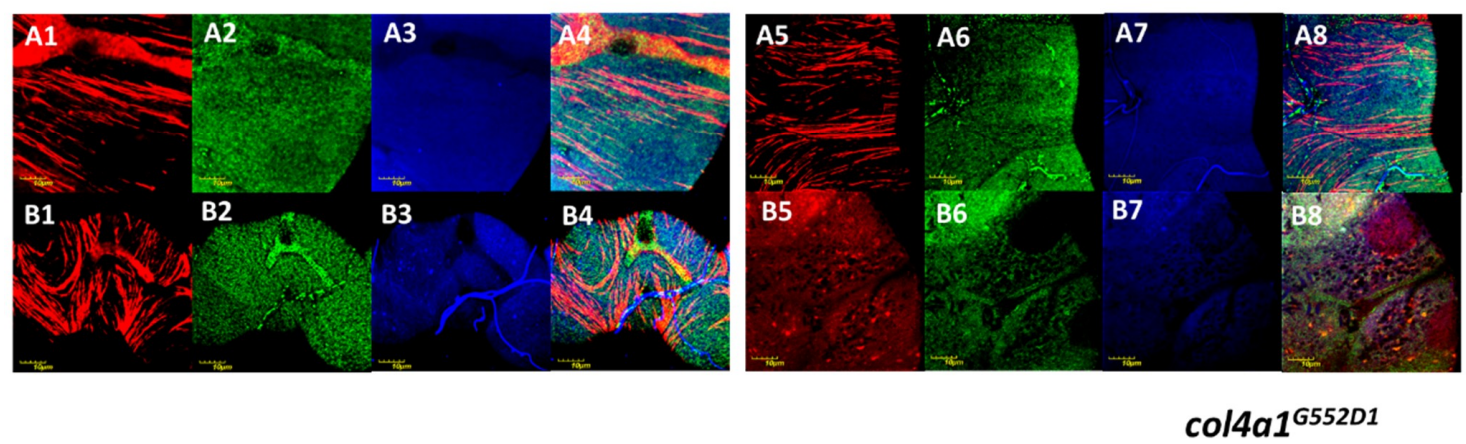

Figure 3. Compromised Malpighian tubules in mutants. Fusion and uneven distribution of mitochondria as a consequence of mutation-induced stress. Even distribution of mitochondria in wild-type control (A1, A2, A3, A4) and in the mutant under the permissive condition (B1, B2, B3, B4). The normal distribution of mitochondria remained at restrictive condition (A5, A6, A7, A8). Large aggregates of mitochondria appear in the mutant at $29^{\circ} \mathrm{C}(\mathbf{B} 5, \mathbf{B 6}, \mathbf{B 7}, \mathbf{B 8})$. Bars, lower left: 10 micrometers. Experiments were carried out as described [24-26,28,29].

\section{Intestinal Manifestation of col4a1 Mutations in Drosophila}

The skeletal and cardiac muscles appeared 700 million years ago in a common ancestor prior to the evolutionary divergence of vertebrates and arthropods. The smooth muscle of vertebrates is likely to have evolved later, independent of the skeletal and cardiac muscle types [49]. Accordingly, the visceral muscles of Drosophila are striated. We studied the larval midgut through ultrathin sectioning and electron microscopy and observed that both epithelial and visceral muscle cells were severely degenerated at the restrictive temperature in col4a1 ${ }^{G 552 D 2}$ mutant animals [26]. Both cell types detached from the distorted and thick BM, while epithelial cells accumulated lipid droplets, autophagic vacuoles and membrane ghosts. Membrane whorls practically filled the cell and the cytoplasm and muscle fibers were rarely observed. The space between the epithelial cell and the muscle cell was enlarged [26]. Functional damage of the alimentary tract in the col4a1 ${ }^{G 552 D 2}$ mutant was reflected by intestinal dysfunction, degeneration of the gut epithelial cells recorded by terminal deoxynucleotidyl transferase dUTP nick end labeling (TUNEL) in the nuclei and the abrupt onset of leakiness of the gut (SMURF phenotype), demonstrated by blue food dye leaking out from the gut [25]. Intestinal dysfunction, a motility-associated disorder including chronic intestinal pseudo-obstruction, is a direct, life-threatening and frequent complication of muscular dystrophies, requiring immediate surgical intervention [50]. Notwithstanding, complications arising from intestinal dysfunction reported in Drosophila col4a1 mutants in conjunction with COL4A1 mutations have not yet been noted in humans and mice. We suggest that intestinal dysfunction in the col4a1 ${ }^{G 52 D 2}$ mutant is a consequence of disrupted visceral muscle BM junction in the gut, without apparent sarcomere structure and amorphous, uneven actin deposition (Figure 4). 


\section{Gut}

\section{$20^{\circ} \mathrm{C} \quad$ Actin-COL4A1}

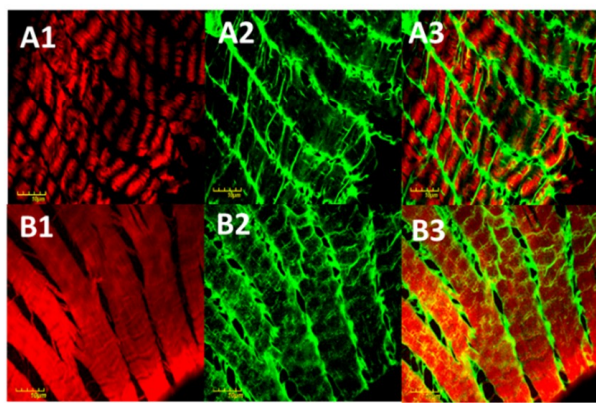

$29^{\circ} \mathrm{C}$

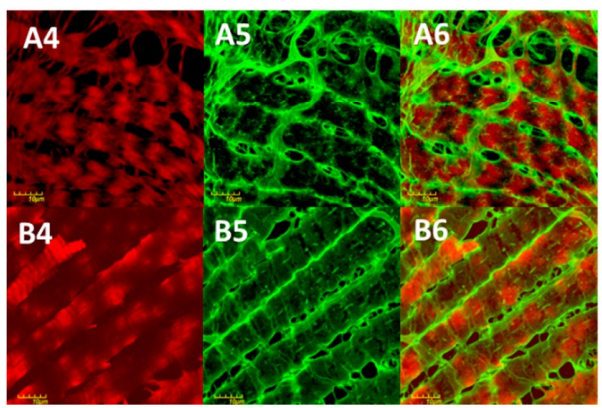

Wild-Type

col4a1 $1^{G 552 D 2}$

Figure 4. Loss of sarcomere structure in visceral muscle fibers. Regular sarcomere structure in the wild-type control at both temperatures (A1, A2, A3 and A4, A5, A6) and loss of sarcomeres in the mutant that deposits actin in an irregular fashion (B1, B2, B3 and B4, B5, B6). Bars, lower left: 10 micrometers. Experiments were carried out as described [24-26,28,29].

\section{Materials and Methods}

Wild-type Oregon flies and col $4 a 1$ mutant lines were maintained at $20^{\circ} \mathrm{C}$ and $29^{\circ} \mathrm{C}$ on yeast-cornmealsucrose-agar food, consisting of nipagin to prevent fungal infection. The mutant stocks were kept heterozygous over the recombination-preventing CyRoi balancer chromosome. Malpighian tubules, common oviducts and the gut were dissected under carbon dioxide anesthesia from adults that were grown at either the permissive or the restrictive temperature for 14 days. Dissected organs were fixed in a previously prepared $4 \%$ paraformaldehyde dissolved in phosphate buffered saline (PBS) solution for $10 \mathrm{~min}$, washed three times in PBS, permeabilized for $5 \mathrm{~min}$ in $0.1 \%$ Triton-X dissolved in PBS solution and washed three times in PBS. Blocking was achieved in a $\%$ bovine serum albumin dissolved in PBS solution for $1 \mathrm{~h}$, and washed three times in PBS.

Complementation tests were performed by ethyl-methanesulfonate-induced point mutants, P-element insertional mutants and X-ray-generated deletion (null) alleles [24]. The mutation sites in eight temperature-sensitive lines were sequenced. All mutations were $G$ to $A$ transitions and replaced glycine with glutamate, aspartate and serine. The $a$-30 allele carried the p.G233E amino acid substitution; the $b-9$ carried the p.G467E substitution. The isoallelic lines DTS-L2 and DTS-L3 carried the p.G552D1 and p.G552D2 substitutions, respectively. The second isoallelic pair DTS-L4 and DTS-L5 carried the p.G1205E1 and p.G1205E2 substitutions, respectively, and the DTS-L10 carried the p.G1043S substitution, whereas the $b-17$ carried the p.G1393E substitution. Importantly, the lesions covered the collagenous domain of the COL4A1 protein [29].

Nuclei in the dissected organs were counter-stained by $1 \mu \mathrm{g} / \mathrm{mL} 4^{\prime}$,6-diamino-2-phenylindol (DAPI) in $20 \mu \mathrm{L}$ PBS for 12 min in the dark. F-actin was stained by 1 unit Texas Red ${ }^{\text {TM}_{-}}$- Phalloidin (ThermoFisher) in $20 \mu \mathrm{L}$ PBS for $20 \mathrm{~min}$. Next, $1 \mu \mathrm{L}$ mouse monoclonal anti-3-Nitrothyrosine, anti-integrin alpha PS and anti-kettin antibodies (Abcam) were used in $20 \mu \mathrm{L}$ PBS for $1 \mathrm{~h}$. Mouse anti-COL4A1 antibodies were raised by Creative Laboratory Ltd., Szeged, Hungary. Primary mouse antibodies were visualized by $1 \mu \mathrm{L} \mathrm{F}\left(\mathrm{ab}^{\prime}\right)$ 2-Goat Anti-Mouse IgG $(\mathrm{H}+\mathrm{L})$ Cross Adsorbed Secondary Antibody conjugated with Alexa Fluor 488 (ThermoFisher) in $20 \mu \mathrm{L}$ PBS for $1 \mathrm{~h}$ and $1 \mu \mathrm{L}$ Goat Anti-Mouse IgG (H+L) Cross Adsorbed Secondary Antibody, Alexa Fluor 350, in $20 \mu \mathrm{L}$ PBS for $1 \mathrm{~h}$. Mitochondria were visualized by the mitochondrially targeted enhanced yellow fluorescent protein (mito-GFP) following appropriate crosses.

Photomicrographs were generated by confocal laser scanning fluorescence microscopy (Olympus Life Science Europa GmbH, Hamburg, Germany). The microscope configuration is described as follows. Objective lens: UPLSAPO 60× (oil, NA: 1.35); sampling speed: $8 \mu \mathrm{s} /$ pixel; line averaging: 2×; scanning 
mode: sequential unidirectional; excitation: $405 \mathrm{~nm}$ (DAPI), $543 \mathrm{~nm}$ (Texas Red) and $488 \mathrm{~nm}$ (Alexa Fluor 488); laser transmissivity: 7\% was used for DAPI, $42 \%$ for Alexa Fluor 488 and $52 \%$ for Texas Red.

\section{Conclusions}

The basement membrane is likely to be the primordial form of the extracellular matrix and type IV collagen the key molecule in the transition from uni- to multicellularity [51]. The visco-elasticity of the BM is provided by the type IV collagen network, with defects in this component compromising the network's elasticity and function. Type IV collagen proteins are highly conserved structurally and functionally during evolution, including the mammalian-Drosophila relationship [51]. The ubiquitous human and mammalian BM consists of (COL4A1) $)_{2} \mathrm{COL} 4 \mathrm{~A} 2$ heterotrimers and is distributed throughout the body. Glycine substitutions trigger systemic disease [1]. The genotype-phenotype relationship was established through 93 COL4A1 and 12 COL4A2 mutations. All of the lesions were dominant with nearly equal representation in males (48\%) and females (52\%). Inherited incidences were recorded at $47 \%$ and sporadic incidences at $53 \%$. As observed in other types of collagens, triple helical Gly substitutions were the most prevalent class of mutations (68 out of 93 in COL4A1) and frequent substitutions were charged amino acids; Arg (30), Glu (11) and Asp (10) [52].

Expressivity of the COL4A1 mutation-associated phenotypic elements often depends on genetic context in humans, mice and flies. In French families recapitulating key features of HANAC, different heterozygous missense mutations in the COL4A1 gene were demonstrated [34]. In a Spanish family, tortuosity of the retinal arteries and retinal hemorrhage were identified, but the lesion was not associated with muscle cramps, renal or brain anomalies, although the p.G510R mutation was previously detected in a French family with HANAC syndrome [35]. Crossing of the Col4a1 splice site mutant C57BL6/J mice with the 129/SvEvTac and CAST/EiJ inbred strains showed that the F1 progeny were phenotypically almost indistinguishable from the wild-type [42]. Similarly, in the isoallelic p.G552D1 and p.G552D2 mutants, the survival rates upon heat selection differed slightly. The line with the p.G552D1 lesion presented survival rates of $26.9 \%$ for pupae and $5.3 \%$ for adults, whereas the survival rates for the p.G552D2 line were $5.7 \%$ and $0.50 \%$, respectively [24,29].

Here, we provided data on the systemic phenotype of the col4a1 mutations and demonstrated the shifting of Z-discs, mitochondrial fusion and loss of sarcomeres in intestinal visceral muscle fibers. Genetic, genomic and proteomic data suggest that the fruit fly harbors all of the genetic elements of the $\mathrm{BM}$, although the number of genes coding for BM components is apparently reduced in comparison with mammals, increasing the genetic tractability of the fly mutants' defective genes coding for proteins of the BM that are structurally and functionally conserved during evolution, including type IV collagens [53]. The dominant col4a1 mutations therefore develop BM-specific phenotypes which are more severe than those observed in human and mouse models. Dominant temperature-sensitivity of the col4a1 alleles is a lethal condition, which cannot be maintained in mammals. Nevertheless, the col4a1-associated phenotypic elements provide numerous biomarkers that can be measured quantitatively during the screening of large numbers of drugs in high-throughput Drosophila screens, aimed at alleviating symptoms of type IV collagenopathy.

Author Contributions: Conceptualization and methodology, M.M.; validation, A.A.K., N.S-P., M.M.; formal analysis, A.A.K., N.S-P., M.M.; resources, Z.B., V.T.; writing-original draft preparation, M.M., K.C., Z.B.; supervision and project administration, M.M.

Funding: This research was funded by the Hungarian Scientific Research Fund OTKA, grant number NN 108283 to M.M. and by the UNKP-18-3 New National Excellence Program of the Ministry of Human Capacities, grant number UNKP-18-3-IV-SZTE-23 to A.A.K. and UNKP-18-3-IV-SZTE-43 to N.S.-P.

Conflicts of Interest: The authors declare no conflict of interest.

\section{References}

1. Pozzi, A.; Yurchenco, P.D.; Iozzo, R.V. The nature and biology of basement membranes. Matrix Biol. 2017, 57-58, 1-11. [CrossRef] 
2. Yurchenco, P.D.; Patton, B.L. Developmental and pathogenic mechanisms of basement membrane assembly. Curr. Pharm. Des. 2009, 15, 1277-1294. [CrossRef] [PubMed]

3. Sun, Z.; Guo, S.S.; Fässler, R. Integrin-mediated mechanotransduction. J. Cell Biol. 2016, 215, 445-456. [CrossRef] [PubMed]

4. Sado, Y.; Kagawa, M.; Naito, I.; Ueki, Y.; Seki, T.; Momota, R.; Oohashi, T.; Ninomiya, Y. Organization and expression of basement membrane collagen IV genes and their roles in human disorders. J. Biochem. 1998, 123, 767-776. [CrossRef]

5. Kashtan, C.E. Alport syndrome. An inherited disorder of renal, ocular, and cochlear basement membranes. Medicine (Baltimore) 1999, 78, 338-360. [CrossRef] [PubMed]

6. Miosge, N. The ultrastructural composition of basement membranes in vivo. Histol. Histopathol. 2001, 16, 1239-1248. [PubMed]

7. Jimenez-Mallebrera, C.; Brown, S.C.; Sewry, C.A.; Muntoni, F. Congenital muscular dystrophy: molecular and cellular aspects. Cell. Mol. Life Sci. 2005, 62, 809-823. [CrossRef]

8. Kanagawa, M.; Toda, T. The genetic and molecular basis of muscular dystrophy: roles of cell-matrix linkage in the pathogenesis. J. Hum. Genet. 2006, 51, 915-926. [CrossRef]

9. Matejas, V.; Hinkes, B.; Alkandari, F.; Al-Gazali, L.; Annexstad, E.; Aytac, M.B.; Barrow, M.; Bláhová, K.; Bockenhauer, D.; Cheong, H.I.; et al. Mutations in the human laminin beta2 (LAMB2) gene and the associated phenotypic spectrum. Hum. Mutat. 2010, 31, 992-1002. [CrossRef]

10. Alport, A.C. Hereditary Familial Congenital Haemorrhagic Nephritis. Br. Med. J. 1927, 1, 504-506. [CrossRef]

11. Pihlajaniemi, T.; Tryggvason, K.; Myers, J.C.; Kurkinen, M.; Lebo, R.; Cheung, M.C.; Prockop, D.J.; Boyd, C.D. cDNA clones coding for the pro-alpha1(IV) chain of human type IV procollagen reveal an unusual homology of amino acid sequences in two halves of the carboxyl-terminal domain. J. Biol. Chem. 1985, 260, 7681-7687.

12. Boyd, C.D.; Weliky, K.; Toth-Fejel, S.; Deak, S.B.; Christiano, A.M.; Mackenzie, J.W.; Sandell, L.J.; Tryggvason, K.; Magenis, E. The single copy gene coding for human alpha 1 (IV) procollagen is located at the terminal end of the long arm of chromosome 13. Hum. Genet. 1986, 74, 121-125. [CrossRef]

13. Brazel, D.; Pollner, R.; Oberbäumer, I.; Kühn, K. Human basement membrane collagen (type IV). The amino acid sequence of the alpha 2(IV) chain and its comparison with the alpha 1(IV) chain reveals deletions in the alpha 1(IV) chain. Eur. J. Biochem. 1988, 172, 35-42. [CrossRef]

14. Butkowski, R.J.; Langeveld, J.P.; Wieslander, J.; Hamilton, J.; Hudson, B.G. Localization of the Goodpasture epitope to a novel chain of basement membrane collagen. J. Biol. Chem. 1987, 262, 7874-7877.

15. Saus, J.; Wieslander, J.; Langeveld, J.P.; Quinones, S.; Hudson, B.G. Identification of the Goodpasture antigen as the alpha 3(IV) chain of collagen IV. J. Biol. Chem. 1988, 263, 13374-13380.

16. Hostikka, S.L.; Eddy, R.L.; Byers, M.G.; Höyhtyä, M.; Shows, T.B.; Tryggvason, K. Identification of a distinct type IV collagen alpha chain with restricted kidney distribution and assignment of its gene to the locus of $\mathrm{X}$ chromosome-linked Alport syndrome. Proc. Natl. Acad. Sci. USA 1990, 87, 1606-1610. [CrossRef]

17. Oohashi, T.; Sugimoto, M.; Mattei, M.G.; Ninomiya, Y. Identification of a new collagen IV chain, alpha 6(IV), by cDNA isolation and assignment of the gene to chromosome $\mathrm{Xq22,} \mathrm{which} \mathrm{is} \mathrm{the} \mathrm{same} \mathrm{locus} \mathrm{for} \mathrm{COL4A5.}$ J. Biol. Chem. 1994, 269, 7520-7526.

18. Antignac, C.; Zhou, J.; Sanak, M.; Cochat, P.; Roussel, B.; Deschênes, G.; Gros, F.; Knebelmann, B.; Hors-Cayla, M.C.; Tryggvason, K. Alport syndrome and diffuse leiomyomatosis: deletions in the $5^{\prime}$ end of the COL4A5 collagen gene. Kidney Int. 1992, 42, 1178-1183. [CrossRef]

19. Antignac, C.; Knebelmann, B.; Drouot, L.; Gros, F.; Deschênes, G.; Hors-Cayla, M.C.; Zhou, J.; Tryggvason, K.; Grünfeld, J.P.; Broyer, M. Deletions in the COL4A5 collagen gene in X-linked Alport syndrome. Characterization of the pathological transcripts in nonrenal cells and correlation with disease expression. J. Clin. Investig. 1994, 93, 1195-1207. [CrossRef]

20. Lemmink, H.H.; Mochizuki, T.; van den Heuvel, L.P.; Schröder, C.H.; Barrientos, A.; Monnens, L.A.; van Oost, B.A.; Brunner, H.G.; Reeders, S.T.; Smeets, H.J. Mutations in the type IV collagen alpha 3 (COL4A3) gene in autosomal recessive Alport syndrome. Hum. Mol. Genet. 1994, 3, 1269-1273. [CrossRef]

21. Lemmink, H.H.; Schröder, C.H.; Monnens, L.A.; Smeets, H.J. The clinical spectrum of type IV collagen mutations. Hum. Mutat. 1997, 9, 477-499. [CrossRef]

22. Kalluri, R.; Shield, C.F.; Todd, P.; Hudson, B.G.; Neilson, E.G. Isoform switching of type IV collagen is developmentally arrested in X-linked Alport syndrome leading to increased susceptibility of renal basement membranes to endoproteolysis. J. Clin. Investig. 1997, 99, 2470-2478. [CrossRef] 
23. Hudson, B.G.; Tryggvason, K.; Sundaramoorthy, M.; Neilson, E.G. Alport's Syndrome, Goodpasture's Syndrome, and Type IV Collagen. N. Engl. J. Med. 2003, 348, 2543-2556. [CrossRef]

24. Kelemen-Valkony, I.; Kiss, M.; Csiha, J.; Kiss, A.; Bircher, U.; Szidonya, J.; Maróy, P.; Juhász, G.; Komonyi, O.; Csiszár, K.; et al. Drosophila basement membrane collagen col4a1 mutations cause severe myopathy. Matrix Biol. 2012, 31, 29-37. [CrossRef]

25. Kiss, M.; Kiss, A.A.; Radics, M.; Popovics, N.; Hermesz, E.; Csiszár, K.; Mink, M. Drosophila type IV collagen mutation associates with immune system activation and intestinal dysfunction. Matrix Biol. 2016, 49, $120-131$. [CrossRef]

26. Kelemen-Valkony, I.; Kiss, M.; Csiszar, K.; Mink, M. Inherited Myopathies. In Myopathies: New Research; Washington, H.S., Jimenez, C.E.C., Eds.; Nova Science Publishers: Hauppauge, NY, USA, 2012; ISBN 9781622573721.

27. Kiss, A.A.; Popovics, N.; Szabó, G.; Csiszár, K.; Mink, M. Altered stress fibers and integrin expression in the Malpighian epithelium of Drosophila type IV collagen mutants. Data Brief 2016, 7, 868-872. [CrossRef]

28. Kiss, A.A.; Popovics, N.; Boldogkői, Z.; Csiszár, K.; Mink, M. 4-Hydroxy-2-nonenal Alkylated and Peroxynitrite Nitrated Proteins Localize to the Fused Mitochondria in Malpighian Epithelial Cells of Type IV Collagen Drosophila Mutants. BioMed Res. Int. 2018, 2018, 3502401. [CrossRef]

29. Kiss, A.A.; Popovics, N.; Marton, K.; Boldogkoi, Z.; Csiszar, K.; Mink, M. Type IV collagen is essential for proper function of integrin-mediated adhesion in Drosophila muscle fibers. bioRxiv 2018, 318337. [CrossRef]

30. Smit, L.M.; Barth, P.G.; Valk, J.; Njiokiktjien, C. Familial porencephalic white matter disease in two generations. Brain Dev. 1984, 6, 54-58. [CrossRef]

31. Aguglia, U.; Gambardella, A.; Breedveld, G.J.; Oliveri, R.L.; Le Piane, E.; Messina, D.; Quattrone, A.; Heutink, P. Suggestive evidence for linkage to chromosome 13qter for autosomal dominant type 1 porencephaly. Neurology 2004, 62, 1613-1615. [CrossRef]

32. Gould, D.B.; Phalan, F.C.; Breedveld, G.J.; van Mil, S.E.; Smith, R.S.; Schimenti, J.C.; Aguglia, U.; van der Knaap, M.S.; Heutink, P.; John, S.W.M. Mutations in Col4a1 Cause Perinatal Cerebral Hemorrhage and Porencephaly. Science (80-.) 2005, 308, 1167-1171. [CrossRef]

33. Plaisier, E.; Gribouval, O.; Alamowitch, S.; Mougenot, B.; Prost, C.; Verpont, M.C.; Marro, B.; Desmettre, T.; Cohen, S.Y.; Roullet, E.; et al. COL4A1 Mutations and Hereditary Angiopathy, Nephropathy, Aneurysms, and Muscle Cramps. N. Engl. J. Med. 2007, 357, 2687-2695. [CrossRef]

34. Plaisier, E.; Chen, Z.; Gekeler, F.; Benhassine, S.; Dahan, K.; Marro, B.; Alamowitch, S.; Paques, M.; Ronco, P. Novel COL4A1 mutations associated with HANAC syndrome: a role for the triple helical CB3[IV] domain. Am. J. Med. Genet. A 2010, 152A, 2550-2555. [CrossRef]

35. Zenteno, J.C.; Crespí, J.; Buentello-Volante, B.; Buil, J.A.; Bassaganyas, F.; Vela-Segarra, J.I.; Diaz-Cascajosa, J.; Marieges, M.T. Next generation sequencing uncovers a missense mutation in COL4A1 as the cause of familial retinal arteriolar tortuosity. Graefe's Arch. Clin. Exp. Ophthalmol. 2014, 252, 1789-1794. [CrossRef]

36. Weng, Y.-C.; Sonni, A.; Labelle-Dumais, C.; de Leau, M.; Kauffman, W.B.; Jeanne, M.; Biffi, A.; Greenberg, S.M.; Rosand, J.; Gould, D.B. COL4A1 mutations in patients with sporadic late-onset intracerebral hemorrhage. Ann. Neurol. 2012, 71, 470-477. [CrossRef]

37. Yoneda, Y.; Haginoya, K.; Kato, M.; Osaka, H.; Yokochi, K.; Arai, H.; Kakita, A.; Yamamoto, T.; Otsuki, Y.; Shimizu, S.; et al. Phenotypic Spectrum of COL4A1 Mutations: Porencephaly to Schizencephaly. Ann. Neurol. 2013, 73, 48-57. [CrossRef]

38. Abe, Y.; Matsuduka, A.; Okanari, K.; Miyahara, H.; Kato, M.; Miyatake, S.; Saitsu, H.; Matsumoto, N.; Tomoki, M.; Ihara, K. A severe pulmonary complication in a patient with COL4A1-related disorder: A case report. Eur. J. Med. Genet. 2017, 60, 169-171. [CrossRef]

39. Meuwissen, M.E.C.; Halley, D.J.J.; Smit, L.S.; Lequin, M.H.; Cobben, J.M.; de Coo, R.; van Harssel, J.; Sallevelt, S.; Woldringh, G.; van der Knaap, M.S.; et al. The expanding phenotype of COL4A1 and COL4A2 mutations: clinical data on 13 newly identified families and a review of the literature. Genet. Med. 2015, 17, 843-853. [CrossRef]

40. Labelle-Dumais, C.; Dilworth, D.J.; Harrington, E.P.; de Leau, M.; Lyons, D.; Kabaeva, Z.; Manzini, M.C.; Dobyns, W.B.; Walsh, C.A.; Michele, D.E.; et al. COL4A1 Mutations Cause Ocular Dysgenesis, Neuronal Localization Defects, and Myopathy in Mice and Walker-Warburg Syndrome in Humans. PLoS Genet. 2011, 7, e1002062. [CrossRef] 
41. Van Agtmael, T.; Schlötzer-Schrehardt, U.; McKie, L.; Brownstein, D.G.; Lee, A.W.; Cross, S.H.; Sado, Y.; Mullins, J.J.; Pöschl, E.; Jackson, I.J. Dominant mutations of Col4a1 result in basement membrane defects which lead to anterior segment dysgenesis and glomerulopathy. Hum. Mol. Genet. 2005, 14, 3161-3168. [CrossRef]

42. Gould, D.B.; Marchant, J.K.; Savinova, O.V.; Smith, R.S.; John, S.W.M. Col4a1 mutation causes endoplasmic reticulum stress and genetically modifiable ocular dysgenesis. Hum. Mol. Genet. 2007, 16, 798-807. [CrossRef]

43. Guiraud, S.; Migeon, T.; Ferry, A.; Chen, Z.; Ouchelouche, S.; Verpont, M.-C.; Sado, Y.; Allamand, V.; Ronco, P.; Plaisier, E. HANAC Col4a1 Mutation in Mice Leads to Skeletal Muscle Alterations due to a Primary Vascular Defect. Am. J. Pathol. 2017, 187, 505-516. [CrossRef]

44. Kuo, D.S.; Labelle-Dumais, C.; Mao, M.; Jeanne, M.; Kauffman, W.B.; Allen, J.; Favor, J.; Gould, D.B. Allelic heterogeneity contributes to variability in ocular dysgenesis, myopathy and brain malformations caused by Col4a1 and Col4a2 mutations. Hum. Mol. Genet. 2014, 23, 1709-1722. [CrossRef]

45. Chen, Z.; Migeon, T.; Verpont, M.-C.; Zaidan, M.; Sado, Y.; Kerjaschki, D.; Ronco, P.; Plaisier, E. HANAC Syndrome Col4a1 Mutation Causes Neonate Glomerular Hyperpermeability and Adult Glomerulocystic Kidney Disease. J. Am. Soc. Nephrol. 2016, 27, 1042-1054. [CrossRef]

46. Clifford, R.; Schüpbach, T. Molecular analysis of the Drosophila EGF receptor homolog reveals that several genetically defined classes of alleles cluster in subdomains of the receptor protein. Genetics 1994, 137, 531-550.

47. Suzuki, D.T.; Procunier, D. Temperature-sensitive mutations in Drosophila melanogaster. 3. Dominant lethals and semilethals on chromosome 2. Proc. Natl. Acad. Sci. USA 1969, 62, 369-376. [CrossRef]

48. Dow, J.A.T.; Romero, M.F. Drosophila provides rapid modeling of renal development, function, and disease. Am. J. Physiol. 2010, 299, F1237-F1244. [CrossRef]

49. OOta, S.; Saitou, N. Phylogenetic relationship of muscle tissues deduced from superimposition of gene trees. Mol. Biol. Evol. 1999, 16, 856-867. [CrossRef]

50. Connor, F.L.; Di Lorenzo, C. Chronic Intestinal Pseudo-obstruction: Assessment and Management. Gastroenterology 2006, 130, S29-S36. [CrossRef]

51. Fidler, A.L.; Darris, C.E.; Chetyrkin, S.V.; Pedchenko, V.K.; Boudko, S.P.; Brown, K.L.; Gray Jerome, W.; Hudson, J.K.; Rokas, A.; Hudson, B.G. Collagen IV and basement membrane at the evolutionary dawn of metazoan tissues. eLife 2017, 6, e24176. [CrossRef]

52. Jeanne, M.; Gould, D.B. Genotype-phenotype correlations in pathology caused by collagen type IV alpha 1 and 2 mutations. Matrix Biol. 2016. [CrossRef]

53. Ramos-Lewis, W.; Page-McCaw, A. Basement membrane mechanics shape development: Lessons from the fly. Matrix Biol. 2018. [CrossRef] 


\section{Article VI.}

Type IV Collagen Is Essential for Proper Function of Integrin-Mediated Adhesion in Drosophila Muscle Fibers

András A. Kisss ${ }^{1}$, Nikoletta Somlyai-Popovics ${ }^{1}$, Márton Kiss ${ }^{1}$, Zsolt

Boldogkői ${ }^{1}$, Katalin Csiszár $^{2}$ and Mátyás Mink ${ }^{1}$

International Journal of Molecular Sciences (2019)

https://doi.org/10.3390/ijms20205124

MTMT azonosító: 30881918

IF: $4.183(Q 2)$ 


\title{
Type IV Collagen Is Essential for Proper Function of Integrin-Mediated Adhesion in Drosophila Muscle Fibers
}

\author{
András A. Kiss ${ }^{1}{ }^{\circledR}$, Nikoletta Somlyai-Popovics ${ }^{1}$, Márton Kiss ${ }^{1}$, Zsolt Boldogkói ${ }^{1}$, Katalin Csiszár ${ }^{2}$ \\ and Mátyás Mink $1, * \mathbb{D}$ \\ 1 Institute of Medical Biology, University of Szeged, Somogyi B. u. 4, H-6720 Szeged, Hungary; \\ kiss.andras.attila@med.u-szeged.hu (A.A.K.); somlyai-popovics.nikoletta@med.u-szeged.hu (N.S.-P.); \\ mxk044@shsu.edu (M.K.); boldogkoi.zsolt@med.u-szeged.hu (Z.B.) \\ 2 John A. Burns School of Medicine, University of Hawaii, 1960 East West Road, Honolulu, HI 96822, USA; \\ katalin@hawaiii.edu \\ * Correspondence: mink@bio.u-szeged.hu
}

Received: 29 August 2019; Accepted: 13 October 2019; Published: 16 October 2019

\begin{abstract}
Congenital muscular dystrophy (CMD), a subgroup of myopathies is a genetically and clinically heterogeneous group of inherited muscle disorders and is characterized by progressive muscle weakness, fiber size variability, fibrosis, clustered necrotic fibers, and central myonuclei present in regenerating muscle. Type IV collagen (COL4A1) mutations have recently been identified in patients with intracerebral, vascular, renal, ophthalmologic pathologies and congenital muscular dystrophy, consistent with diagnoses of Walker-Warburg Syndrome or Muscle-Eye-Brain disease. Morphological characteristics of muscular dystrophy have also been demonstrated Col4a1 mutant mice. Yet, several aspects of the pathomechanism of COL4A1-associated muscle defects remained largely uncharacterized. Based on the results of genetic, histological, molecular, and biochemical analyses in an allelic series of Drosophila col4a1 mutants, we provide evidence that col4a1 mutations arise by transitions in glycine triplets, associate with severely compromised muscle fibers within the single-layer striated muscle of the common oviduct, characterized by loss of sarcomere structure, disintegration and streaming of Z-discs, indicating an essential role for the COL4A1 protein. Features of altered cytoskeletal phenotype include actin bundles traversing over sarcomere units, amorphous actin aggregates, atrophy, and aberrant fiber size. The mutant COL4A1-associated defects appear to recapitulate integrin-mediated adhesion phenotypes observed in RNA-inhibitory Drosophila. Our results provide insight into the mechanistic details of COL4A1-associated muscle disorders and suggest a role for integrin-collagen interaction in the maintenance of sarcomeres.
\end{abstract}

Keywords: Type IV collagen; basement membrane; mutation; myopathy; dystrophy

\section{Introduction}

Basement membranes (BMs) are 80-100 nm thick, sheet-like extracellular matrices underlying epithelial and endothelial cells in muscular, neural, vascular and adipose tissues. BMs contain major and minor proteins, including type IV collagen, laminin, nidogen/entactin, perlecan, and integrins [1]. Integrity of the BM is a prerequisite for skeletal muscle stability. Research revealed that several muscular dystrophy types may develop as the result of the loss of cell-BM anchorage [2]. Causative gene mutations were reported in the laminin-A2 (LAMA2) gene [3,4]. Mutations in the collagen VI genes were linked to Ullrich CMD, to the milder Bethlem myopathy and to autosomal recessive myosclerosis myopathy [5], while integrin A7 (ITGA7) and A5 (ITGA5) mutations were shown to be associated to a rare form of CMD [6-8]. 
Mammals harbor three pairs of head-to-head oriented type IV collagen genes, whereas Drosophila has one, the col4a1 and col4a2 loci in the same genomic organization [9]. Mutations in the COL4A3, A4 and $A 5$ genes associate with Alport Syndrome, [10,11]. Deletions within the COL4A5 and COL4A6 genes are also reported to cause diffuse leiomyomatosis [12]. Heterotrimers with [COL4A1] ${ }_{2}$ COL4A2 composition constitute stochiometrically the most abundant mammalian basement membranes. Col4a1 or Col4a2 mouse mutants develop complex, systemic phenotypes affecting the central nervous, ocular, renal, pulmonary, vascular, reproductive and muscular systems [13-15], as in humans [16]. Severe muscular phenotypes were reported in patients with certain COL4A1 mutations as part of a multi-system disorder referred to as hereditary angiopathy with nephropathy, aneurysms, and muscle cramps (HANAC) $[17,18]$. Some patients with COL4A1 mutations were also diagnosed with Walker-Warburg Syndrome or Muscle-Eye-Brain disease, a distinct form of CMD [19]. Col4a1 ${ }^{\text {G498V/G498V homozygous }}$ mice are severely affected by muscular dystrophy including muscle mass decrease, fiber atrophy, centronuclear fibers, fibrosis, focal perivascular inflammation, and intramuscular hemorrhages [20].

In HANAC Syndrome, mutations proved to affect multiple putative integrin binding sites within the COL4A1 protein [21,22]. Proper integrin concentration/function was shown to be required for maintenance of the sarcomere structure [23]. Drosophila integrin null mutants loose sarcomeres completely [24], where the ubiquitous integrin dimer is composed of one of the alpha PS subunits combined with the beta PS protein [25]. Conditional RNAi knockdown of talin, alpha-actinin, integrin-linked kinase, alpha PS2 and beta PS integrins revealed a spectrum of phenotypes affecting Z-disc proteins that were dislocated and deposited across the sarcomere, and Z-disc streaming characteristic of myopathic/dystrophic conditions [26].

We have identified an allelic series of conditional, temperature-sensitive col4a1 mutations in Drosophila. The col4a1 $1^{-/}$homozygotes are embryonic lethal while colta1 $1^{+/-}$heterozygotes are viable and fertile at permissive temperature of $20^{\circ} \mathrm{C}$, but perish at restrictive condition of $29^{\circ} \mathrm{C}$. In these mutants we have demonstrated severe myopathy [9], irregular and thickened BM, detachment of the gut epithelial and visceral muscle cells from the BM [27], intestinal dysfunction, overexpression of antimicrobial peptides, excess synthesis of hydrogen peroxide and peroxynitrite [28]. In epithelial cells of Malpighian tubules we demonstrated fused mitochondria, membrane peroxidation [29], actin stress fibers and irregular integrin expression [30]. Our results indicated that muscular dystrophy may also be present in col4a1 mutant Drosophila [31].

In order to characterize muscle phenotype in the col4a1 allelic mutant series we have determined the mutation sites, in immunohistochemistry experiments focused on the striated oviduct muscle we noted aberrant sarcomeres, altered integrin expression and localization, Z-disc disorganization and streaming, fiber size disproportion and atrophy. Results collectively indicate that in mutants dystrophic muscle phenotype appears to originate from compromised integrin interactions with aberrant COL4A1, and supports a role for type IV collagen as part of integrin-mediated muscle cell adhesion.

\section{Results and Discussion}

\subsection{Characterization of col4a1 Mutation Sites}

We analyzed the DNA sequence of PCR products of the col4a1 gene using genomic DNA isolated from our series of $c o l 4 a 1^{+/-}$heterozygotes. Consistent with the ethyl-methane-sulfonate (EMS) mutagenesis used to generate these mutants [9], by which the product, O-6-ethylguanosine, mispairs with $\mathrm{T}$ in the next round of replication, causing a G/C to A/T transition, we identified transition in all mutant loci (Supplementary Figure S1). Resulting from these transitions glycine substitutions by aspartic acid, glutamic acid or serine were identified in the mutants; hereafter we refer to the mutations as displayed in Table 1. In our prior study we reported five mutations in the col4a1 gene using the DTS-L3 allele; the G552D and the A1081T amino acid substitutions arose by transitions, similarly to the G to A transition within the 3' UTR region of the gene. The K1125N, G1198A amino acid substitutions are results of transversions [9]. The A1081T, the $3^{\prime}$ UTR transitions and the K1125N, 
G1198A transversions occurred in all sequenced mutants. We therefore concluded that these mutations are carried by the balancer chromosome, CyRoi. These balancer chromosome mutations do not contribute to the phenotype, given the most robust phenotypical feature of the mutants, the dominant temperature sensitivity is not influenced by exchange of the CyRoi chromosome into the chromosome carrying the col4a2::GFP transgene [9].

Table 1. The mutation sites, former and present designation of the mutant loci.

\begin{tabular}{|c|c|c|c|c|}
\hline $\begin{array}{c}\text { Former } \\
\text { Designation }\end{array}$ & $a-30$ & $b-9$ & DTS-L2 & DTS-L3 \\
\hline \multirow{3}{*}{$\begin{array}{l}\text { Present designation } \\
\text { Mutation site }\end{array}$} & $\operatorname{col}_{4 a 1}{ }^{\mathrm{G} 233 E}$ & $\operatorname{col}_{4 a 1}{ }^{\mathrm{G} 467 E}$ & col4a1 $^{G 552 D 1}$ & $\operatorname{col}_{4 a 1^{G 552 D 2}}$ \\
\hline & p.G233E & p.G467E & p.G552D1 & p.G225D2 \\
\hline & GGA->GAA & GGA->GAA & GGC->GAC & GGC->GAC \\
\hline Former designation & DTS-L4 & DTS-L5 & DTS-L10 & $b-17$ \\
\hline \multirow{3}{*}{$\begin{array}{l}\text { Present designation } \\
\text { Mutation site }\end{array}$} & col4a1 $^{\text {G1025E1 }}$ & col4a1 ${ }^{\text {G1025E2 }}$ & col4a1 $^{\text {G1043S }}$ & col4a1 $^{\text {G1393E }}$ \\
\hline & p.G1205E1 & p.G1025E2 & p.G1043S & p.G1393E \\
\hline & GGA->GAA & GGA->GAA & GGG->GAG & GGA->GAA \\
\hline
\end{tabular}

Two pairs of alleles were found to carry the same mutation. The DTS-L2 and DTS-L3 lines harbor the same G552D substitution and we refer to these as col4a1 ${ }^{G 552 D 1}$ and col4a1 ${ }^{\text {G552D2 }}$. Similarly, the DTS-L4 and DTS-L5 lines both carry the G1025E substitution and were designated as col4a1 ${ }^{\text {G1025E1 }}$ and col4a1 ${ }^{\text {G1025E2 }}$ alleles. These data confirmed our previous genetic results, for example: The col4a1 ${ }^{\mathrm{G} 552 D 1}$ and col4a1 ${ }^{\mathrm{G5} 52 D 2}$ lines did not complement each other, but complemented the other variants by interallelic complementation [9]. The distribution of the mutant sites at protein level is demonstrated in Figure 1.

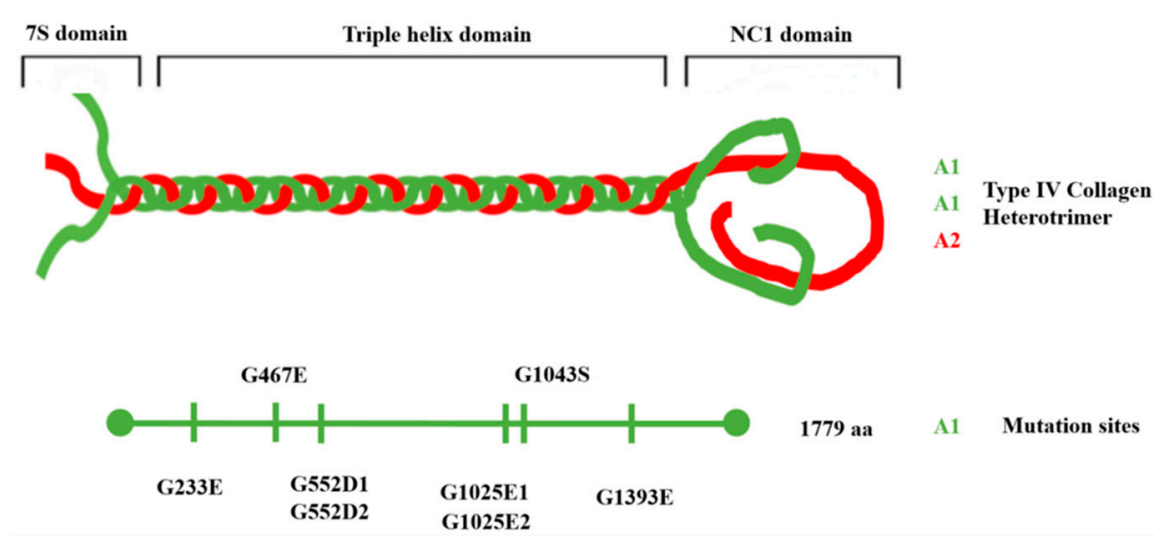

Figure 1. Triple-helical model of type IV collagen trimer with [COL4A1]2COL4A1 composition and distribution of the mutation sites on the COL4A1 protein.

The series of mutations proved to cover the collagenous region of the colta1 gene that corresponds to amino acid 233 up to 1393 within the $170 \mathrm{kDa}$ COL4A1 protein. The col4a1 ${ }^{\mathrm{G} 233 E}$ allele is within the peptide GFPG/EEKGERGD (the G to E substitution in bold), a putative integrin binding site in the COL4A1 protein [21]. The mutation sites in the col4a1 ${ }^{G 552 D 1}$ and col4a1 ${ }^{G 52 D 2}$ lines localize in the immediate proximity of the peptide GLPGEKGLRGD, that resembles the integrin binding site in the COL4A2 protein in the triple helical model made up of [COL4A1 $]_{2}$ COL4A2 protomers in Drosophila, as proposed by us [9] and presented graphically in Figure 1. 


\subsection{Loss of Sarcomere Structure in col4a1 Mutants}

The single-layer striated muscle fibers circumventing the common oviduct were analyzed in all mutants by confocal fluorescence microscopy. Normally, these muscle fibers present regular sarcomere structure as demonstrated in Figure 2.

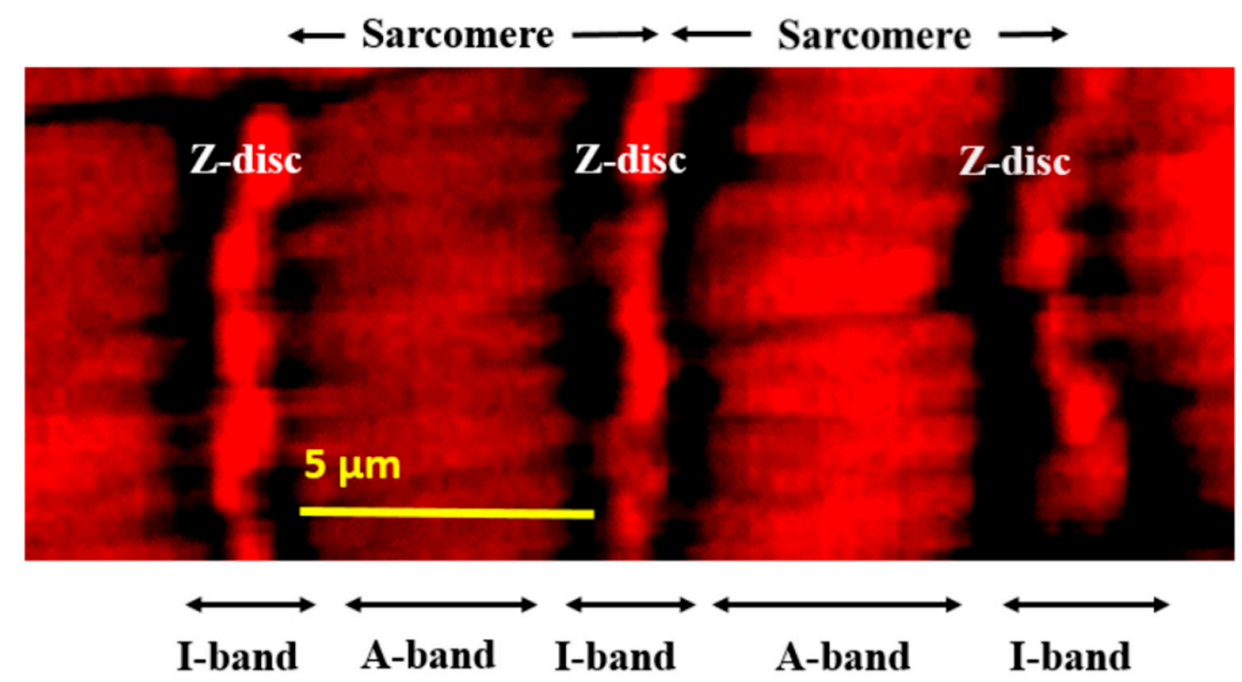

Figure 2. Regular sarcomere units within an oviductal muscle fiber demonstrated at high resolution by fluorescence confocal microscopy and actin staining by phalloidin.

In the mutants, however, the most conspicuous phenotype was the loss of sarcomeres at restrictive temperature, $29^{\circ} \mathrm{C}$ (Figure 3, B4 through G4, Supplementary Figure S2, A4, B4), whereas in wild-type control flies normal sarcomere structure and striation was present at both $20^{\circ} \mathrm{C}$ and $29^{\circ} \mathrm{C}$ (Figure 2 , Figure 3, A1, A4). In mutants at $29^{\circ} \mathrm{C}$, parallel ordered enhanced actin staining intensity areas were present within the muscle fibers that extended over areas larger than a single sarcomere, resembling actin stress fibers or excess actin cross-linking (Figure 3, white rectangles in B4 through G4, Supplementary Figure S2, A4, B4). Beyond these areas, amorphous, intensive actin staining aggregates appeared in the sarcoplasm (Figure 3, white arrows in B4 through G4, Supplementary Figure S2, A4, B4). An additional prominent phenotype of the col4a1 mutants at $29{ }^{\circ} \mathrm{C}$ was the irregular and uneven COL4A1 deposition in the individual muscle fibers (Figure 3, white arrowheads, B5 through G5, Supplementary Figure S2, A5, B5), while in wild-type controls homogenous COL4A1 staining was present at $29^{\circ} \mathrm{C}$ (Figure 3, A5). In the isoallelic mutants col4a1 $1^{\text {G552D2 }}$ and col4a1 ${ }^{\text {G1025E2 }}$ the same COL4A1 staining pattern was observed (Supplementary Figure S2) as in the other lines of the allelic series (Figure 3). Muscle fibers of the common oviduct in wild-type animals harbor morphologic features of the striated muscles, present the regular register of I and A bands and sarcomeres are bordered by Z-discs (Figure 1, Figure 3, A1-A6). We therefore conclude that the compromised sarcoplasmic morphology and uneven COL4A1 staining/localization is a general phenotype of our series of col4a1 mutations.

The control flies do not alter their actin accumulation in the muscle fibers at permissive or restrictive temperatures, as measured by quantitative fluorescence confocal microscopy. The presence of col4a1 mutation seems to exert a non-consequent influence on the actin content of the muscle fibers at both permissive or restrictive conditions. Statistically significant downregulation of actin synthesis, compared to wild-type control flies occurred in col4a1 ${ }^{\mathrm{G} 233 E}$, col4a1 ${ }^{\mathrm{G} 552 D 1}$, col4a1 ${ }^{\mathrm{G} 1025 E 2}$ mutants at $20^{\circ} \mathrm{C}$, whereas mutant col4a1 ${ }^{G 1393 E}$ synthesized actin at higher concentration (Figure 4). Under restrictive conditions we observed univocal decrease of actin concentration in col4a1 ${ }^{\text {G552D1, col4a1 }}{ }^{\text {G1043S }}$ and col4a1 ${ }^{\text {G1393E }}$ mutants (Figure 4). 


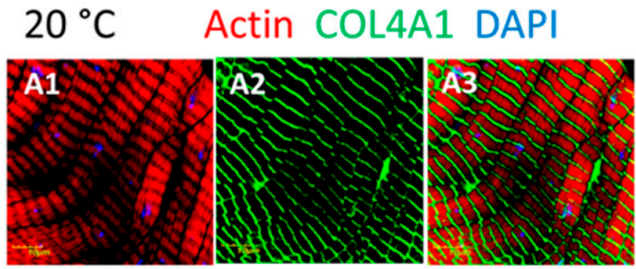

$29^{\circ} \mathrm{C}$
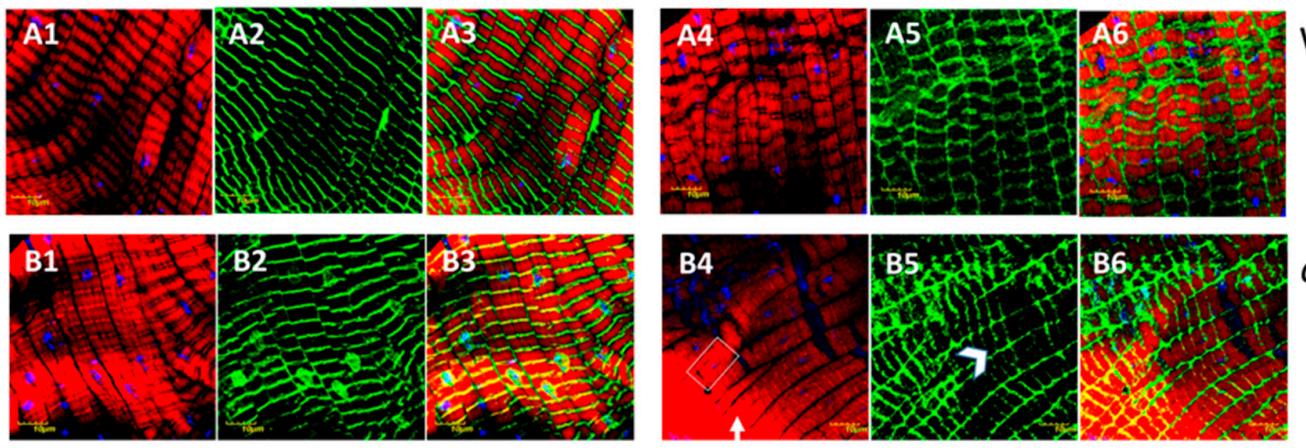

Wild-type
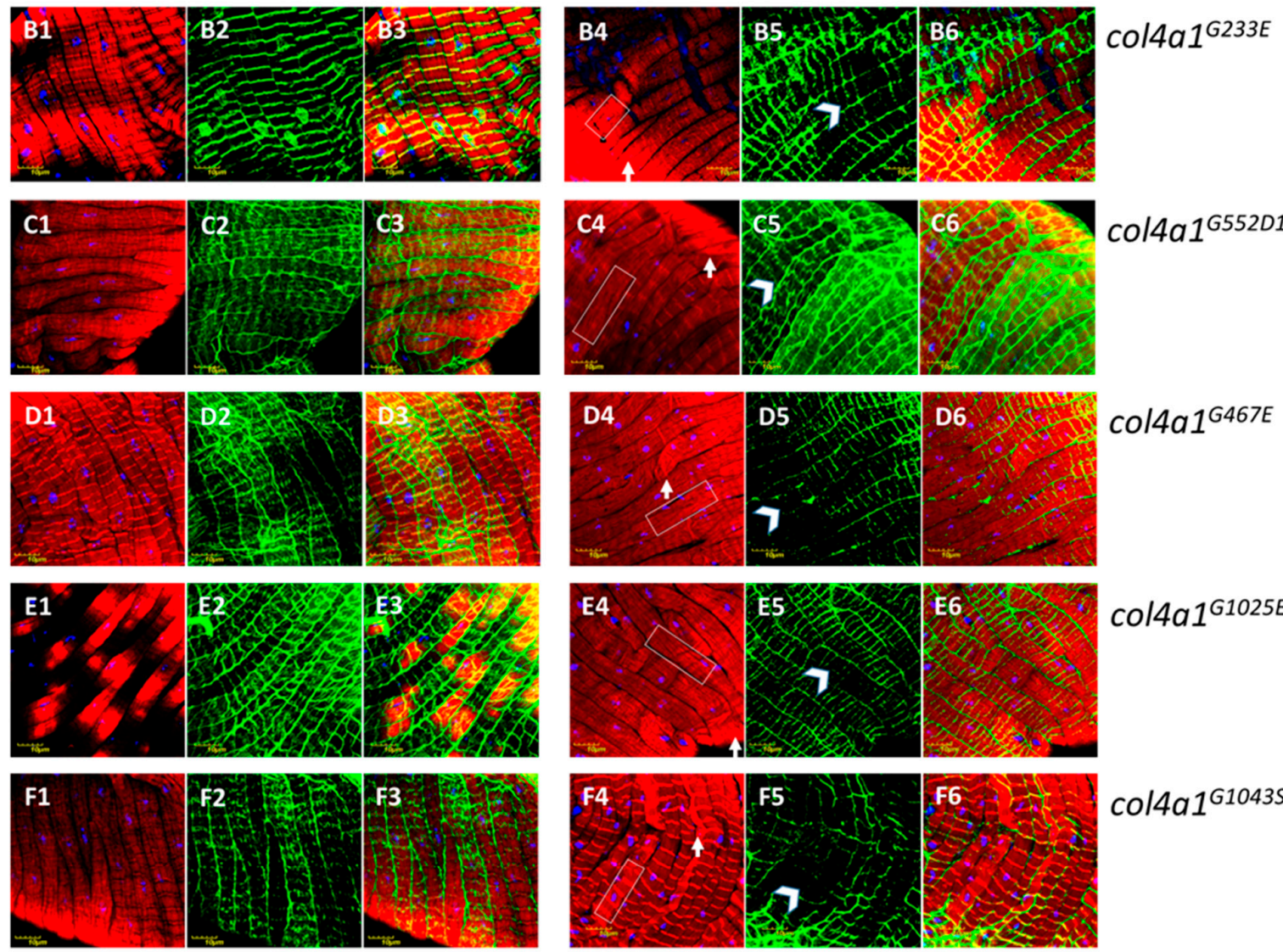

col4a1 G1025E1
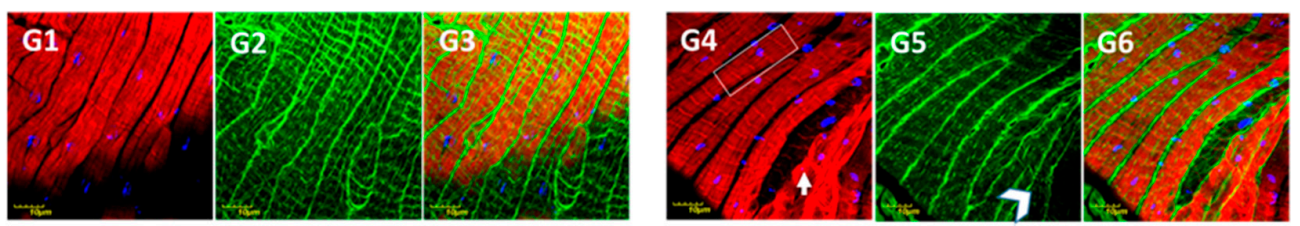

col4a1 $1^{\text {G1043s }}$

Figure 3. Loss of sarcomeres in col4a1 ${ }^{\mathrm{G} 233 \mathrm{E}}$, col4a1 ${ }^{\mathrm{G} 467 \mathrm{E}}$, col4a1 $^{\mathrm{G} 552 \mathrm{D} 1}$, col4a1 ${ }^{\mathrm{G} 1025 \mathrm{E} 1}$, col4a1 ${ }^{\mathrm{G} 1043 \mathrm{~S}}$ and col4a1 ${ }^{\mathrm{G} 1393 \mathrm{E}}$ mutant lines at $29^{\circ} \mathrm{C}$ (B4 through G4) in comparison with wild-type control (A4). Representative actin bundles (white rectangles in B4 through G4), actin aggregates (white arrows in B4 through G4), uneven COL4A1 expression (white arrowheads in B5 through G5). A3 through G3 and A6 through G6: Overlays of actin and COL4A1 staining. Bars, lower left, 10 micrometers.

The mutant col4a1 ${ }^{\mathrm{G} 233 \mathrm{E}}$ accumulates actin at $29^{\circ} \mathrm{C}$, similarly to the col4a1 ${ }^{\mathrm{G} 1025 \mathrm{E} 2}$ allele, whereas the actin concentration in the isoallelic col4a1 $1^{\mathrm{G} 1025 E 1}$ mutant remains unchanged (Figure 1, panel $C^{\prime}$ ). In the rest of the mutants we recorded elevated actin concentration (Figure 1, panel $\mathrm{C}^{\prime}$ ). Either up- or downregulation of actin expression in common oviducts seems disadvantageous given all mutants are female sterile and do not lay eggs at restrictive temperature [9]. 


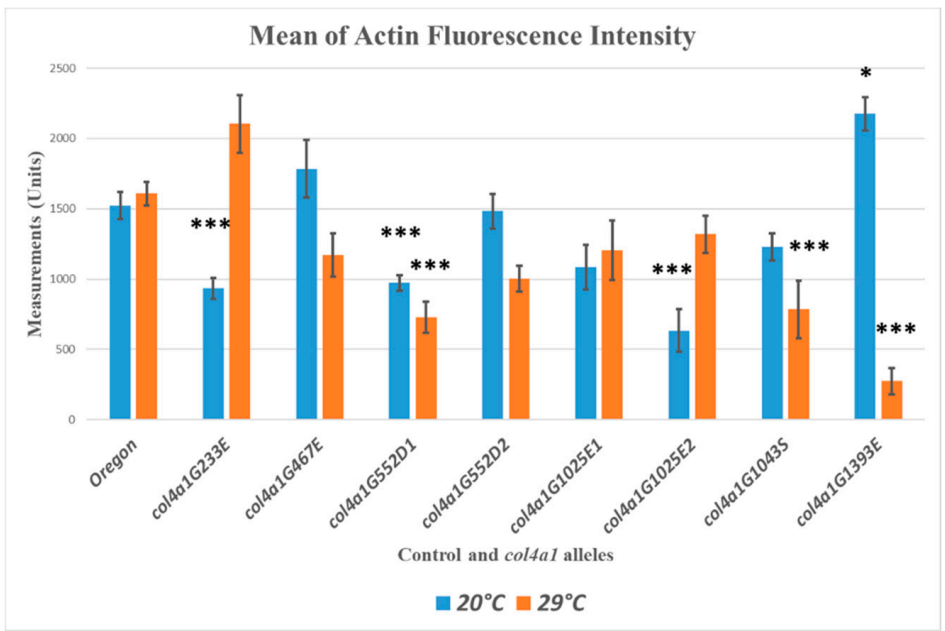

Figure 4. Quantitative measurements of actin content in muscle fibers by fluorescence light intensities of phalloidin labeled muscle. Significance is labelled by asterisk $p<0.05 ;{ }^{* *} p<0.0005$.

\subsection{The Myopathic Phenotype Co-Segregates with the Mutation-Carrying Chromosome}

The head-to-head pair of col4a1 and col4a2 genes localize to the 25C band of the second chromosome in Drosophila. If two different dominant temperature-sensitive (DTS) mutations are present in trans configuration the compound heterozygotes provide viability by interallelic complementation [9]. In order to determine to what extent compound heterozygotes can recapitulate the dominant temperature-sensitive phenotype affecting sarcoplasmic actin morphology, we have generated the col4a1 ${ }^{\text {G233E/G1025E1 double }}$ mutant and its reciprocal pair col4a1 ${ }^{\mathrm{G} 1025 E 1 / \mathrm{G} 233 E}$. In both compound heterozygotes, the sarcomere structure was lost, actin bundles developed (Figure $2 \mathrm{~A}, \mathrm{D}$, white rectangles), intensively staining actin aggregates were deposited (Figure $5 \mathrm{~A}, \mathrm{D}$, white arrows), and the COL4A1 protein was detected in uneven and irregular pattern (Figure $5 \mathrm{~B}, \mathrm{E}$, white arrowheads). These results indicate that in col4a1 ${ }^{+/}$heterozygotes, compromised sarcoplasmic actin morphology and aberrant COL4A1 expression and localization are linked to colta1 mutations, are independent from the genetic context, and are not a secondary effect of increased temperature.

\section{actin COL4A1 DAPI}
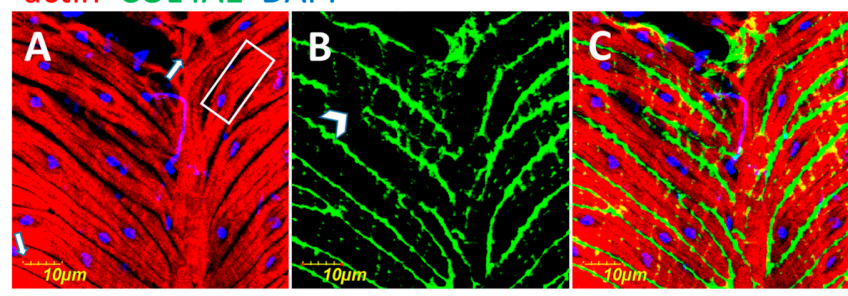

col4a1 ${ }^{G 233 E / G 1025 E 1}$
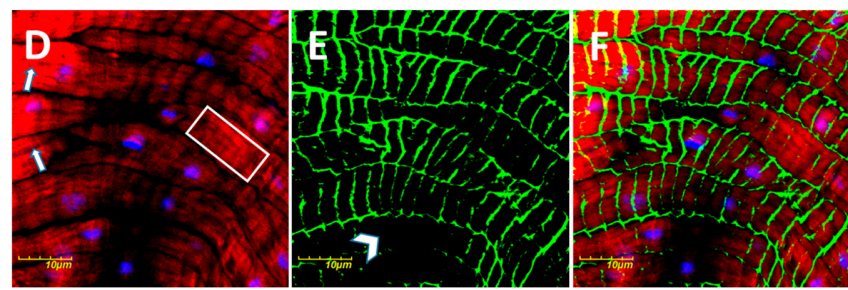

col4a1 $1^{\text {G1025E1/G233E }}$

Figure 5. Sarcomeric loss (A, D), actin bundles (A, D, white rectangles), intensively staining actin aggregates (A, D, white arrows), COL4A1 protein in uneven and irregularly deposited fashion $\left(\mathrm{B}, \mathrm{E}\right.$, white arrowheads) in compound heterozygote col4a1 ${ }^{G 233 E / G 1025 E 1}(\mathrm{~A}-\mathrm{C})$ and in reciprocal col4a1 $^{\text {G1025E1/G233E }}$ (D-F) double mutants. C, F: Overlays of actin and COL4A1 stainings. The experiment was performed at $20{ }^{\circ} \mathrm{C}$. Wild-type control provided in Figure 3, upper row A. Bars, lower left, 10 micrometers. 


\subsection{Z-disc Disintegration, Streaming and Aberrant Integrin Expression in col4a1 Mutants}

In muscles of wild-type Drosophila, integrin is expressed at the muscle attachment sites and appears as punctate staining at the costameres aligned with Z-discs [23]. In order to determine the exact position of the Z-discs in the muscle fibers of the oviduct, we used antibodies against the scaffold protein kettin as a morphological marker. In wild-type controls kettin staining appeared as parallel-ordered lines in each fiber perpendicular to the long axis delineating the sarcomeres and proper striation at both permissive and restrictive temperatures (Figure $3 \mathrm{~A} 1, \mathrm{~A} 4$ ). Immunohistochemistry using anti-integrin antibodies provided the same staining pattern in close localization as observed for kettin (Figure 6 A2,A5, and overlays (Figure 6 A3,A6), confirming integrin localization to the Z-discs in muscle fibers of the oviduct.

In col4a1 mutant, we observed aberrant integrin expression in the epithelial cells of the Malpighian tubules [30], and also surmised irregular integrin deposition in muscle fibers. In mutant oviductal muscle fibers the Z-disc structure, delineated by integrin expression, was disrupted and formed a zig-zag pattern, Z-disc material appeared torn across a large part of sarcomere, and integrin staining was deposited randomly within the sarcomere as dots, consistent with the muscle pathology of Z-disc streaming (Figure 6, white arrows, panels B-D), in which Z-disc structure is disrupted and Z-disc material appears ripped out and deposited within the sarcomere (Figure 6, yellow arrows, panels B-D). These phenotypic features were enhanced by incubating the mutants at restrictive temperature: Areas expressing excess integrin (Figure 6, panels B-D, red arrows), or depositing integrin scarcely (Figure 6, panels B-D, blue arrows). Z-disc streaming, intrasarcomeric integrin deposition and uneven expression seems to be a general feature of col4a1 mutants, as the same phenotype was observed in the rest of the members of the allelic series (Supplementary Figure S3).

We have recently reported a further phenotypic manifestation of the mutants, the ectopic assembly and transition of Z-discs the anisotropic (A) band at the level of M-discs at statistical significance in the mutants [32]. These observations collectively suggest the requirement of proper COL4A1 protein in regular integrin expression and explain female sterility of the mutants that do not lay eggs at $29^{\circ} \mathrm{C}$ [9].

$20^{\circ} \mathrm{C}$ kettin integrin

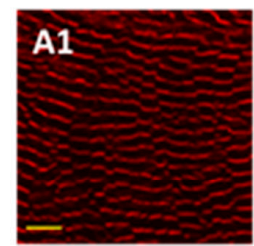

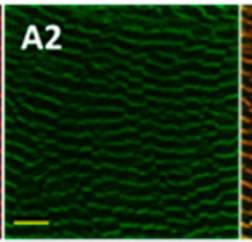

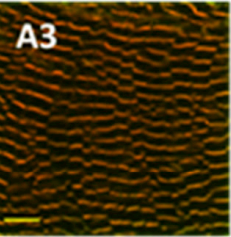

Wild-type control

$20^{\circ} \mathrm{C}$ integrin $29^{\circ} \mathrm{C}$

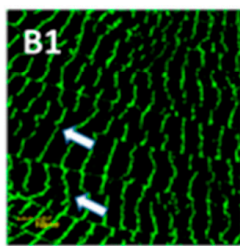

col4a1 ${ }^{\text {G233E }}$

col4a1 $1^{\text {G467E }}$

\section{$29^{\circ} \mathrm{C}$}
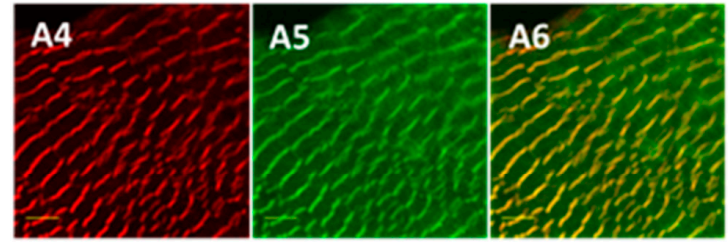

$20^{\circ} \mathrm{C}$ integrin $29^{\circ} \mathrm{C}$

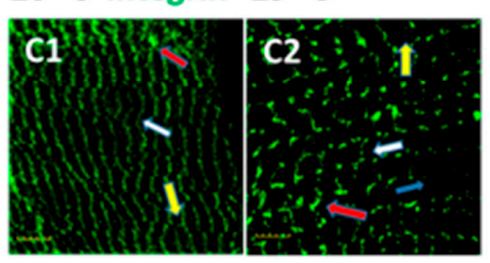

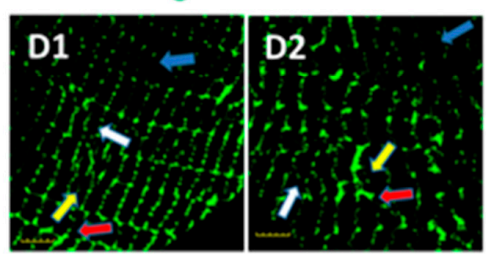

col4a1655201

Figure 6. Staining of muscle fibers of common oviduct by the Z-disc marker kettin and integrin in wild-type control (A1-A6) at permissive and restrictive temperatures. Note unchanged integrin expression at $29^{\circ} \mathrm{C}$ (A5) and close vicinity of kettin and integrin depositions revealed by the complementary color orange in overlays (A3, A6). Panels B, C, D: col4a1 ${ }^{G 233 E}$, col4a1 $^{G 467 E}$, col4a1 $^{\mathrm{G} 552 D 1}$ mutants at permissive and restrictive temperatures. Streaming of the Z-discs (white arrows), integrin expression within the sarcomeres (yellow arrows), excess integrin expression (red arrows) or deficient integrin deposition (blue arrows) are noted in the mutants (panels B-D). Bars, lower left, 10 micrometers. 


\subsection{Fiber Atrophy and Fiber Size Diversity}

We measured the diameter of the individual muscle fibers and the most frequent value was found to correspond to $8 \mu \mathrm{m}$ both in mutant col4a1 ${ }^{G 233 E}$ line and wild-type (Oregon) controls, incubated at permissive temperature (Figure 7). Incubation of mutant animals at $29^{\circ} \mathrm{C}$ shifted the diameters of the muscle fibers toward smaller values. We observed the same phenomenon in the rest of the lines of the allelic series (Supplementary Figure S4). The ratio of the muscle fibers with diameters below $8 \mu \mathrm{m}$ up to $4 \mu \mathrm{m}$ increased two-threefold, by $12 \%-34 \%$ in the mutants, whereas the same ratio in control flies remained $30 \%$ at both temperatures with the majority of fiber diameters in the range of 7-8 $\mu \mathrm{m}$ and only $4 \%-6 \%$ of $6 \mu \mathrm{m}$ as the smallest value (Figure 7, Supplementary Figure S4, Table 2). These data showed size heterogeneity, wasting and atrophy of muscle fibers, characteristic features of dystrophic muscle in the col4a1 mutant lines.
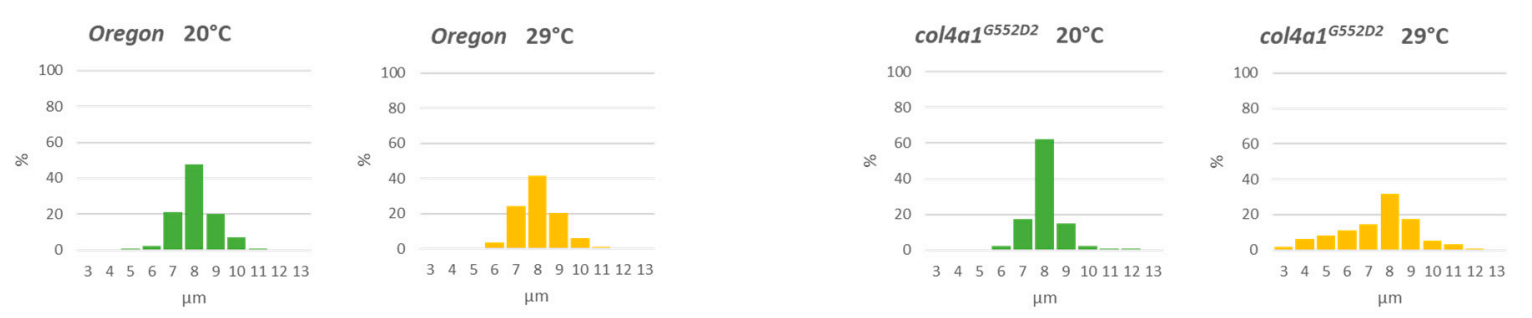

Figure 7. Muscle fiber atrophy measured by the reduced diameter of the fibers in the mutants at $29^{\circ} \mathrm{C}$.

Table 2. Summary of the phenotypes of the col4a1 mutants.

\begin{tabular}{|c|c|c|c|c|c|c|c|c|c|}
\hline Allele & $\operatorname{col}^{\prime} a 1^{G 233 E}$ & col4a1 ${ }^{G 467 E}$ & $\operatorname{col}_{a} a 1^{G 552 D 1}$ & $\operatorname{col}_{4 a 1^{G 552 D 2}}$ & $\operatorname{col}_{a} 1^{G 1025 E 1}$ & $\operatorname{col}_{4 a 1^{G 1025 E 2}}$ & col4a1 $1^{\text {G1043S }}$ & $\operatorname{col} a 1^{G 1393 E}$ & OreR, wt \\
\hline Sarcomere loss & + & + & + & + & + & + & + & + & - \\
\hline $\begin{array}{c}\text { Z-disc } \\
\text { streaming }\end{array}$ & + & + & + & + & + & + & + & + & - \\
\hline $\begin{array}{l}\text { Irregular } \\
\text { integrin } \\
\text { expression }\end{array}$ & + & + & + & + & + & + & + & + & - \\
\hline $\begin{array}{c}\text { Amorphic actin } \\
\text { deposition }\end{array}$ & + & + & + & + & + & + & + & + & - \\
\hline $\begin{array}{l}\text { Atrophy, } \\
\text { fibers with } \mathrm{D}<8 \\
\text { micrometer at } \\
20^{\circ} \mathrm{C}\end{array}$ & $10 \%$ & $10 \%$ & $14 \%$ & $14 \%$ & $14 \%$ & $18 \%$ & $16 \%$ & $8 \%$ & $30 \%$ \\
\hline $\begin{array}{c}\text { Fiber size } \\
\text { disproportion }\end{array}$ & + & + & + & + & + & + & + & + & - \\
\hline $\begin{array}{l}\text { Uneven } \\
\text { COL4A1 } \\
\text { expression }\end{array}$ & + & + & + & + & + & + & + & + & - \\
\hline
\end{tabular}

\section{Conclusions}

In our mutant series, glycine substitutions by large, charged or polar amino acids, glutamate, aspartate and serine, occurred within the col4a1 gene by transition of the second guanine nucleotide to adenine consistent with the mutagen EMS. Two isoallelic variants col4a1 G552D1, col4a1 ${ }^{\text {G552D2}}$, and $c 0 l 4 a 1^{G 1025 E 1}$, col4a1 ${ }^{G 1025 E 2}$ alleles were identified. The importance of the Gly552 residue is reflected by the fact that a recent EMS mutagenesis resulted in the isolation of the same, temperature-sensitive, dominant-negative col4a1 ${ }^{G 552 D}$ allele [33]. Genotype-phenotype relationships explored in over hundred COL4A1 mutants identified in patients and in murine models revealed that the position of the mutation and not the biochemical properties of the substituting amino acid seems to have a greater impact on the phenotype and disease severity [34]. In our Drosophila mutant series, however, regardless of 
the position of the mutation within the collagenous domain of the colta1 gene, we observed similar phenotypic defects.

As the oviduct, and also the larval body wall muscle phenotype [9], compromised actin organization and deposition, loss of sarcomere structure were noted in all alleles studied, features that are common in myopathic or dystrophic conditions, with disintegrating muscle sarcomeres together with disintegration and streaming of Z-discs [35]. These morphologic changes impact the function of the common oviduct as females become sterile and do not lay eggs [9]. Conditional knockdown of genes in Drosophila, involved genes in integrin mediated adhesion, including talin, alpha-actinin, integrin-linked kinase, alpha PS2 and beta PS integrins result in the common phenotype of Z-disc streaming [26], similar to our col4a1 mutant series, indicating functional interdependence. Similarly, Walker-Warburg Syndrome is diagnosed as a monogenic trait in patients carrying mutations in several genes [19,36]. Importantly, in Drosophila mutants defective in protein O-mannosyltransferases the symptoms of the Walker-Warburg Syndrome were identified; as part of the mutant phenotypes Z-disc streaming, actin filament disorganization and bundle formation were reported also [36].

The human myopathic/dystrophyc conditions marked by Z-disc streaming and sarcomeric disorganization involves genes that encode components of integrin-mediated adhesion markedly by genetic reasons. The $I k^{-/}$mouse embryos die during peri-implantation stage due to impaired epiblast polarization and F-actin accumulation at integrin attachment sites [37]. Knockout mutants for the integrin beta subunits and for majority of the alpha subunits have been constructed with phenotypes ranging from a complete block in preimplantation, through developmental defects to perinatal lethality, demonstrating the specificity of each integrin. Muscular dystrophy was observed in patients with ITGA5 or ITGA7 mutations [8]. Z-disc streaming, however, was not reported in association of ITGA5 or ITGA7 mutations. Mouse mutants of talin 1 or talin 2 perform myopathy and disassembly of the sarcomeres [38]. However, the embryonic lethal mutation in the Drosophila rhea gene encoding talin recapitulate the phenotype of the integrin beta PS mutations, demonstrating their functional similarities [25]. These results indicate that genes involved in integrin-mediated adhesion are essential, their homozygous recessive or null mutations are often lethal. The conditional lethality of the temperature-sensitive, heterozygous col4a1 Drosophila mutant series allowed manifestation of phenotypic elements that would be non-explorable in humans or mice, such as the disrupted sarcomeric cytoarchitecture and Z-disc streaming that support a role for COL4A1 in integrin mediated adhesion. In conclusion, our Drosophila mutant series may serve as an effective model to uncover the mechanisms by which COL4A1 mutations result in disrupted myofiber-basement membrane interactions and compromised muscle function and provide biomarkers to explore during therapeutic approaches.

\section{Materials and Methods}

\subsection{PCR Amplification and Sequencing}

The algorithm of Primerfox was used to design sequence specific primers for the coltal gene (Table 3). The amplification reaction was carried out with the aid of KAPA Taq polymerase and Fermentas dNTP mix (Thermo Scientific, Vilnius, Lithuania) guided by a touchdown PCR protocol. The initial denaturation at $94{ }^{\circ} \mathrm{C}$ lasted for $150 \mathrm{~s}$, followed by 30 cycles of $93^{\circ} \mathrm{C}$ for $15 \mathrm{~s}$, then $65^{\circ} \mathrm{C}$ $\left(-0.6{ }^{\circ} \mathrm{C} / \mathrm{cycle}\right)$ for $15 \mathrm{~s}$ and $72{ }^{\circ} \mathrm{C}$ for $45 \mathrm{~s}$. The final elongation step at $72{ }^{\circ} \mathrm{C}$ was allowed to run for $180 \mathrm{sec}$. The lengths of the products were checked on $1 \%$ agarose gel, followed by cleanup on silica columns (ZenonBio, Szeged, Hungary). The DNA samples containing the appropriate primers were sent to Eurofins Genomics for sequencing. The same PCR fragments originating from different reactions were read multiple times in both directions to ensure reliability of the results. The received sequence information was aligned to the database of NCBI with the Blast algorithm. 
Table 3. List of PCR primers used for sequencing the col4a1 gene.

\begin{tabular}{cc}
\hline Name & Sequence \\
\hline F1a & CACGGATAGTGTACATGAGC \\
\hline F2a & GCCTTTAGCAAACTCTCTTG \\
\hline F3 & TCCTCGTTTCCCGTCAAACC \\
\hline F4 & TTCAAGGGCAATGCTGGTGC \\
\hline F5 & GGTCTCAATGGTCTGCAAGG \\
\hline F6 & TCCCGGAATGGATGGTTTGC \\
\hline F7 & GAAGGGTGAACCAGGAATGC \\
\hline F8 & TCTGTTGGATACTGCGTAGC \\
\hline R1 & CATAGCTCTCTTCGATTGGC \\
\hline R2 & CTCCCTTCTGTCCCATATCG \\
\hline R3 & CCTTGATACCCATGTCTCCC \\
\hline R4 & AAACCAATGGGTCCGGTTGG \\
\hline R5 & TCACCAGGATAGCCAACAGC \\
\hline R6 & TCTCCCTTAGGTCCATTGCG \\
\hline R7 & CGGCAGTGTGCTATTATAGG \\
\hline R8 & GCATTGTTTCGCATTTAATCGG
\end{tabular}

\subsection{Maintenance of Drosophila Strains}

Wild-type Oregon flies and col4a1 mutant stocks were maintained at $20{ }^{\circ} \mathrm{C}$ and $29{ }^{\circ} \mathrm{C}$ on yeast-cornmeal-sucrose-agar food, completed with the antifungal nipagin. The mutant stocks were kept heterozygous over the CyRoi balancer chromosome that prevents recombination with the mutation-carrying homolog, thus the genotype of the mutants presented here is: $c o l 4 a 1^{-}+/ \mathrm{col}_{4 a 1^{+}} \mathrm{CyRoi}$.

Common oviducts were removed under carbon dioxide anesthesia from adults that were grown at both permissive and restrictive temperature for 14 days. Dissected common oviducts were fixed in $4 \%$ paraformaldehyde dissolved in phosphate buffered saline (PBS) for $10 \mathrm{~min}$, washed tree times in PBS, permeabilized for $5 \mathrm{~min}$ in $0.1 \%(\mathrm{w} / \mathrm{v})$ Triton $\mathrm{X}$ dissolved in PBS and washed tree times in PBS. Blocking was achieved for in 5\% BSA dissolved in PBS for $1 \mathrm{~h}$, and washed tree times in PBS. Trans-heterozygous strains were generated by crossing two col4a1 $1^{+-}$heterozygotes selecting for the loss of the balancer chromosome CyRoi.

\subsection{Immunostaining and Antibodies}

Nuclei in the dissected common oviducts were counter-stained by $1 \mu \mathrm{g} / \mathrm{mL} 4^{\prime}$,6-diamino-2-phenylindol

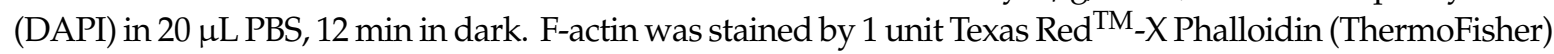
in $20 \mu \mathrm{L}$ PBS for $20 \mathrm{~min}$. Integrin dimer staining was achieved by an equimolar mixture consisting of both anti-integrin monoclonal antibodies (mouse, Developmental Studies Hybridoma Bank) that recognize alpha PS I or alpha PS II subunits. Mouse antibody against Drosophila COL4A1 protein was generated by Creative Ltd, Szeged, Hungary. Primary mouse antibodies were visualized by $1 \mu \mathrm{L} \mathrm{F}\left(\mathrm{ab}^{\prime}\right)$ 2-Goat Anti-Mouse IgG $(\mathrm{H}+\mathrm{L})$ Cross Adsorbed Secondary Antibody conjugated with Alexa Fluor 488 (ThermoFisher) in $20 \mu \mathrm{L}$ PBS for $1 \mathrm{~h}$ or $1 \mu \mathrm{L}$ Goat Anti-Mouse IgG (H+L) Cross Adsorbed Secondary Antibody, Alexa Fluor 350, in $20 \mu \mathrm{L}$ PBS for $1 \mathrm{~h}$.

\subsection{Confocal Microscopy}

Photomicrographs of the common oviducts were generated by confocal laser scanning fluorescence microscopy (Olympus Life Science Europa GmbH, Hamburg, Germany). Microscope configurations were set up as described [29]. Briefly, objective lens: UPLSAPO 60x (water, NA: 0.90); sampling speed: $8 \mu \mathrm{s} /$ pixel; line averaging: 2x; scanning mode: sequential unidirectional; excitation: $405 \mathrm{~nm}$ (DAPI), 
$543 \mathrm{~nm}$ (Texas Red) and $488 \mathrm{~nm}$ (Alexa Fluor 488); laser transmissivity: 7\% were used for DAPI, 15\% for Alexa Fluor 488 and 20\% for Texas Red.

Quantitative evaluation of the fluorescence light intensities of the $543 \mathrm{~nm}$ Texas Red signals was achieved by the FLUOVIEW FV1000 program of Olympus, system version 4.2.1.20. We generated $\sim 20$ independent images for each wild-type control and mutant samples thus analyzing over 400 photomicrographs. Statistical analysis was carried out by analyzing raw confocal fluorescent light intensities. Signal amplification was applied in images for morphologic analysis.

\subsection{Size Determination of the Muscle Fibers}

Confocal photomicrographs displaying oviducts were stained by Texas Red ${ }^{\mathrm{TM}}-\mathrm{X}$ Phalloidin and anti-COL4A1 antibody, taken from all mutants and wild-type controls at 20 and $29^{\circ} \mathrm{C}$. Diameters of hundred randomly chosen muscle fibers were measured generating altogether 9000 values. Diameters were calculated as distances between the lateral sides of the individual muscle fibers, perpendicular to the long axis, in areas with unchanged diameters. Bins of diameter intervals differing by one $\mu \mathrm{m}$ were displayed in histograms showing the numbers of the corresponding diameters.

Supplementary Materials: Supplementary materials can be found at http://www.mdpi.com/1422-0067/20/20/ 5124/s1.

Author Contributions: Conceived and designed the experiments: M.M. Performed the experiments: A.A.K., N.S.-P., M.K., M.M. Analyzed the data: A.A.K., N.S.-P., M.M. Provided resources: Z.B. Developed manuscript: M.M., K.C., Z.B. Supervision, project administration: M.M.

Funding: This research was supported by the Hungarian Scientific Research Fund OTKA, contract nr. NN 108283 to M.M. and by the UNKP-18-3 New National Excellence Program of the Ministry of Human Capacities, contract nr. UNKP-18-3-IV-SZTE-23 to A.A.K and UNKP-18-3-IV-SZTE-43 to N.S-P.

Conflicts of Interest: The authors declare that there is no conflict of interests regarding the publication of this paper.

\section{References}

1. Pozzi, A.; Yurchenco, P.D.; Iozzo, R.V. The nature and biology of basement membranes. Matrix Biol. 2017, 57-58, 1-11. [CrossRef] [PubMed]

2. Sanes, J.R. The Basement Membrane/Basal Lamina of Skeletal Muscle. J. Biol. Chem. 2003, 278, 12601-12604. [CrossRef] [PubMed]

3. Guicheney, P.; Vignier, N.; Helbling-Leclerc, A.; Nissinen, M.; Zhang, X.; Cruaud, C.; Lambert, J.-C.; Richelme, C.; Topaloglu, H.; Merlini, L.; et al. Genetics of laminin alpha 2 chain (or merosin) deficient congenital muscular dystrophy: From identification of mutations to prenatal diagnosis. Neuromuscul. Disord. 1997, 7, 180-186. [CrossRef]

4. Durbeej, M.; Campbell, K.P. Muscular dystrophies involving the dystrophin-glycoprotein complex: An overview of current mouse models. Curr. Opin. Genet. Dev. 2002, 12, 349-361. [CrossRef]

5. Lampe, A.K.; Bushby, K.M. Collagen VI related muscle disorders. J. Med. Genet. 2005, 42, 673-685. [CrossRef]

6. Mayer, U.; Saher, G.; Fassler, R.; Bornemann, A.; Echtermeyer, F.; von der Mark, H.; Miosge, N.; Pösch, E.; von der Mark, K. Absence of integrin alpha-7 causes a novel form of muscular dystrophy. Nat. Genet. 1997, 17, 318-323. [CrossRef]

7. Bertini, E.; D'Amico, A.; Gualandi, F.; Petrini, S. Congenital Muscular Dystrophies: A Brief Review. Semin. Pediatr. Neurol. 2011, 18, 277-288. [CrossRef]

8. Hynes, R.O. Integrins: Bidirectional Allosteric Signaling Machines. Cell 2002, 110, 673-687. [CrossRef]

9. Kelemen-Valkony, I.; Kiss, M.; Csiha, J.; Kiss, A.; Bircher, U.; Szidonya, J.; Maróy, P.; Juhász, G.; Komonyi, O.; Csiszár, K.; et al. Drosophila basement membrane collagen col4a1 mutations cause severe myopathy. Matrix Biol. 2012, 31, 29-37. [CrossRef]

10. Alport, A.C. Hereditary familial congenital haemorrhagic nephritis. Br. Med. J. 1927, 1, 504-506. [CrossRef]

11. Hudson, B.G.; Tryggvason, K.; Sundaramoorthy, M.; Neilson, E.G. Alport's Syndrome, Goodpasture's Syndrome, and Type IV Collagen. N. Engl. J. Med. 2003, 348, 2543-2556. [CrossRef] [PubMed] 
12. Zhou, J.; Mochizuki, T.; Smeets, H.; Antignac, C.; Laurila, P.; de Paepe, A.; Tryggvason, K.; Reeders, S.T. Deletion of the Paired Alpha 5(IV) and Alpha 6(IV) Collagen Genes in Inherited Visceral Muscle Tumors. Science 1993, 261, 1167-1169. [CrossRef] [PubMed]

13. Gould, D.B.; Phalan, F.C.; Breedveld, G.J.; van Mil, S.E.; Smith, R.S.; Schimenti, J.C.; Aguglia, U.; van der Knaap, M.S.; Heutink, P.; John, S.W. Mutations in Col4a1 Cause Perinatal Cerebral Hemorrhage and Porencephaly. Science 2005, 308, 1167-1171. [CrossRef] [PubMed]

14. van Agtmael, T.; Schlötzer-Schrehardt, U.; McKie, L.; Brownstein, D.G.; Lee, A.W.; Cross, S.H.; Sado, Y.; Mullins, J.J.; Pöschl, E.; Jackson, I.J. Dominant Mutations of Col4a1 Result in Basement Membrane Defects Which Lead to Anterior Segment Dysgenesis and Glomerulopathy. Hum. Mol. Genet. 2005, 14, 3161-3168. [CrossRef] [PubMed]

15. Favor, J.; Gloeckner, C.J.; Janik, D.; Klempt, M.; Neuhäuser-Klaus, A.; Pretsch, W.; Schmahl, W.; Quintanilla-Fend, L. Type IV Procollagen Missense Mutations Associated with Defects of the Eye, Vascular Stability, the Brain, Kidney Function and Embryonic or Postnatal Viability in the Mouse, Mus Musculus: An Extension of the Col4a1 Allelic Series and the Identification of the First Two Col4a2 Mutant Alleles. Genetics 2007, 175, 725-736. [PubMed]

16. Kuo, D.S.; Labelle-Dumais, C.; Gould, D.B. COL4A1 and COL4A2 Mutations and Disease: Insights into Pathogenic Mechanisms and Potential Therapeutic Targets. Hum. Mol. Genet. 2012, 21, R97-R110. [CrossRef]

17. Plaisier, E.; Gribouval, O.; Alamowitch, S.; Mougenot, B.; Prost, C.; Verpont, M.C.; Marro, B.; Desmettre, T.; Cohen, S.Y.; Roullet, E.; et al. COL4A1 Mutations and Hereditary Angiopathy, Nephropathy, Aneurysms, and Muscle Cramps. N. Engl. J. Med. 2007, 357, 2687-2695. [CrossRef]

18. Alamowitch, S.; Plaisier, E.; Favrole, P.; Prost, C.; Chen, Z.; Van Agtmael, T.; Marro, B.; Ronco, P. Cerebrovascular Disease Related to COL4A1 Mutations in HANAC Syndrome. Neurology 2009, 73, 1873-1882. [CrossRef]

19. Labelle-Dumais, C.; Dilworth, D.J.; Harrington, E.P.; De Leau, M.; Lyons, D.; Kabaeva, Z.; Manzini, M.C.; Dobyns, W.B.; Walsh, C.A.; Michele, D.E.; et al. COL4A1 Mutations Cause Ocular Dysgenesis, Neuronal Localization Defects, and Myopathy in Mice and Walker-Warburg Syndrome in Humans. PLoS Genet. 2011, 7, e1002062. [CrossRef]

20. Guiraud, S.; Migeon, T.; Ferry, A.; Chen, Z.; Ouchelouche, S.; Verpont, M.C.; Sado, Y.; Allamand, V.; Ronco, P.; Plaisier, E. HANAC Col4a1 Mutation in Mice Leads to Skeletal Muscle Alterations due to a Primary Vascular Defect. Am. J. Pathol. 2017, 187, 505-516. [CrossRef]

21. Parkin, J.D.; San Antonio, J.D.; Pedchenko, V.; Hudson, B.; Jensen, S.T.; Savige, J. Mapping Structural Landmarks, Ligand Binding Sites, and Missense Mutations to the Collagen IV Heterotrimers Predicts Major Functional Domains, Novel Interactions, and Variation in Phenotypes in Inherited Diseases Affecting Basement Membranes. Hum Mutat. 2011, 32, 127-143. [CrossRef] [PubMed]

22. Plaisier, E.; Chen, Z.; Gekeler, F.; Benhassine, S.; Dahan, K.; Marro, B.; Alamowitch, S.; Paques, M.; Ronco, P. Novel COL4A1 Mutations Associated with HANAC Syndrome: A Role for the Triple Helical CB3[IV] Domain. Am. J. Med. Genet. A 2010, 152A, 2550-2555. [CrossRef] [PubMed]

23. Rui, Y.; Bai, J.; Perrimon, N. Sarcomere Formation Occurs by the Assembly of Multiple Latent Protein Complexes. PLoS Genet. 2010, 6, e1001208. [CrossRef] [PubMed]

24. Volk, T.; Fessler, L.I.; Fessler, J.H. A role for integrin in the formation of sarcomeric cytoarchitecture. Cell 1990, 63, 525-536. [CrossRef]

25. Brown, N.H.; Gregory, S.L.; Rickoll, W.L.; Fessler, L.I.; Prout, M.; White, R.A.; Fristrom, J.W. Talin Is Essential for Integrin Function in Drosophila. Dev. Cell. 2002, 3, 569-579. [CrossRef]

26. Perkins, A.D.; Ellis, S.J.; Asghari, P.; Shamsian, A.; Moore, E.D.; Tanentzapf, G. Integrin mediated adhesion maintains sarcomeric integrity. Dev. Biol. 2010, 338, 15-27. [CrossRef]

27. Kelemen-Valkony, I.; Kiss, M.; Csiszar, K.; Mink, M. Inherited Myopathies. In Myopathies: New Research; Washington, H.S., Jimenez, C.E.C., Eds.; Nova Science Publishers: Hauppauge, NY, USA, 2012; ISBN 9781622573721.

28. Kiss, M.; Kiss, A.A.; Radics, M.; Popovics, N.; Hermesz, E.; Csiszár, K.; Mink, M. Drosophila type IV collagen mutation associates with immune system activation and intestinal dysfunction. Matrix Biol. 2016, 49, 120-131. [CrossRef] 
29. Kiss, A.A.; Popovics, N.; Boldogkői, Z.; Csiszár, K.; Mink, M. 4-Hydroxy-2-nonenal Alkylated and Peroxynitrite Nitrated Proteins Localize to the Fused Mitochondria in Malpighian Epithelial Cells of Type IV Collagen Drosophila Mutants. BioMed Res. Int. 2018, 2018, 3502401. [CrossRef]

30. Kiss, A.A.; Popovics, N.; Szabó, G.; Csiszár, K.; Mink, M. Altered stress fibers and integrin expression in the Malpighian epithelium of Drosophila type IV collagen mutants. Data Brief 2016, 7, 868-872. [CrossRef]

31. Kiss, M.; Kelemen-Valkony, I.; Kiss, A.A.; Kiss, B.; Csiszár, K.; Mink, M. Muscle dystrophy is triggered by type IV collagen alleles affecting integrin binding sites directly or indirectly in Drosophila. Acta Biochim. Pol. 2012, 59, 26.

32. Kiss, A.A.; Somlyai-Popovics, N.; Tubak, V.; Boldogkoi, Z.; Csiszár, K.; Mink, M. Novel Phenotypic Elements of Type IV Collagenopathy Revealed by the Drosophila Model. Appl. Sci. 2019, 9, 2083. [CrossRef]

33. Hollfelder, D.; Frasch, M.; Reim, I. Distinct functions of the laminin $\beta$ LN domain and collagen IV during cardiac extracellular matrix formation and stabilization of alary muscle attachments revealed by EMS mutagenesis in Drosophila. BMC Dev. Biol. 2014, 14, 26. [CrossRef] [PubMed]

34. Jeanne, M.; Gould, D.B. Genotype-phenotype correlations in pathology caused by collagen type IV alpha 1 and 2 mutations. Matrix Biol. 2017, 57-58, 29-44. [CrossRef] [PubMed]

35. Rahimov, F.; Kunkel, L.M. Cellular and molecular mechanisms underlying muscular dystrophy. J. Cell Biol. 2013, 201, 499-510. [CrossRef] [PubMed]

36. Ueyama, M.; Akimoto, Y.; Ichimiya, T.; Ueda, R.; Kawakami, H.; Aigaki, T.; Nishihara, S. Increased Apoptosis of Myoblasts in Drosophila Model for the Walker-Warburg Syndrome. PLoS ONE 2010, 5, e11557. [CrossRef]

37. Sakai, T.; Li, S.; Docheva, D.; Grashoff, C.; Sakai, K.; Kostka, G.; Braun, A.; Pfeifer, A.; Yurchenco, P.D.; Fässler, R. Integrin-linked kinase (ILK) is required for polarizing the epiblast, cell adhesion, and controlling actin accumulation. Genes Dev. 2003, 17, 926-940. [CrossRef]

38. Conti, F.J.; Monkley, S.J.; Wood, M.R.; Critchley, D.R.; Müller, U. Talin 1 and 2 are required for myoblast fusion, sarcomere assembly and the maintenance of myotendinous junctions. Development 2009, 136, 3597-3606. [CrossRef]

(C) 2019 by the authors. Licensee MDPI, Basel, Switzerland. This article is an open access article distributed under the terms and conditions of the Creative Commons Attribution (CC BY) license (http://creativecommons.org/licenses/by/4.0/). 


\section{Supplementary Materials}

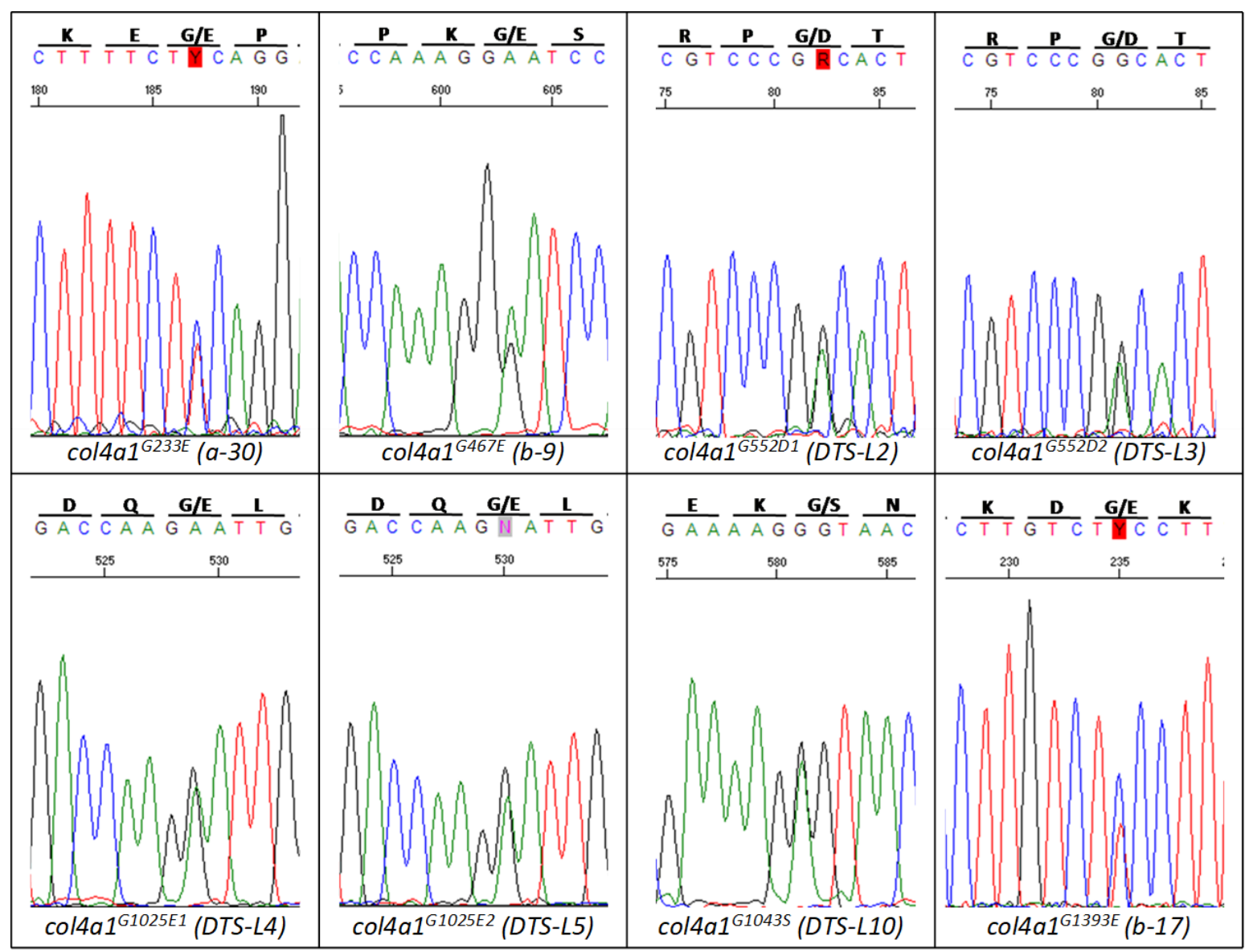

Supplementary Figure S1. Nucleotide sequence of the heterozygous lesions in col4a1 alleles. Note the isoallelic mutations G552D and G1025E. For a better resolution of the heterozygous site the sequence of the complementary strand is displayed in the $b-17$ mutant.
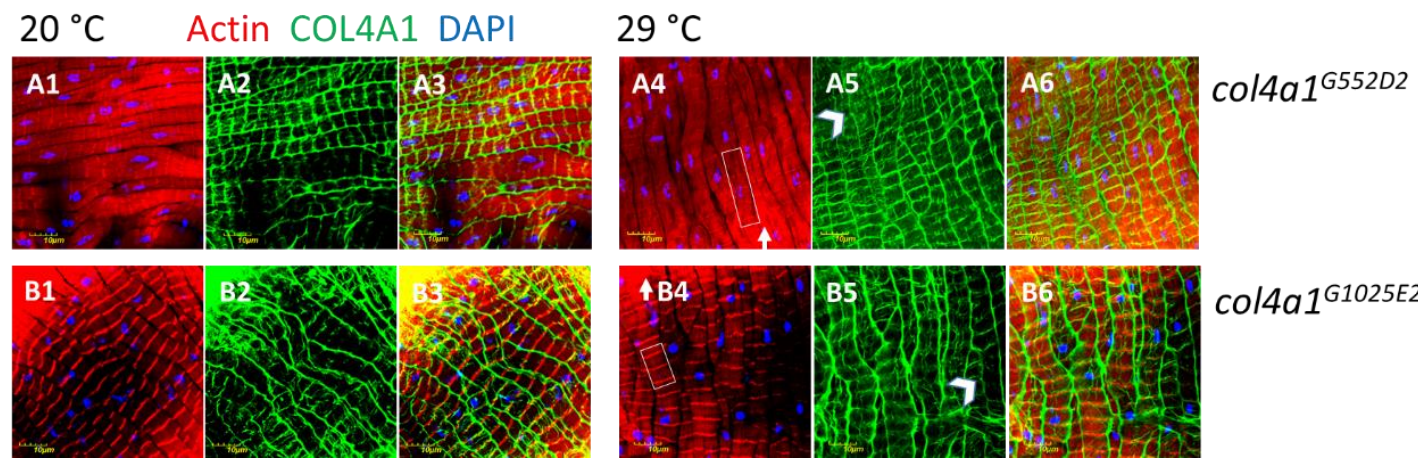

col4a1 G1025E2

Supplementary Figure S2. Loss of sarcomeres (A4, B4) actin bundle development (white rectangles, A4, B4), actin aggregates (white arrows, A4, B4), irregular COL4A1 deposition (white arrowheads, A5, B5) in the isoallelic lines col4a1 $1^{\mathrm{G} 552 D 2}$ and col4a1 $1^{\mathrm{G} 1025 E 2}$. A3, B3, A6, B6: Overlays. Bars, lower left, 10 micrometers. 
$20^{\circ} \mathrm{C}$ integrin $29^{\circ} \mathrm{C}$

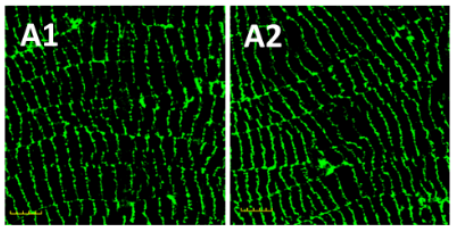

Wild-type control

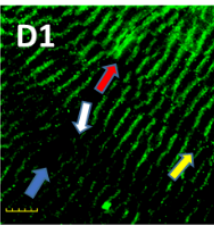

col4a1 ${ }^{\text {G1025E2 }}$ $20^{\circ} \mathrm{C}$ integrin $29^{\circ} \mathrm{C}$

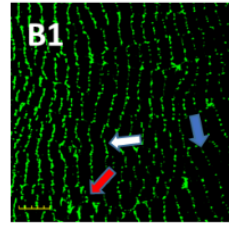

col4a1 $^{\text {G552D2 }}$

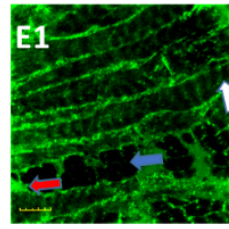

col4a1 $^{\text {G1043s }}$ $20^{\circ} \mathrm{C}$ integrin $29^{\circ} \mathrm{C}$
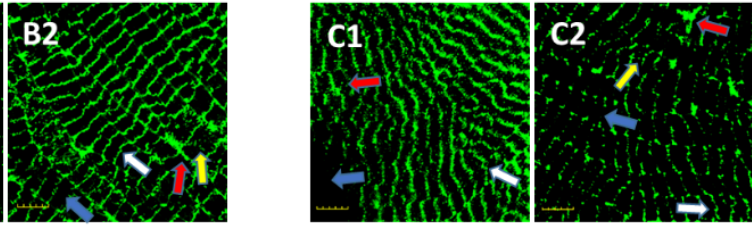

col4a1 $^{\text {G1025E1 }}$

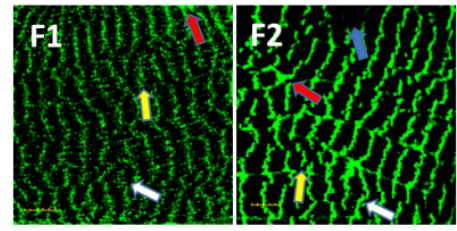

col4a1 $^{\text {G1393E }}$

Supplementary Figure S3. Integrin expression in wild-type control (A) and in col4a1 mutant animals (B-F) at permissive and restrictive temperatures. Streaming of the Z-discs (white arrows), integrin expression within the sarcomeres (yellow arrows), excess integrin expression (red arrows) or deficient integrin deposition (blue arrows) are noted in the mutants (panels B-D). Bars, lower left, 10 micometers. 


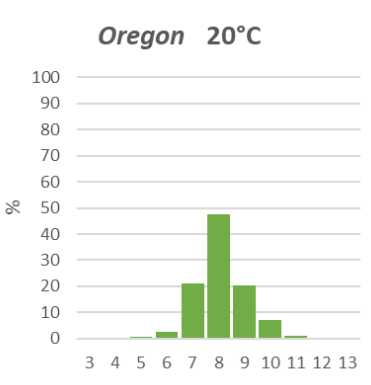

$\mu \mathrm{m}$

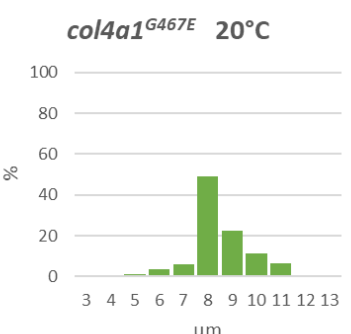

Col4a1 ${ }^{G 1025 E 1} 20^{\circ} \mathrm{C}$

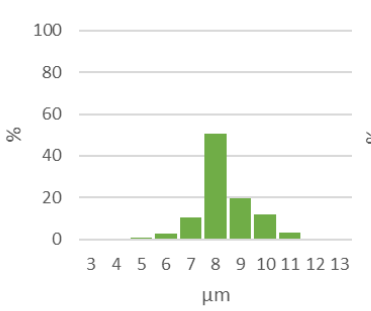

col4a1 ${ }^{610435} 20^{\circ} \mathrm{C}$

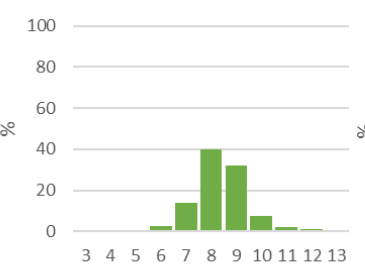

$\mu \mathrm{m}$
Oregon $29^{\circ} \mathrm{C}$

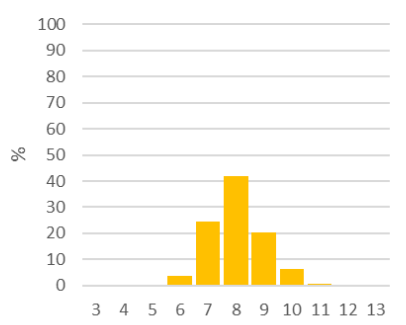

$\mu \mathrm{m}$
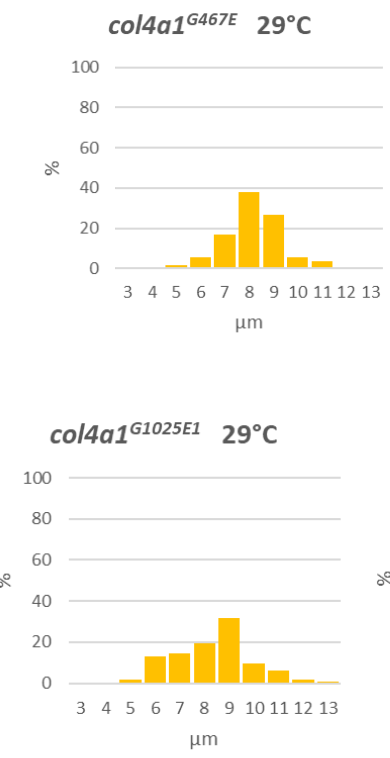

col4a1 ${ }^{\text {G1043s }} 29^{\circ} \mathrm{C}$

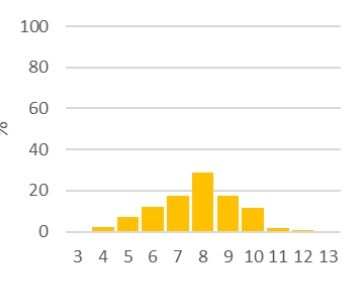

$\mu \mathrm{m}$

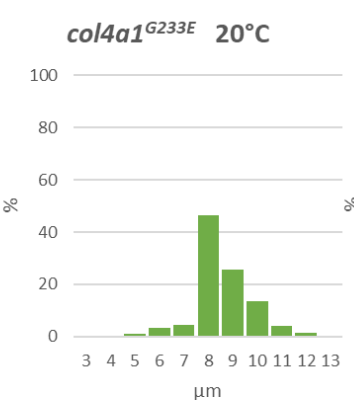

$\mu \mathrm{m}$

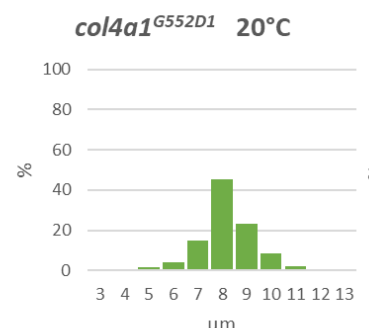

$\mu \mathrm{m}$
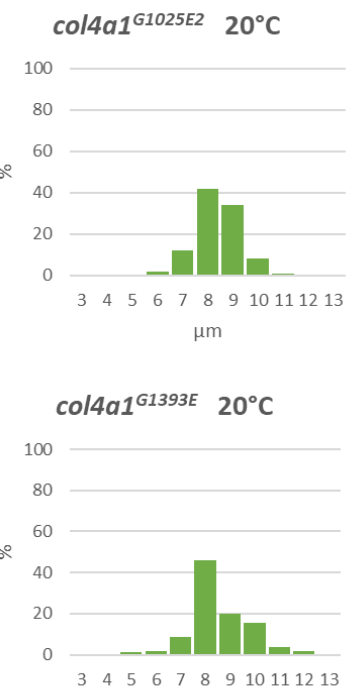

$\mu \mathrm{m}$ col4a1 ${ }^{\mathrm{G} 333} 29^{\circ} \mathrm{C}$

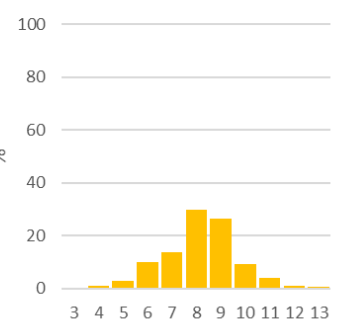

$\mu \mathrm{m}$

col4a1 $1^{G 552 D 1} 29^{\circ} \mathrm{C}$

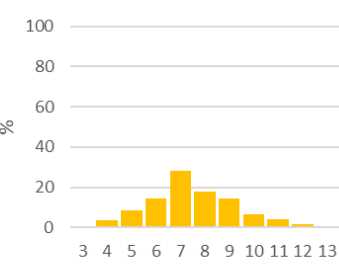

um

\section{col4a1 G1025E2 $29^{\circ} \mathrm{C}$}

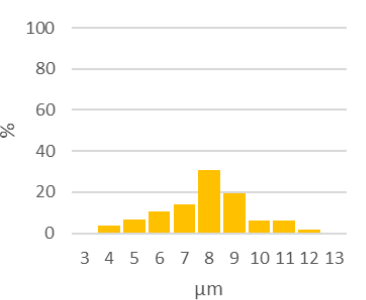

col4a1 ${ }^{\mathrm{G} 1393 \mathrm{E}} 29^{\circ} \mathrm{C}$

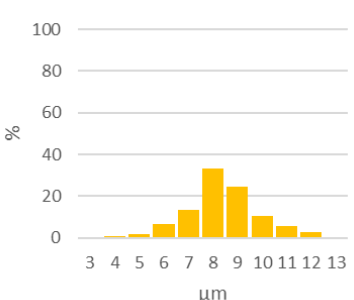

$\mu \mathrm{m}$

Supplementary Figure S4. Size distribution of muscle fibers in col4a1 mutants. Diameters of muscle fibers are shifted toward reduced values (yellow columns) in mutants at $29{ }^{\circ} \mathrm{C}$. 


\section{$\underline{\text { Article VII. (Conference abstract with impact factor) }}$}

Muscle dystrophy is triggered by type IVcollagen alleles affecting integrin binding sites directly or indirectly in Drosophila

Márton Kiss ${ }^{1}$, Ildikó Kelemen-Valkony ${ }^{1}$, $\underline{\text { András Kiss }}{ }^{1}$, Brigitta Kiss ${ }^{2}$, Katalin Csiszár ${ }^{3}$, Mátyás Mink ${ }^{1}$

XXIIIed FECTS and ISMB Joint Meeting, August 25th-29th, 2012, Katowice, Poland

http://www.actabp.pl/pdf/Supl1_12/s3.pdf

MTMT azonosító: 3240354

IF: 1.185 
Connective Tissue Disorders

P3.6 Oral presentation

\title{
Muscle dystrophy is triggered by type IV collagen alleles affecting integrin binding sites directly or indirectly in Drosophila
}

\author{
Márton Kiss ${ }^{1}$, Ildikó Kelemen-Valkony ${ }^{1}$, András Kiss $^{1}$ Brigitta Kiss $^{2}$, Katalin Csiszár ${ }^{3}$, \\ Mátyás Mink ${ }^{1}$
}

1 University of Szeged, Department of Genetics, Szeged, Hungary

2 Biological Research Centre, Institute of Genetics, Szeged, Hungary

3 University of Hawaii, John A. Burns School of Medicine, Honolulu, HI, USA e-mail:

Matyas Mink<mink@bio.u-szeged.hu>

Congenital muscular dystrophy (CMD), a clinically and genetically heterogeneous group of inherited muscle disorders, is characterized by progressive muscle weakness, fiber size variability, fibrosis, necrotic fibers seen in clusters or grouped necrosis, and by central myonuclei observed normally in regenerating muscle. Regeneration may fail to keep up with ongoing necrosis and muscle fibers are gradually replaced by adipose and fibrous connective tissue, followed by atrophy. Causative mutations in multiple genes have been identified, including the myofibrillar basement membrane components laminin alpha-2, integrin alpha-7 and collagen type VI. Type IV collagen mutations in humans and mammals may manifest with muscle cramps, weakness and dystrophy, but the underlying pathomechanisms remain unknown. In this study we performed immunohistochemistry experiments, mutational and myofibrillar protein analyses and demonstrated that some members of the Drosophila col4a1 mutant series show the onset of CMD. Morphologic alterations of the myofibers meet the requirements of muscular dystrophy. Allele-specific hydrolysis of myofibrillar proteins was observed in these mutants. Myosin heavy chain and tropomyosin were degraded, muscle myosin was devoid of the N-terminal methionine and actin was cleaved by furin. In the proteolytically active col4al alleles glycine substitution affected integrin binding site of the COL4A1 protein, whereas a downstream glycine substitution disrupted the integrin binding site of the opposite COL4A2 chain in the triple helix, judged by uneven integrin expression. Myofibrillar proteolysis was not recorded in those mutants that carried different glycine substitutions. These results collectively demonstrate the broad phenotypical spectrum and pleiotropy of type IV collagen mutations. Proteolytic cleavage of the myofibrillar proteins by furin seems to be a new, yet unknown phenomenon associated with type IV collagen mutations and muscle dystrophy.

\section{References}

Kelemen-Valkony et al. (2012) Matrix Biology 31: 29-37. Kelemen-Valkony et al. (2012) Inherited Myopathies. In: Myopathies: New Research. eds: Howard S. Washington, Chris E. Castillo Jimenez, Nova Publishers, 2012, ISBN: 978-1-62257-372-1.

XXIIIed FECTS and ISMB Joint Meeting, August 25th-29th, 2012, Katowice, Poland 